

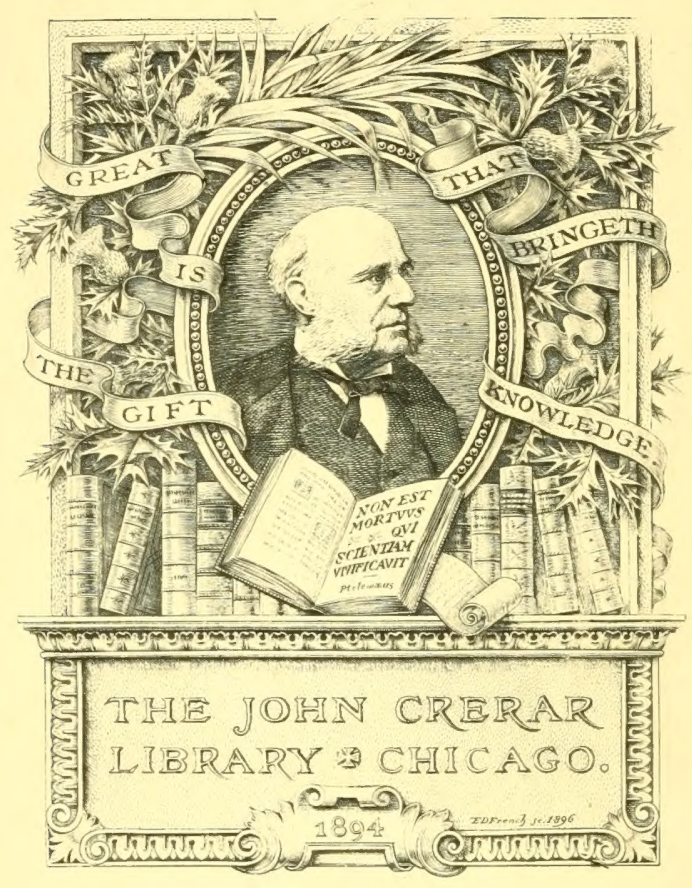






\section{Catalogus systematicus}

\section{DIPTERORUM EUROPAE}

auctore

羁. J. Schiner, Dr。

VINDOBONAE. 


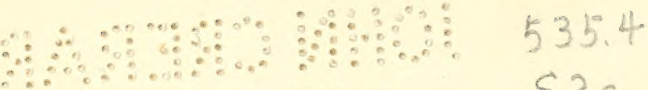

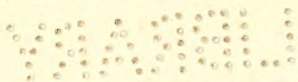

S $3 \mathrm{C}$ 
Seiner Hochwohlgeboren

Herrn Senator

\section{Carl Heinrich Georg von Heyden}

in Frankfurt am Main

hochachtungsvollst gewidmet 
E. old 


\section{Vorrede.}

Es wird Niemand in Abrede stellen, dass ein Catalogus dipterorum Europae einem wahrhaften Bedürfnisse abzuhelfen geeignet sei. Ob dieser mein Catalogus diesem Bedürfnisse wirklich abhelfen werde, weiss ich nicht, hoffe es aber, da derselbe zur Aufstellung und Instandhaltung meiner eigenen Sammlung mir vortreffliche Dienste leistete und leistet. - Was ihm, abgesehen von allem Andern, auch einen wissenschaftlichen Werth geben dürfte, ist das neue System, mit welchem derselbe debütirt. Dieses System beruht auf Brauer's natürlichen Hauptgruppen der Diptera orthorhapha u. cyclorhapha. Die ausführlichere Begründung der weiteren Anordnung des Materiales findet sich in den Abhandlungen der k. k. zoologisch-botan. Gesellschaft (Band XIV. S. 201 u. ff.).

Ich hebe hier nur besonders hervor, dass ein Massstab für die Richtigkeit der befolgten Grundsätze vorläufig nur in der Reihung und Aufeinanderfolge der F amilien gesucht werden dürfe, da die Reihenfolge der Gattungen und in diesen der Arten, bei gegenwärtigem Anlasse noch nicht überall mit voller Begründung durchgeführt werden konnte und hierzu mehr Zeit und ein umfassenderes Vorstudium erforderlich gewesen wären, als mir in diesem Augenblick zu Gebote standen. Sollte eine zweite Auflage dieses Cataloges nothwendig werden, so wird in derselben auch, rücksichtlich der Gattungen und Arten eine consequente, wohlbegründete Reihenfolge zu erwarten sein, da mir bis dahin auch die Andeutungen und Rathschläge der verehrten Herren Collegen hofientlich bekannt geworden sein werden. Den Sachverständigen wird 
es kaum entgehen, dass ich mit meinem neuen Systeme wohl natürliche Kreise und Gruppen, keineswegs aber eine auf-und absteigende Stufenleiter anstrebte und nach meinen Ansichten über ein natürliches System auch nicht anstreben konnte.

Was seit dem Erscheinen meiner Fauna austriaca neu publicirt wurde, ist im vorliegendem Cataloge grösstentheils berücksichtiget worden. Eine Ausnahme macht Robineau-Desvoidy's posthumes Werk über die „Myodaires des environs de Paris“ und der 4. u. 5. Theil von Ronda ni's "Prodromus dipterologiae italicae“. Beide Werke liegen mir vor, es erheischen dieselben aber ein sehr eindringliches und gründliches Studium, um zu einem glücklichen Resultate über die Synonymie der Musciden, über welche sie handeln, zu gelangen. Ich behalte mir desshalb vor, diese beiden Schriften erst bei einer eventuellen nächsten Auflage meines Cataloges zu berücksichtigen.

Möge auch diese meine Arbeit eine freundliche Aufnahme finden und möge sie ein Zeugniss dafür sein, dass es mir ernstlich darum zu thun ist, das Studium der Dipterologie zu fördern und zu erleichtern und dass es mir mindestens nicht an gutem Willen fehlt, meinen verehrten Herren Collegen nach besten Kräften nützlich zu sein.

Wien im Mai 1864.

\section{J. R. Schiner.}




\section{Index.}

Acanthomyia.Schin. . 25|Anthrax. Scop. . . . 27/Bibio. Geoft. . . . . 11 Achalcus. Lw. . . . Acidia. R.-Desv.

42 Antocha. O.-Sak. . 20 Bibionidae . . 10

Aciura. R.-Dess. . . 63 Aphrosylus. Wlk. . . 42 Blepharicera. Mcq. . 23

Acnemia. Wtz. . . . 8 Apodacra. Mcq. . 89 Boletina. Staeg. . . 8

Acnephalum. Mcq. . 33 Apogon. Perr. . . 32 Boletophila. Mg. . . 9

Acrocera. Mg. . . . 30 Arctophila. Schin. . 107 Bombylidae. . . 27

Acroceridae. . . 30 Ardoptera. Meq. . 40 Bombylius. . . 28

Acrometopia. Schin. 72 Argyra. Meq. . . 4.7 Borborinae. . . . 49

Actina. Mg. . . . 28 Argyromoeba. Schin. 28 Borborus. Mg. . . . 50

Actora. Mg. . . . 53 Aricia. R.-Desr. . 80 Brachycampta. Wtz. 7

Aëdes. MIg. . . . 17 Ascia. Mg. . . . . 103 Brachyopa. Mg. . . 107

Agculocera. Meq. . . 86) Asilidae . . . 32 Brachypalpus. Mcq. . 109

Agromyza. Fll. . . . 70 Asilinae. . . . 34 Brachypeza. Wtz. 7

Agromyzinae. ․ 69 Asilus. L. . . . 34 Brachystoma. Mg. . 36

Alliocera. Saund. . . 2 't Asindulum. Ltr. . 9 Braueria. Schin. . 89

Allodia. Wtz. . . . 7 Aspistes. Mg. . . . 11 Braula. Ntzsch. . . . 114

Alophora. R.-Desv. . 101 Asphondylia. Lw. . 3 Braulinae . . . 114

Amalopis. Hal. . . 19 Asteia. Mg. . . . 55 Cacoxenus. Lw. . . 71

Amictus. W. . . 30 Astomella. Ltr. . . 30 Caenia. R.-Desv. . " 38

Anaclinia. Wtz. . . S Asynapta. Lw. . . 4 Callicera. Pz. . . 112

Ananta. Mg. . . 101 Atherigona. Rond. 74 Calliphora. R.-Desv. 81

Anarete. Hal. . . . 10 Atherix. $\mathbf{M g} . . .36$ Callomyia. Mg. . . 102

Anarolius. Lw. . . 33 Athyroglossa. Lw. . 57 Calobata. Mg. ․ . 62

Anatella. Wtz. . . 7 Atissa. Hal. . . . 57 Calobataemyia. Mcq. 85

Andrenosoma. Rond. 34. Aulacigaster. Meq. . 56 Camarota. Mg. . . 59

Anepsius. Lw. . . 4T Axysta. Hal. . . . 58 Campsienenus. W1k. 46

Anisomera.Mg.. . . 18 Azana. Wlk. . . . 5 Campylomyza. Mg. . 4

Anomoia. Wlk. . . 6.3 Bacha. F. . . . 103 Canace. Hal. . . . 58

Anopheles. Mg. . . . 17 Baumhaueria. Mg. . 91 Carphotricha. Iw. . 66

Anthalia. Ztt. . . 10 Berinae. . . . 25 Catocha. Hal. . . \&

Anthomyia. Mg. . . 73 Beris. Ltr。 . . . 25 Cecidomyia. Mg. . . 1

Anthomyinae. . . T3 Besseria. R.-Desr. . 1000 Cecidomyida . 1 
Cocistomuinae.

Cenchridobia. Schin.

Cephalia. Hg.

Cephalomyia. Ltr.

Cephenomyia. Istr.

Ceratitis. il.-Leag.

Ceratonyza. Schin,

Ceratopogon. MIg.

Ceria. F.

Cerinae.

Ceroplatus. Bose.

Ceroxys. Meq.

Chalarus. TVlk.

Chalcochiton. Lw.

Cheilosia. Mg.

Chersodromia. Wlk.

Chironomidae.

Chironomus. Mg:

Chloria. Schin.

Chloropinae.

Chlorops. $\mathbf{M g}$.

Chrysogaster. Mg:

Chrysomyia. Meq.

Chrysopila. Mcq.

Chrysops. Mg. .

Chrysotimus.Lw.

Chrmsitorimue.

Chrysotoxum, MIg.

Chrysotus. Mg.

Chyliza. Fll.

Cinochira. Ztt.

Cistogaster. Ltr.

Clairvillia. R.-Desv. 100 Dexia. Mg. . .

Clasiopa. Stenl.

Cleigastra. Meq.

Clinocera. Mg.

Clinorhyncha. Lw. .

Clista. Mg.. .

Clitellaria. Mg.

Clumio. Hal.

Clusia. Hal.

Clytia. R,-Desf.

Cnemacantha. Mcq. .

Cnephalia. Rond.

Coclopa. $\mathrm{Mg}$. .

Coelosia. IVtz.

Coenomyia. Ltr.

Coenomyidae.

Coenosia. $\mathrm{Mg}^{\circ}$.

Colpodia. IVtz.

Conicera. Mg.

Conopida e.

Conopinae.

20 Cyrtus. Ltr.

27 Dacus. $I I g:$

47 Dasypogoninae.

61 Dasyptera. Schin.

81 Degeeria. MIg.

100 Demoticus. Mcq.

57 Dexinae.

40 Dialyta. Mg.

1 Diamesa. Mg.

86 Diaphorinae. .

24 Diaphorus. $M \mathrm{~g}$.

13 Diastata. Mg.

72 Dichaeta. Mg.

88 Dicranoptycha.

67 Dicranota. Ztt.

98 Didea. Meq. .

50 Dilophus. M̂́g.

1 Dinera. Mcq.

פ5 Dioctria. Mg.

20 Diomyza. Steph.

73 Diplosis. Lw.

4 Dischistus, Lw.

48 Discomyza. $\mathrm{Mg}$.

119 Ditomyia. Wtz.

11:3 1)ixa. IIg.
1 Conops. L.

19) Cordyla. $M \mathrm{~g}$.

63 Cordylura. Ell.

102 Cordyluminac.

102 Corethra. $11 \mathrm{~g}$.

63 Cormoptera. Schin.

71 Corvnocera. Ztt.

13 Corymoneura. Wtz.

112 Corynoscelis. Boh.

112 Criorhina. Meq.

10 Crymobia. Lw.

69 Ctenophora. Mg.

203 Culex. L.

28 Culicidae

105 Cuphocera. Meq.

42 Curtonotum. Meq.

11 Cylindrotoma. Mcq.

12 Cyllenia. Ltr.

68 Cynomyia. R.-Desv.

„9 Cyrtowa. Mg.

59 Cyrtoneura. Meq.
113 Dolichopeza. Curt.

3 Dolichopidae.

73 Dolichopinae.

72 Dolichopus. Ltr.

17 Doros. Mg". . .

53 Dorycera. Mg.

23 Doricerince

11 Drapetis. MIg.

11 Drosophila. Ell.

109 Drosophilinae.

51 Drymcia. Mg.

22. Dryomyza. Fll.

17 Dryomyzinae.

17 Dynatosoma. 11 tz.

99 Echinomyia. Dum.

53 Eclimus. Lw.

21 Ectinocera. Ztt.

29 Eggeria. Schin.

83 Elachiptera. Mec.

37 Elaphropeza. Mg.

81 Elgira. Mg.

33 Elliptera. Schin.

30 Empalia. Wtz.

30 Empheria. Wtz.

36 Dactylolabis. O--Sak.

110 Dasyphora. R.-Desv.

112 Dasypogon. Mg.

72 Diadocidia. Ruthe
81 Eniconeura. Meq. . . 30

32. Ensina. R.-Desv. . $6 . ;$

3\% Ephelia. Schin. . . 19

18 Ephippium. Ltro . . 2'

87 Ephydra. Fll. . . . :8

99 Ephydrinae. . . . . ;6

84 Ephygrobia. Schin. . $: 6$

84 Epicampocera. Mcq. . 96

8. Epicypta. Wtz.... . (;

74 Epidapus. Hal, . .

15 Epidosis. Lm. . . 4

47 Epiphragma. O.-Sak. 19

48 Erioptera. Mg. . . 18

๖ั Eriozona Fürst. . . 106

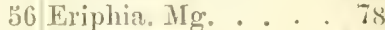

107

$10 \mathrm{~T}$

110

(i;3

;9)

if

31

(i)

\section{1}

2

is

$0: 3$

59

1

(i)

;i)

9

3

i. 3

;i

!)

0

11

1
is 8 8

ij

i

s'

.

;

.

8

(

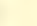

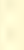

8

3


trivaldskyia. Schin. Frontina. Mg.

Fucellia.R.-Desr.

Gaedia. Mlg.

Gampsocer. Schin. Ot Hon o

Gastrophilus. Leach. 101 Homalura. Mg. .

Geomyza. Fll. . . ¿3̈ Hormomyia. Lw.

Geomyzince. . . อ̈ Hormopeza. Ztt.

Geranomyia. Hal.

Germaria. R,-Dest.

Geroll. $\mathbf{M g}$.

Gitolla. ilg.

Glaphyroptera. WVtz.

Glenanthe. Hal.

Gloma. Mg:

flossigona. Rond. 113 Hydrophorus. Whlb.

Gnophomyia, O.-Sak, 18 Hydrotaea. R.-Desv.

Gnoriste. Mlg.

Gonia. $1 \mathrm{gg}$.

Gonomyia. 0-Sak. 18 Hypenidium. Lw. .

Graphomyia. R.-Desv. 82 Hyperecteina. Schin.

(rymitocheta.R.-B)est.

Gymnomus. Lw.

Gymmopeza. Ztt.

Gymnophora. Heq.

Gymmopternus. Lw.

Gymmosoma. IIg:

Gymosominue.

Gymmostylina. Mcq. .

Habropogon. Lw.

Haematopota. Hg.

Halidaya. Egg.

Halmopota. Hal.

I Haltericerus. Kond.

Hecamede, Hal.

Helomyza. Fll.

Helomyzinae.

Helophilus.Mg: .

Hemerodromia. Mg:

ITemerodrominae.

Hemilea. Lw.

Herina. R.-Desr.

Heteromyza. Eil.

Heteroneura. Fill.

Heteroneurinae.

Heteropeza, $1 V^{\top}$ z.

Heteropogon. Lw.

Heteropterina. $\mathrm{Icq}$

Hexatoma. Mg.

IIilara. Mg:

Hilarella. Rond.

Hilarimorplı, Schin.

Hippobosca. I. .

Hippoboscidae,
98 Hylos. MIg.

30 Hybotinae.

o6 Hydrellia, R.-Desr.

8 Hydrobaenus. Fries.

¿7 Hydromyza. Fll.

39 Hydrophorinae. .

8 Hygroceleuthus, Lw.

98 Hylemyia. R. - Desv.

99 Hypoderma. Ltr.

ธ. Hypophyllus. Lw.

100 Hypostena. Mg.

48 Idia. Mg:

45 Idioptera. Mcq.

101 Ilythea. Hal.

100 Isopogon. Lw.

83 Iteaphila. Ztt.

32 Labidigaster. $\mathrm{M}$ g. .

26 Lampromyia. Heq.

89 Laphria. $\mathbf{M g}$.

38! Laphrinae.

4. Laphystia. Lw.

57 Lasiopa. Brullé.

32) Lasiopogon. Lw.

51 Lasiops. Mg. . .

$10 \mathrm{~s}$ Lasioptera. $\mathbf{M g}$.

40 Lasiosoma. $11 \mathrm{tz}$.

40 Lauxania. Ltr. .

63 Leia. IIg.

68 Leiomyza. Mcq.

52 Leptidae.

72 Leptis. F.

72 Leptogaster. Mg.

1 Leptoyastinae.

33 Leptomorphus. Curt.

90 Leptomyza. Meq. .

26 Leptopa. Ztt.

39 Leptopeza. Meq. .

90 Leptopteryx. Ztt.

36 Leria. R.-Dest.

114. Leskia. R.-Desr.

11 i l estremia. $M g$. .11:kestieminue. . . i

27 Leucopis. Mg. . . . 71

40 Leucostoma. $\mathbf{M g}$. . 86

33 Leucozona. Schin. . 106

74 Liancalus. Lw. . . 46

59 Limnia. R.-Desv. . 5゙́

3 Limnobia. Hg. . . 20

39) Limmobinae . . . 18

58 Limnophila. Acq. . . 19

36 Limnophora. R.-Desv. 78

36 Limosina. Mcq. . . 49

57 Lipara. Mg. . . . . 61

12 Lipoptena. Nitzsch. . 114

72 Lispe. Ltr. . . . 74:

46 Lissa. Mg*. . . . 62

f6 Lobioptera. Whlbg. . 71

79 Loewia. Egg. . . . 87

45 Lomatia. Mg. . . . 28

78 Lonchaea. Hll. . . 67

64 Louchoptera. Hg. . . 115

87 Lonchopterida e. 115

102 Lophosia. Mg. . . 100

14 Loxocera. Mg. . . 61

86 Lucilia. R.-Desv. . 81

83 Lucina. Mg. . . . 53

19. Machaerium. Hal. . 42

58 Macquartia. R.-Desv. 87

33 Macrocera. Pz. . . 9

39 Macronychia. Rond. 90

98 Macropeza. Mg. . . 28

30 Madiza. Fll. . . . 62

33 Mallota. Mg. . . . 108

33 Masicera. HIeq. . . 92

33 Medeterus. Fisch. . . 47

24 Medoria. R.-Desv。 . 85

33 Meghyperus. Lw. . 36

79 Meigenia. R.-Desv. . 94

1 Melania, M $[\mathrm{g} . . .85$

8 Melanophora. MIg. . 85

67 Melanostoma. Schin. 10د

7 Melia. R.-Desr. . . 88

i1 Melithreptus. Lw. . 104

35 Melophagus. Ltr. . 114

36 Merodon. Ng. . . 108

32 Meromyza. Mg. . . 59

32 Mesembrina. Mg. . 83

8 Metopia. Mg. . . 90

j. Micra. Ztt. . . . 100

72/Nicrodon. Ng. . . 112

it) Microdominae. . . 112

114 Micropalpus. Mleq. . 99

51 Micropeza. Mig. . . 61

89 Microphorus. Meq. . 39

\&Microphthalma. Meq. Sí 
Microsoma. Meq. .

Midas. WV.

Midasidae.

Milesia. Ltr.

Milesinae.

Milichia. Mg.

Mitichinae.

Miltogramma. Mg.

Mintho. R.-Desv. .

Mochlonyx. Lw.

Morinin. R.-Desv.

Mosiltus. Ltr.

Mulio. Ltr.

Husca. L.

Muscidate.

Mricidae acalypterae.

Muscidae calypterae.

Muscinae.

Mycetaulus. Lw.

Mycetobia. Mg.

Mycetophila. IIg.

Mycetophilidae.

Mucetophilinae.

Mycothera. Wtz.

Myemis. R.-Desv.

Myobia. R.-Desv.

Myodina. R.-Desp.

Myolepta. Newm.

Myopa. $\mathrm{k}$.

Myopina. R.-Desv.

Nyopinae.

Myopites. Breb.

Myospila. Rond.

Nemestrinn. Itr.

Nemestrinidae.

Nemopoda. R,-Desv.

Nemoraea. R.-Desy.

Nemorius. Rond.

Nemotelus, Geofl'r.

Nephrocerus. Ztt.

Nephrotoma. Mg.

Neurigona. Rond.

Norellia. R.-Desw.

Notiphila. Fll.

Nycteribia. Ltr.

Nycteribidae.

Nyctia. R.-Desr.

Ocemyia. R,-Desv.

Ochthera, Ltr.

Ochthiphila. Fll.

Ochtiphilinae.

Oeydromia.

Ocydrominae.

Ocyptera. Itr.
86 Ocypterinae. . . 1001 Peplomyza. Ha1. 66

31 Odontomyia. Mg.. . 94 Pericoma. Curtis. . 17

31 Oecothea. Hal. . . 52. Periscelis. Lw.

乌3

109 Oedalea. Mg. .

39 Petagnia. Rond.

87

I09 Oedaspis. Lw.

64. Petema. Mg. . . . 98

i) Oedeparea. Lw.

51 Phaeomyia. Schin. . 54

7i Oestridae.

101 Phalacrocera. Schin.

8! Oestromyia. Braner. 102 Phania. $M \mathrm{~g}$.

$8:$ Oestrus. L.

102 Phaninae.

17 Ogcodes. Ltr.

30 Pharynomyia Schin $10^{2}$

85 Olfersia. WV. . . . 114 Phasia. Ltr.

101

61 Oligodranes. Lww.

30 Phasinae

101

28 Oligopogon. Lw.

33 Philygria. Stenh. . 57

82 Olivieria. R.-Desv.

49 Onesia. R,-Desv.

49 Opetia. Mg.

99 Plilebotomus. Rond. .

8:3 Phora. Ltr.

17

103 Phoridae.

48

73 Ojhyra. R.-Desv.

810 pomyza. Fll.

6. Opsebius. Costa.

() Orellia. R.-Desv.

¿ Oreogeton. Schin.

1. Ornithomyia. Itr.

; Orphnephila. Hal.

(i Orthochile. Ltr.

is Orthonema. Mcor

sy Orygma. Mg. .

fis Ortalinae.

$110 \mid$ Ortalis. Fll.

113 Oscinis. Itr.

74 Otites. Istr.

11". Oxycera. Mg.

6:3 Oxyphora. R.-Desv.

81 Oxypterum. Lench.

27 Pachycerina. Meq.

27 Pachyoaster. Mg.

62 Pachuriastrinae.

96 Pachymeria. Steph.

27 Pachyneura. Ztt. .

23 Pachynewinae.

103 Pachyrhina. Meq.

21 Pachystomus. Ltr.

43 Paclyystylum. Meq.

73 Palloptera. Fll. .

56 Pangonia. Ltr.

114. Panyoninae.

114.Paragus. Ltr.

85 Paragusia. Schin. .

113 Parydra. Stenh.

58 iPedicia. Ltr.

72 P'elecocera $\mathrm{Mg}$.

71 Pelina. Hal.

79 Pholocera. R.-Desv. 91

3b Phorostoma, R.-Desv. 84

30 Phortica. Schin. . . 56

64)Phronia. Wtz. . . 7

39 Phthinia. Wtz. . . 8

114 Phthiria. Nlo. . 30

23 Phycodroma. Stenh. כّ

46 Phucodrominae. . 51

110 Phyllodromia. Ztt. . 40

51 Phyllomyza. Hll. . 71

68 Phylloteles. Lw. . 89

69 Physocephala. Schin.114.

60 Phyto. R.-Desv. . . 83

69 Phytomyptera. Rond. 88

24. Phytomyza. Fll. . 69

66 Piophila. Fll. . . . 62

114 Piophitinae. . . . 6\%

67 Pipiza. Fll. . . . 111

23 Pipizella. Rond. . .111

23 Pipunculida e. . . 103

39 Pipunculus. Ltr. . . 103

11 Plagia. Mg. . . . . 98

11 Platycephala. Fll. . 59

21 Platycheirus. St.Farg.105

25 Platycnema. Ztt. . . 103

93 P'latynochaetus. IV. 108

66 Platypalpus. Heq. . 41

27 Platyparea. Lw. . . 63

26 Platypeza. Mg. . .102

111 Platypezidae. . . 102

90 Platystoma. Mg. . . 68

58 Platystominae. . . 68

19 Platystyla. Mg. . . 61

104. Platyura. Mn. . . . 9

58 Plesiastina. WVtz. . 9 9

40 Penthetria. Mg. . 11 Plesina. Mg. . . . 86
39 Penthoptera. Schin. . 18 Plesioneura. ilco. 86
ino Peodes. 1.w. .

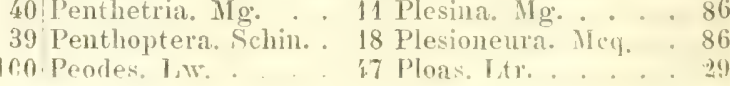

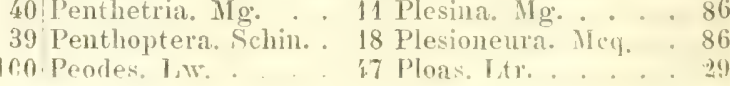


Plocota. St. Farg. . 109! Sapromuzinae. . Poccilostola. Schin. 19 Sarcophaga. Mg. Pogonota. Ztt. Polidea. Mleq. l'ollenia. R.-Desp. Polylepta. Wtz. l'olyphonius. I.w. Porphyrops. Mg. . Promachus. Lw. Prosena. St. Farg. Prosopomyia. Lw. P'sairoptera. IVhlog. Psarus. Itr. Psila. IIg. Psilinae. Psiloconopa. Z $Z$ tt. P'silopus. $11 \mathrm{~g}$. Psilosoma. Ztt. Psilota. Mg. Psychoda. Ltr. l's yehodidae. Pterodontia. Grift. Pterospilus. Rond. Ptiolina. Staeg. Ptychoptera. Mg. . Piychoplerinae. Pycnomala. Gerst. Pycnopogon, Isw. Pyrellia, R,-Desr. Pyrgota. TV. Pyrophaena. Schin. Ragas. TVIk. Redtenbacheria.Schin. Rhacochlaena. Lw. Rhamphidia. Mg. Rhamphina. Meq. . Rhamphomyia. Mg. lihaphinae.

Rhaphium. Mg*.

Rhicuoessa. Lw. Rhingia. Scop. . Rhinophora. R.-Desv. Rhiphidia. Mg: . Rhynchaea. Ztt. Rhynchocephalus. Esch.27 Spilomyia. $\mathbf{M g}$. Rhynchomyia.R.-Desv.83 Spodius. Lw.

Rhypholophus. Kol. . 18 Stegana. Mg. Rhyphidae. Rhyphus. Ltr. Rivellia. R,-Desr. . Thoesclia. R,-Desv. Rymosia. Wiz. Saltella. R.-Desv. sapromyza. Fll.
73 Sitroplire inae.

88 Sarcophila. Rond.

8LSarainae.

8 Sargus. E.

3' Saropogon. Lw.

42 Scatelia. R.-Desv.

3 i. Scatophaga. Mg.

$8 \ddot{3}$ Scatophayinae.

$5: 3$ Scatopse, Geoffr.

68 Scatopsinae.

112 ricenopinialae.

61 Scenopinus. Ltr.

61 Sceptonia. Wtz.

18 Schineria. Rond.

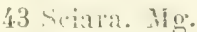

61 Sciarinae.

111 Sciodromia. Hal.

17 Sciomyza. Fll.

17 Sciomvinae.

30 Sciophila. Mg:

36 Scopolia. R.-Desv.

36 Scyphella. R.-Desr.

21 Selachops. Whlbg.

21 Sepedon. Ltr.

24 Sepsinae.

33 Sepsis. Fll.

81 Sericomyia. Mg.

09 Sericomyinae.

103 Sicus. Scop.

39 Simulia. Ltr.

$89 \mathrm{~S}$ imulidae.

65 Siphona. Mg.

20 Siphonella. MIey.

19 Spania. Mg.

37 Spaniocera. IVtz.

4.2 Spatigaster. Rond.

4.2 Sphaerocera. Ltr. .

31'Sphacrogaster. Ztt.

107 Sphecomyia. Ltr.

86 Sphegina. Mg:

20 Spilogaster. Hrcq.

62 Spilographa. Lw. .

23 Stenopogon. Lw.

23 Stenopteryx. Leach. 114 Tinia. IV.

68 Stichopogon. IJw. . . 33 Tipula. L.

89 Stomoxys. Geoffr.

7 Stratiomydae.

6.2. Stratiomynae.

(if Stratiomys. Feollir.
66 Subula. Hg. . . . 20̈

83 Sybistroma. $\mathrm{Mg}$. . . .

83 Sycorax. Curtis. . . 17

84 Syllegoptera. Rond. 73

25 Sylvius. Mg... . . 26

23 Symplecta. $11 \mathrm{~g} . \quad . \quad 18$

32 Sympyenus. Lw. . . 17

58 Syntemna. Wtz. . 8

51 Syntomocera. Schin. 8.t

šl Syntomogaster. Schin.101

10 Syntolmon. Lw. . 43

10 Syritta. St. Earg. . 109

30 Syrphidae. . . 103

30 Syrphinae. . . . 103

¿̈ Syrphus. F. . . 104

99 Systenus. Lw. . . 43

4ystoechus. Lw. . 。 9

4 Tabanidae. . . 26

4 Tabaninae. . . 26

33 Tabanus, L. . . . 26

53 Tachina. Mg. . . . 9\%

8 Tachininae. . . . 85

86 Tachydromia. Mg. . 42

5̋ Tachydrominae. . . \$1

59 Tachytrechus. Stann. $40 ̈$

s. Tanypeza. Fll. . . 61

62 Tanypezinae. . . . 61

63 Tanypus. Mg. . . I'

107 Teichomyza. Heq. . 59

107 Tephritis. Ltr. . . 68

113 Tetanocera. Fll. . 54

10 Tetanocerinae. . . 54

10 Tetanops. Fll. . . 69

88 Tetamura. Ell. . 61

60) Tetragoneura. IVtz. 8

36. Teuchophorus. Lw. . 17

1 Thelaira. R.-Desv. . 8:;

105 Thelida. R.-Desv. . 32

50 Themira. R.-Desv. . 62

30 Thereva. Itr. . . 31

112 Thereridae. . . 31

103 Theria. R.-Desv.. 8t

80 Therina. $\mathrm{Mg}$. . . . 80

64 Thinophilus, Lw. . 47

109 Thrypticus. Gerst. . RT

11 Thryptocera. $11 \mathrm{c}$. 88

5. Thyreophora. $11 \mathrm{~g}$. .

33 Thyreophorinae. . 51

83 Tipulidae. . . 18

23 Tipulinae. . . . 21

23 Toxophora. Mg. . . 30

2. 'Trichocera. $M g$. . 19 
Irichomyia. Curtis. I'i|Trypetinae. . . 633 Xanthogrammoschin 10h Trichonta. Wtz. . . 7 Tryphera. Mg. . . 88 Xestomyza. Mg. . . 3 ! Trichopeza. Rond. . 40 Ula. Hal. . . . 19 Xiphocerus. Meq. . 32 Trichosticha. Schin. 18 Ulidia. Mg..... . 68 X ylophagida e. . 25 Triclis. 1,w. . . . 33 Ulidinae. . . . 68 Xylophagus. Mg. . 25 Tricyphona. Ztt. . . 19 Ulomyia. Wlk. . . 17 Xylota. Mg. . . . 109 'l'riglyphus. Lw. . . 111 Uromyia. Mg. . . 100 Xysta. Mg. . . . 101 Irigonometopus. Mcq. 69 Urophora. R-Desq. 64 Zeuxia. Mg. . . . 84. Trimerina. Heq. . . \$6 Usia. Ltr. . . 30 Zodion. Ltr. . . 113 Trineur. Mg. . . . 48 Vermileo. Heq. . . 35 Zophomyia. Meq. . 99 Triogma. Schin. . . 21 Volucella. Geoftr. . . 107 / ygomyia. Wtz. . . ¿ Trixa. Mg. . . . .98 Volucellinae. . . .107, Zygomeura. Mg. . . . Tropidia. Mg. . . $\$ 09$ Winertzia. Schin. . 90 Trypeta. Mg. . . 64. Xanthochlorus. Iw. . 43 


\section{Diptera orthorhapha.}

\section{A. Nematocera.}

\section{a. Oligoneura.}

I. Cecidomyldae.

Cecidomyinae.

1. Heteropeza. Wtz. pygmaea. Wtz. nerrosa. W'tz.

2. Diomyza. Steph. Lasioptera. $\mathrm{Mg}$. pusilla. $\mathbf{M g}$. stygia. Mg. obfuscata. Mg. fuliginosa. Steph.

3. Lasioptera. Mg. Tipula. I. arundinis. Schin. auricincta. Wtz. eryngii. Giraud. picta. $\mathrm{Mg}$. flexuosa. Wtz. rubi. Schrk. argyrosticta. $\mathrm{Mg}$. pulchra. Mig. obtusa. I,w. albipennis. $\mathrm{Mg}$. fusca. Mg: minuta. 7t.t. argentata. Lw。 annulata. Costa. berberina. Schrk.

4. Clinorlyyeha. Lw. Ozirhyncus. Rond. chrysanthemi. Lw. crassipes. Wtz. longicollis. Rond.

:. Spaniocera. Wtz. Brachyneura. Rond. squamigera. Wtz. fuscogrisea. Rond.

6. Cecidom y ia. Mg. Tipula.Auct. - Oligotroplues Ltr. - - Dasyneura. Rond.

rosaria. I Iw. salicis. Bremi. cinerarum. Hardy. salicis. Sehrk. salicina. Bouché. Degeeri. Bremi. gallarum salicis. $\mathrm{Hrd}$. albipennis. Wtz. circinans. Giraud. saliciperda. L. Duf. terebrans. Liw. inclusa. Ffld. scutellata. Boié. Giraudii. Ffld. albilabris. Wtz. riparia. Wtz. persicariae. L. euphorbiae. Bouché. subpatula. Bremi. capitigena. Bremi. salicina. Deg. Frischii. Bremi. heterobia. Lw. iteophila. Lw. terminalis. Lw. saligna. Hardy. marginemtorquens. $\mathrm{Br}$. cardaminis. Wtz. circumdata. Wtz. crataegi. Wtz. papareris. Wtz. sisymbrii. Schrk. brassicae. IVtz. acrophila. IVtz. strobi. Wtz. betulae. Wtz. galii. Lw. pratorum. IVtz. veronicae. Bremi. galeobdolontis. Wtz. serotina. Wtz. urticae, Perris. ulmariae. Bremi. bursaria. Bremi. pyri. Bouché. 
saliceti. Lw. parida. IVtz.

cerris. Kollar.

cerrifoliae. Passerini.

Klugii. Mg.

artemisiae. Bouché.

tubifex. Bonché.

foliorum. Lw.

lithospermi. Lw.

plicatrix. Lw.

millefolii, Lw.

syngenesiae. $1 \mathrm{w}$.

bryoniae. Bouché.

acerina. Giraud.

secalina. Lw.

destructor. Say.

poae. Bosc.

hyperici. Bremi.

juniperina. Géné.

obscura. Rond.

luteofusca. Rond.

innotata. Wlk.

costalis. Wik.

sociata. Wlk.

decolorata. TVlk.

tecta. WVIk.

basalis. WVIk.

cinctipes. WVlk.

testacea. WIk.

discolor. Wlk.

strobilina. Bremi.

pennicornis. L.

longicornis. L.

palustris. L.

albipalpis. Mg.

carnea, $\mathrm{Mg}$.

bicolor. $\mathrm{Mg}$.

fuscipennis. Mg.

griseicollis. Mg.

griseola. Mg.

lateralis. $\mathrm{Mg}$.

nerrosa. Mg. nigra. MIg.

nigricollis. Mg.

nigripemis. $\mathrm{Mg}$.

obscura. Mg.

pallida. $\mathrm{Mg}$.

pictipennis. Mg.

pratorum. Mg.

scutellata. $\mathbf{M g}$.

tristis. MIg.

variegata. Mg.

varia. Mcq.

vittata. $\mathrm{Mg}$.

Westermanni. Mg.

formosa. Bremi.

grisea. Bremi.

bicolor. Bremi.

raricolor. Bremi.

fenestralis. Bremi.

limbitorquens. Bremi.

stachydis, Bremi.

onobrychidis. Bremi.

gibba. Ztt.

hirticoluis. 7tt.

laeta. Ztt.

monilicornis. Ztt.

nigricornis. 'Ztt.

nig'ritarsis. Ztt.

niveitarsis, Ztt.

nudipennis. Ztt.

pallidula. Ztt.

pictiventris. Ztt.

sordida. Ztt.

tarseolata. Ztt.

testacea. Ztt.

vulnerata. Ztt.

Wimnertzii. Ztt.

aurantiaca. Mcq.

pygmaea. Mcq.

lutea. Ltr.

ericae - scopariae.

L. Duf:

populi. L. Duf。

viminalis. Westw. obscuripes. Dahll.

surora. Mannerh.

entomophila. Perris.

cerealis. Kollar.

7. Diplosis, Lw. Tipula.

Dg. - Cecidomyia.

Auct. - Diplosis,

Bremia u. Contarinia.

Rond.

signata. Wtz.

latibulorum. Wtz.

mutabilis. Wtz.

sylvatica. Wtz.

polypori. Wtz.

minuta. Wtz.

socialis. Wtz.

suliterranea. Ffld.

geniculata. Wtz.

peregrina. Wtz.

nana. Wtz.

tibialis, $1 /$ tz.

fascipennis. IVtz.

pulchella. Wtz.

callida. Wtz.

arcuata. Wtz.

maculata. IVtz.

loti. Deg.

medicaginis. Bremi.

linariae. Wtz.

rumicis. Lw.

ochracea. Wtz.

modesta. Wtz.

xanthopyga. IVtz.

cingulata. WVtz.

dumetorum. Wtz.

Haveolata. Wtz.

agilis. Wtz.

limbata. IVtz.

botularia. IVtz.

? fraxini. Bremi. coniophaga. Wtz.

invocata. Wtz. 
impudica. Wtz. pini. Deg.

laterella. Ztt. pini - maritimae.

L. Duf.

digitata. Wtz.

tremulae. Wtz.

praecox. Wtz.

centralis. Wtz.

? annulipes. $\mathrm{Mg}$.

ceomatis. Wtz.

cerasi. Lw.

cilipes. Ntz.

pisi. Wtz.

decorata. Wtz.

asclepiadis. Giraud.

anthemidis. Lw.

guttata- Lw.

jacobaeae. Lww.

brachyntera.Schwaegr.

inulae. Lw.

dactylidis. Lw.

annulipes. $\mathrm{Mg}$.

Llava. MIg.

hilarella. Ztt.

verna. Curtis.

helianthemi. Hardy.

tritici. Kirby.

paronia. Lw.

tenuis. Lw.

unicolor. Lwv.

simplex. I w.

notabilis. WIk.

dolosa. Wlk.

tendens. Wlk.

conformis. Wlk.

areuata. Wlk.

flexa. Wlk.

linearis. WVIk.

tenuicornis. Wlk.

Leacheana. WIk.

moesta. Wlk.

sobrie. IVlk. articulata. Wlk.

latiuscula. WIk.

apicalis. Wlk.

fixa. Wlk.

marginata. Wrlk.

solemnis. Wlk.

ciliata. WIk.

moesta. WIk. (bis)

pubescens. Wlk.

disjuncta. Wlk.

filipes. Wlk.

evanescens. Wlk.

maura. Wlk.

invaria. WVlk.

repleta. Wik.

atripes. WIk.

semiopaca. IVlk.

expandens. TVlk.

incompleta. Wlk.

terminalis. WVlk.

albipes. WVk.

brericornis. Wlk.

latipennis. Wlk.

elegans. WVlk.

concinna. Wlk.

albiceps. Wlk.

concolor. WIk.

abrupta. Wlk.

decora. WVlk.

plagiata. Wlk.

nubeculosa. Wlk.

brevis. Wlk.

Woeldickii. Contar.

cerealis. Rond.

aphidimyza. Rond.

8. A sphondyli a. Isw.

Tipula. Deg. - Ceci-

domyia. Auct. -

Phytophaga. Roud. verbasei. Vall.

seroploulariae, Schin. echii. Lw.

sarothamni. Wוג. ribesii. Ilg.

genistae. Lw.

coronillae. Rond.

crassicomis. Wlk.

albonotata. WVlk.

aethiops. Wlk.

plena. Wlk.

innotata. Wlk.

fusca. Mg.

9. Horm om y i a. Lw.

Tipula. L. - Ceci-

domyia. Auctor. -

Angelinia. Rond.

fasciata. $\mathrm{Mg}$.

rosea. Ztt.

producta. Mg.

fasciata. Bremi.

cucullata. Mg.

extrema. IV!k.

grandis. Mg:

cornuta. Bremi.

amoena. Lw.

grossa. Bremi.

fagi. Hartig.

gibbosa. Rond.

protuberans. Ztt.

juniperina. L.

flavimaculata. 'Ztt.

piligera. Lw.

annulipes. Hartig.

elegans. WVtz.

bipunctata. IVtz.

floricola. Wtz.

lentipes. Wtz.

capleae. Wtz.

graminicola. IVtz.

fuscicollis. $\mathrm{Mlg}$.

crassipes. Lw.

hirtipes. Ztt.

immunda. Z/tt.

funesta. Wlk.

colni. Giraud.

amplik. W'k. 
10. Colpodia. Wtz. 'orycondyla. Rond. pt. angustipennis. Wtz.

11. Epidosis. Lw. Cecidomyia. Auct. - Epidosis und Dirhyza LW.

defecta. Jww. pallipes. Wtz. albimana. Wtz. albitarsis. $M g$. leucopeza. Mg. dorsalis. IVtz. gracilis. Wtz. renusta. Wtz. analis. Wtz. nodicornis. Wtz. nemoralis. Wtz. valvata. Wtz. corticalis. Lw. fragilis. Lw. longipes. Lw. phragmitis, Giraud. nigrina. WIk. sericata. Lw. lateritia. Lw. rhodophila. Hardy.

12. Asynapta. Lw. Cecidomyia. Auct. Asymapta und Winnertzia. Rond. pectoralis. Wtz. lugubris. IVtz. longicollis. Lw. longicauda. Lw. Bouchéana. Lw. salicis. Bouché. tenella. Whi.

Lestreminae.

13. Canı p y om y za. Mg. Cecidomyia. Mg. ol - Micromyia. Rond. halterata. 7tt. atra. Mg.

bicolor. $\mathrm{Mg}$.

flavipes. Mg:

pallipes. Ztt.

aceris. Mg.

albicornis. Mg.

fuscipes. $\mathbf{M g}$.

Inculenta. Mg.

breripennis. Wlk.

globifera. Whlk.

lucorum. Rond.

14. Catocha. Hal. Macrostyla. Wtz. latipes. Hal.

brevinervis. Ztt. aprilina. Ztt.

15. Lestremia. Mg. Tipula. F. Chironomus. F. - Sciara. Mg. ol. - Mimosciare. Rond. - Lestromia u. Cecilogona. Lw.

lencophaea. Mg. cinerea. Meq. fusca. $\mathrm{Mg}$. carnea. Lw.

\section{II yce tophilldac.} Sciarinae.

16. Epidapus. Hal. yenaticus. Hal.

17. 7ygoneura. Mg. sciarina. Mg. gracilis. WVtz. pumila. $\mathbb{N}^{\top} \mathrm{tz}$. tenella. Lw.

18. Sciara. Mg. - Tipula. L. F. Scop. -Hirtea. T. - Molobves. Ltr. - Planetes. Wik. - Plenetella. ITestw. pt.
Thomae. L.

hemerobioides. Scop.

lateralis. $\mathrm{Mg}$. morio. Ztt. ol. humeralis. Ztt. scutellata. Staeg. bilineata. Streg. analis. Egg. morio. 1 .

forcipata. F. florilega. Mg. nigripes. $\mathrm{Mg}$. flavimana. Ztt. rufiventris. $\mathrm{Mg}$. bicolor. Mg. amulata. Mg. umbratica. Ztt. longiventris. Dahlb. hyalipennis. $\mathbf{M g}$. nitidicollis. $\mathbf{M g}$. fuscipennis. $\mathrm{Mg}$. distincta. Staeg. praecox. $M g$. fuscipes. Mg. fucata. Mg. quinquelineata. Mcq. nervosa. $\mathrm{Mg}$. pulicaria. Mg. sylyatica. Mg. Giraudii. Lgg. pallipes. F. aprilina. $\mathrm{Mg}$. longipes. Mg. allinis. Ztt. flavipes. Pz. trivittata. Staeg. tilicola. $\mathrm{L}, \mathrm{w}$. ruficauda. My. vitripennis. $M g$. fenestrata. Mg. scatopsoides. IIg. minima. $M g$. pusilla. Me: 
brumnipes. Mig. hirticornis, Mg. nemoralis. $\mathrm{Mg}$. lutea. IIg. costata. Mg. carbonaria. Mg. caudata. WVlk. compressa. Wlk. vittata. $\mathrm{Mg}$. sericata. Mg. glabra. Mg. viridipes. Mcq. extrema. Wlk. pilosa. Staeg. strigata. Staeg. pectoralis. Staeg. venosa. Staeg. modesta. Staeg. iridipennis. Ztt. variabilis. Ztt. flavicauda. Ztt. trochanterata. Ztt. picipes. Ztt. sordida. Ztt. sordidella. Ztt. hirtipennis. Ztt. testacea. Ztt. variabilis. $Z$ ttt. brevicornis. Ztt. persicariae. Ztt. vittigera. Ztt. fenestralis. Ztt. vernalis. Zitt. nitidula. Ztt. atomaria. Ztt. rufescens. Ztt. lestacea. Ztt. ol. subterranea. Märkl. ingenua. L. Duf. pruinosa. Bouché. elongata. Bouché, gracilis. Wlk. pallida. Wlk. compressa. WIk. caudata. WVlk. Bigoti. Laboulb. Mycetophilinae. 19. Azana. Wlk. Boletina. Staeg. anomala. Staeg. scatopsoides. Wlk. 20. Acnemia. Wtz. - 22. Dynatosoma. Wtz. Leia. Mg. - Boletina. Staeg. amoena. Wtz. nitidicollis. Mg. longipes. Wtz.

21. Zy gom yia. Wtz. Mycetophila. Auctor. canescens. Wtz. flaviventris. Wtz. nitida. Stann. notata. Stam!. pictipennis. Staeg. binotata. Hal. valida. Wtz. vara. Staeg. oligoneura. Stann. 2.2. Sceptonia. Wtz. Mycetophila. Auctor. concolor. Wtz. costata. v. d. Wlp. nigra. $\mathrm{Mg}$. nitida. Mg.

23. Cordyla. Mg. Pachypalpus. Staeg. crassicornis. Mg: brevicornis, Staeg: valida. Wlk. ritiosa. Wtz. nitens. Wtz. semiflava. Staeg. Haviceps. Staeg. fuscit. Lotr. fasciata. Mg. cinerea. Ztt. fulveola. Hal. atra. Mer. anomala. Mcq. canescens. Ztt. murina. Wtz. crassipalpis. L.Duf. obscuripennis. Wtz. - Mycetophila. Auct. fuscicornis. Mg. praeusta. $\mathrm{Mg}$. nigricoxa. Ztt. 20. Mycetophila. Mg. - Sciara. F. punctata. Mg. striata. F. trivialis. $\mathrm{Mg}$ cunctans. $\mathrm{Mg}$. semicincta. Mg. unicolor. Mg. sufa. Mcq. lineola. $\mathrm{Mg}$. ruficollis. Mg. lurida. $\mathrm{Mg}$. monostigma. Mg. centralis. Mg. unipunctata. $\mathrm{Mg}$. stolida. Wlk. pumila. Wtz. unicolor. Stamn. bimaculata. F. pictula. $\mathrm{Mg}$. arcuata. Ztt. ol. vittipes. Ztt. nebulosa. Stann. xanthopyga. Wtz. fulva. Wtz. flava. IVtz. siguata. Stann. l,unata. E. astigma. Nig. 
lunata. Mg.

? arcuate. Mg. spectabilis. Wtz.

fraterna. Wtz.

marginata. Wtz.

rudis. Wtz.

amoella, $W^{\gamma} \mathrm{t}$.

biusta. Mg:

blanda. W'z.

maculipennis. Wtz.

hamata. Wtz.

gratiosa. Wtz.

modesta. Wtz.

luctuosa. Mg.

tarsata. Wtz.

cingulum. Mg:

morosa. Wtz.

gibba. Wtz.

sordens. IV.

humeralis. TV.

flaviceps. Mg.

arcuata. Mg.

attenuata. $\mathrm{Mg}$.

ruficornis. Mg.

semillava. Mg.

pallipes. $\mathrm{Mg}$.

taeniata. Mg.

alterna. $\mathrm{Mg}$.

festiva. Mg.

pallidicornis. Mcq.

flavipes. Mcq.

pygmaea. Mcq.

nana. Meq.

incompleta. Mcq.

sericea. Mcq.

sericoma. $\mathrm{Mg}$.

lunulata. Meq.

brumnea. Meq.

annulata. Meq.

trimacula. Meq.

maculata. Meq.

picta. Meq. atra. Mer.

lycogalae. Perris.

modesta. I. Duf.

inermis. L. Duf.

punctipennis. Stann.

Anderschii. Stamn.

nigricincta. Stam.

strigata. Staeg.

caudata. Staeg.

abdominalis. Staeg.

discicollis. Staeg.

fuscipennis. Staeg.

minuta. Staeg.

ricina. Staeg.

venosa. Staeg.

tarsata. Staeg.

paludosa. Staeg:

thoracica. Ztt.

lutescens. Ztt.

fuscula. Zitt.

flexuosa. Ztt.

obsoleta. Ztt.

cinerea. Ztt.

rufescens. Ztt.

ferruginea. Ztt.

uninotata. Ztt.

unimaculata. Ztt.

canescens. Ztt.

grisea. Ztt.

nigricollis. Ztt.

atricauda. Ztt.

fuscicauda. Ztt.

melanopyga. Ztt.

griseola. Ztt.

aterrima. Ztt.

parvula. Ztt.

nana. Staeg.

nigricornis. Ztt.

exigua, Ztt.

curona. Ginmerth.

ferruginea. Wrik.

flava. Wlk.

nigritula. WIk. selecta. Wlk.

sobria. WVlk.

conformis. WIk.

finalis. Wlk.

terminalis. WIk.

longicornis. Wlk.

indecisa. IVlk.

leioides. WIK.

26. Mycothera. Wtz. Mycetophila. Auctor. dimidiata. Staeg. semifusca. Mg.

27. Epicypta. IVtz. Mycetophila. Auctor. punctum. Stann. scatophora. Perris. trinotata. Staeg. 28. Exechia. Wtz. Tipula. Deg. - Mycetopliza. Auct.

intersecta. $\mathrm{Mg}$. cingulata. Mg. subulata. Wtz. tenuicornis. v. d. WVlp. pulchella. Wtz. trivittata. Staeg. leptura. Mg. fungorum. Deg. fusca. Ltr. lateralis. $\mathrm{Mg}$. guttiventiis. Mg. seriata. Mg. concinna. Wtz. spinigera. Wtz. serrata. Wtz. contaminata. IVtz. confinis. Wtz. cincta. Wtz. dorsalis. Staeg. ? diagonalis. $11 \mathrm{~g}$. bicincta. Staeg. interrupta. Z/tt. maculipemis. Stanu. 
speciosa. Wtz.

festiva. Wtz.

pallida. Stanu. ochracea. Ztt.

fulvicollis. Stann.

Macquarti. Stann.

longicornis. v. d. $W^{\top} \mathrm{p}_{\mathrm{p}}$.

29. Phronia. Wtz. -

Mycetophila. Auct.

flaricollis. Wtz.

austriaca. Wtz.

ammulata. WVtz.

lepida. IVtz.

apicalis. Wtz.

signata. Wtz.

strenua. Wtz.

triangularis. Wtz.

nitidiventris. r. d. WIp.

forcipata. Wtz.

forcipula. Wtz.

rittata. Wtz.

flavicauda. IVtz.

vitiosa. Wtz.

humeralis. Wtz.

pygisiaca. Wtz.

basalis. Wtz.

laeta. IVtz.

tenuis. W'tz.

cinerascens. $\mathbb{W t z}$.

truncata. Wtz.

rustica. Wtz.

longipes. Wtz.

flavipes. Wtz.

obtusa. Wtz.

crassipes. Wtz.

squalida. Wtz.

pigra. Wtz.

apicalis. $M g$.

dispar. Stamn.

30. Anatella. Wtz.

gibba. Wtz.

ciliata. Wtz.

flavicauda. Wtz.
31. Trichonta. Wtz, Mycetophila. Auct. melanura. Staeg. submaculata. Staeg. trossula. II'tz. simplex. Wtz. funebris. Wtz. umbratica. IVtz.

obesa. Wtz.

vitta. $\mathrm{Mg}$.

gracilis. Stamm.

3\%. Brachycampta. Wtz. - Mycetophila.

Auctor.

altermans. Ztt.

bicolor. Mer.

brachycera. Ztt.

serena. WVtz.

amoella. Wtz.

hastata. Wtz.

caudata. Wtz.

griseicollis. Staeg:

picta. W.

brevicornis. Mg:

flaviventris, $\mathrm{v}$. d. Wlp.

proxima. Staeg.

33. Allodia. Wtz.

Mucetophila. Auctor. obscura. Wtz.

crassicornis. Stam.

spinicoxa. Ztt.

punctipes. Staeg.

ornaticollis. $\mathrm{N} \mathrm{Fg}$.

lugens. TV.

grata. Mg*.

analis. $\mathrm{Mg}$.

barbipes. Wtz.

34. Rymosia. Wtz. -

Mycetophila. Auctor. discoidea. Mg:

fasciata. Mg. maculosa. Ztt. signatipes. v. d. $\mathrm{WV} \mathrm{l}_{\mathrm{p}}$,

spinipes. Wtz.

comnexa. Wtz.

truncata. Wtz.

aftinis. Wtz.

placida. IVtz.

macrura. Wtz.

cristata. Staeg.

gracilis. Wtz.

maculosa. Mg.

fenestralis. Mg.

rufa. Mcq.

domestica. Mg.

Schummelii. Stamm.

3อ. Brachypeza. Wtz.

bisignata. Wtz.

armata. Wtz.

obscura. Wtz.

hilaris. Wrtz.

36. Docosia. Wtz. -

Mycetophila. Auct.-

Leia. WIk.

sciarina. $\mathbf{M g}$.

gilvipes. IVIk.

ralida. Wtz.

37. Coelosia. Wt\%. -

Boletina. Staeg.

Hava. Staeg:

flavicauda. Wtz.

38. Leia. Mg. - Myce-

tophila. Mg. ol. Mcq. elegans. Wtz.

terminalis. Mg.

variegata. Wtz.

ferruginea. . d. $\mathrm{Wl}_{\mathrm{l}}$.

dimidiata. $\mathbf{M}$ g.

lutea. $\mathbf{M g}$.

ammulata. Mcq.

crassicornis. Curtis.

pulchella. Curtis.

octomaculata. Curtis.

pubescens. TIlk. 
fumosa. Wlk. basalis. Wik. infera. IVlk. parallela. Wlk. diversa. Wlk. defecta. Wlk. posticata. Ztt. apicalis. Ztt. brevicornis. Ztt.

helvola. WIk.

39. Glaph y roptera. Wtz. - Leia und Mycetophila. Auct. fascipennis. MIg. flavicornis. Mg. cruciger $\alpha$. Ztt. consobrina. Curtis. unicolor. Wtz. fasciola. Mg. cylindrica. Wtz. subfasciata. Mg. Marklini. Ztt. picta. Mg.

bifasciata. Gimmerth. alternans. Wtz. bilineata. Wtz. Winthemi. Lehm. bimaculata. Mg. borealis. Wtz.

bimaculata. Staeg. stigmatella. F. d. WVlp. 40. Phthinia. Wtz. humilis. IVtz. thoracica. Wtz. gracilis. Wtz.

41. Gnoriste. Mg. apicalis. $\mathrm{Mg}$. bilineata. Ztt. trilineata. Ztt.

42. Boletina. Staeg.- Leia. Mg. trivittata. $M g$. basalis. Mg.

nigia. Ztt.

humeralis. Ztt.

nigricoxa. Staeg.

sciarina. Staeg. obscurella. Ztt.

dispar. IVtz.

dubia. Staeg.

groenlandica. Staeg.

trivittata. Ztt.

basalis. Staeg. analis. $\mathrm{Mg}$.

dubia. Mg.

borealis. $\mathrm{Ztt}$.

brevicornis. Ztt.

consobrina. Ztt.

tenella. Ztt.

unifurcata. Zitt.

plana. IVlk.

43. Anaclinia. Wtz. -

Mycetophila. Mg: -

Leia. Mg.- Boletinu.

Staeg.

memoralis. Mg.

44. L e p t o m or ph h s.

Curtis.

Walkeri. Curtis.

4.․ Syntemna. IVtz. morosa. Wtz.

16. Diadocidia. Ruthe. 31 . Empheria. Wtz. -

- Mycotobia. Mg. Macroneura. Mcq. ferruginosa. $\mathrm{Mg}$. flavicans. Ruthe.

Winthemi. Mcq.

47. 'T e tragon e ura. Wtz. - Sciophila.

Auctor.

hirta. Wtz.

dissimilis. Ztt.

sylvatica. Curtis.

distincta. $\mathrm{Wtz}$. pubescens. Ztt.

melanoceras. Wlk.

48. Empalia. Wtz. -

Sciopliila. $\mathrm{Mg}$.

vitripennis. $\mathbf{M g}$.

49. L asiosoma. Wtz. Sciophila. Auct.

hirta. $\mathbf{M g}$. pilosula. Zit.

nitens. Wtz.

interrupta. WVtz.

pilosa. $\mathrm{Mg}$.

analis. Wtz.

fulva. Mg.

limbatella. Ztt.

thoracica. Staeg.

nigriventris. Meq.

lutea. Meq.

tenuis. Wtz.

rufa. Mg.

cincta. Wtz.

robusta. Wtz.

50. Polylepta. Wtz. -

Sciophila. Mg.

leptogaster. Wtz.

undulata. Wtz.

splendida. TVtz.

collaris. Mg.

striata. $\mathbf{M g}$.

lineola. $\mathbf{M g}$.

proxima. WVtz.

insignis. Wtz.

pictipennis. IIal.

formosa. Wtz.

tarsata. Wtz.

52. Sciophila. Mg. -

Tipula. F.- Platyura. Mg. ol. -. Asindulum, Ltr. pt. sordida. Wtz.

Sciophila. Auct. 
flavicollis. Ztt. inanis. Wtz. alacris. Wtz. pallida. Wtz. maculata. Mg. platyura. $\mathrm{F}$. hyalinata. Mg. annulata. $\mathrm{Mg}$. punctata. Mg. decorosa. Wtz. notabilis. Staeg. limbata. Wtz. fasciata. Ztt. munda. Wtz. cinerascens. Mcq. fuscata. Wtz. incisurata. Ztt. exigua. Wtz. ornata. Mg. tumida. Wtz. lucorum. Wtz. nigricornis. Ztt. fraterna. Wtz. apicalis. Wtz. aftinis. Staeg. fusca. Mg. flava. Wtz. trilineata. Ztt. circumdata. Staeg. fimbriata. Mg. melania. Wtz. lugubris. Wtz. cingulata. $\mathrm{Mg}$. elegans. Ruthe. marginata. Mg. tergemina. Ruthe.

ferruginea. MIg. unimaculata. Meq. ochracea. MIeq. nigra. Mcq. halterata. Staeg. trivittata. Ztt. sororcula. Ztt.

fasciata. Ztt. ol. diluta. Ztt.

vittiventris. Z Ztt. melanogaster. Ztt. griseovittata. Ztt. notata. Ztt. univittata. Ztt. nitida. Ztt. ruficollis. Ztt. guttiventris. Ztt. geniculata. Ztt. zonata. Ztt. fenestella. Curtis. rufilatera. Wlk. tenuis. Wlk. hilaris. WVlk. maura. Wlk. congrua. Wlk. ochracea. Wlk. compressa. Wlk. aliena. Wlk. fasciata. Gimmerth. lineata. Gimmerth.

53. Boletophila. Mg. Macrocern. Mg. ol. Messala. Curtis. fusea. Mg. hybrida. Mg. ol. bimaculata. Ztt. maculipennis. Wlk. Saundersii. Curtis. cinerea. $\mathrm{Mg}$. tenella. Wtz.

ว̆. N a c rocera. Pz. Mg. fasciata. Mg. lutea. Mg. vittata. $\mathrm{Mg}$. pusilla. Mg. nana. IIcq. crassicornis. Wtz. nigricoxa. Wtz. centralis. $\mathrm{Mg}$. maculata. Mg. angulata. Mg. vittata. Meq. stigma. Curtis. alpicola. Wtz. obscura. Wtz. phalerata. Mg. fascipennis. Staeg. maculipennis. MIcq. apicalis. Hoft'm.

5. Plesiastin a.Wtz. Mycetobia. Mg. Staeg. - Ceroplatus. Ztt. - Symmerus. Wlk. ammulata. Mg. flava. Ztt. ferruginea. Wlk. apicalis. Wtz.

36. Ditomyia. Wtz. Mycetobia. Mg. Platyura. Mlcq. fasciata. $\mathrm{Mg}$.

bifasciata. Meq.

trifasciata. Wtz. macroptera. Wtz. 57. Mycetobia. Mg. pallipes. Mg. 58. A s in du l u m. Ltr. Platyurce. Ztt., MIg. Macrorrhyncha. Wtz. ol. femorale. $\mathrm{Mg}$. flavum. IVtz. rostratum. Ztt. nigrum. Ltr. fasciatum. Mg. 89. Platyura. Mg. Sciara. F. - Ceroplatus. F. - Asindulum. Ltr. marginata. $\mathbf{M g}$. atrata. F. flava. Mcq. 
modesta. Wtz. nigriceps. Wlk. humeralis. Wtz. intincta. Mg. fugax. Wtz. ochracea. Mg. fasciata. Ltr. infuscata. Wtz. discoloria. Mg. unicolor. Staeg. pallida. Staeg. aestivalis. Wtz. similis. Wtz. basalis. Wtz. discoidea. IVtz. selecta. Wtz. taeniata. Wtz. bicolor. Meq. semirufa. Mg. cincta. WVtz. succincta. Mg. nemoralis. Mg. nana. Meq. nigricolnis. F. Baumhaueri. Mg. fulvipes. $\mathrm{Mg}$. flavipes. Mg: erythrogaster. $\mathbf{M g}$. signata. Mlg. ruficollis. Mg: rufipes. Mg. vitripemis. Mg. pallipes. Meq. brummipennis. Staeg. dorsalis. Staeg. ruficornis. Ztt. nigriventris. Ztt. lugubris. Ztt. zonata. Ztt. servula. WVlk. mycetophiloides. Wlk. vitripennis. Wlk. antica. Wlk. micolor. TWlk.

concisa. WVlk.

60. Ceroplatus. Bosc. - Rhagio u. Sciara.

F. - Musca. Gm. Platyura. Mg.

lineatus. F.

striatus. Gm.

laticornis. $\mathrm{Mg}$. tipuloides. Bose. sesioides. Thhlbg: testaceus. Dalm. dispar. L. Duf. Reaumuri. L. Duf. humeralis. Ztt. atricornis. Ztt.

III. S i m u l i d a e.

61. Simulia. Ltr. Culex. L. - Tipula. Deg. - Scatopse. F. Fll. - Rhagio. F. Atractocera. Mg. ol. nigra. $\mathrm{Mg}$. varia, Mg. ornata. Mg.

regelationis. $\mathrm{Mg}$. ol. sericata. $\mathrm{Mg}$. reptans. L.

ervithrocophala. Deg. columhirschensis. F. sericea. I. cleyans. $\mathbf{M g}$. maculata. F. variegata. Mg. cincta. $\mathrm{Mg}$. posticata. $\mathrm{Mg}$. argyropeza. Mg. argyreata. Mg. nana. Ztt. latipes. Mg. auricoma. $\mathrm{Mg}$. aurea. Fries. maculata. Mg. pungens. $\mathrm{Pz}$. marginata. Mg. fuscipes. Fries. puliventris. Ztt. lineata. Mg. mymated. Ztt. columbatczensis. Schub. hirtipes. Fries. rufipes. Mg. rufia. $\mathrm{Mg}$. pusilla. Fries. pallipes. Fries. caua. $\mathrm{Mg}$. incana. Lw. subfasciata. Mg. picta. Mg. fasciata. Mg. verna. Meq. tibialis. Meq. crassitarsis. Meq. cinerea. Mcq. vittata. Ztt. annulitarsis. Ztt. minutissima. Ztt. humeralis. Ztt. ferruginea. Whlbg: lorealis. Ztt. fasciata. Curtis.

\section{Bibionidae.}

Scatopsinae.

62. Anarete. Hal. -

Lestremia. Mg. -

Sciara. Ztt. canditata. Hal.

al'sipennis. Mg. albipennis. Lw. coracina. Ztt. 63. Sc a tops e. Geoffr. Tipula. L. Deg. H. Ceria. Scop. - Aspistes. Ruthe. 
inermis. Ruthe. soluta. Lw.

halterata. $\mathrm{Mg}$. albipennis. v. Ros. minuta. Ztt. ol.

flavicollis. $\mathrm{Mg}$. ochracea. $\mathrm{Mg}$. claripes. Lw. scutellata. Lw. breticomis. Mg. pulicaria. Lw. notata. L.

albipennis. $F$. latrinarum. Deg. punctata. $\mathrm{Mg}$. nigra. Geoffr. leucopeza. Mg. nigra. $\mathrm{Mg}$. minuta. $\mathrm{Mg}$. vernalis. $\mathbf{M g}$. picea. $\mathrm{Mg}$. flavipes. Mg: fuscipes. Mg. glabra. Mg. femoralis. $\mathrm{Mg}$. recurva. Lw. transversalis. Lw. picipes. $\mathrm{Mg}$. nigripemis. Mg. major. Mcq. fulvitarsis. Meq. fuscinervis. Lw. infumata. Hal. albitarsis. Ztt. flavitarsis. Ztt. tristis. Ztt. geniculata. Ztt. bifilata. Wlk. consimilis. Wlk. integrata. Wlk. simplex. Wik. 6'. A spistes. Mg. berolinensis. $\mathrm{Mg}$. pullus. Wlk. borealis. Lw.

Haworthi. Curtis.

65. Corynoscelis. Boh. eximia. Boh.

\section{Bibioninuo.}

(66. Dilophus. Mg. Tipula. L. - Hirtea. F. - Bibio. Oliv. vulgaris. Mg. febrilis. L. forcipatus. Schrk. spinatus. Müll. termatus. Lw. femoratus. Mg. albipennis. Mg. tenuis. $\mathrm{Mg}$. humeralis. Ztt. marginatus. Mg. antipedalis. $\mathrm{Mg}$.

67. B i b i o. Geoflir. -

Tipula. L. - Hirtea.

F. Fll. Ztt. pomoliae. F. marci fulvipes. Deg. marci. L.

bievicornis. L. febrilis. Schrk. hortulanus. L. siculus. Lw. fulviventris. Mcq. venosus. Mg. nervosus. Mg. ol. reticulatus. Lw. nigriventris. Hal. hybridus. Hal. leucopterus. $\mathrm{Mg}$. Johamnis。 L. pyri. $\mathrm{E}$. praecox. F. pomonae. Schrk. hyalinus. Mg. ol. rufipes. $\mathrm{Mg}$. albipennis. Mg. lacteipennis. Ztt. clavipes. $\mathbf{M g}$. Johannis. F. doisalis. $\mathrm{Mg}$. flavicollis. Mg. ephippium. Ztt. clavipes. Hal. varipes. $\mathrm{Mg}$. rufitarsis. Mg. ferruginatus. L. flavicaudis. Deg. laniger. $\mathrm{Mg}$. vernalis. Mg. femoralis. $\mathrm{Mg}$. fulviventris. $\mathrm{Mg}$. villosus. Mg: nigripes. Mg. globuliceps. Lw. picinitarsis. Brullé. fulvipes. Ztt. umbellatarum. Ztt. festinans. Ztt. fulricollis. Gimmerth. 68. Penthetria. Mg. holosericea. Mg. funebris. $\mathrm{Mg}$.

Pachyneurinae.

69. Pachylleura. Ztt fasciata. Ztt.

70. Spodius. Lw. imbecillus. Lw.

\section{B. Polyneura.}

\section{Chir o 0 mill a e.}

71. Coryuoneura. Wtz. minuta. Wtz. minutissima. IVIk. 
scutellata. Wtz. celeripes. IVtz. atra. Wtz.

72. H y d robaenus. Fries. - Chivonomus. Mg. - Psilocenus. Ruthe.

lugubris. Fries. occultans. Mg.

73. Chironom us. Mg. - Tipule. L.

Thalassomyia. Schin. pt.

Frauenfeldi. Schin. punctipes. W.

dimidiatus. $\mathrm{Mg}$. abdominalis. Staeg. laetipes. Ztt. flavipes. Mg. gmundensis. Egg. vernus. $\mathrm{Mg}$. pusio. Mg. latus. Staeg. aftinis. W. viridulus. Mg. ol. flavellus. Ztt. hilarellus. Ztt. tenuis. Mg. niveipennis, $F$. pilicomis. $\mathrm{Mg}$. rudis. Fries. ojecurus. Ztt. ol. nubeculosus. Mg. flexilis. L. gibbus. F. fascipennis. Ztt. rutipes. $\mathrm{L}$.

bifasciatus. Mg. pulcier. Ztt. ol. quadrimaculatus. Mg. scalaenus. Schrk. histrio. F. sticticus. F. pictulus. $\mathrm{Mg}$. albofasciatus. Staeg: maculipennis. Mg. lugubris. Ztt. plumosus. L.

annularis. Ltr. grandis. Mg. intermedius. Staeg. prasinus. $\mathrm{Mg}$. pallens. $M g$. barbipes, Staeg. anthracinus. Ztt. annularius. Deg. Mg. annularis. Deg. aprilinus. $\mathbf{M g}$. ferrugineovittatus. Ztt. flaveolus. Mg.

flavicollis. $\mathrm{Mg}$. riparius. Mg. zonulus. 'Ztt. tentans. F. vernalis. Mg. ol. abdominalis. $\mathrm{Mg}$. venustus. Fries.

Waldheimii. Gmrth. Dahlbomi. Ztt. psittacinus. Mg. dispar. Mg.

lucidus. Ztt. productus. Ztt. albimanus. Mg. annularis. Mg. ol. pullus. Ztt. chloris. $\mathrm{Mg}$. niveipes. Ztt. dorsalis. Mg. tendens. F. viridis. $\mathrm{Mg}$. virens. L. vulneratus. Ztt. brevitibialis. Ztt. virescens. $\mathrm{Mg}$. virgineus. Schrk. viridulus, L.

littoralis. $\mathrm{Mg}$. of. viridulus. Mcc. pusillus. L. pedellus. Deg: fuscus. L. pt. cantans. F. litoralis. Schrk. pedestris. Mg. hyperboraeus. Staeg. annularis. Ztt. ol. Rosenschöldi. Ztt. cingulatus. Mg. nemoralis. Ztt. straminipes. Ztt. sociellus. Ztt. tenellus. Ztt. brumnipes. Ztt. litorellus. $\mathrm{Mg}$. plebejus. Mg: dubius. Ztt. altipes. Ztt. picipes. $\mathbf{M g}$. signatus, v. d. WIp. marmoratus. v. d. Wlp. blandus. v. d. Wip. unicolor. v. d. Wip. viridicollis. v. d. WIp. elegans. Mg. crassipes. Pz. aestivus. Curtis. hirtipes. Mcq. incomptus. Ztt. fuscipes. $\mathbf{M g}$. pallidicollis. Staeg. hirticollis. Staeg. immundus. Ztt. fuscus. $\mathrm{Mg}$. atratulus. Ztt. capucinus. Ztt. coracinus. Ztt. albipennis. Mg. melancholicus. Ztt. 
leucópogon. $\mathrm{Mg}$. gracillimus. Ztt. sordidellus. Ztt. minutus. Ztt. atomarius. Ztt. albolineatus. Mg. glabricollis $\mathrm{Mg}$. halteratus. Ztt. nigroviridis. Mcq. venosus. Mg. notatus. Mg. nigrimanus. Staeg. nerrosus. Staeg. prasinatus. Staeg. ictericus. Mg. longipes. Staeg. ruforittatus. Staeg. testaceus. Mcq. fraterculus. Ztt. confinis. Staeg. femoratus. Staeg. armillatus. Staeg. biannulatus. Staeg. incisuratus. Ztt. intersectus. Staeg. tibialis. Mg. ephippium. Ztt. obscurimanus. Ztt. pilitarsis. Ztt. pietipes. Ztt. assimilis. Ztt. bicinctus. $\mathrm{Mg}$. dizonias. $\mathrm{Mg}$. tricinctus. $\mathrm{Mg}$. trifasciatus. Pz. tremulus. L. motitator. L. Mg. motitatrix. L. annulipes. $\mathbf{M g}$. iylvestris. F. vibratorius. $\mathrm{Mg}$. triannulatus. Mifq. marginatus. Meq. ornatus. Mlg. barbicornis. F. L. obscurus. Mg. stercorarius. Deg. chiopterus. Mg. ol. byssinus. Schrk. aterrimus. Mg. minimus. Mg. flaronervosus. Staeg. lucens. Ztt.

lucidus. Staeg. brevitarsis. Ztt.

brevicornis. Ztt. modestus. Mg. flabellatus. Mg. alpicola. Ztt. pubitarsis. Ztt. frigidus. Ztt. variabilis. Staeg. perniger. $Z$ tt. bicolor. Ztt. von $\mathrm{Mg}$. bipunctellus. Ztt. varians. Staeg. nigriventris. v. d. Wlp. dilatatus. v. d. Wlp. sylvaticus. v. d. Wlp. ochraceus. v. d. Wlp.

geniculatus. F. pilicornis. F. pilipes. Mg. glaucus. Mg. olivaceus. $\mathrm{Mg}$. thoracicus. Mg* vitripennis. $\mathrm{Ng}$. scutellatus. Mg. terminalis. $\mathbf{M g}$. fuscipennis. Mg. rubicundus. Mg. pygmaeus. Mg. nitidus. $\mathrm{Mg}$. laetus. Mg. melaleucus. $\mathbf{M g}$. carbonarius. $\mathrm{Mg}$.

fasciatus. $\mathbf{M g}$. o scillator. $\mathrm{Mg}$. tristis. Mg. praecox. $\mathrm{Mg}$. junci. $\mathbf{M g}$. nanus. Mg. albidus. Mg. obscuripes. Mg. sulphuricollis. $\mathrm{Mg}$. flavimanus. $\mathbf{~} \mathrm{g}$. lividus. $\mathrm{Mg}$. lepidus. Mg. supplicans. Mg. opacus. $\mathrm{Mg}$. perennis. Mg. leucopus. Mg. rufiventris. $\mathrm{Mg}$. proximus. Mg. fuscimanus. Mg. amasia. Mg. pulchellus. $\mathrm{Mg}$. festivus. Mg. gibbosus. Mg. confinis. Mg. nubilus. $\mathrm{Mg}$. sellatus. Mg. pallidulus. Mg. debilis. $\mathrm{Mg}$. longimanus. Mg. albipes. Mg. rusticus. Mg. nubilipennis. Mg. albicornis. Mg. scriptus. $\mathrm{Mg}$. bicolor. $\mathrm{Mg}$. paganus. $\mathrm{Mg}$. intersectus. $\mathrm{Mg}$. minutissimus. Mq. amoenus. $\mathrm{Mg}$. rivularis. $\mathrm{Mg}$. denigrator. ragans. $\mathrm{Mg}$. 
indulgens. $\mathrm{Mg}$. gracilis. Meq. viridipes. Meq. temuis. Mcq. non Mg. unifasciatus. MLcq. ruficollis. Meq. pallidus. Meq. notabilis. Meq. nigrinus. Meq. maculosus. Meq. humeralis. Mcq. ammulatus. Meq. viridanus. Ruthe. albocinctus. Gimmerth. virens. Gimmertll. nigripes. Gimmerth. livonensis. Gimmerth. basalis. Staeg. adjunctus. Wik. affinis. Wlk. alligatus. Wlk. appositus. Wrik. cingulatus. Wlk. coaequatus. Wlk. compertus. WVlk. contingens. Wlk. convectus. Wlk. convictus. Wlk. conversus. Wlk. convestitus. WVlk. denotatus. WVlk. deproperans. Wlk. disclusus. Wlk. discrepans. Wlk. dispessus. Wlk. dissidens. Wlk. divisus. Wlk. dolens. WVlk. effusus. Wlk. ejuncidus. WVlk. eminulus. Wlk. ennotatus. Wlk. expalpans. Wilk. expatiens. Wlk. fertus. Wlk. futilis. Wlk. habilis. WIk. hebescens. Wlk. imbecilis. Wlk. impar. Wlk. impensus. Wlk. incallidus. Wlk. incertus. Wlk. incoactus. Wlk. incomptus. Wik. inopertus. WVlk. inscendens. Wlk. inserpens. Wik. interseptus. Wlk. intextus. Wlk. intrudens. IVlk, involitans. Wlk. irretitus. Wlk. irritus. WIk. lentulus. Wlk. malacus. Wlk. maneus. Wlk. marcidus. Wlk. minusculus. Wlk. modicellus. Wlk. moerens. WVIk. morulus. WV]k. moturus. Wlk. nactus. Wlk. nexilis. Wlk. nigratus. Wlk. nitidicollis. WVIk. notescens. Wlk. novatus. Wlk. nugax. Wlk. obditus. Wlk. objectans. Wlk. oblidens. Wlk. obnixus. Wlk. obreptans. Wlk. obsepiens. Wlk. obsistens. THIs. obtexens. WVlk. obvertens. Wlk. obrius. Wlk. occipiens. Wlk. offectus. Wlk. opplens. Wlk. oppertus. Wlk. oppetitus. Wlk. paganicus. Wlk. pallidicornis. Wlk. parilis. Wlk. patens. Wlk. patibilis. Wlk. perexilis. Wlk. pergens. WVlk. perlevis. Wlk. permacer. Wlk. perpessus. Wlk. pereptans. Wlk. persidens. Wlk. pertenuis. Wlk. pertractus. Wlk. pervulsus. Wlk. pulsus. Wlk. tarsalis. Wlk. trichopus. Wlk. tristus. Wlk.

rufovittatus. Wlk. ol. zonarius. WIk.

74. Tanypus. Mg. Tipula. L. - Chironomes. F. nervosus. Mg. nigrimanus. Mg. fasciculatus. F. punctipennis. Mg. cinctus. $\mathrm{Pz}$. culiciformis. L. choreus. Mg. fasciatus. Mg. ol. sylvaticus. Mg. ol. plumipes. Fries. 
forcipatus. Egg.

nudipes. Ztt.

nebulosus. $\mathrm{Mg}$.

litoralis. Mg.

varius. $\mathrm{F}$.

punctatus. $\mathbf{M g}$. ol.

punctatus. F.

nebulosus. Mg. ol.

ornatus. Mg.

carneus. $\mathrm{E}$.

albipes. Fries.

monilis. L.

maculatus. Deg.

phatta. Egg.

signatus. Ztt.

binotatus. W.

melanops. Mg.

bicolor. Fries.

arundineti. L.

nigropunctatus. Staeg.

zonatus. F.

flabellicornis. F.

notatus. $\mathrm{Mg}$.

praecox. $\mathrm{Mg}$.

pusillus. Mg.

dubius. Mg.

ferruginicollis. $\mathrm{Mg}$.

tanypodipennis. Ztt. pallidulus. Mg.

rufus. $\mathrm{Mg}$.

incarnatus. Mg.

nubilus. Mg.

laetus. Mg.

festivus. Mg.

melanurus. Mg.

gratus. $\mathrm{Mg}$.

tenuis. $\mathrm{Mg}$.

ciliatus. Mg.

ater. $\mathrm{Mg}$.

bilineatus. Mg.

fasciatus. Mcq.

unimaculatus. Micq.

hirsutus. Meq. obscurus. Meq.

lentiginosus. Fries.

anulatus. Staeg.

flabellicornis. Mg.

dubius. Staeg.

longimanus. Staeg:

tibialis. Staeg.

turpis, Ztt.

consobrinus. Ztt.

crassinervis. Ztt.

lacteipennis. $Z$ tt.

morio. Ztt.

pubitarsis. Ztt.

lugubris. Ztt.

barbitarsis. Ztt.

pallicornis. Ztt

choreus. Fries.

sordidus. Ztt.

maculipennis. Ztt.

unifascipennis. Ztt.

trifascipennis. Ztt.

luteus. Gimmerth.

griseipennis. v. d. Wlp.

guttipennis. v. d. Wlp.

73. D iamesa. Mg. -

Chironomus. Wlk.

Gaedii. Mig:

notata. Staeg.

Waltlii. $\mathbf{M g}$.

Ammon. Wlk.

Chiron. IVlk.

tonsus. Wlk.

Typhon, Wlk.

76. Clunio. Hal.

marinus. Hal.

adriaticus. Schin.

77. Ceratopogon. Mg. - Tipula. L. - Culex. L. - Chironomus. F. - Culicoides. Ltr. - Sphaeromyas. Curt.

pallidus. Wtz. regulus. Wtz.

niger. Wtz.

myrmecophilus. Egg.

trichopterus. Mg.

bipunctatus. $\mathrm{L}$.

trichopterus. Mg. ol.

hirsutulus. Ztt.

costatus. Ztt.

trichopterus. Wtz.

Kaltenbachii. Wtz.

piceus. Wtz.

ciliatus. Wtz.

crassipes. Wtz.

lepidus. Wtz.

brevipennis. Mcq.

tenuis. Wtz.

divaricatus. Wtz.

alacer. Wt\%.

murinus. Wtz.

titillans. Wtz.

saltans. Wtz.

halteratus. IVtz.

velox. Wtz.

frutetorum. Wtz.

sylvaticus. Wtz.

forcipatus. Wtz.

lucol um. Mrg.

rostratus. Wtz.

brunnipes. Mg.

pavidus. Wtz.

fuscus. Mg.

minutus. $\mathrm{Mg}$.

sericatus. Wtz.

amoenus. Wtz.

varius. Wtz.

fascipennis. Staeg.

pictipennis. Staeg.

arcuatus. Wtz.

obsoletus. Mg.

nubeculosus. $\mathrm{Mg}$.

pulicaris. L.

punctatus. Mg.

albicans. Wtz. 
aestivus. Wtz. cunctans. Wtz. modestus. Wtz. egens. Wtz. scutellatus. Mg. versicolor. Wtz. obscurus. Wtz. pumilus. Wtz. neglectus, Wtz. splendidus. Wtz. ochraceus. Wtz. vitiosus. Wta. lacteipennis. Wtz. nitidus. Wtz. flavirostris. Wtz. illustris. Wtz. niveipennis. Mg. copiosus. Wtz. canditatus. Wtz. flavipes. Mg.

hortulanus. Mg. spinipes. $\mathrm{Mg}$ elegans. Wtz. variegatus. Wtz.

? unimaculatus. Mcq. ferrugineus. Mg. serripes. Mg. rufipectus. Wtz. lineatus, Mg*. tibialis. Mg. fasciatus. Mg. cinyulatus. Mg. marginatus. Curtis. canditatus. Lw. procerus. Ztt. leucogaster. Ztt. posticatus. Ztt. femoratus. $\mathbf{M g}$. F. morio. E. palustris. $\mathrm{Mg}$. inflatus. $\mathrm{MIg}$. valyatus. ITtz. gracilipes. Wtz. rubiginosus. Wtz. bicolor. $\mathrm{Pz}$.

venustus. $\mathbf{M g}$. concinnus. $\mathbf{M g}$. siguatus. Mg. gracilis. IVtz. ornatus. Mg. albipes. Wtz. solstitialis. Wtz. nobilis. Wtz. flavipalpis. Wtz. subsultans. Schrk. vittatus. IV. holosericeus. Mg: Horalis. Mg. leucopeza. Mg. albitarsis. W. communis. F. lencopterus. $\mathrm{Pz}$. albipennis. $\mathbf{M g}$. ambiguus. $\mathrm{Mg}$. stigma. Mg. albicornis. Mg. pallipes. $\mathbf{M g}$. nemorosus. Mg. pictus. $M g$. rufipes. Mg. armatus. Mg. rufitarsis. Mg. succinctus. Mg. terreus, Mg. fuliginosus. Mg. funebris. Mg. chiopterus. Mg. tristis. Mg: punctatus. Mg. pratensis. $\mathbf{M g}$. anmulipes. $\mathrm{Mg}$. maculipes. Mg. nigripes. $\mathbf{M g}$. annulatus. $\mathbf{M g}$. glabricollis. $\mathbf{M g}$. lateralis. Mg: varipes. $\mathbf{M g}$. melancholicus. Mg. luteitarsis. Mg. armipes. $\mathbf{M g}$. hortulanus. Ztt. flavitarsis. $\mathbf{M g}$. Foersteri. $\mathrm{Mg}$. ruficornis. Mcq. univittatus. Ztt. nigritulus. Ztt. humeralis. Ztt. sordidellus, Ztt. tessellatus. Ztt. vittiger. $Z$ tt. fuscipes. Ztt. villosus. $Z t t$. ralidinervis. Ztt. coracinus. Ztt. pusio, Ztt. pennicornis. Z $/$ tt. minutissimus. Ztt. lugubris. Ztt.

bipunctatus, Ztt. ol. flavoscutellatus, Ztt. griseolus. Ztt. ephippium. Ztt. tarsatus. Ztt. vexans. Staeg. aftinis Staeg. binotatus. Staeg: cinereus. Mcq. circumdatus. Staeg. erythrocephalus. Staeg. transfuga. Staeg: flavicornis. Staeg. bracbialis. Hal. distinctus. Hal. gracilis. Hal. dorsalis. Ztt. flavifrons. Guér. geniculatus, Guér. brumnipes. Perris. flaripes. Gimmerth. 
barbipes. Gimmerth. aristolochiae. Rond. taeniatus. Wlk. calceatus. WVIk.

VI. Psychodidae.

78. Sycorax. Curtis.

Posthon. Lw.

silacea. Curtis.

79. Trichomyia. Curtis. - Psychoder. Ztt. urbica. Curtis.

aurea. Ztt.

80. Ulomy ia. Wik. -

- Saccopteryx. Hal.

Tipula. L. - Tricho-

ptera. Mg. - Psy-

clioda. Mg:

fuliginosa. Mg.

lirta. L.

81. Psychoda. Ltr. -

Tipula. L. - Bibio.

Gffr. - Trichoptera.

Mg. ol.

phalaenoides. L.

muraria. Ltr.

nervosa. Mg.

sexpunctata. Curtis.

phalacnoides. Mg.

albipennis, Ztt.

humeralis. Mg.

82. Pericoma. Curtis.

- Trichoptera. Mg.

ol. - l'sychoda. Auct.

palustris. Mg.

auriculata. Curtis.

ocellaris. $\mathbf{M g}$.

canescens, Mg.

nubila. Mg.

phalaenoides. Scop.

bomlyciformis. Schrk.

tristis. MIg.

calceata. Mg.

trifasciata $\mathrm{Mg}$. tusca. Meq.

phalaenoides. Schrk.

lucifuga. Wlk.

soleata. Wlk.

bullata. WIk.

ustulata. iTlk.

83. Phlebotom us. Rond.

- Bibio. Scop. -

Haemasson. Lw. -

Cyniphes. Costa.

papatasii. Scop.

minutus. Lw.

molestus. Costa.

minutus. Rond.

VII. Culicidae.

84. Corethra. MIg. -

Tipula. Deg. F. -

Chironomus. F. -

Erioptera. Ztt. pt. culiciformis. Deg.

pallida. F.

plumicornis. $\mathrm{F}$.

cristallina. Deg.

pilicornis. $\mathbf{F}$.

lateralis. Ltr. $\mathrm{Pz}$.

hafniensis. Gm.

flavicans. Mg.

fusca. Staeg.

culiciformis. $\mathbf{M g}$.

rufa. Ztt.

Nyblaei. Ztt.

obscuripes. $\mathrm{r}$. d. $111 \mathrm{p}$.

pilipes. Gimmerth.

85. Mochlonyx. Lw. -

Corethra. Ruthe.

velutina. Ruthe.

effoetus. Wik.

86. A ëdes. Mg.

cinereus. Mg.

rufus. Gimmerth.

ก. Anopheles. Mg: -

Culex. I. F. bifurcatus: L.

trifurcatus. F.

claviger. Mg. ol.

plumbeus. Hal.

maculipennis. $\mathrm{Mg}$.

bifurcatus, $\mathrm{Mg}$. ol.

nigripes. Staeg.

pictus. Lw.

villosus. R. Desv.

88. Culex. L.

annulatus. Schrk. F. variegatus. Schrk.

dorsalis. Mg.

vexans. Mg.

cantans. $M g$.

maculatus. Mg.

annulipes. MIg.

bicolor. $\mathrm{Mg}$.

nemorosus. Mg.

reptuns. $\mathrm{Mg}$. ol.

fasciatus. $\mathbf{M g}$. ol.

sylvaticus. Mig.

guttatus. Curtis.

pipiens. L.

vulyaris. L.

ciliaris, L.

rufus. Mg.

glaphyropterus. Schin.

ornatus. Mg.

equinus. MIg. ol.

lateralis. Mig.

domesticus. Grm.

punctatus, Mg.

flarirostris. Mg.

sticticus. Mg.

calopus, $\mathrm{Mg}$.

pallipes. $\mathbf{M g}$.

quadrimaculatus. Micq.

bipunctatus. Mcq.

parvus Micq.

Kounopi. Brullé.

detritus. Hal.

fumipennis. Steph. 
nigripes. Staeg.

nigritulus. Ztt.

fusculus. Ztt.

vittatus. Bigot.

lutescens. $\mathrm{F}$.

rusticus. Rssi.

stercoreus. L. Vill.

VIII. Tipulidae.

Anomalae.

89. Chionea. Dalm. araneoides. Dalm. crassipes. Bohem.

Limnobinae.

90. Erioptera. Mg. - flavipennis. Staeg.

Tipula. F. - Li-92. Dasyptera. Schin.

monia. Ltr. - Molophilus. Curtis. pt. atra. $\mathrm{Mg}$.

Tip. erioptera. F.

brevipennis. Curt.

murina. Mg.

pygmaea. Mcq.

obscura. Mg.

grisea. Mg.

ochracea. Mg.

crassipes. Curtis.

appendiculata. Staeg. propinqua. Egg.

pallipennis. Mcq.

icterica. Egg.

imbuta. Mg.

flavescens. L.

lutea. Gmel.

lutea. Mg:

taenionota. Mg.

analis. Ztt. ol.

montana. Mg.

fuscipennis. Mg.

griseipennis. Mg.

lateralis. Mcq.

nigra. Mcq.

opaea. Mg.

denudata. Ztt.

sordida. Ztt.

obscuripes. Ztt.

- Erioptera. Auct.

- Ormosia u. Spy-

lopter $\alpha$. Rond.

varia. $\mathrm{Mg}$.

haemorrhoidalis. Ztt.

sordida. Ztt. ol.

diuturna. W'lk.

distincta. Egg.

lineata. Mg.

nodulosa. Meq.

hederae. Curtis.

ruficauda. Ztt.

fascipennis. Ztt.

similis. Staeg.

91. Trichosticha. Schin 93. Rhypholophus.

- Tipula. L. -

Erioptera. Auct. -

Limnobia. Auct. pt.

Ilisia, Ilisophila u.94. Gonomyia. O-Sak.

Chemalida. Rond.

maculata. Mg.

trivialis. Mg.

cinerascens. $\mathrm{Mg}$.

ciliaris. Schum.

sericea. Sicq.
Kolenati.

phryganopterus. Kolenati.

- Limnobia. Auct. pulchripennis. Lw.

? semyuttata. Hal. tenella. Mg. scutellata. Egg. cincta. Egg.

schistacea. Schum.

diluta. Ztt.

flava. Schum.

nubila. Schum.

cothurnata. MIcq.

gracilis. Ztt.

inconclusa. Wlk.

95. Symplecta. Mg. -

Limnobia. Auct. -

Helobia. St. Farg.

stictica. Mg.

punctipennis. $\mathrm{Mg}$.

hybrida. Mg. ol.

similis. Schum.

stictica. Ztt. ol.

96. Gnophomyia. O.Sak.

pilipes. $\mathrm{E}^{\prime}$.

fimbriata. Mg.

sylratica. Mg.

umbripennis. Schum.

vagans. Wlk.

errans. Wilk.

97. Psil ocollopa. Ztt.

Meigenii. Ztt.

98. A nis omera. Mg. -

Nemalocera. Mg. ol.

- Tipula. F. - He-

watoma. Ltr. - Pe-

renocera. Curtis.

striata. F.

bicolor. $\mathrm{Mg}$.

Gaedii, Mg.

obscura. Mg.

nigra. Ltr.

vittata. $\mathrm{Mg}$.

fuscipennis. Curtis.

99. Penthoptera. Schin.

- Tipula. Scop. chirothecata. Scop. cimicoides. Scop. 
100. Tricyphona. Ztt. - Limnobia. Auct. immaculata. $\mathrm{Mg}$.

101. Dicranota. Ztt. - Limnobia. Schum. bimaculata. Schum. demissa. Hal.

Guerini. Ztt. parida. Hal. ruficornis. Schum.

102. Ul a. Hal. - Limnobia. Auct. pilicornis. Ztt. sororcula. Ztt. pilosa. Schum. macroptera. Mcq. molissima. Hal.

103. Ama lopis. Hal. Limnobia. Auct. Crunobia. Kolenati. tipulina. Egg. littoralis. Mg. Schineri, Kolenati. gmundensis. Egg. opaca. Egg. occulta. NIg. geniculata. $\mathrm{Mg}$. unicolor. Schum. straminea. Mg.

104. Pedicia. Ltr. Tipula. L. - Limnobia. Mg. Schum. rivosa. L.

triangularis. $\mathrm{F}$. venosa. Wlk.

103. Trichocera. $\mathbf{M g}$. Tipula. L. - Limomia. Ltr.

maculipennis. Mg. cinerea. $\mathrm{F}$. regelationis. $\mathbf{L}$. annulata. $\mathrm{Mg}$.

hiemalis. Deg. parva. Mg. fuscata. MIg.

106. Ephelia. Schin. Limnobia. Auct. Limnophila. Mcq. marmorata. Mg. maculata. Mg. ol. decora. Hal. guttata. Mcq. miliaria. Egg. trimaculata. Ztt.

107. Idioptera. Meq. Tipula.F.L. - Limnobia. Auct. fasciata. L. pulchella. Mg. fasciata. Schum. maculata. Meq.

108. Limnophila. Mcq. - Limnobia. Auct. hospes. Egg. nemoralis. If leucophaea Ztt. ol. inclusa. Wik. obsoleta. Wlk. adjuncta. Wik. plebeja. Mg. leucophaea. Mg. bicolor. $\mathrm{Mg}$. phaeostigma. Schum. fuscipennis. Mg. lucorum. Mg. aberrans. Wlk. nitidicollis. Mg. discicollis. Mg. placida. Wlk. ferruginea. Mg. flavescens. Litr. praeusta. Schum. glabricula. Mg. binotata. Ztt. ol. punctum. Schum. longicornis. Schum. punctum. Mg. ochracea. Mg. lineola. Mg. fulvonerrosa. Schum. dispar. Mg. nigricollis. Mg. obsoleta. Mg. fulvescens. Mg. pratorum. Mg. placida. Mg. nigrina. $\mathrm{Mg}$. ruralis. $\mathrm{Mg}$. sessilis. Mcq. humilis. Mg. stigmosa. Mcq. pruinosa. Mcq. nigricans. Mcq. abdominalis. Staeg. scutellata. Staeg. arctica. Ztt. hyalipennis. Ztt. subcincta. Ztt. decolor. Ztt.

unicolor. Ztt. ol. squalens. Ztt. 109. Poecilostola. Schin. - Tipula. L. - Limnolia u. Limnophila. Auct. angustipennis. Mg. pictipennis. $\mathrm{Mg}$. punctata. Mg. ocellaris. L. barbipes. Nig. 110. Epiphragma. 0.Sak. - Tipula. F. Limnobia.Mg.Schum. - Limonia. Ltr. Limnophila. Mcq. Stg. 
picta. F.

ocellaris. Curtis.

111. Dactylolabis. O.Sak.-Limnobia. Mg.

- Limnophila. Meq.

dilatata. Lw.

Frauenfeldi. Egg.

terg*estina. Eg'g*

symplectoidea. Egg.

transversa. Mg.

sexmaculata. Mcq.

112. Elliptera. Schin.

omissa. Egg:

113. Antocha. O.-Sack. opalizans. O.-Sack.

114. Dicranoptycha.

o.-Sack. - Limno-

bia. Mg. Schum.

cinerascens. $\mathrm{Mg}$.

fuscescens. Schum.

115. Rhamphidia. Mg.

- Limnobia. Schum.

- Leptorlina. Steph.

- Megarhina und

Helius. St. Farg.

longirostris. $\mathrm{Mg}$.

bicolor. Steph.

inornata. Mg.

flava. Wlk.

116. Rhiphidia. Mg. -

Tipula. Schrk.

maculata. Mg.

punctata. Schrk.

uniseriata. Schum.

117. Geran o m y ia. Hal. - Aporosa. Meq.

unicolor. Hal.

118. Limnobia. Mg. -

Tipula, L. - Limonia. Ltr. - Glochina.Mg.pt. - Limnobia u. Dicranomyia. 0.-Sack. quadrimaculata. L.

calmariensis. L.

quadrinotata. Mg.

annulus. Mg.

quadrimaculata. Mg. ol.

nubeculosa. $\mathbf{M g}$.

flavipes. F.

analis. $\mathrm{Mg}$.

nebulosa. Ztt. ol.

sylvicola. Schum.

tripunctata. Ztt. ol.

affinis. Ztt. ol.

nigropunctata. Schum.

sexpunctata. F.

xanthoptera. Mg.

bifasciata. Schrk.

aurea. Miül.

tripunctata. $\mathrm{Mg}$.

phragmitidis. Schrk.

stigma. Mg*.

sexnotata. Schum.

terestris. L.

trivittata. Schum.

albifrons. MIg:

globata. W1k.

sera. Wilk.

ornata. Mg.

chorea. Mg.

dumetorum. Mg.

didyma. Mg.

trinotata. Mg:

modesta. Mg.

autumnalis. Staeg.

lutea. $\mathrm{Mg}$.

inusta. Mg.

macrostigma. Schum.

marginata. Meq.

stigmatica. Mg.

affunis. Schum.

croatica. Egg. morio. $\mathrm{F}$.

leucocephala. Schum.

angustipennis. Ztt.

pilipennis. $\mathbf{E g}$.

collaris. Mg.

tristis. Schum.

grisea. Meq.

ventralis. Schum.

atra. Meq.

pulla. Mg.

fiavescens. Mcq.

variegata. Mcq.

unimaculata. Mcq.

elegans. Ztt.

consimilis. Ztt.

decora. Ztt.

maculipennis. Mg.

pabulina. Mg.

simplex. Mg.

ritripennis. Mg.

fusca. Mlg.

sericata. Mg.

mitis. Mg:

nova. Mg.

quadra. Mg.

lateralis. Mcq.

favolimbata. Dale.

manifesta. IVlk.

laevigata. Mcq.

argentea. Mcq.

brunnipennis. Mcq.

binotata. Mg.

frontalis. Staeg.

meridiana. Staeg.

tenuipes. Ztt.

murina. Ztt.

hyalinata. Ztt.

oscillans. Hal.

kransversalis. Wik.

punctigera. Wlk.

glabrata. Wlk. 
tarda. Wlk.

turpis. Wlk.

annulata. L.

imperialis. Lw. caesarea. O.-Sack.

alpina. Ztt. virgo. Ztt. juvenilis. Ztt.

platyptera. Meq. varinervis. Ztt. stigmatella. Ztt. lugubris. $\mathrm{Ztt}$. bifurcata. Ztt. unicolor. Ztt. caelebs. Ztt. zonata. Ztt. secreta. Wlk. finitima. Wlk. filata. Wlk. disjuncta. Wlk. nigrirostris. Gimmerth. viridipennis. Gimmerth. sexguttata. Dale. longipennis. Schum. senilis. Hal.

119. T' riog ma. Schin. -

Limnobia. Schum. trisulcata. Schum.

120. Phalacrocera. Schin. - Tipula. L. - Limnobia. Auct. nudicornis. Schum. replicata. $\mathrm{L}$.

brevirostris. Ztt.

121. Cylindrotoma. Mcq. Limnobia. Mg. distinctissima. $\mathbf{M g}$. brevicornis. Ztt. ol. glabrata. Mg. nigriventris. Lw. diversa. Wlk.

Ptychopterinae.

122. Ptychoptera. Mg. - Tipula. L. contaminata. L. fuscipes. Gmel. albimana. F. ocreata. Schrk, lacustris. Mg. paludosa. Mg. fasciata. Wlk. scutellaris. Mg. nigra. $\mathrm{F}$. pectinata. Mcq.

Tipulinae.

123. Dolich opeza.Curt. sylvicola. Curtis.

chirothecata. Wlk.

124. Pachyrhina. Meq. - Tipula. Auct.

pratensis. L. imperialis. $\mathrm{Mg}$. scalaris. $\mathrm{Mg}$. flavipalpis. $\mathrm{IIg}$. crocata. L.

fasciata. Deg. annulicornis, $\mathrm{Mg}$. varicornis. Schum. lunulicornis. Schum. quadrifaria. $\mathrm{Mg}$. fascipernis. Ztt. analis. Schum. iridicolor. Schum. sannio. Mg. cornicina. Ztt. ol. scurra. $\mathrm{Mg}$. maculosa. Mg. maculata. Mg. ol. histrio. $\mathrm{F}$.

lineata. Scop.

flavomaculata. Deg.

cornicina. $\mathrm{Mg}$. ol.

maculosa. Ztt. ol.

cornicina. L.

quadristriata. Schum.

dentata. Mg.

luteata. Mg.

picta. Mg.

pieticornis. Ztt.

125. Nephrotoma. Mg.

- Tipula. F.

dorsalis. F.

126. Tipula. L. - Pri-

onocera. Lw. pt. Pterelachisus. Rond. pt.

nigra. $L$. verticillata. $\mathrm{F}$.

lutescens. F. fulvipennis. Deg. gigantea. Schrk. hortorum. Hbst. maxima. Poda. rivosa. Scop. sinuata. F. repanda. Lw. triangulifera. Lw. vittata. $\mathrm{Mg}$. tenuicornis. Schum. truncorum. Mg. pabulina. Mg. stigmosa. Meq. rufipennis. Mg. Winnertzii. Egg. heros. Egg. nerrosa. Mg. hortensis. Mg. hortorum. Mg. ol. hortorum. L. hortulana. Mg. 
luridirostris. Schum. favirostris. Staeg. varipennis. Mg. simplicicornis. Ztt. nigricornis. Meq. submarmorata. Schum. guttipennis. Mg. pictipennis. Staeg: micans. Ztt.

marmorata. Mg. signata. Staeg.

irrorata. Neq. crassicornis. Ztt. nodulicornis. Ztt. octolineata. Ztt.

septemlineata. Meq. rufina. Mg.'

longicornis. Schum. Ceres. Ztt.

Meyer-Düri. Eg'g。 excisa. Schum. speculum. Ztt. montana. Curtis. seripta. Mg. nubeculosa. Mg: griseofusca. Deg. hortorum. E. guttulifera. Ztt. unea. IV. obsoleta. Mg. rubripes. Schum. subunilineata. Ztt. verualis, $\mathrm{Mg}$. macrocera. Ztt.

longicomis. Ztt. ol. caesia. Schum. plumbea. F. fenestrata. Schum. marginata. Mg. lateralis. $M g$. montium. Egg. paludosa. $\mathrm{Mg}$. oleracea. I., fusca. Staeg.

melanoceros. Schum.

lineata. Staeg.

cervina. MIg.

grisescens. Ztt.

luteipennis. $\mathbf{M g}$.

nodicornis. $\mathbf{M g}$. juncea. Mg. pagana. Mg. plicata. Mg. ol. luridiventris, Ztt. dispar. Hal. autumnalis. Lw. livida. $\nabla . d$. ITlp. recticornis. Schum. flavolineata. $M g$. antennata. Schum. latevittata. Schum. pruinosa. II. Siebkei. Ztt. stigmatella. Sclium. Diana. Mg:

turcica. F. pubescens. Lw. serricornis. Ztt. subserricornis. Ztt. subnodicornis. Ztt. lunata. I. ochracea. $\mathrm{Mg}^{\text {. }}$

lunata. F. peliostigma. Schum. caudatula. Lw. mellea. Schum. affinis. Schum. Selene. Mg. dilatata. Schum. fascipennis. $\mathrm{Mg}$. laetabilis. Ztt. limbata. Ztt. picticornis, Ztt. clandestina. $\mathrm{Mg}$. lineola. $\mathrm{Mg}$. selenitica. Mg. fimbriata. Mg. irrorata. Meq. breviterebrata. Mcq. pusilla. Mcq. nana. Meq. fuliginosa. Gimmerth. lactipenuis. Gimmerth. limitata. Schum. quadrivittata. Staeg. humilis. Staeg. raga. WIk. Bertei. Rond.

127. Ctenophora. Mg. - Tipula. L. Tanyptera. Leach. Ctenophora, Dictenidia und Xiphura. Brullé. - Ceroctena, Xiphrora und Ctenophora. Rond.

bimaculata. L. pectinata. Gmel. paludosa. E. ruficornis. Mg. atrata. L.

ichneumonea. Deg. ruficornis. Staeg.

Villaretianc. Brullé. nigricornis, Mg. atrata. Mg. ol. nigrofasciata. Mcq. pectinicornis. L. nigrocrocea. Deg. variegata. $\mathrm{F}$. flaveolata. F. crocata. Schrk. variegata. Poda. guttata. Mg*. ornata. $\mathrm{Mg}$. festiva. $\mathbf{M g}$. elegans. $\mathbf{M}$ g.

pectiniconis. $\mathrm{E}$. 
flaricornis. Mg.

vittata. Mg.

Ix. Rhyphidae.

128. Rhyphus. Ltr. Tipula. Scop. Ilusca. L. - Sciara und Rhayio F. Anisopus. Mg. ol.

fenestralis. Scop. succinctus. Gnel. cinctus. F. fenestralis. Curtis. ochraceus. Curtis.

fuscatus. F.

fuscus. Mg.

punctatus. F.

nebulosus. Mg. ol.

bilineatus. Gmel.

nigricuns. L.

minor. Ztt.

Generaincertaesedis.

129. Corynocera. Ztt. - Tanypus. Ztt. ol. ambigua. Ztt.

crassipes, Ztt. ol.

130.Blepharicera. Mcq.

- Asthenia. Westw.

- Liponeura. Lw.

- Apistomyia. Bigot. fasciata. Westw.

limbipennis. Mcq. cinerascens. L.W.

elegans. Bigot.

131. Macropeza. Mg. albitarsis. Mg.

132. Orphnephila. Hal.

- Chenesia. Meq. -

Thaumalea. Ruthe. testacea. Ruthe.

devia. Hal.

obscura. Ztt.

tarda. Lw.

133. Dixa. Mg.

aprilina. Mg.

cincta. Curtis.

fuliginosa. Wlk.

lineata. $\mathrm{Mg}$.

maculata. $\mathrm{Mg}$.

moesta. Curtis.

serotina. Mg.

nebulosa. Mg.

nubilipennis. Curtis. humilis. Ruthe.

nigra. Staeg.

obscura. Lw.

laeta. Lw.

puberula, Lw.

aestivalis. $\mathrm{Mg}$.

ritripennis. Mg.

autumnalis. $\mathrm{Mg}$.

\section{B. Brachycera.}

\section{a. Cyclocera.}

\section{Stratiomydae.}

Pachygastrinae.

134. Pachygaster. Mg.

- Nemotelus. Pz. -

Sargus. kll. - Vappo. F.

ater. $\mathrm{F}$.

Leachii. Curtis.

pallipennis. Meq. tarsalis. Ztt.

meromelas. L.-Duf.

minutissimus. Ztt.

orbitalis. Whlbg.
Stratiomynae.

135. Ne m otelus. Geoffr.

- Musca.L. - Stra-

tiomys. F.

pantherinus. L.

marginatus. F.

marginelless. Gmel.

Pz.

uliginosus. Ltr. $\mathrm{Pz}$.

muticus. Schrk.

fraternus. Lw.

nigritus. Mg.

brevirostris. Mg.

brachystomus. Lw.

globuliceps. Lw.

uliginosus. Lw, ol.

uliginosus. L.

muticus. $\mathrm{F}$.

bifasciatus. M.

pica. Lw.

plagiatus. Schin.

luteicornis. Egg.

notatus. Ztt.

nigrinus. Fll.

nigritus. $\mathrm{Pz}$.

signatus. Frivaldsk.

crenatus. Eg.g.

proboscideus, Lw.

punctatus. F.

longirostris. $\mathbb{H}$.

lasiops. Lw.

anchora. Lw.

lateralis. L.-Duf.

cingulatus. L.-Duf.

atriceps. Lw.

nigrifrons, Lw.

argentifer. Lw.

aerosus. Gimmerth.

ventralis. MIg.

paludosus. $\mathrm{Mg}$.

frontalis. Meq.

limbatus. Egg. 
136. Lasiopa. Brullé. - Nemotelus. F. -Clitellaria. $\mathrm{Mg}$. Cyclogaster. Meq. villosa. $\mathrm{F}$. calva. Mg. Peleteria. Brullé. tenuirostris. Lw.

137. Clitellaria. Mg. - Odontomyia. Trlk. - Ephippium. Mcq. Dahlii. Mg.

balius. Wlk. pacifica. Mg.

138. Ephippium. Ltr. - Musca. Schäfi. Clitellaria. Mg. Strationys. F. Pz.

thoracicum. Ltr. ephippium. F. inda. Don.

139. Oxycera. Mg. Musca. i. - Stratiomys. F. Pz.

trilineata. F. pantherina. L. pt. analis. $\mathrm{Mg}$. terminata. Mg. leonina. $\mathrm{Pz}$. pygmaea. Fll. muscaria. TVlk. affinis. Dall. formosa. IV. muscaria. Mg. Pz. nigricomis. En. $\mathrm{m}$. pardalina. $\mathrm{Mg}$. formosa. Meq. muscaria. F. flavipes. Lw. Ranzonii. Schin. Morrisii. Curtis. amoena. Lw. pardalina. Ztt. pulchella. Mg. hypoleon. $\mathrm{Mg}$. ol. $v^{*} v^{*} \alpha$. Wrlk.

Meigenii. Staeg. hypoleon. F.

Fallenii. Staeg. hypoleon. Fll. Ztt. locuples. Lw. dives, Lw. hypoleon. Schrk. hypoleon. L. tenuicornis. Mcq. longicornis. Dale. terminata. IVIk. nigra. Mcq. maculata. Ztt. marginata. Lw. limbata. Lw.

140.Py c no m a 11 a. Gerst. - Stratiomys. F. splendens, F.

141. Alliocera. Saund. - Stratiomys. Egg. graeca. Saund. clavicornis. Egg.

142. Stratiomys. Geoffr. - Musca. L. - Tabanus. Frisch. chamaeleon. I. nigrodentata. $\mathrm{Mg}$. ol. aquatica. Frisch. sellata. Sulzer. cenisia. Mg: potamida. $\mathrm{Mg}$.

chamacleon. Mg. ol. concinna. Mg. equestris. $\mathbf{M g}$. erythrocera. Egg. flaviventris. LTw. furcata. $\mathrm{F}$. panthaleon. Fll. longicornis. Scop.

strigata. F. thoracica. F. villosa. Mg. ol. nubeculosa. Mg. ol. riparia. $\mathrm{Mg}$.

strigata. Mg. ol.

hirtuosa. $\mathbf{M g}$. tomentosa. Schrk. anilis. Sehrk.

russica, Gimmerth. 143. Odon tomyia. Mg. - Musca.L. - Stratiomys. Auct.

tigrina. F. nigrita. Fll. flavissima. Rossi. decora. Mg. semiviolacea. Brullé. infuscata. Mg. ornata. $\mathrm{Mg}$. furcata. Mcq. annulata. Mg. septemguttata. Mg. felina. $\mathrm{Pz}$. vulpina. Germ. hydropota. Wlk. marginata. $\mathrm{E}$. personata. Lw. viridula, F. dentata. Mg. canina. Mg. subvittata. Mg. jejuna. Pz. bimaculata. Mg. interrupta, Lw. hydroleon. L. angulata. $\mathrm{Mg}$. ol. angulata. $\mathrm{Pz}$.

brevicornis, Lw, ol. ruficornis. Ztt. hydropota. Mcq. 
hydrodromia. Mg:

hydropota. Mg.

hydrophila. Lw.

argentea. F.

paludosa. Schum.

microleon. L.

latifasciata. Mcq.

limbata. Mg.

halterata. Schrk.

lunata. Enc. méth.

$$
\text { Sarginae. }
$$

144. Exochostoma. Meq.

nitida. Meq.

145. Sargus. F. $-\mathrm{Ne}-$ motelus. Deg. -

Musca. L.

bipunctatus. Scop.

Reaumuri. F.

infuscatus. MIg.

auratus. Mg. ol.

cuprarius. Fll.

iridatus. Wlk.

cuprarius. L.

violaceus. Scop.

nubeculosus. Ztt.

flavipes. Mg.

angustifrons. Lw.

albibarbus. Lw.

frontalis. $L w$.

nitidus. $\mathrm{Mg}$.

minimus. Ztt.

nitidus. Ztt. ol.

nigripes. Ztt.

rufipes. Whlbg.

sulphureus. Mg.

146. Chry s om yia. Meq.

- Musca. L. - Ne-

motelus. Deg: Chrysomyia u. Microchrysa. Lw. Chloromyia. Dunc.Clorisoma. Rond. formosa. Scop.

aurata. F. xanthoptera. E. flavogeniculata. Deg. azurea. Lw.

melampogon. Zil. speciosa. Meq. flavicornis. Mg. pallipes. Mg. cyaneiventris. Ztt. polita. L.

cyanea. F. splendens. Mg. ol. aurata. Deg.

\section{Berinae.}

147. Acanthomyia. Schin. - Beris. Ztt. dubia. Ztt.

148. Beris. Ltr. Arusca. L. Först. Stratiomys. F. Xylophagus. Ltr. clavipes. $\mathrm{L}$. nigra. Geoffr. chalybeata. Först. sexdentata. Zitt. flavipes. Mcq. obscura. Ztt. atra. $\mathrm{Mg}$. vailata. Först. nigritarsis. Ltr. clavipes. Mg. ol. fuscipes. Mg. nigra. Mg. geniculata. Curtis. Morrisii. Dale. pallipes. Lw.

149. Actina. Mg. Stratiomys. Gmel. Xylophagus. Ltr. Beris. Auct. nitens. Ltr.

hirsuta. Meq. similis. Först. nigripes. $\mathrm{Mg}$. chalybea. Mg. femoralis. $\mathbf{M g}$. Havofemorata. Mg. tibialis. $\mathbf{M g}$.

\section{Xylophag Idae.}

150. Subula. Mg. Xylophagus. F. Ltr. maculata. $\mathrm{F}$. marginata. Mg. varia. Mg. maculata. Ltr. atra. Ltr. citripes. L. Duf. 151. Xylophagus. Mg. - Nemotelus. Deg. ater. F. cinctus. Deg. 152. Pachy s tom us. Ltr. - Rhagio u. Empis. Pz. syrphoides. Pz.

XII. Coenomy Id ac. 153. Coenomyia. Lti. - Tabanus. F. Gmel.

- Sicus. F. M. Stratiomys. Pz. Musca. Scop. ferruginea. Scop. errans, $\mathbf{F}$. bicolor. F. unguiculata. Pz. bidentata. F. bispinosa. $\mathbf{F}$. macroleon. $\mathrm{Pz}$. grandis. Schrk. major. Schrk. palatina. Schrk. 
XIII. 'I' a b a $แ$ I d a c.

Tabaninae.

154. Ha emat o p o ta. Mgg.

- Tabanus. L

pluvialis. L.

hyetomantis. Schrk.

equorum. $\mathrm{F}$.

crassicomis. Whlbg. italica. Míg.

elongata. Enc. méth.

longicoinis. Mer.

tenuicornis. Meq.

grandis. Meq.

gymnonota. Brullé.

variegata. F.

Insitanica. Guér.

155. Hexato ma. Mg. -

Tabanus. F. - Hep-

tatoma. Mg. ol.

pellucens. F.

allipes, Schrk.

bimaculata. F. Mg.

156. T a ba 11 us. L.

micans. Mg.

austriacus. $\mathrm{F}$.

signatus. $\mathbf{M g}$.

auripilus. $\mathrm{Mg}$.

lugubris. Ztt.

nigerrimus. Ztt.

aterrimus. $\mathrm{Mg}$.

aethiops. Ljungh.

gigas. Hbst.

albipes. $\mathrm{F}$.

ignotus. Rossi.

tricolor. Zllir.

acuminatus. Lwv.

anthophilus. Lw.

tarandinus. L.

borealis. F.

lateralis. $\mathrm{Mg}$.

solstitialis. Mg.

tropicus. I,w.
Luridus. Fll.

tropicus. Scop.

tropicus. I.

luvidus. Lw.

paganus. F.

plebejus. Fll.

quadrinotatus. Mg. .

nigricormis. Ztt.

alpinus. Ztt.

pilosus. Lw.

bifarius. Lw

rusticus. L.

italicus. $\mathrm{F}$.

fulvus. Mg.

alpinus. Curtis.

rusticus. Schrk.

ferus. Schrk.

montanus. Mg.

ruralis. Ztt.

tergestinus. Egg.

spodopterus. $\mathbf{M g}$.

sudeticus. Zllr.

intermedius. Egg.

bovinus. I.

fulficornis. $\mathbf{M g}$.

ferrugineus. $\mathrm{Mg}$.

infusus. Wlk.

infuscatus. Lw.

graecus. F.

glaucopis. Mg.

lunulatus. Mg.

flavicans. Zllr.

cognatus. Lw.

cordiger. $\mathrm{Mg}$.

atricornis. MIg.

laticornis. Ztt.

vicinus. Egg:

unifasciatus. Lw.

autumnalis. L.

spectabilis. Lw.

maculicornis. Ztt.

nigricans. Egg. bromius. L.

maculatus. $\mathrm{Deg}$.

glaucescens. Schin.

glaucus. $\mathrm{Mg}$.

scalaris. Mg.

nemoralis, Mg.

umbrinus. Mg.

istriensis. Mg.

maculipennis. Brullé.

ater. Rossi.

morio. F.

fuscatus. Mcq.

obscurus. Lw.

alexandrinus. W.

curbonarius. $\mathrm{Mg}$.

nigrita. $\mathrm{E}$.

anthracinus. $\mathrm{Mg}$.

vittatus. F.

sublunaticornis. Ztt.

flaviceps. Ztt.

albomaculatus. Ztt.

confinis. Ztt.

punctifrons. Whlbg. lapponicus. Whlbg. maroccanus. F.

taurinus. $\mathrm{Mg}$.

lumatus. F.

carbonatus. Meq.

rufipes. $\mathbf{M g}$.

apricus. $\mathbf{M g}$.

macularis. $\mathrm{F}$.

dimidiatus. $\mathbf{M g}$.

chlorophthalmus. Mg.

bimaculatus. Meq. segmentarius. Brullé. lateralis. Brullé.

decisus. TV. morio. I.

Pangoninae.

157. Silvius. Mg. Tabanus. I. 
vituli, F.

hirtus. Lwv. algirus. Mg.

158. Nemorius. Rond. Chrysops. Mg. singularis. Mg. vitripenuis, $\mathrm{Mg}$.

13̈9. Chrysops. Mg. Tabanus. L. marmoratus. Rossi. flavipes. Mg. italicus. Lw. nigriventris. Lw. salinarius. Scop. fenestratus. Ltr. perspicillaris. Lw. rufipes. Mg. sepulcralis. F. quadratus. Mg. pictus. $\mathrm{Mg}$. viduatus. $\mathrm{Mg}$. caecutiens. L.

lugubris. L. viduatus. F. relictus. Mg. viduatus. Fll.

caecutiens. $\mathrm{Pz}$. parallelogrammus. Zllr. italicus. $\mathrm{Mg}$. fenestratus. F. nigripes. Ztt. melanopleurus. Whlbg. lapponicus. Lw. concavus. Lw. connexus. Lw. punctifer. Lw.

160. Pangonia. Ltr. Tabanus. L. - Bombylius. Oliv. - Tanyglossa. Mg. ol. maculata. Rossi. proboscidea. F. tabaniformis. Ltr. varipennis. Ltr. haustellata. Olio. variegata. Mcq. fumida. Lw. ferruginea. Mg. affinis. $\mathrm{Lw}$. marginata. F. haustellatc. F. mauritana. I. cellulata. Brullé. micans. Mg. ornata. Mg. griseipennis. Lw. dimidiata. Lw. aperta. Lw. variegata. $\mathrm{F}$.

maculata. Mg. picta. Meq. flaya. Mg.

\section{b. Orthocera.}

\section{XIY. Nemestrinidae.}

161. Nemestrina. Ltr. aegyptiaca. WV. Perezii. L. Duf.

162. Hirmoneura. Mg. obscura. $\mathrm{Mg}$.

163. Fallenia. Mg. Cytherea. F. fasciata. F.

164. Rhynchocepha$1 \mathrm{us}$. Fischer. - Volucella. Pall. - Fallenia. Mg. - Nemestivina. IV.

caucasica. W.

'Tauscheri. Fischer. taurica. IV.
XV. B o m b y lidae.

165. Anthrax. Scop.Musca. L. - Nemotelus. Deg. - Bibio. Rossi.

Paniscus. Rossi. modesta. $\mathrm{Mg}$. cingulata. Mg.

Hava. Mg. holtentota. Mg. ol. hottentota. L. circumilata. $\mathrm{Mg}$. leucostoma. $\mathrm{Mg}$. margaritifera. L. Duf. marginalis. Mg: venusta. $\mathrm{Mg}$. cana. Mg. cingulum. Mg: concinna. $\mathrm{Mg}$. Ablaclon. $\mathrm{F}$. distincta. $\mathrm{Mg}$. scutellata. Mfg. bimaculata. Meq. humilis. Ruthe. mucida. Zllr.

Ixion. F.

quinquefasciata. $\mathrm{Mg}$. elegans. WV. obliterata. Lw. afra. F.

fimbriata. Mg. vagaus. Lw. muscaria. W. Polyphemus. Mg* fellestrata. Fll. morio. L. pt. maura. Mg. ol. ornata. Curtis. variegat $a$. W. maura. L.

Daemon, Pz. bifasciata. $\mathrm{Mg}$. 
morio. L.

semiatra. Mg.

relutina. Mg.

holosericea. Mg. ol.

bicincta. Mg.

nycthemera. Mg.

occulta. Mg.

punctata. WT.

punctulata. Mg.

punctata. Mcq.

scrutata. Mg.

formosa. L. Duf.

obscura. Web.

punctum. Lw.

166. Exo prosopa. Meq.

- Mresca. Gmel. -

Bibio. F. - Anthrax.

Auctor.

stupida. MIg.

Rhadamanthus. Mg. rutila. W.

Miegii. L. Duf.

Aencus. Mg.

livida. IV'.

lutea. Meq.

bombyciformis. L.Duf. grandis. WV.

fasciata. L. Duf.

rhymnica. Eversm.

vespertilio. IV.

Megerlei. Mg.

campicola. Eversm.

picta. Mg.

Pandora. F.

Jochus. F.

Jachus. F.

Megaera. MIg.

italica. $\mathrm{Mg}$.

Cleomene. Egg.

capucina. F.

calopter $\alpha$.
Germari. WV.

Minos. Mg.

albiventris. Mcq.

phaeoptera. Mg.

rivularis, Mg.

sabaeus. Mg. ol.

argyrocephala. Mcq.

hilaris. Eversm.

melanoptera. W.

algira, F.

singularis. Mcq.

sicuía. Mcq.

Pygmaleon. F.

Daubei. Guér.

nebulosa. I. Duf.

interrupta. Muls.

squamea. Muls.

pectoralis. Lw.

167. Argyromoeba.

Schin. - Musca. L.

- Bibio. Rossi. E. -

Anthrax. Auct.

etrusca. F.

Satyrus. Rossi.

ruliginipennis. Meq.

tripunctata. $\mathrm{Mg}$.

difficilis. Mg.

Isis. Mg.

binotata. $\mathrm{Mg}$.

subnotata. Mg.

leucogaster. Mg.

aethiops. F.

punctata. Mg.

varia. $F$.

virgo. Egg.

sinuata. Fll.

morio. L. pt.

anthrax. Schrk.

Hesperus. Mg.

trifasciata. Mg.

trinotata. I. Duf.

capitulata. Muls.

relox $\mathrm{I} w$.
168. Mulio. Ltr. - Cytherea. F. - Glossista. Rond. pt. obscurus. F. infuscatus. $\mathbf{M g}$. punctipennis. Mcq. aureus. F.

brevirostris. Enc. meth . 169. Chalcoelit on. Lw.

- Mulio. Auct. Anthrax. F. Meq. Cyllenia. Wlk.

holosericeus. F. aberrans. Wlk.

semiargentea. Mcq.

Pallasii. Lw.

holosericeus. W.

speciosus. L.

170. Lomatia. Mg. -

Musca. Gmel. - Bi-

bio. E. - Anthvax.

F. Pz. - Stygia. Mg. ol.

Belzebul. F.

Lachesis. Egg.

Sabaea. F.

Atropos. Egg.

lateralis. $\mathrm{Mg}$.

Belzebul. $\mathrm{Pz}$.

Hecate. Meq.

171. Bombylius. L. Asilus. Gffr.

analis. F.

discoideus. F.

punctatus. F.

ambustis. W.

dispar. $\mathrm{Mg}$.

fuscus. F.

lugubris. $\mathrm{Lw}$.

barbula. WV.

ater. Scop.

pictus. P'z.

planiconis. $\mathrm{F}$. 
discolor. MIg. medius. Scop.

concolor. Zllr.

punctipennis. Lw.

medins. L.

discolor. Meq. concolor. Mik. punctatus. Deg. pictipennis. Lw. pallipes. Lw. major. L.

variegatus. Deg. sinuatus. Mik. consanguineus. Mcq. basilinea. Lw. fimbriatus. Mg. dimidiatus. $\mathrm{Mg}$. ventralis. Lw. torquatus. Lw. undatus. $\mathrm{Mg}$. undatus. Mik. diagonalis. $\mathrm{Mg}$. renosus. Mik. minor. Zllr. holosericeus. Mg. canescens. Mik. variabilis. Lw. pallens. Mg. fuliginosus. W. brevirostris. $\mathrm{Mg}$. nubilus. Mik. pilirostris. Lw. senex. Mg. deses. $\mathbf{M g}$. cruciatus. F.

leucopoyon. $\mathrm{Mg}$. ol. posticus. F. analis. Oliv. quadrifarius. Lw. fugax. W. vulpinus. Mg. posticus. Mg: micans. $\mathrm{Mg}$. cinerascens. Mik. favillaceus. Mg. fulvescens. Mg. longirostris. $\mathrm{Mg}$. apicalis. Mg. pumilus. Mg. citrinus. Lw. minor. L.

dilutus. Mg. subcinctus. Mg. cinerarius. W. niveus. W.

discoideus. Mg. ol. trichurus. W. cinereus. Mg. floralis. Mg. lusitanicus. Mg. pusillus. Mg. minimus. $\mathrm{Mg}$. pusio. Mg. ragabundus. $\mathbf{M g}$. semifuscus, Mg. axillaris. $\mathrm{Mg}$. obliquus. Brullé. pallidicrus. Brullé. nigripes. Mcq. nanus. Mg.

pygmaeus. Heq. albibarbis. Ztt. pumilus. Ztt. fumosus. L. Duf. vertebralis. L. Duf. minor. Mg. mus. Bigot.

172. Systoechus. Lw. - Bombylius. Auct. nitidulus. F. diadema. Mg. caudatus. $\mathrm{Mg}$. hyrcanus. W. stramineus. $\mathrm{Hg}$. setosus. Lw. sulphureus. Mik.

flavus. $\mathrm{Mg}$. minimus. F. fulvus. Mg. ctenopterus. Wlk. ctenopterus. Mik. aurulentus. Mg. leucophaeus. Mg. lucidus. Lw. exalbidus. $\mathbf{M g}$. gradatus. Mg. microcephalus. Lw. sericeus. Mg.

nubilus. $\mathrm{Mg}$. autumnalis. W.

173. Dischistus. Lw。 - Bombylius. Auct. minimus. Schrk. sulphureus. F. barbula. Lw. breviuseulus. Lw. unicolor. Lw. nigriceps. Lw. 174. Ploas. Ltr. - Bombylius. F. - Conophorus. Mg. ol. virescens. F. maurus. Mik. hirticornis. Ltr. lurida. Mg. grisea. F.

atrata. Lw. fuliginosa. $\mathrm{Mg}$. flarescens. Mg. fuscipennis. Meq. macroglossa. L. Duf. fuminervis. L. Duf. bivittata. Lw. 175. Cyllenia. Ltr. Asilus. Rossi. maculata. Ltr. rustica. Rossi. 
176. Eelimus. Lw。 gracilis. Lw. perspicillaris. Lw.

177. Phthiria. Mg. -

Volucella.F. - Bombylius. Mik.

Gaedii. Mg. maculata. $\mathrm{Mg}$. punctata. Mg. pulicaria. Mik.

pygmaea. F. campestris. Fll. nigra. $\mathrm{Mg}$. ol. scutellaris. Mg. umbripennis. Lw. fulva. Ltr. collvergens. Lw. canescens. Lw. vagans. Bigot. umbripenuis. Bigot. vagans. Lw. minuta. F. notata. Bigot.

178. Amictus. W. Thlipsomyza. W. variegatus. $\mathrm{Mg}$. 179. Eniconeura. MIeq. fuscipennis. Meq. 180. Oligodranes. Lw. fumipennis. Lw. obscuripennis. Lw.

181. Geron. Mg. - Bombylius. Mg. ol. gibbosus. Mg. hybridus. Hg. ol. halteralis. Mg.

182. Toxophora. Mg. - Bombylius. F.

maculata. W.

183. Usia. Ltr. - Volucella. F. - Bombylius. Rossi. aurata. $\mathrm{F}$.

aenea. $\mathrm{Mg}$. florea. Mg. atrata. E. florea. $\mathrm{F}$. vicina. Meq. forcipata. Brullé. incisa. W.

major. $\mathrm{Meq}$. grata. Lw. manca. Lw. versicolor. $\mathrm{F}$. hyalipennis. Mcq. ? pusilla. Mg. 184. Cyrtosia. Perris. marginata. Perris. nitens. Lw. obscuripes. Lw. opaca. Lw.

XVI. A croceridac.

183. Sphaerogaster. Ztt. - Platygaster. Ztt. ol.

arcticus. Ztt.

186. Ogeodes. Ltr. Musca. L. - Nemotelus. Schätfo - Syrphus. Pz. - Henops. F. $\mathrm{Mg}$. gibbosus. L.

leucomelas. Fll. Mg. cingulatus. Erichs. apicalis. Mg. zonatus. Erichs. giblosus. Ltr. Pz. pallipes. Ltr. marginatus. Mg. gibbosus. Mg. ol. Fll. Farius, Ltr.

limbatus. $\mathrm{Mg}$. fuliginosus. Erichs. fumatus. Erichs. pubescens. Ltr. nigripes. Ztt.

187. A crocera. Mg. Syrphis. F. Pz. Henops. Fll. globulus. Pz. orbiculus. F. albipes. Mg. tumida. Erichs. sanguimea. Ltr. nigrofemorata. $\mathrm{Mg}$. trigramma. Lw. laeta. Gerst.

188. P ter od on tia. Griff. - Henops. Klug. Astomella. Mg.

Waxelii. Klug. 189. Astomella. Ltr. curviventris. L. Duf. maryinata. Mg. clavicornis. Ltr. aurea. Erichs. Lindenii. Erichs. Waxelii. v. d. Lind. Vanetii. Costa. 190. Cyrtus. Ltr. Syrphus. F. - Empis. Vill, - Acrocera. F. Mg. ol. gibbus. F. acephalus. Ltr. pusillus. Meq. 191. Opsebius. Costa. - Pithogaster. Lw. inflatus. Lw. perspicillatus. Costa.

XVII. Scenopinidae. 192. Scenopinus. Ltr. - Musca. L. - Nemotelus. Pz. - Atrichia. Schrk. 
f'enestraliș. L. saltitans. Scop. spoliatus. Scop. senilis. F. sulcicollis. Mg. vitripennis. $\mathrm{Mg}$. domesticus. Mg. rufitarsis. $\mathrm{Mg}$. furcinervis. Ztt. niger. Deg. tarsatus. $\mathrm{Pz}$. ater. Fll. rugosus. $\mathrm{F}$. fenostialis. F. nigripes. $\mathrm{Mg}$. fasciatus. Wlk. glabrifrons. Mg. halteratus. Mg. orbita. Mg.

laevifrons. Lw. graminicola. $\mathrm{Ztt}$. Zelleri. Lw.

XVIII. Therevidae.

193. Xestomyza. Tipula u. Hirtea. E. - Ploas. L. Duf. Cionophora. Egg. Baryphora. Lw. chrysanthemi. F. rhayioniformis. L. Duf. calyciformis. L. Duf. Kollari. Egg. speciosa. Lw.

194. Thereva. Ltr. Musca. L. - Tabanus. Gffr. - Nemotelus. Deg. - Bibio. F. - Dialineura. Rond. pt. - Psilocephala. Ztt. pt.
Thereva. so str.

praecox. Egg.

nobilitata. F. nobilis. Gmel.

hirta. Deg. plebeja. Fll. oculata. Egg. fulva. Mg. subfasciata. Schum. annulata. F. anilis. F. ol. lunulata. Ztt. ursina. Whlbg. plebeja. L. strigata. F. fasciata. Deg. rustica. Fll. lugens. Lw. circumscripta. Lw. frontalis. Schum. marginula. Mg. albipennis. Ztt. fuscinervis. Ztt. apicalis. W. bivittata. Lw. Iugubris, F. arcuata. Lw.

taeniata. $\mathrm{Mg}$. tristis. Lw. subtilis. Lw. tuberculata. Lw. spinulosa. Lw. binotata. Lw. bipunctata. Mg. nervosa. Lw. poeciloptera. Lw. caesia. Mg.

Dialineura. Rond. auricincta. Egg. anilis. L. flavipes. F. sordida. Pr. fuscipennis. Mg. alpina. Egg. superba. Egg. nigripes. Lw. rustica. Lw. ol. obtecta. Lw. valida. Lw. microcephala, Lw. brevicornis. Lw. atripes. Lw.

Psilocephala. Ztt. ardea. F. rustica. Pz. confinis. Fll. ruficaudis. Mg. eximia. Mg. nigripennis. Ruthe. lapponica. Ztt. melaleuca. Lw. dispar. Mg. imberbis. Fll. laticornis. Lw.

punctipenuis. W. cincta. $\mathrm{Mg}$. albipennis. Mg. funebris. Mg. lugubris. Mg. albilabris. Mg. flavilabris. Mg. cinifera. Mg. albicans. Mcq. rufipes. Mcq. lanata. Ztt. vetula. $Z$ tt. ruficornis. Gimerth. 195. Exapata. Meq. anthracoides. Meq.

XIX. II id a s id a e. 196. Midas. W. lusitanicus. Mg. 
fulviventris. L. Duf. rufipes. Westw.

XX. Is illidae.

Loptogastrinae.

197. Leptogaster. Mg. - Asilus. Deg. F.

- Dasypogon. $\mathrm{k}$. Gonypes. Ltr. Mcq. cylindricus. Deg.

lividus. Gftr.

tipuloides. $\mathrm{F}$.

fuscus. Mg.

guttiventris. Ztt.

cylindricus. Mg.

pallipes. $\mathrm{v}$. Ros.

pedunculatus. Lw.

dorsalis. Dahlb.

nigricornis. Lw.

pubicornis. Lw.

subtilis. Lw.

variegatus. Lw.

nitidus. Meq.

pumilus. Meq.

Palasii. IV.

hispanicus. Mg.

\section{Dasypogoninae.}

198. Dioctria. Mg. Asilus. L.

rufithorax. Lw.

humeralis. Zllr.

longicornis, Mg.

lateralis. MIg.ol.

melanopogon. Egg.

calceata. Mg.

Reinhardi. Mg.

cothurnata Mg.

umbellatas'um. Mg.

Meigenii. Schrk.

lata. Lw. atricapilla. $\mathrm{Mg}$.

atrata. $\mathrm{Mg}$

nigripes. Mg.

fuscipennis. Fll.

Fallenii. Mg.

fuscipes. Meq.

aurifrons. Mg.

flavipennis. Mg.

gagates. $\mathbf{M g}$.

semihyalina. Mg.

Hercyniae. Lw.

bicincta. Mg.

annulata. $\mathrm{Mg}$.

infuscata. Mg.

oelandica. L.

rufipes. Deg.

frontalis. $\mathbf{E}$.

hyalipennis. F.

gracilis. MIg.

flavipes. Mg. varipes. Mg.

frontalis. Mg.

rufipes. Zllir.

Baumhaueri. Mg.

linearis. F.

laeta. Lw.

lateralis. Mg.

haemorhoidalis. Mg. speculifrons. Mg.

Wiedemanni. MIg.

caesia. IV.

maculata. W.

199. A pogon. Perris.

Dufouri. Perris.

200. Das y pogon. Mg.

- Asilus. L. - Erax.

Scop. - Cheilopogon.

Rond. pt.

teutonus. L.

tenthredoides. Scop. diadema. $\mathbf{F}$.

puntatus. $\mathrm{F}$.

nervosus. $\mathrm{Pz}$.

liburnicus. $\mathrm{Mg}$.

variabitis. Brullé.

cylindricus. F.

fasciatus. Mg.

analis. $\mathrm{F}$.

bohemicus. Preyssl.

atratus. $\mathrm{F}$.

nubilus. Mg.

Fabricii. Mg.

arcuatus. $\mathrm{E}$.

fimbriatus. Mg.

ruficauda. F.

Waltlii. Mg.

Kolenatii. Gimmerth.

rufipes. Gimmerth.

nigriventris. I. Duf.

201. Saropogon. Lw. - Dasypogon. Auctor. - Laphria. MIg. pt. - Dioctria. Costa.

luctuosus. Mg. nigripennis. Costa.

leucocephalus. Mg.

scutellaris. Mg.

flavicinctus. Mg.

axillaris. Lw.

aberrans. Lw.

202. Xiphocerus. Meq.

Lw. - Asilus. Rssi.

F. - Laphria. F.

Dasypogon. W. Mg.

- Elasmocera. Rond.

glaucius. Rossi.

variegatus. W.

limbatus. F.

senes. L. Duf.

203. Ha bropogon. Lw.

- Dasypogon. $\mathrm{F}$. 
longiventris. Lw exquisitus. Mg. vutilus. $\mathrm{Mg}$. striatus. F.

favillaceus. Lw. 204. S t елор о gо и. Lw. - Asilus. F. - Dasypogon. Auct. coracinus. Lw. callosus. W. sabaudus, F. tristis. Mg. elongatus. MIg. fulvus. $\mathrm{Mg}$. tanygaster. Lw. ochreatus. Lw. xanthotrichus. Brullé. graecus. Lw. pyrhous. TV. denudatus. Lw. brevipennis, Mg. junceus. Mg. strataegus. Gerst. schisticolor. Gerst.

205. S tich op og o n. Lw. - Dasypogon. Auct. albofasciatus. $\mathrm{Mg}$. nigrifrons. Lw. elegantulus. Mg.

tener. Lw.

Frauenfeldi. Egg. barbistrellus. Lw. inaequalis. Lw.

elegantulus. Mg. pt. scaliger. Lw. ripicola. L. Duf. 206. Holopogon. Lw.

- Dasypoion. Auct.

- Eriopogon. Lw. pt. brunnipes. $\mathrm{Mg}$. clavipes. Lw. priscus. MIg. auribarbis. $\mathrm{Mg}$. nigripennis. Mg. fumipennis. Mg. albipilus. Mg: venustus. Rssi. minutus. F. Iris. $\mathrm{Mg}$. siculus. Meq. timidus. Lw. dimidiatus. $\mathrm{Mg}$. fuscipennis. Mg. chalcogaster, L. Duf. melas. L. Duf. claripenuis. Lw. melaleucus. Mg. laniger. Lw.

207. Oligopogon. Lw. hybotinus. Lw.

208. Is o p og on. Lw. Dasypogon. Auct. Dioctria. Mg. ol. Leptarthrus. Steph. brevirostris." $\mathbf{M g}$.

longitarsis, Fll. armillatus. Fll. apicalis. $\mathrm{V}$. Ros. hottentotus. F. hyalipennis. Mg. ol. vitripennis. $\mathrm{Mg}$. 209. He te r o pogon. Lw. - Dasypogon. Mg. manicatus. Mg. ornatipes. $L w$.

210. Triclis. Lw. Gastrichelius. Rond. olivaceus. Lw.

211. Lasiopogon. Lw.216. Laphystia. Lw. - Asilus. F. - Da- sabulicola. Lw.

sypogon. Auct. cinctus. $\mathbf{F}$.

hirtellus. Fll.

cinctellus, Mg. Macquarti. Perris. pilosellus. Lw. hirtellus. Mg.

212. Cyrtopogon. Lw. - Asilus. F. - Dasypoyon. Auct. Dioctria L. Duf. lateralis. Fll. flarimanus. $\mathrm{Mg}$. maculipemnis. Mcq. flavimanus. Mg. pt. litura. Zllr. monticola. Schum. ruficornis $\mathrm{E}$. fulvicornis. Meq. interruptus. $\mathrm{Mg}$. ochraceus. L. Duf. tenuibarbus. Lw. luteicornis. Ztt. lapponicus. Ztt. centralis. Lw. quadrizonatus. Lw. longibarbus. Lw.

213. A cne phalum. Meq. Olivierii. Mcq.

214. Pycnopogon. Lw. - Laphria. Brullé. L. Duf. apiformis. Lw. pallidipemnis. Brullé. fasciculatus. Lw. laniger. L. Duf. 215. Aparolius. Lw. jubatts. Lw.

\section{Laphrinae.}

17. Laphria. Mg. Asilus. L. - Erax. Scop. - Pogonosoma. Rd. pt. maroceana. $\mathrm{E}$. anthrax. Mg. 
ephippium. $\mathrm{F}$. dorsalis. Deg.

flava. L.

ephippium. Pz. pedemontana. F. gibbosa. L. bombytius. Deg. gilva. L.

mufa. Deg. ignea. Mg. gilva. Mg. ol. fuliginosa. $\mathrm{Pz}$. cincta. Mg. auribarbis. $\mathrm{Mg}$. aurea. $\mathrm{E}$.

lutea. $\mathrm{Mg}$. vulpina. Mg. timbriata. Mg". fulva. $\mathrm{Mg}$. proboscidea. Lw. aurifera. L. Duf. marginata. L. niyra. Mg. fulgida. Mg. femorata. $\mathrm{Mg}$. dioctriaeformis. $M \mathrm{~g}$. tibialis. $M g$. rutipes. FH. lapponica. Ztt. jufipes. Ztt. ol. nigripemmis. Mg. dizonias. Lw. limbata. Mcq. venatrix. Lw. ursula. Lw. Hecate. Gerst. auriflua. Gerst. empyrea. Gerst. meridionalis. Muls. nitidula. F. chrysocephala. Mg". breripemis. $\mathbf{M g}$.
Havescelis. Heq.

coarctata. L. Duf.

218. Andrenosoma. Rond. - Asilus. L. - Laphria. Auct.

albibarbis. $\mathrm{Mg}$. erythrura. Lw. atra. J.

violacea. Mg. ol. violacea. $\mathrm{F}$.

Asilinae.

219. Promachus. Lw. - Asilus. F. Mg. Trupanea. Meq. pictus. $\mathrm{Mg}$. maculatus. F. leoninus. $\mathrm{Lw}$. 220. Polyp houi us. Lw. laevigatus. Lw. 221. Asilus. L. Lophonotus. Meq. Lw. - Protophanes u. Dismachus. $\mathrm{Lw}$. cristatus. Mg* punctipennis. $\mathbf{M g}$. punctatus. Mg. ol. spinig*er. Zllr. fuscipennis. $\mathrm{Mg}$. forcipula. Zllr. mixtus. Lw. forcipatus. L. praemolsus. Lw. tridens. Ego'. bimucronatus. Lw. trigonus, Mg. albipilus. $\mathrm{Mg}$. pullus Mg. hispidus. Zllr. basalis. I ww. stylifer. Lw. hamulatus. Lw. bifurcus. Lip. picipes. $\mathbf{M g}$. varius. $\mathbf{M g}$. cochleatus. Lw. varians. Mg: tricuspis. ${ }^{\wedge} \mathbf{L w}$. crassicauda. Lw.

Eutolmus. Lw. rufibarbis. 1 IIg. melampodius. Zllx. apicatus. Lw. pictipes. Lw. Kiesenwetteri. Lw. periscelis. Lw. lusitanicus. Lw. sinuatus. Lw. decipiens. Mg. tephraeus. Mg. haematoscelis. Gerst. stratiotes. Gerst.

Machimus.Lw. cribratus. Lw. chrysitis. Mg. femoralis. Zllr. fortis. Lw. annulipes. Brullé. basalis, Lw. colubrinus. $\mathbf{M g}$. pilipes. $\mathbf{M g}$. fimbriatus. $\mathrm{Mg}$. gonatistes. Zllr. obscurus. Mg. rusticus. $\mathrm{Mg}$. genualis. Zllr. atricapillus. Fll. opacus. Mg". calceatus. Mg。 licornis. Zll. subulatus. L. rufimanus. $\mathbf{M g}$. plelejus. $\mathbf{M g}$. 
lacinulatus. Lw. dasypygus. Lw. setibarbus, Lw. lugens. Lw.

caliginosus. Mg. cyanopus. Lw. cerdo. Gerst.

Mochtherus. Lw.

brumnipes. F. castanipes. Mg. flavicornis. Ruthe.

Olivierii. Meq. llavipes. $\mathrm{Mg}$. Schineri. Egg. pallipes. $\mathrm{Mg}$. omissus. Mg. fulvipes. Mg. striatipes. Lw. malacias. Gerst. ochriventris. Lw.

Cerdistus. Lw. erythrurus. Mg. Zelleri. Schin. erythrurus. Lw. melanopus. Mg. alpinus. $\mathrm{Mg}$.

Stilpnogaster. Lw. aemulus. $\mathrm{Mg}$. stabitis. Zllr. nigricans. Meq.

It a mus. Lw. cyanurus. Lw. aestives. $\mathrm{Mg}$. tibialis. Fll. tabidus. Mg. niger. Deg. cothurnatus, Mg. geniculatus. Mg. impudicus. Gerst. dasymallus. Gerst.

Tolmerus. Lw. atripes. Lw. poecilogaster. Lw. pyragra. Zllr.

Epitriptus. Lw. cingulatus. F.

annulatus. $\mathrm{M} \mathrm{cq}$. setosulus. Zllr. nanus. Lw. stviatus. $\mathrm{Mg}$. senex. MIg. arthriticus. Zllr. emarginatus. Lw. culiciformis. W. inconstans. Mg. Antiphrisson. Lw. trifarius. Lw.

Asilus. Lw. crabroniformis. L. barbarus. L.

Rhadiuryus. Lw. rariabilis. Ztt.

Pamponerus. Lw。 germanicus, L. tibialis. F.

Antipalus. Lw.

varipes. Mg.

macrurus. Ruthe. ranthopygus. Ruthe. tenax. Z11r. aurifluus. Zllr.

Echthistus. Lw. rufinervis. W. flavescens. Mcq.
Philonicus.

albiceps. Mg. canescens. Mg. allibarbus. Zllr. nudus. Lw.

longitarsis. Mcq. tibialis. IV. canus. WV. cinerarius, IV. fuscidus. IV. gracilipes. Mg. lucidus. W. parvulus. Mg. micans. Mg. marginatus. $\mathrm{Mg}$. auripilus. $\mathrm{Mg}$. domitor. $\mathbf{N g}$. variegatus. Mg. versicolor. $\mathrm{Mg}$. siculus. Mcq. punctatus. Mcq. pumilus. Mcq. nigripes. Meq. naxius. Mcq. tessellatus. Brullé. melanotrichus. Brullé. tibialis. Gimmerth. intermedius. Ztt. glaucus. Ztt. setiventris. Ztt.

Xxí. Leptidac. 22.2. La m p r o my ia. Mcq. funebris. L. Duf. 223. Vermileo. Mcc. Musca. Deg. Gm. - Nemotelets. Deg.

- Rhajio. F. -

- Leptis. F. Mg.

Degeeri. Mcq. vermileo. Deg. 
224. Leptis. T. - Musca.

L. - Nemotelus. Dg.

- Rhagio. F. Pz.

fuscipennis. Mg. cingulata. Lw. scolopacea. L. strigosa. Mg. latipemis. Lw. maculata. Deg.

annulata. Mg. ol. nigrofasciata. Mg. ol. distigma. $\mathrm{Mg}$. immaculata. $\mathbf{M g}$.

stigmatica. Ztt, chrysostigma. Lw. vitripennis. Mg. stigma. Schum. striola. Mg. notata. $\mathrm{Mg}$.

Heyshami. Curtis. monticola. Egg. lineola. $\mathrm{F}$. allifrons. Mg. ol. tringaria. L. vanellus. $\mathrm{F}$. simplex. Mg. punctata. Lw. conspicua. IIg. annulata. Deg: conjungens. Ruthe. nigra. Mg. funebris. Mg. flavicornis. Meq: ephippium. Ztt. cinerea. Ztt. inutilis. WVIk. sordida. Lw. cinereofasciata. Schum. 225. Chry sopila. Mícq. - Rhagio u. Atherix. F. - Leptis. Mg. nubecula. Fll. nevelata. $\mathrm{F}$. bicolor. $\mathrm{F}$. auricollis. Mg. aurea. Mg. diadema. F. Mg. tipuliformis. Schrk. nigrita. F.

splendida. Mg. chlorophthalma. Lw. atrata. F.

cristata. F.

tomentosa. F. aurata. F. Mg. holosericea. WIk. cingulata. Don. erythrophthalma. Lw. luteola. Fll. helpola. Mg. flaveola. Mg. genius. P.. laeta. Ztt. tristis. Schum. 226. A therix. Mg. - 232. Hybos. Mg. - EmBibio, Rhagio u. Leptis. F.

Ibis. F.

Titanus. F. maculatus. Ltr. marginata. F. crassipes. Mg. flaripes. F. Mg. unicolor. Curtis.

227. Ptiolina. Staeg. Schin. - Atherix.Mg: - Leptis. Fll. melaena. $\mathbf{M g}$. pilosa. $\mathrm{Mg}$. crassicornis. Pz. griseola. Fll. immaculata. F. grisea. Mg. nebulosa. F. obseura. FH. nitida. Whlbg. nigripes. Ztt.

nigrina. Whlbg.

nigra. Ztt.

228. $\mathrm{S}$ p a 1 ia. $\mathrm{Mg}$.

nigra. Mg.

Fallenii. Hal.

229. Hilarimorpha. Schin.

singularis, Egg. tristis. Egg.

XXII. E mpida e.

Hybotinae.

230. B r a chy s to ma. $\mathbf{M g}$.

- Bacha u. Syrphus.

F.

resiculosa. $\mathrm{F}$. obscuripes. Lw.

131. Meghyperus. Lw. sudeticus. Lw. pis. F. - Asilus. F. - Musca. L. grossipes. L. clavipes. F. funebris. $\mathrm{F}$. culiciformis. Gmel. pilipes. Curtis. femoratus. Miill. flavipes. F. IIg. culiciformis, F. vitripennis. Mg. fumipennis. Mg. infuscatus. 7tt. rufitarsis. Ztt. pilipes. Mg. 233. Pterospilus. Rond. - Asilus, Stomoxys u. Dioctria. F. Hybos. Mg. - Syneches. Lw. muscarius. $\mathrm{E}$. 
234. Cyrtoma. Mg. Empis Fll. - Microsania. Ztt. pt. pallipes. Mg. nigra. Mg. spuria. Fll. atra. Mg. rufa. Mg. simplicipes. Ztt. sulcata. Ztt. melaena. Hal.

stigmaticalis. 'Ztt. pectipennis. Mg.

\section{Empinae.}

235. Rhamphomyia.

Mg. - Empis. F. platyptera. Pz. marginata. $\mathrm{F}$. latipennis. Mg. flava. Fll. spissirostris. Fll. nodipes. Fll. dissimilis. Ztt. paradoxa. Whlbg. filata. Ztt. albosegmentata. Ztt. aretica. Ztt. ol. laevipes. Fll. maculipennis. Ztt. morio. Ztt. modesta. Whlbg. simplex. Ztt. sulcata. Fll. canaliculata. Mcq. villosa. $Z \mathrm{tt}$. hirtula. Ztt. serpentata. Lw. erythrophthalma. Mg. alpina. Ztt. cillerascens. Mg. spinipes. Fll. rufipes. Ztt. dentipes. Ztt. tibialis. Mg. nigripes. $\mathbf{F}$. crassirostris. Fll. tephraea. MIg. atra. Mg. nitidula. Ztt. nitida. Ztt. ol. nitida. Meq. lugubrina. Ztt. fuscipennis. $\mathrm{Ztt}$. coracina. Ztt. confinis. Ztt. albipennis. Fll. niveipennis. $Z$ tt. lacteipemnis. Ztt. dispar. Ztt. fuliginella. Ztt. picipes. Ztt. unicolor. Ztt. culicina. Fll. rufipes. Mg. pt. tenuirostris, Fll. rufipes. Mg. pt. sciarina. Fll.

tipulariata. Ztt. hybotina. Ztt. tenuicomis. Ztt. tipularia. Fll. cinerea. $\mathrm{Mg}$. oedalina. Ztt. fratemella. Ztt. rariabilis. Fll. pennata. Mcq. obscura. Ztt. cana. Ztt. plumipes. Fll. geniculata. Mg. poplitea. Whilbg. plumifera. Ztt. tarsata. Mg. fascipennis. Ztt. obscurella. Ztt. costata. Zit. tibiella. Ztt. vesiculosa. Fll.

Fallenii. Mg. atripemnis. Ztt.

anthracina. Ztt. ol. alata. Ztt. vespertilio. $Z$ tt. aethiops. Ztt. gibba. Fll. alipes. $\mathbf{M g}$. anthracina. $\mathrm{Mg}$. alpestris. Schum. holosericea. $\mathrm{Mg}$. lucidula. Ztt. umbripennis. $\mathrm{Mg}$. nigripennis. Fll. obscuripennis. $\mathrm{Mg}$. anomalipemuis. Mg. infuscata. $\mathrm{Mg}$. anomalina. Ztt. hybrida. Ztt. rugicollis. Mg. longipes. Mg. bilineata. Mg. caesia. $\mathbf{M g}$. schistacea. Mg. carbonaria. Mg. ferruginea. $\mathrm{Mg}$. cinerea. $\mathrm{F}$. pilifer. $\mathbf{M g}$. appendiculata. Mcq. stigmosa. Mcq. Haviventris. Hcq. clypeata. Mcq. bicolor. Hícq. dispar. Curtis. saniculae. Curtis. hyalina. Brullé. amoena. Lw. gracilipes. Lw. obscura. Lw. 
squamigera. Lw.

flavicoxa. Scholtz. reflexa. Ztt.

flexicauda. Ztt. metatarsata. Ztt. fuscula. Ztt. glaucella. Ztt. pallidiventris. Fll. lividiventris. Ztt. caudata. Ztt. griseola. Ztt. trilineata: Ztt. ignobilis, Ztt. fumipennis. Ztt. lugens. Ztt. pusilla. Ztt. nigrita. Staeg. aperta. Ztt. 236. Empis. L. - Platyptera. Curtis. pt. meridionalis. $\mathrm{Mg}$. argyreata. Egg. maculata. F. variegata. $\mathrm{Mg}$. tessellata. $\mathrm{F}$

livida. F.

tipuloides. L. funebris. $\mathbf{M g}$. bistortae. Mg. ciliata. F. pennata. F. pennipes. Fll. boja. Schrk. plumipes. Ztt. fiumana. Egg. livida. L.

lineata. F. ignota. $\mathrm{Mg}$. punctata. MIg. dorsalis. Curtis. rustica. Fll. nigricans. $\mathrm{Mg}$. pteropoda. Egg. cognata. Egg. albicans, Ifg. gravipes. Lw. borealis. L. fallax. Egg. opaca. E'.

rufipes. F. vitripemnis. Mg. albinervis. Mg: hyalipennis. Fll. proxima. Mg. albinervis. $\mathrm{Mg}$. pusio. Egg. florisomma. Lw. chioptera. Fll. crassipes. Schrk. morosa. Mg. bievipennata. Meq. pennaria. Fll. albipemis. Mg. pemimes. Is. ciliata. Fll. pennetu. Selirk. longirostris. $\mathrm{Hg}$. ol. longirostris. Mg: vernalis. $\mathrm{Mg}$.

l. ucoptera. Wrik. decora. Mg. volucris. Mg: morosa. Mg. lutea. Mg. ochracea. Curtis. parvula. Egg. stercorea. L. mollogramma. Mg. punctata. F. digramma. $\mathrm{Mg}$. testacea. Ztt. testacea. F. scutellata. Curtis. trigramma. $\mathrm{Mg}$. discolor. Lw. ferruginea. $\mathrm{Hg}$.

morio. $\mathrm{F}$.

cothurnata. Brullé.

hispanica. Lw. trilineata. W. simplex. W. sulcipes. Mg. picipes. Mg. lepidopus. Mg. lineata. $\mathrm{Mg}$. grisea. $\mathrm{Mg}$. gravis. Mg. ardesiaca. Mg. truncata. Mg. fulvipes. $\mathrm{Mg}$. pilipes. Mg. brumipennis. Ifg. nigritarsis. Mg. nitidicollis. Curtis. crassipes. $\mathbf{M g}$. umbrina. $\mathrm{Mg}$. hyalinata. Mg. podagrica. Mg. elongata. Mg. funesta. $\mathrm{Mg}$. nuntia. Mg. turbida. MIg. rapida. Mg. rufiventris. Mg. ventralis. Ztt. modesta. Mg. stigma. Mg. fuscipes. Brullé. serricans. Brullé. unicolor. Brullé. atra. Meq. trivittata. Meq. obscura. Meq. subpennata. Mcq. brevipennis. Meq. gracilis. Curtis. lucida. Ztt. grisea. Fll, von $\mathbf{I I g}$. 
maculipes. Ztt. grisea. Fll. pt. geniculata. Ztt. cinerea. Ztt. furcata. Ztt. nitidula. Ztt. nigerrima. Lw。 rava. Lw.

haemi. Lw.

dispar. Scholtz. nigrescens. Schum. fasciata. Schum.

237. P a ch y m er i a. Steph. - Empis. Auct.

femorata. F. ruralis. $\mathrm{Mg}$. scotica. Curtis. nitida. MIg. palparis. Egg. quinquevittata. Mcq. tumida. $\mathrm{Mg}$.

238. Hormopeza. Ztt. obliterata. Ztt. 239. Ragas. Walk. unica. Wlk. 240. Gloma. Mg. fuscipennis. $\mathrm{Mg}$. 241. Or e oget oll. Schin. - Gloma. Lw.

basalis. Juw.

242. Hilara. Mg. -Bibio. Pz. - Tachydromia. F. - Empis. F. Fill.

matrona. Hal. spinipes. Ztt. cilipes. $\mathrm{Mg}$. spinimana. Ztt. infans. Ztt. pilipes. Ztt. flavipes. Mg. acephala. Pz. litorea. Fil. univittata. $\mathrm{Mg}$. nireipennis. Ztt. Sturmii. Mg. abdominalis. Ztt. gallica. Fll. canescens. Ztt. griseola. Ztt. nigritarsis. Ztt. fuscipes. $F$. plumbea. F. intermedia. Fll. pt. albida. $\mathrm{Mg}$. manicata. $\mathrm{Mg}$. Iurida. Fll. pruinosa. Mg. pinetorum. Ztt. clypeata. Mg. chorica. Fll. lugubris. Mg. nana. Meq. brevivittata. Mcq. longivittata. Ztt. nigrina. Fll. quadrivittata. Mg. intermedia. Fll. pt. bistriata. Ztt. vulnerata. Egg. lugubris. Ztt. maura. F. globuliceps. Mg. nitidula. Ztt. femorella. Ztt. hirtula. Ztt. pilosa. Ztt. senilis. Pz. interstincta. $\mathrm{Mg}$. interstincta. Fll. modesta. $\mathrm{Mg}$. aethiops. Ztt. tenella. Fll. flava. Schin. fasciata. $\mathbf{M g}$. trigramma, $\mathrm{Mg}$. infuscata. Brullé. sublineata. Brullé. cinerea, Mcq. fulvipes. Mcq. longirostris. $\mathbf{M c}$. rufipes. Mcq. obscuritarsis. Ztt. carbonella. Ztt. thoracica. Meq. ephippium. Scholtz. anomala. Lw. recedens. Hal. tenuinervis. Ztt. squalens. Ztt. obscura. Mg. minuta. Ztt. gracilipes. Boll. cingulata. Dahlb. 243. It eap hila. Ztt. Macquarti. Ztt. nitidula. Ztt.

Ocydrominae.

24. $\mathrm{Mi}$ i r o phorus. Mcq.

- Trichina. $\mathrm{Mg}$. -

- Oedalea. Ztt.pt. velutinus. Mcq. pusillus. Mcq. flavipes, Mg. pallipes. Ztt. ol. clavipes. $\mathrm{Mg}$. minuta. Ztt. crassipes. Meq. anomala. Mg. tarsella. Ztt. fuscipes. Ztt. rufipes. $\mathrm{Mg}$. elongatus. HaI. minutus. WVlk. 245. Oedalea. MIg. - Empis. Fll. Xiphidicere. Mcq. pt. 
hybotina. Fll. infuscata. Lw. minuta. Fll. flavipes. Ztt. tibialis. Meq. rufipes. Meq. Holmgreni. Ztt. pallipes. Ztt. stigmatella. Ztt. apicalis. Lw. tristis. Scholtz. pemnata. Gimmerth.

246. Ocydromia. $\mathrm{Mg}$. - Empis. Fll. glabricula. Fll. nigripennis. $\mathrm{Mg}$. scutellata. Mg. rufipes. Mg. dorsalis. Mg. melanopleura. Lw. nigripes. Ztt.

24\%. Leptopeza. Meq. - Ocydromia. Mg. flavipes. Mg. tibialis. Ztt. borealis. Ztt. flavimana. Ztt. nigripes. Ztt. ruficollis. $\mathrm{Mg}$. 248. Holoclera. Schin. pulchra. Egg.

249. Euthy n eura. Mcq. myrtilli. Meq. myricae. Wlk.

250. Anthalia. Ztt. -

? Euthyneura. Mcq. albipennis. Ztt. Gyllenhali. Ztt. nigra. Ztt. pallida. Ztt. rostrata. Ztt. cansobrina. Ztt.
Schoenherri. Ztt. immatura. Ztt.

Hemerodrominae.

25\%. Clino e ra. Mg. Lw.

- Empis. Fll.

Heleodromia. Hal. -

Hydradromia. Meq:-

Hemerodromia. $\mathrm{Mg}$.

pt. - Paramesia.

Meq. - Wiedemannia.

Ztt. - Brachystoma.

Ztt.

bistigma. Curtis.

borealis. Ztt.

juvenilis. Ztt.

lota. Wlk.

appendiculata. Ztt.

hygrobia. Lw.

bipunctata. Hal.

Zetterstedtii. WIk.

Zetterstedtii. Fll.

Bohemani. Ztt.

Robertii. Mcq.

stagnalis. Hal.

Westermanni. Ztt.

fontinalis. Hal.

Wesmaeli Ztt.

Wesmaeli. Mcq.

Escheri. Ztt.

aucta. Ztt.

tenella. Whlbg.

riparia. Rob.

inermis. Lw.

nudipes. Lw.

unicolor. Curtis.

nigra. $\mathrm{Mg}$.

232. Hemerodromia.

- Tackydromia. Fll. flavella. Ztt. melanocephala. Hal. stigmatica. Schin. monostigma. Wlk. praecatoria. Fll. monostigma. $\mathrm{Mg}$. raptoria. Mg. albicornis. Mg. raptoria. Fll. supplicatoria. Mg. oratoria. Fll. Frigelii. Ztt. trapezina. Ztt. unilineata. Ztt. oratoria. Mg. unicolor. Curtis. gracilipes, Lw. brevipes. Lw. lineata. Gimmerth.

253. Ardoptera. Meq. - Tachydromia. Fll. - Hemerodromia. Mg. - Leptosceles. Hal.

irrorata. Fll. guttata. Hal. exoleta. Hal. ocellata. Costa. oculata. Lw. anomala. Scholtz. nivalis. Ztt.

254. Trich o p e za. Rond. - Brachystoma. Mg. longicornis, $\mathrm{Mg}$.

255. Phyllo d romia.Ztt. - Tachydromia. F. Fll. - Hemerodromia. Mg. melanocephala. F. mantispa. $\mathrm{Mg}$. $\mathrm{Pz}$. o secratoria. Wlk. vocatoria. Fll. albiseta. Ztt. vocatoria. $\mathrm{Mg}$. 
256. Sciodromia. Hal. - Heleodromia. Mcq. pt. - Hemerodromia. Mg. - Microcera. Ztt. immaculata. Hal.? Mcq.

Tachydrominae.

25\%. Drapetis. Mg. Tachydromia. Fll. Hemerodromia. Heeger.—Stilpon. Lw.pt. lunata. W.

femorata. Heeg.

flavipes. Mcq.

exilis. Meq.

assimilis. Fll.

minima. Mg.

nigra. $\mathrm{Mg}$.

pygmaea. v. Ros.

exilis. Mg.

graminum. Fll.

celeripes. $\mathrm{Mg}$.

flexuosa. Lw. aenescens. W.

brunnipes. Meq.

nervosa, Lw.

setigera. Lw.

arcuata. Lw.

pusilla. Lw.

marginata. Mg.

aterrima. Curtis.

luteipes. Enc. meth.

pilipes. Lw.

nigripes. Ztt.

258. Platypalpus. Meq.

- Empis. F. Pz. -

Sicus. Ltr. - Tachydromia. Mg. Perris.

flavipes. $\mathrm{F}$.

vulgaris, Mg. ol.

agilis. $\mathrm{Mg}$.

curticornis. Ztt. macul ipes. Mg. cothurnatus. Meq. socculatus. Ztt. ol. ventralis. $\mathrm{Mg}$. major. Ztt.

flavicornis. Mg.

pallidiventris. $\mathrm{Mg}$. varius. Wlk.

infuscatus. $\mathrm{Mg}$.

bicolor. F.

flavipes. Fll.

cursitans. F.

calceatus. Mg.

articulatus, Meq.

dichrous. Mg.

ecalceatus. Ztt.

candicans. Fll.

fasciatus. Mg.

nigrinus. $\mathrm{Mg}$.

nigritellus. Ztt.

minutus. $\mathrm{Mg}$.

annulatus. Mg.

exiguus. $\mathrm{Mg}$.

annulipes. $\mathrm{Mg}$.

rufipes. $\mathrm{Mg}$.

notatus. Mg.

fascipes. Mg.

fulvipes. $\mathrm{Mg}$.

niger. Fll.

curvipes. $\mathrm{Mg}$.

femoralis. Ztt.

unguiculatus. Ztt.

castanipes. $\mathrm{Mg}^{\mathrm{g}}$.

vividus. $\mathrm{Mg}$.

ciliaris. Fll.

flavipalpis. Mg.

fuscicornis. Ztt.

pallipes. $\mathrm{Mg}$.

pallipes. Fll.

albocapillatus. Fll.

fuscitarsis. Ztt.

parvicornis. Ztt. comptus. Wlk.

stigmatellus. Ztt.

pallipes. Ztt.

nigritarsis. Fll.

pallidus. Mg.

exilis. $\mathrm{Mg}$.

luteus. Fll.

analis. $\mathbf{M g}$.

pectoralis. Fll.

gilvipes. $\mathbf{M g}$.

flavipes. Mg. ol.

stramineipes. Ztt.

collaris. $\mathbf{M g}$.

luteicornis. Mg.

cingulatus. Lw.

glabratus. Mg.

pygmaeus. Mg.

nigrifemoratus. Meq.

strigifrons. Ztt.

laticinctus. Wlk.

divisus. Wlk.

commiles. Wlk.

compungens. Wlk.

robustus. Wlk.

longiseta. Ztt.

coxatus. Ztt.

maculimanus. Ztt.

sordidus. Ztt

ater. Whlbg.

curvinervis. Ztt.

annulatus. Fll.

flavipalpus. Mcq.

dubius. Wlk.

rapidus. Mg.

pulicarius. Mg.

macula. Ztt.

flavipalpis. Ztt. pt.

brevicornis. Ztt.

fuscimanus. Ztt.

moriellus. Ztt.

picipes. Ztt.

celer. Mg. 
longicoruis. Mg. pubicornis. Ztt. confinis. Ztt. dissimilis. Fll. albiseta. $\mathrm{Pz}$. albicornis. TVlk. niveiseta. Ztt. mundus. TVlk. nigripes. $\mathbf{M g}$. nitidus. Mcq. brunnipes. Gimmerth. aeneus. Mcq. aeneicollis. Ztt. bivittatus. Meq. glaber. Mg. 1lavipemnis. IVIk. pulchellus. Wlk. formalis. Wlk. lineatus. $M \mathrm{~g}$. varipes. $\mathrm{Mg}$. taeniatus. $M g$. Meigenianus. Gimm. semihyalinus. Gimm. ambiguus. Mcq. paludosa. Perris. albipennis. Perris. 259. T'a chy drom i a. Mg.

\section{- Empis. H. -} Tachypeza. Mg. conmexa. Mg. cimicoides. Mg. ol. annulimana. Mg. arrogalns. L. cimicoides. F. arogans. Fll. Mg. umbrarum. Hal. albitarsis. Ztt. fuscipennis. Fll. sabulosa. Mg. calcanea. Mg. umbripennis. Mg. truncorum. Fll. nervosa. Mg:

tibialis. Meq.

nubila. Mg:

terricola. Ztt.

hyalipennis. Mcq.

Heeri. Ztt.

Winthemi. Ztt. fenestrata. Ztt. atriceps. Boh. praelusio. Wlk. morio. Wlk.

260. Elaphro peza. Meq. - Tachydromia. Ell. - Hemerodromi $\alpha . \mathbf{M g}$.

ephippiata. Fll.

261. C h e r s o d r o m i a. Wlk. - Empis. Ztt.

- Tachydromia. Fll.

- Tachypeza. Hal. arenaria. Hal.

brevipennis. Ztt. cursitans. Tht. hirta. Wlk. incana. IVIk. speculifera. Wik.

XXIII. Dolichopidae.

Rhaphinae.

262. A phrosylus. Wik. celtiber. Hal.

ferox. Wlk.

raptor. WIk.

venator. $\mathrm{Lw}$.

263. Machaerium. Hal. - Rhaphium. Lw. ol. - Smiliotus. I,w. maritimae. Hal. thinophilum. Lw.

264. R hap hium. Mg. Hydrochus. Fll. Porphyiops. Mg. pt. - Xiphandrium. Lw. fissum. 1,w.

caliginosum. Mg. macrocerum. $\mathrm{Mg}$. xiphias. Mg. nemorale. $\mathrm{Mg}$. pallitarse. Mcq. monotrichum. Lw. caliginosum. Ztt. macrocerum. Zitt. appendiculatum. $Z$ tt. cupreum. Wlk. auctum. Lw. quadrifilatum. Lw. lanceolatum. Lw. fasciatum. $\mathbf{M g}$. angusticorne. Lw. brevicorne. Curtis.

dissectum. Lw. sagax. Gerst. cupreum. Meq. albifrons. Ztt. tibiale. Perris. 265. Porphyrops. Mg. Rhaphium. Ztt. Lw. pt. - Hydrochus. Fll. - Anglearia. Carlier. Anglearia. Carl. antennatus. Carl. disciger. Stenh.

Schineri. Mik.

Porphyrops. Mg. spinicoxus. Lw. communis. IVIk. fascipes. Mg. latipes. Mcq. insulsus. W1k. nemorum. $\mathbf{M g}$. nigripes. Mcq. laticornis. Ztt. micans. Mg. nasutus. Fll. communis. Ztt. ol, 
penicillatus. IN. pectinatus. Lw. praerosus. Lw. suavis. Lw. elegantulus, Mg.

Wilsonii, Curtis. basalis. Lw. crassipes. $\mathrm{Mg}$. consobrinus. Ztt. fractus. Lw. obscuripes. Ztt. maculipes. Mg. longicormis. Fll. vitripennis. $\mathbf{M g}$. confinis. Ztt.

rufipes. Ztt.pt. scutellatus. Mg. fulripes. Mg: rujipes. $\mathbf{M g}$. ol. discolor. Ztt. communis. $\mathrm{Mg}$. gravipes. Wlk. thoracicus. Mg. rufipes. Mg: sulcipes. Mg. nitidus. Mcq. obscuratus. Mg. riparius. $\mathbf{M g}$. subnudipes. Ztt.

266. Syntormon. Lw. - Dolichopus. F.Porphyrops. Mg. Hydrophorus. Fll. Rhaphium. Mg. Ztt. - Plectropus. Hal.

- Syntormon und Synarthrus. Lw.

Synarthrus. Lw. pallipes. F. hammatum. ZJt. ensicorne. Mg. oedicnemus. I,W.
Šyntoxmon. Lw. denticulatum. Ztt.

liseriatum. Lw. spicatum. Lw. metathesis. Lw. Zelleri. Lw. pumilum. Mg. longiseta. Ztt. tarsatum. Fll. palmipes. $\mathbf{M g}$. gratiosum. Mg. punctatum. Ztt. monile. WVlk. vittatum. Meq. aculeatum. Ztt. pusillum. Ztt. pumilio. Ztt.

267. Systenus. Lw. - Rhaphium. Lw. ol. Scholtzii. Lw. adpropinquans. Lw.

Scholtzii. Lw. pt. bipartitus. Lw. leucurus. Lw. tener. Lw.

268. Achalcus. Lw. Rhaphium u. Porphyrops. Mg. - Dolichopus. Ztt.

cinereus. Wlk. pygmaeus. Ztt. flavicollis. Mg. pallidus. Ztt.

Dolichopinae. 269. Neurigona. Rond. - Musca. F. - Dolichopus. F. Fll. Medeterus. $\mathrm{Mg}$. Porphyrops. Mg. Plectropus. Hal. Saucropus u. Eutursus. Lw.
Eutarsus. Liv.

aulica. Mg.

Saucropus. Lw.

pallida. Fll.

ochracea. Mcq. suturalis. Flt. quadrifasciata. F. Erichsonii. Ztt.

quadrifasciata. Ell. abdominalis. Ell.

270. Xanthochlorus.

Lw. - Dolichopus.

W. Ztt. - Medeterus.

Mg. - Porphyrops.

Hal. WVlk.

ornatus. Hal.

tenellus. W.

flavellus. Ztt.

bicolorellus. Ztt. 271. Psilopus. Mg. Dolichopus. F. Leptopus. Fll. Sciapus. Zllr. platypterus. F. tipularius. Fll. crinipes. $\mathbf{M g}$. nervosus. Lehm. Wiedemanui. Fll. lobipes. Mg. lugens. Mg. longulus. Wlk. albifrons. $\mathbf{M g}$. contistans. Mg. contristans. IV. regalis. $\mathrm{Mg}$. flavicinctus. I, w. robustus. Lw. longulus. Fll. tenuinervis. Lw. euchromus. Lw. fasciatus. Mcq. 
obscurus. Mg.

laetus. $\mathrm{Mg}$.

eutarsus. Lw.

calceolatus. Lw.

spiniger. Ztt.

zonatulus. Ztt.

272. Hy p op hyllus. Lw.

-Dolichopus. Fll. -

- Hercostomus. Lw.

pt.

Hercostomus. Lw.pt.

longiventris. Lw.

Hypophyllus. Lw.

distans. Lw.

crinipes. Staeg.

pectinifer. Zllr.

discipes. Abrens. Lehm.

patellatus. $\mathrm{H} 11$.

patellipes. Mg.

ventralis. Ztt.

sphenopterus. Lw.

obscurellus. Fll.

plebejus. Mg.

appendiculatus. Mcq.

crinicauda. Ztt.

273. Sybistroma. Mg. - Dotichopus. Meq. nodicornis. Mg. setosa. Schin.

Dufourii. Mcq.

274. Haltericerus.

Rond.

impar. Rond.

eucerus. Lww.

spathulatus. Lw.

275. Dolichopus. Ltr.

- Musca. L. - Ne-

motelus. Deg. - Sa-

tyra. Mg. ol.

atripes. Mg. atratus. Mg.

maculipennis, Ztt. atratus. Ztt. ol.

fastuosus. Hal. picipes. Stann. plebejus. Ztt.

picipes. $\mathrm{Mg}^{\circ}$.

fulgidus. FII.

Meigenii. Lw. nigripes. Fll. pt.

Fallenii. Lw. nigripes, Fll. pt. melanopus. Stann. melanopus. $\mathrm{Mg}$. planitarsis. Fll. lepidus. Staeg. tibialis. Ztt. campestris. Mg. fulgidus. Ztt. nubilus. $\mathrm{Mg}$. inquinatus. Hal. latelimbatus. Mrq. vulgaris. Staml. excisus. Lw. albifrons. Lw. claviger. Stann. plumitarsis. Fll. confusus. Ztt. patellatus. Stam. discifer. Stamn. patellatus. Mg. confusus. Ztt. ol. plumipes. Scop. pennitarsis. Fll. simplex. Mg. Wahlbergi. Ztt. geniculatus. Stann. tibialis. Ztt. ol. armillatus. Whlbg. Stenhammari. Ztt. annulipes. Ztt. ol. discimanus. IVhlbg. discifer". $7 \mathrm{tt}$. sigilatus. Mg. pennatus. $11 \mathrm{~g}$. popularis. Fll. pt. ornatipes. $\mathrm{Lw}_{0}=\bar{\psi}$ popularis. IV. aemulus. Lw. acuticornis. IV. longicormis. Stamu. acuticornis. $\mathrm{Mg}$. nitidus. Fll. ornatus. Mg. jucundus. Hal. nitens. Stam. cilifemoratus. Meq. ritidus. Stam. griseipennis, Stanm. urbanus. Mg. argentifer. Lw. pictipennis. Whibg. siynifer. Lw. punctum. IVlk. sabinus. Hal. pictus. Staeg. simplex. Mg. thalassinus. Hal. virgultorum. WIk. arlustorum. Ztt. arbustorum. Stann. festivus. Hal.

Macquarti. Staeg. cilifemoratus. Ztt. trivialis. Hal.

intermedius. Staeg. linearis. Mg. parvulus. Ztt. agilis. Ztt. pallidicoxa. v. Ros. exiguus. Ztt. agilis. $\mathbf{M g}$. brevipennis. $\mathbf{M} \mathrm{g}$. migrans. Ztt. 
equestris. Hal. cinctus. Staeg. Staegeri. Ztt. aeneus. Deg. ungrulatus. F. Mg. chalybeus. Mg. analis. Meq. clavipes. Hal. vitriponnis. Staeg. trochanteratus. Ztt. fuscipes. Hal. tibiellus. Zitt.

Rothii. Ztt. ruficauda. Ztt. vitripemis. $\mathrm{Mg}$. brachycerus. Ztt. phaeopus. Wlk. remipes. Whlbg: argyrotarsis. Whlbg. fraterculus. Ztt. azureus. Meq. pectinitarsis. Stenh. Mamnerheimi. Ztt. modestus. Whlbg. micropygus. Whlbg. litorellus. Ztt. inconspicuus. Ztt. parvicaudatus. Ztt. ochripes. Ztt. caligatus. Whlbg. affinis. Whlbg. cinctipes. Whlbg. consimilis. Whlbg. cruralis. Whlbg. dissimilipes. Ztt. consobrinus. Ztt. atritibialis. Ztt. subrutilus. Ztt. groenlandicus. Staeg. grandicornis. Whibg. notabilis. Ztt. notatus. Staeg. nigritibialis. Ztt. misellus, Boh. flavipes. Stann. sublamellatus. Heq. guttipennis. Ztt. pictipennis. Boh. lineatocornis. Ztt. longitarsis. Stann. puncticornis. Ztt. punctum. Mg. rupestris. Hal. festinans. Ztt. fuscimanus. Ztt.

Ruthei. Lw. hilaris. Lw. fallaciosus. Gerst. brachycerus. Ztt.

bicolor. $\mathrm{F}$. minutus. F. gratiosus. Ztt. obscurellus. Ztt. ol. obscurellus. Mg. alpinus. Mg. cyaneus. $\mathrm{Mg}$. relictus. Mg: caerulcicollis. Mg. longicollis. $\mathrm{Mg}$. vividus. $\mathrm{MI} \mathrm{g}$.

276. Hy groceleuthus. Lw. - Dolichopus. Auct.

diadema. Hal. fraternus. Staeg. Stannii. Ztt.

bifurcatus. Heq. latipennis. Fll. Zetterstedtii. Stenh.

277. Tachytrechus. Stami. - Ammobates. Stanı. ol. - Dolichopus. Auct. ammobates. Wlk. plumipes. Fll. notatus. Stamn. plumipes. Mcq. litoreus. Hal. insignis. Stann. genualis. Lw. consobrinus. Curtis. plumipes. Mg. ripicola. Lw. melaleucus. Gerst.

278. Gymnopternus. Lw. - Dolichopus. Auct. - Musca. L. F. - Hercostomus. Lw. pt. nobilitatus. L. plumicornis. $1 \mathrm{Ig}$. apicalis. Ztt. ducalis. Lw. principalis. Lw. regalis. Mg: infucatus. Stanu. nigricornis. Mg. gracilis. Stann. bicolor. Mcq. Bohemanni. Whlbg. chaerophylli. $\mathbf{M g}$. germanus. Ztt. germanus. IV. conformis. $\mathrm{Lw}$. fumipennis. Stam. fuscipennis. Mg. laevifrons. Lw. pulchriceps. Lw. chrysozygos. IV. plagiatus. Lw. convergens. Lw. nigriplantis. Stann. Sahlbergi. Ztt. rusticus. Mg. obscuripes, $\mathbf{M g}$. nigripennis. Fll. 
exarticulatus. Lw. celer. Mg. sarus. Hal. cupreus. Fll. chalybeus. IV. metallicus. Stamn. nerosus, Fll. assimilis. Staeg: aerosus. Ztt. ol. parvilamellatus. Mcq. caudatus. Lw. inornatus. Lw. atrovirens. Lw. vivax. Lw. fugax. Lw. pilicornis. Stamn. nigrolamellatus. Mcq. nigrimaculatus. Curt. nanus. Mcq.

minimus. 'Ztt.

brevicomis. Staeg. obscuripennis. Ztt. angustifrons. Staeg. pratextatus. Hal. albifions. Ztt. piliter. Lw. appendiculatus. Lw. disopes. Gerst. grallator. Gerst. elegans. $\mathbf{M g}$. falvicaudis. IVlk. cretifer. Hal.

chetifor. WIk. alutifer. $11 \mathrm{lk}$.

279. Orthochile. Ltr. nigrocaerulea. Ltr. caerulea. Mcq. nigrocaerulescens. Stacg. soccata. Lw. unicolor. Lw. postica. Brullé.
Hydrophorinae. 280. Campsicnemus. Wlk. - Dolichopus. Fll.Ztt. -- Medeterus. Mg. - Camptosceles. Hal.

magius. Lw. compeditus. Lw. pusillus. Lw. platypus. Lw. scambus. Fll. prodromus. Mg. clavitibius. v. Ros. curvipes. Fll. cilitibius. v. Ros. loripes. Hal. femoralis. Ztt. armatus, Ztt. atomus. Ztt. paradoxus. Whlbg. umbripentis. Lw. alpinus. Wlk. punctipennis. Ztt. picticornis. Ztt. pilosellus. Ztt. pumilio, Ztt. lumbatus. Lw. marginatus. Lw. dasycuemus. Lw. filipes. Lw. varipes. Lw. articulatellus. Ztt. albilabris. Ztt.

281. H y d r o p horus. Whlbg. - Musca. L. - Dolichopus. F. Medeterus. Mg. Aphrozeta. Perris. Hydrophoins u. Scellus. Lw.
Hydrophorus. Lw. nebulosus, Fll. conspersus. Hal. inaequalipes. Meq. litoreus. Fll. pt. cinereus. Perris.

biseta. Lw. inaequalipes. WIk. praecox. Lehm. oceanus. Meq. litoreus. Fll. balticus. $\mathbf{M g}$. litoreus, Fll. pt. borealis. Lw. binotatus. Ztt. brunnicosus. Lw. rufibarbis. Gerst. alpinus. Whlbg. bipunctatus. Lehm. binotatus. kll.

Scellus. Iw.

notatus. F. armiger. Fll. spinimanus, $Z$ tt. notatus. Ztt. ol. dolichocerus. Gerst. 28\%. Liancalus. Lw.-Musca. Scop. - Me,deterus. Mg. - Hydrophorus. Ztt. Dotichopus. F. -Anoplomerus. Rond. virens. Scop. regius. $\mathbf{F}$. formosus. Hal. viridipes. Mcq. leucostomus, Lw. lacustris. Scop. virens. P'z. 
283. Th i $n$ o p hil us. Whlbg. - Rhaphium. Ztt. - Medeterus. Hal.

flavipalpis. Ztt. ruficornis. Hal.

maculico"nis. Ztt. versutus: WIk.

284. Peodes. Lw. forcipatus. Lw. 285.Teuchophorus.Lw.

- Porphyrops. Mg.

- Medeterus. Mg.

calcaratus. Meq. monacanthus. Lw. spinigerellus. Ztt. niyricosta. v. Ros. tlavicoxa. $\mathrm{Mg}$.

286. Sутрусииs. Lw. - Dolichopus. Fll. Porphyrops u. Medeterus. Mg.

cirrhipes. $1 \mathrm{~T}^{\top} \mathrm{lk}$. brevimanus. Lw. aeneicoxa. Mg. brevicornis. Ztt. pulicarius. Fll. anmulipes. Mg. pulicarizs. TVlk. cinerellus. Ztt. pygmaeus. Mcq. bifasciatus. Meq. fulviventris. Meq. bifasciellus. Ztt. spiculatus. Gerst. plantaris. Gerst. bicingulatus. Ztt.

287. Medeterus. Fisch. - Musca. I. - Dolichopus. F. - Hvdrophorus. Meq. Ztt. - Orthobates. Whibg. - Taechopates. Hal. obscurus. Ztt. signaticornis. Lw. ambiguus. Ztt. infumatus. Lw. melanopleurus. Lw. apicalis. Ztt. nigricans, Mg. tristis. Ztt. jaculus. Fll. tenuicauda. Lw. truncorum. $\mathbf{M g}$. diadema. L. rostratus. $\mathbf{F}$. aeneivittatus. Mcq. flavipes. Mg. plumbellus. Mg. minutus. Ztt. micaceus. Lw. muralis. Mg.

reneus. Mg. albiceps. $\mathbf{M g}$. bicolor. Mg. gratiosus. Mg. albipes. Ztt. nitidus. Mcq. pallipes. Ztt. jaculus. Ztt. ol. Diaphorinae. 288. Chry s otus. Mg. Musca. F. - Dolichopus. F. Fll. II. cupreus. Mcq. obscuripes. Ztt. laesus, IV. neglectus. W. viridulus. Fll. femoralis. Mg. taeniomerus. $\mathrm{Mg}$. gramineus. Fll. copiosus. $\mathrm{Mg}$. laesus. FH. pt. minimus. $\mathrm{Mg}$. suaris. Lw. cilipes. $\mathbf{M g}$. rufipes. Mg. melampodius. Lw. bicolor. Meq. femoratus. Ztt. signatus. Ztt. amplicornis. Ztt. nigripes. F. magnicornis. Ztt. raphioides. Ztt.

289. Chr ys o timus. Lw. - Dolichopus. Fll. - Chrysotus. Mg. molliculus. Fll.

laetus. Mg. concinmus. Ztt. 290. Thry p ticus. Gerst. smaragdinus. Gerst.

291. Anepsius. Lw. Porphyrops. Mg. Medeterus. Mg. flaviventris. $\mathbf{M g}$. 292. Argyra. Meq. Musca. F. - Dolichopus. F. Fll. Porphyrops. Mg. Argyra u. Leucostola. Lw.

Leucostola. Lw. vestita. IV.

$A r g y r a . \mathrm{Lw}$.

Hoftmeisteri. Lw. diaphana. $\mathbf{F}$. versicolor. $\mathrm{Mg}$. pellucens. Fll. pt. hirtipes. Curtis. confinis. Staeg. setimana. Lw. 
argentina. Mg. diaphano. Fll. argentella. Ztt. argentata. Meq. argyria. Mg. leucocephala. Mg. diaphana. Mg. pt. pellucens. Ztt. ol. fulgens. Hal. auricollis. Mg. pellucens. Fll. pt. festiva. Mg. atriceps. Lw. fulvipes, Hcq. spinipes. Mg. aristata. Gerst. grata. Lw.

magnicornis. Ztt. elongata. Ztt. incompta. Gerst.

293. Diaphorus. Mg. Dolichopus. Fll. Diaphorus u. Nematoproctus. Lw. Chrysotus. Mg. pt.

Nematoproctus. Lw. annulatus. Meq. distendens. Mg. longifilus. Lw. vitripemnis. Lw.
Diaphorus. Lw. tripilus. Lw. oculatus. Fll.

flavocinctus. Mg. Hofjmannseggi. Mcq. tuberculatus. Mg. bimaculatus. Mcq. Hoffmannseggi. Mg. nigricans. Mg. obscurellus. Ztt. cyanocephalus. Mg. Winthemi. Mg. disjunctus. Lw. latifrons. Lw.

\section{Diptera cyclorhapha.}

\section{A. Proboscidea.}

\section{A. Hypocera.}

XXIV. Ph orid a e.

294. Conicera. Mg. Phora. Mg. pt.

atra. $\mathrm{Ng}$.

dauci. $\mathrm{Mg}$.

similis. Hal.

293. G y m n o p h or a. Mcq.

- Phora. Mg. -

Trineura. Ztt. Fll.

arcuata. $\mathrm{Mg}$.

debilis. Hal.

996. Trineura. $\mathrm{Mg}$. -

Musca. F. - Tephritis. F. - Phora. Mg.

Plitodendria. Rond. aterrima. F. atra. Fll. stictica. Mg. velutina. Mg.

297. Phora. Ltr. Trineura. Mg. ol. Ztt. - Noda. Schellenb. flava. Fll. lutea. $\mathrm{Mg}$. interrupta, Ztt. subquadrifasciata. Ztt. fasciata. Fll.

atricapilla. Curtis. bicolor. Mg. semiflava. Hartig. brachyneura. Egg: aptina. Schin. rufipes. F. vulgaris. Fll. pt. pallipes. Ltr. annulata. Mg. heracleellae. Bouché. pulicaria. Fll. pygmaea. Ztt. sordida. Ztt.

? luctuosa. Mg. luctuosa. Mg. zonata. Ztt. humeralis. Ztt. ruficornis. Mg. minor. Ztt. gymnophorina. Ztt. carbonaria. Ztt. perennis. Mg: abdominalis, Fll. 
fulviventris, Bols.

flavicoxa. Ztt.

palposa. Ztt.

fuscipes. Meq.

ciliata. Ztt.

Giraudii. Egg.

maculata. Mg.

urbana. Mg.

trochanterata. Ztt.

opaca. Mg.

nigricornis. Egg.

pumila. $\mathbf{M g}$.

thoracica. Fll.

dimidiata. Mg.

notata. Ztt.

crassicornis. Mg.

erythrocera. Mg.

sordidipennis. L. Duf.

concinna. Mg.

florea. F.

palpina. Ztt.

funebris. Mg.

flexuosa. Egg.

distincta. Egg.

incrassata. Mg:

Bermuthi, Egg.

mordellaria. Fll.

femolata. Mg.

agilis. Mg.

obscura. Ztt.

carinifrons. Ztt.

caliginosa. Mg.

fuliginosa. Mg.

gracilipes. Mlg.

lugubris. Ng.

nigra. $\mathrm{Mg}$.

pusilla. Mfg.

sulphuripes. $\mathbf{M g}$.

nitidula. MIg.

liyalinata. Mg.

albipennis, $\mathbf{M g}$.

rapida. $\mathbf{M g}$.

nudifrons. Mcq. flavicornis. Mcq.

rufipennis. Meq.

flavipalpis. Heq.

scapularis. Meq.

vicina. Meq.

atra. Meq.

quadrata. Gimmerth.

bovista. Gimmerth.

sphingicidis. Bouché.

helicivora. L. Duf.

anceps. Ztt.

dubia. Ztt.

ritripennis, Mg.

galeata, Hal.

\section{B. Orthocera.}

\section{a. Oligoneura.}

XXY. Muscidae.

1. Muscidae acalypterae.

Borborinae.

298. C en chrid o b i a. Schin. - Cainus.

Egg.

Eggeri. Schin.

299. I i imosina. Meq. -

Copromyza. Fll.

Borborus. Mg.

Limosina. et He teroptera. Mcq. - Nerea. et Mycetia. R-Desv.

- Pterennis. Rond. pt.

Heteroptera. Mcq. acutangula. Ztt.

pusilla. Mg. heteroneura. Hal.
Limosina. Meq.

sylratica. Mg.

claripennis. R-Desv.

scutellata. Mg.

fontinalis. Fll.

arcuata. Mcq.

limosa. Fll.

lugubrina. Ztt.

riparia. R.-Desr.

lutosa. Stenh.

crassimana. Hal.

pygmaea. Ztt.

lugubris. L. Duf.

stercoraria. R.-Desf.

oelandica. Stenh.

pumilio. $M g$.

humida. Hal.

pusio. Ztt.

litoralis. Stenh.

ferruginata. Stenh.

ochripes. Mg.

abbreviata. Fll.

bifrons. Stenh.

coxata. Stenh.

pullula. Ztt.

fenestralis. Fll.

glabra. Mg.

pygmaea. MIg.

impressa. Mg.

tristis. Mg.

fulvipes. Mg.

rufipes. Mg.

clunipes. $\mathbf{M g}$.

flavipes. Mg.

sacra. Mg.

Hariceps. Ztt.

curtiventris. Stenh.

rerticella. Stenh.

nitens. Stenh.

aeneiventris. Stenb. 
anceps. Stenh. brericeps. Stenh. septentrionalis. Stenh. piligera. Stenh. zosterae. Hal. setulosa. Zitt. opacula. Stenh. brachystoma. Stenh. diadema. Stenh. curvinervis, Stenh. scutellaris. Hal. rufilabris. Stenh. vitripennis. $Z$ tt. fungicola. Hal. minutissima. Ztt. obtusipennis. Stenh. parrula. Stenh. leucoptera. Hal. nigerrima. Hal. aterima. Hal. melania. Hal. tarsata. Ztt. Stenhammari. Ztt. geniculata. Meq. erratica. Hal. quisquilia. Hal. spinipennis. Hal. fuscipennis. Hal. ragans. Hal. lugubris. Hal.

Pterennis. Rond. nivalis. Hal. 300. S pha erocera. Ltr. - Musca. F. Thagio. Schrk. Calobata. F. - Copromyza. Fll. - Borboirus. Mg: - Ceroptera. Mcq. pt. - Lordatia. R-Desv. subsultans. $\overline{\mathrm{F}}$. curvipes. Ltr. merdartm. R-Dest. stercoraria. R-Desv. cadaverina. R-Desr. necrophaga. R-Desv. monilis. Hal.

denticulata. Mg. vaporariorum. Hal. coprina. R-Desv. pusilla.. Fll.

scabricula. Hal.

crenata. Ztt.

obtusa. Mg:

opaca. Mg.

hyalipennis. $\mathrm{Mg}$. coronata. Ztt.

subcinerea. Brullé.

rufitarsis. Mg.

301. Borborus. Mg. Copromyza. Fll. Sphacrocera, Nerea u. Mycetia. R-Desv. -- Crunomyia, Apterina u. Olina. Mcq.

Apterina. Mcq. pedestris. $\mathrm{Mg}$.

Borborus. Mg. geniculatus. Meq. ater. Hal.

suillorum. Hal.

tibialis. R-Desv.

communis.R-Desr.pt. fuscipennis. Ztt. nitidus. $\mathrm{Mg}$.

hamaius. Hal.

niger. MIg. equinus. Fll.

communis, R-Dest. fuliyinosus. R-Desv: coprivorus. R-Desv. vulyaris, R-Desv. timetarius. $\mathrm{Mg}$. vitripennis. $\mathrm{Mg}$. saniosus. Westring. fumipennis. Stenh. costalis. Ztt. pallifrons. Fll. flavipennis. Hal. ater. Mg. stercorarius. Mg: varipes. $\mathrm{Mg}$. nervosus. $\mathrm{Mg}$. borealis. Ztt. tibialis. Ztt.

nigrifemoritus. IIcq. pallipes. Stenh. non $\mathrm{Mg}$.

sordidus. Ztt. glabrifrons. Mg: modestus. $\mathbf{M g}$. luridus. $\mathrm{Mg}$. pallidiventris, Mg. pallipes. Mg. rufipes. $\mathrm{Mg}$. lugens. Mg. incanus. Mg. punctipennis. Mca. longipes. Mcq. costatus. Mg. longipennis. Hal. nigrinus. Gimmerth. pilosiventris. Ztt. glacialis. Mg. 302. Therina. $\mathbf{M g}$. femoralis. Mg. nigra. Gimmerth. 303. Coelopa. MIg. Copromyza. Fll. Musca. F. frigida. Fll. F. simplex. Hal. eximia. Stenh. frigida. Hal. 
pilipes. Hal.

frigida. Mg.

parrula. Hal.

nitidula. Ztt.

nitidula. Lw.

gravis. Hal.

Phycodrominae.

304. Phycodrom a. Stenl. - Coelopa. Ztt. - Malacomyia. Hal.

fucorum. Ztt.

sciomyzina. Hal.

305. Rhicnoëssa. Lw.

cinerea. Lw.

306. Oedeparea. Lw.

buccata. Fll.

307. Orygma. $\mathbf{M g}$. - -

Psalidomyia. Doum.

luctuosa. Mg.

fucicola. Doumerc.

Thyreophorinae.

308. Thyreophora. Mg.

- Musca. Pz. F.

Seatophaga. Mg. pt. cynophila. Pz.

anthropophaga. R-

Desv.

furcata. F.

Scatophaginae.

309. S c a t op haga. Mg.

Musca. L. - Pyropa.

Illig. - Scatomyza.

Fll. - Scatina und

Amina: Rob-Desr.

Scatophaga. R-Desr.

analis. $\mathbf{~} \mathrm{g}$.

scybalaria.L. spurca. Mg.

suilla. Fll. pt.

lutaria. $\mathrm{F}$.

suilla. Fll. pt.

inquinata. $\mathrm{Mg}$.

stercoraria. I.

merdaria. F.

cinerea. Mg.

maculipes. Ztt.

suilla. F.

glabrata. Ztt. ol.

borealis. Ztt.

oceana. Mcq.

ostiorum. Hal.

serotina. Perris.

eximia. Hal.

Scatina. R-Dest.

litorea. Fll.

rufipes. Mg.

squalida. Mg.

fuscinervis. Ztt. ol.

incisa. Mcq.

lateralis, $\mathrm{Mg}$.

parisiensis. R-Desv.

claripennis. R-Dest.

nigricans. Mcq.

bipunctata. Meq.

fuscinervis. Ztt.

arrogans. Hal.

villipes. Ztt.

litorea. Ztt. ol. heteromyzina. Ztt. frontata. Ztt.

argus. Ztt.

antennata. Ztt.

validicornis, Ztt.

griseola. Fll.

tessellata. Meq.

turpis. Hal. rudis. $\mathrm{Hal}$.

calida. Hal.

decipiens. Hal.

ochrocephala. Brullé.

310. Fucellia. R-Desv.

- Scatophaga. MIg.

- Scatomyza. Fll. -

- Halithea. Hal.

fucorum. Fll.

arenaria. R.-Desp.

marina. Meq.

maritima. Hal.

affinis. Ztt.

muscaria. Ztt.

muscaeformis. Ztt.

muscina, Ztt.

signata. Ztt.

\section{Helomyzinae.}

311. Crymobia. Lw. Leria. Schin.

hiemalis. Lw.

longipennis. Schin.

312. Leria. R.-Desv. Helomyza. $\mathrm{Mg}$. Fll. - Blephariptera. Meq. - Orbellia. R.Desv. pt. - Scoliocentra und Blepha roptera. Lw.

Scoliocentra. Lw. villosa. $\mathrm{Mg}$.

Blepharoptera. Lw.

spectabilis. Lw.

caesia. Mg.

modesta. Mg.

serrata. L.

latrinarum. Deg.

fenestrarum.R.-Desr.

nigricornis. Mg. 
geniculata. Z Ztt. scutellaris. Ztt. serrata. F. ruficeps. $Z$ tt. serrata. Meq. biseta. Lw. iners. Mg. flavicornis. Lw. ruficornis, $\mathbf{M g}$. inscripta. Mg. variabilis. Lw. cinerea. Lw. crassipes. Lw. pusilla. Lw. alpina. Lw. cuniculorum. R.-Desw. domestica. R-Desv. fungivora. R,-Desv. nigricans. Mcq. rufa. Meq. ferruginea. Mg. melina. R.-Dest. mustellina. R.-Desf. subterranea. 1R.-Desv. halterata. Mg. canescens. Mg. sylvatica. $\mathrm{Mg}$. fuscana. Mg. Kaltenbachii. Mg. obscuriventris. Ztt. flavifrons. Ztt. Jumeralis. Zth. ruficauda. Ztt. myopiformis.R.-Desv. tibialis. 7tt. minuta. Ztt. fuscinervis. Ztt. simplex. Mg:

313. Heteromyza. Fll. - Heteromyza und Tephrochlamys. Lw. - Lentiphora. R.Desv. pt.
Tephrochlamys. Lw. magnicornis. Lw. tarsalis. Ztt. flavipes. Ztt. laeta. $\mathrm{Mg}$. rufiventris. Mg:

Heteromyza. Lw. atricornis. $\mathrm{Mg}$. cinerella. Mcq.

tigrina. Fll. scutellata. Meq. flareola, R-Desv. hilarella. Ztt. nigricornis. Mcq. opomyzina. Ztt. pygmaea. Ztt.

314. Thelida. R. - Desv. - Heteromyza. Fll. oculata. Fll.

filiformis. R-Desr. vespertilionea. R.-Desv. 313. Oecothea. Hal. Helomyza. $\mathrm{Mg}$. Eccoptomera u. Oecothea. I, w.

Eccoptomera. Lw.

ornata. Lw. longiseta, Mg. tlavotestacea. Ztt. flata. Lw. microps. Mg. myopina. Ztt. pallescens. Mg. excisa. Lw. emarginata. Lw.

Oecothea. Lw. fenestralis. Fll. fuscipennis. Mg. praecox. Lw.

microphthalma, Ztt. macrostyla. Meq. 316. G y momus. Lw. troglodytes. Lw.

317. Helomyza. Fll. Suillia. R.-Desr. Allophyla u. Helomy$z a$. Lw.

Allophyla. Lw.

atricornis. Mg".

Helomyza. Lw.

ls umilis. Mg: inornata. Lw. nemorum, $\mathbf{M g}$. variegata. Lw. rufa. Mg*

apicalis. Schin. pectoralis. Lw. maxima. Schin. gigantea. Lw. tuberivora. R.-Desr. hispanica. Lw. gigantea. Mg. tuberivora. Mcq. praeusta. Mg. dlava. Mg. rufa. Fll. pt. affinis. Mg. bistrigata. Mg. similis. Mg. tiguina. $\mathbf{M g}$. communis. R.-Desr. flava. Ztt. ol. rufa. Fll. pt. laevifrons. Lw. tigina. Ztt. univittata. . Ros. ustulata. $\mathrm{Ng}$. 
pilimana. Lw. pallida. Fll. Mg.

Zetterstedti. Lw.

montana. Lw.

olens. Mg.

pallida. Fll. pt.

testacer. Ztt.

vagiuata. $L w$.

parva. Lw.

femoralis. Lw.

rufa. Fll. Mg.

fungorum. R.-Dess.

griseola. $\mathrm{Mg}$.

hilaris. Zt.t.

notata. Mg.

lurida. $\mathbf{M g}$.

umbratica. Mg.

suilloidea, R,-Desv.

liliorum. R.-Desv.

lineata. R.-Desr.

nigriventris. Mcq.

maculata. Meq.

trifasciata. Curtis.

penicillata. L. Duf.

obscura. $\mathrm{Mg}$.

318. Curto not um. Meq.

- Helomyza. Perr.

- Diplocentra. Lw.

Perisii. Schin.

gibbum. Pelris.

319. Prosopomyia. Lw. pallida. Lw.

\section{Dryomyzinae.}

320. Lucina. Mg. -

Salticella. R.-Desv. fasciata. Mg.

saltitatrix. R.-Desv.

hispanica. Mg.

321. Actora. Mg

Helcomyza. Curt. aestuum. $\mathrm{Mg}$.

ustulata. Curtis.

mediterranea. Lw.

322. Dryomyza. Fll. -

Musca.F. - Dryope.

R.-Desr.

anilis. Fll.

liturata. R.-Desv.

flaveola. F.

vetula. Fll.

senilis. Ztt.

Zawadskii. Schum.

decrepita. Ztt.

fuscicornis. $\mathbf{M g}$.

praeusta. Mg.?

Sciomyzinae.

323. C or m o p t e r a Schin. - Sciomyza. Mg. - Graphomyzina. Mcq.

limbata. $\mathbf{M g}$. elegans. Mcq.

324. Sciomyza. Fll. Opomyza. Mg.pt. Colobaea. Ztt. pt. Dictya, Chaetocera, Arina u. Pherbellia. R.-Dest.

brevipennis. Ztt. glabricula. Ell.

angustipennis. Staeg. nigrimana. $\mathrm{Ig}$. glabricula. Fll. pt. albitarsis. Ztt.

ventralis. $\mathrm{Mg}$. atrimana. Ztt. testacea. Mcq. lata. Schin. simplex. Fll. clavipennis. R.-Dest. (Dictya). bifasciella. Fll. concentrica. Mg. pallida. Fll. dorsata. Ztt. albocostata. Fll. claripennis. R.-DesF. (Chaetocera).

obtusa. Fll. griseola. Fll. notata. Mg. austera. Mg. flaviceps. $M \mathrm{~g}$. analis. $\mathrm{Mg}$. anilis. Ztt. plumbella. $\mathbf{M g}$. fuscipes. Mcq. obscura. R.-Desv. dryomyzina. Ztt. fuscinervis. Ztt. ruficeps. Ztt. affinis. Ztt. atriseta. Lw. rufiventris. $\mathbf{M g}$. annulipes. Ztt. cinerella. Fll. herbarum. R.-Dest. dubia. Fll. nana. Fll. Schönherri, Fll. monilis. $\mathrm{Mg}$. vernalis. R.-Desv. ventralis. Fll. albiceps. Mg. acuticomis. 1 g. brumipes. $\mathrm{Mg}$. picta. $M g$. defecta. Mg. obsoleta. Mg. grisescens, $\mathbf{M g}$. pallidirentris. Fll. striata. Mg. 
bicolor. Ztt.

fumipennis. Ztt.

nasuta. Ztt.

pusilla. Ztt.

coxata. Ztt. ol.

rufa. Ztt.

thoracica. Ztt.

325. P la a e o m y i a. Schin.

- Musca.F. - Scalo-

phaga. F. - Scio-

myza. Fll. $\mathrm{Mg}$. -

Oscinis. Ltr.

nigripennis. F.

melanoptera. Ltr.

fuscipennis. $\mathrm{Mg}$.

leptiformis. Schin.

Tetanocerinae.

326. Ectinocera. Ztt.

borealis. Ztt.

327. Tetanocera. Fll.

- Nusca. L. F. Scop.

- Scatophaya. F. -

Dictya. E.

elata. $\mathrm{k}$.

laevifrons. Lw.

sylvatica. $\mathrm{Mg}$.

hyalipennis. $\mathrm{v}$, Ros.

unicolor. Lw.

robusta. Lw.

arrogans. Ztt.

ferruginea. Fll.

stictica. R.-Desv.

arrogans. Mg.

praeusta. v. Ros.

quinquemaculata. v. Ros.

vittigera. Schumm.

punctata. F.

reticulata. F.

obsoleta. Fll.

umbrarum. L. punctulata. Scop.

hieracii. F.

umbrarum. F.

argus. F.

gentilis. R.-Desp.

nemorum. Fll.

gemmata. Ahrens.

formosa. Lw.

coryleti. Scop.

chaerophylli. F.

variegata. Fll.

media. Hal.

vittata. Hal.

albipennis. Gimmerth. 328. Limnia. R.-Desv.

- Musca. F. Scop.

- Scatophaga. F. -

Tetanocera. Fll. Mg.

marginata. F.

crinicornis. Fll.

stictica. F.

cincta. F.

catenata. Lw.

Zelleri. Lw.

irrorata. Meq.

fenestrata. Mcq.

Mannii. Schin.

unguicornis. Scop.

flavifrons. $\mathrm{Pz}$.

pratorum. Fll.

limbata. R.-Desv.

rufifrons. $\mathrm{F}$.

fumigata. Scop.

recta. Lw.

recticulata. Fll. pt.

rufifrons. Ztt.

obliterata. F.

nubila. Lw.

bivittata. Meq.

trivittata. Lw.

albitarsis. Gimmerth.
329. Elgiva. Mg. Insca. F. Scop. Dictya. F. - Tetanocera. Fll. - Chione u. Hydromyia.R.Desr. - Ilione. Hal. albiseta. Scop. aratoria. $\mathrm{F}$. interstincta. Fll. communis. R.-Desv. dorsalis. $\mathrm{F}$.

punctipennis. Fll. sepenedoidea. R.-Dsv. caeruleipennis. R.-

Desr.

lineata. Fll.

cucularia. F. pt.

rufa. Pz.

cucularia. F. pt.

divisa. Lw.

cucularia. L.

punctithorax. v, Ros. trifaria. Lw.

sciomyzina. Ztt.

330. Sepedon. Ltr. -

Syrphus, Nulio und

Bacha. F. - Scato-

phaga. F. - Musca.

$\mathrm{Pz}$. Scop.

sphegeus. F.

rufipes. $\mathrm{F}$.

palustris. Ltr.

flavipes. Schrk.

spinipes. Scop.

Haefneri. Fll.

ferrugineus. L.-Duf.

hispanicus. Lw.

Geomyzinae.

331. Leptomyza. Mcq.

- Anthomyza. Fll. Anthophilina. Ztt. "pomyza. Ng. pt. 
pallida. Z Ztt.

gracilis. Fll.

socculata. Ztt.

sordidella. Ztt.

frontalis. Fll.

grisea. Fll.

laeta. Mg.

flavipes. Ztt. gracilis. Fll. pt.

puberula. Ztt.

nigrina. Ztt.

bicolor. Ztt.

flavella. Ztt.

cinerella. Hal.

332. Geomyza. Fll. Tephitis. F. - Opomyza. Mg. pt. Musca. L.

bimaculata. $\mathbf{M g}$. combinata. L. consobrina. Ztt, ol. maculata. Germ. venusta. $\mathrm{Mg}$. tripunctata. Fll. marginella. Fll. nitida. Mg. circumdata. Mg. apicalis. Mg. sabulosa. Hal. brevipennis. Ztt. cingulata. Hal. angustipennis. Ztt. unguicella. Ztt. unipunctum. Ztt. griseola. Ztt. terminalis. Ztt.

333. Opomyza. Fll. Musca. L. - Dacus u. Tephritis. F. germinationis. L. Nataliae. Schin. florum. E. punctella. Fll. nigriceps, $\mathrm{Mg}$. fasciata. Mcq. bilineata. Mcq. fuscipennis. Meq. guttipennis. Ztt. tremula. Hal.

distincta. $\mathrm{Mg}$. sororcula. Mg. atrimana. $\mathrm{Mg}$. albimana. Mg. rufipes. $\mathrm{Mg}$. glabra. Mg. leucopeza. Mg. bicolor. $\mathrm{Mg}$. modesta. Mg. nigrimana. $\mathrm{Mg}$. flaripes. $\mathbf{M g}$. scutellata. Rob. asteia. Hal. pectoralis. Ztt.

334. Scyphella. R.Desv. - Musca. L. - Sapromyza. Fll. Mg. - Chyromyia und Scyphella. R.Desv. - Thyrimyza. Ztt.

lutea. Fll.

flava. $\mathrm{F}$.

niuricornis. R-Desr interstincta. Ell. flava. L. consobrina. Ztt. fenestrarum. R.-Desr. femorella. Fll. bipunctella. Ztt. chrysophthalma. Ztt. 335. Diastata. Mg. Geomyza. Fll. obscurella. Fill. nebulosa. Fll. ornata. Mg. fumipennis. Mg. punctum. $\mathbf{M g}$. costata. Mg. juscula. Fll. marginella. Ztt. ol. basalis. Mg. anus. Mg. adusta. Mg. obscuripennis. $\mathbf{1 f g}$. luctuosa. Mg. marginalis. $\mathrm{Mg}$. rufipes. $\mathrm{Mg}$. rufitarsis, Mg. diadema. Mg. apicalis. Rob. capitata. Rob. frontalis. $\mathrm{Mg}$. striata. Rob. nitida. Mg. fulvifrons. Hal. claripennis. Meq. unipunctata. Ztt.

Leptopezina. Meq. gracilipes. $\mathrm{Mg}$.

Drosophilinae. 336. Asteia. Mg. amoena. Mg. concinna. $\mathrm{Mg}$. elegantula. Ztt. 337. Periscelis. Lw. Notiphila. Fll. Ephydra. Mg. Diosophila. Zitt. amulipes. Lw. amulata. Fll. Wimnertzii. Egg. nigra. Ztt.

338. Stegana. Mig. -Musca. Scop. - Drosophila. Fll. 
coleoptrata. Scop.

lypoleuca. Mg.

annulata. Hal.

curvipeunis. Fll.

furta. WVlk.

339. Phortica. Schin.

- Drosophila. Hll.

variegata. Fll.

340. Gitolla. Mg.

distigma. Mg:.

341. Dros ophila. Fll. -

Musca. L. - Camilla.

Hal. - Scatomyza.

IIardy. $p$ t.

Cumilla. Hal.

golabr. Fh.

Drosophila. Fll.

maculata. 1.. J)uf.

transversa. Ell.

phalerata. Mg.

distincta. Egg.

obscura. Fll.

melanogaster. Mg.

niuriventris. Ztt.

approximata. Ztt.

fasciata. Mg:

histrio. $\mathbf{M g}$.

fenestrarum. Fll.

niticliventris. Mcq.

virginea. Mg.

funchris. $\mathrm{k}$.

erythrophthalma. Pz.

cellaris. Mcq.

confusa, Staeg.

fenestrarum. $\mathrm{Mg}$.

funchis. Fll. pt.

tristis. Fll.

cinerella. Fll.

rufipes. $\mathrm{Mg}$.

litoralis. $\mathrm{Mg}$. nigrimana. Mg.

fuscimana. Ztt.

nigriceps. Mg.

varipes. Meq.

alboguttata. Whlbg.

albilabris. Ztt.

spurca. Ztt.

costata. Zitt.

congesta. Ztt.

picta. Ztt.

caminaria. Hal.

pallipes. L. Duf:

nuve-punctata. L. Duf. aceti. Heeger.

Scaptomyza. Hardy.

flaveola. M $\mathrm{g}$.

flava. Curtis.

apicalis. Hardy.

pallida. Ztt.

graminum. Fll.

sordida. Ztt.

tlaripennis. Ztt.

griseola. Ztt.

incana. Mg.

Hava. Hll.

maculipennis. Gimn .

34\%.A u la cigaster. Meq.

- Notiphila. Fll. -

Apotomella. L. Duf. -

Ampycophora. Whlbg.

- Diastala. Mg.

rufitarsis. Mcq.

tarsata. IVhlbg.

impiessifrons. L. Duf.

leucopeza. Mg.

Ephydrinae.

Notiphilinac.

343. Dichaeta. Mg. -

Notiphila. Ell.

caudata. Fll.

brevicauda. Lw.

tibialis. Brullé.
344. Notiphila. Fll. Keratocera. R.-Desr. uliginosa, Hal.

tarsata. Stenh. nigricornis. Stenh. nigra. R.-Desv. stagnicola. R.-Desv. guttiventris. Stenh. maculata. Stenh. renusta. Lw. riparia. $\mathrm{Mg}$. australis. Lw. cinerea. Hll.

palustris. R,-Desv. major. Stenh. dorsata. Stenh. ammulipes. Stenh.

34. Trimerina. Meq. - Noliphila. Mg. -

Hydrellia. $\mathrm{Mg}$. Psilopa. Fll.

madizans. Fll.

niyella. Lw.

tibialis. Meq.

346. Discomyza. Mg.

- Notiphila. Mg.-

Psilopa. Hll.

incurva. Fll.

cimiciformis. Hal.

marginelia. Fll.

34\%. E p hy gr o b i a. Schin.

- Notiphila. Mg. -

Psilopa. Hll. Lw. -

Hydrellia. Mcq. Perr.

- Hygrella. Hal.

leucostoma. Mg.

nana. Lw.

apicalis. Perris.

compta. Mg.

nitidula. Fll.

obseuripes. Lw.

polita. Meq.

tarsella. 7tt. 
nigritella. Stenl.

plumosa. Fll.

longula. Stenh.

3i8. Clasiopa. Stenh.

- Notiphila. FII. Mg.

- Discocerina. Meq.

Lw.

obscurella. Fill.

nigrina. Mg.

cinerella. Stenl.

calceata. Mg.

nigrina. Stenl.

pulicaria. Hal.

fuscella. Stenh.

glabricula. Fil.

pallidula. Stenh.

3.9. A thy rog los sa.Lw.

- Notiphila. Mg.

glabrạ. Mg.

3:50. Hecamede. Hal. -

Notiphila. Mg.

Clasiopa. Stenh.

albicans. $\mathrm{Mg}$.

globifera. Boh.

lateralis. Lww.

glaucella. Stenh.

costata. Lw.

\section{Hydrellinae.}

33 1. Glenanthe. Hal. ripicola. Hal.

332. H y d r ellia.R.-Desr.

- Notiphilla. Fll, u.

Auct.

geniculata. Stenh.

caesia. Stenh.

albilabris. Mg.

argyrostoma. Stenh.

argyria. R.-Desv.

thoracica. Hal.

frontalis. Tw. mutata. Ztt. plumosa. Stenh.

concolor. Stenh. cinerascens. Hcq.

pilitarsis. Stenh.

laticeps. Stenh. cardamines. Hal. pt.

flavilabris. Stenh.

cardamines. Hal. pt.

fulviceps. Steuh.

grisea. Stenh.

discolor. Stenh.

flaviceps. WVlk.

flaviceps. Mg.

aurifacies. R.-Desv.

hydrocotyles. Hal.

porphyrops. Hal.

griseola. Fll.

communis. R.-Desv. chrysostoma. $\mathrm{Mg}$.

viridescens. R.-Dest.

nigripes. R.-Desv.

nigripes. Ztt.

flavicomis. Stenh.

erythrostoma. WIk.

flavicornis. Fll.

eruthrostoma. $\mathbf{M g}$.

cochleariae. Hal.

modesta. Lw.

ranunculi, Hal.

incana. Stenh.

nympheae. Stenh.

fusca. Stenh.

nigricans. Stenh.

maura. Mg.

nigrina. Lw.

albifrons. Fll.

obscura. Mg.

albiceps. $\mathbf{M g}$.

fuliginosa. R-Desr.

pusilla. Mg.

taripes. Mcq.

fulvipes. Mcr. lineata. $\mathbf{M} \mathrm{cq}$.

lucida. Meq.

viridis. Mcq.

lapponica.!Stenh.

alboguttata: Lw.

aunulata. Lw.

posticata. Mg.

nigriceps. Mg.

brunnifacies.R.-Desv.

pallipes. $\mathbf{M g}$.

pulchella $\mathrm{Mg}$.

flaviventris. Mg:

rufipes. $\mathbf{M g}$.

tristis. Mg.

nigripennis. $\mathbf{M g}$.

affinis. $\mathbf{M g}$.

litorella. $\mathbf{M g}$.

amoena. Mg.

rufitarsis. Mg.

ruficornis. Mg.

bicolor. Mig.

opaca. Mg.

ruficeps. Mg.

maritima. Perris.

lepida. Mg.

353. Atissa. Hal. -

Ephydra. Hal. ol.

pygmaea. Hal.

ripicola. Lw.

3oั4. Philygria. Stenh.

- Notiphila. Fll. -

Ephydra. $\mathrm{Ig}$. - Hy.

drina. R.-Desr.

picta. Fll.

pullula. Fll.

punctatonervosa. Ell.

stictica. Mg.

interrupta. Hal.

femorata. Stenh.

flavipes. Fll. pt.

Haripes. Fll. 
vittipennis. Ztt.

posticata. Mg.

nigricauda. Stenh.

interstincta. Fll.

maculipennis. R.-

\section{Desr.}

abdominalis. Stenh.

trinervis. Stenh.

35̋. Hya dina. Hal. -

Notiphila. Fll. -

Ephydra. Mg. -

Philygria. Stenh. pt. scutellata. Hal.

guttata. Fll.

vernalis. R.-Dest.

nitida. Meq.

brevicornis. Ztt.

grettata. Stenh. pt.

356. Axysta. Hal.

Ephydra. Hal. ol. --

Notiphila. Ztt.

Trimerina. Meq. -

Hydrina. R.-Desy. cesta. Hal.

caeruleiveritas. Mes: punctulata. Stenh.

viridula. R,-Desv.

$$
\text { Epleytrinat. }
$$

357. Canace. Hal.

nasica. Hal.

358. Pelina. Hal. -

Notiphila. Fll. -

Ephydra. Mg. -

Telmatobia. Stenh.

aenea. Fll.

glabricula. Mg.

aenescens. Stenh.

guttipennis. Stenh.

359. Ochthera. Ltr. Musca. Deg. F. Tephritis. F. - Macrochira. Mg. mantis. Deg.

manicata. F.

mantispa. Lw.

Schembrii. Rond.

360. I'ary dra. Stenh. -

Ephydra. Fll. Auct.

- Napaea. R.-Desv.

pubera. Lw.

pusilla. Mg.

infecta. Hal.

nasuta. Stenh.

fossarum. Hal.

affinis. Stenh.

quadripunctata. $\mathbf{M g}$.

furcata. Steuh.

aquila. Fll.

coguata. Lw.

coarctata. Fll.

hecate. Hal.

litoralis. $\mathbf{M g}$.

stagnicola. R.-Desv.

rufitarsis. Meq.

361. Halmop ota. Hal.

Ephydra. Bouché.

salinarum. Bouché.

salinaria. Lw.

mediterranea. Lw.

362. Ep hydra. Fll.

bivittata. Lw.

riparia. Ell.

albula. $\mathrm{Mg}$.

salinaria. v. Heyd.

halophila. v. Heyd.

macellaria. Eg*g.

salinae. Ztt.

micans. Hal.

riparia. Mg.

aurata. Stenh.

breviventris. Lw.

curvicauda. Mg.

lacustris. $\mathbf{M g}$. puludum. Mig.

quadriguttata. Mg.

subguttata. Mg.

glauca. $\mathrm{Mg}$.

melanostoma. Mg.

pictipennis, Mg.

minuta. Mg:

rufipes. Mg.

basilaris. $\mathrm{Mg}$.

pallipes, Mg.

ochrostoma. Brullé.

orichalcea, Gimmerth.

maculipennis. Gimm.

aeneiventris. Mcq.

irrorata. Mcq.

fuscipennis. Meq.

psilopina. Ztt.

363. Ilythea. Hal. -

Ephydra. Ztt. -

Epipela. Stenh.

spilota. Curtis.

notata. Stenh.

364. Ca enia. R. - Desv.

- Ephyitra. Auct.

defecta. Hal.

albidipennis. Stenh.

obscura. Mg.

palustris. Fll.

caricicola. R.-Desv.

fumosa. Stenh.

riparia. Fll.

365. Sc a tella. R.-Desv.

- Ephydra. Auct.

quadrata. Fll.

graminum. Hal.

Stenhammari. Ztt.

sibilans. Hal.

nubilipennis. Stenh.

dichaeta. Lw.

sorbillans. Hal.

argyrostoma. Stenh.

aestuans. Hal.

tlavipennis. Stenh. 
silacen. Lw. stagnalis. Fll.

buccata, R.-Desv.

lutosa. Hal.

flavescens. Stenh. cribrata. Stenh. cariceps. Stenh. despecta. Hal.

fenestrata. Stenh. signata. Lw. variegata. Lw. laevigata. Lw. flavitarsis. Ztt. pumilio. Lw. contaminata. Stenh. vittigera. Ztt. planiceps. Boh. noctula. Mg. aestivationis. IVlk. leucostoma. $\mathrm{Mg}$. compta. Hal. megastoma. Ztt. 366. Teich omyza. Meq.

- Scatella. R.-Desv.

- Ephydra. Mg. Tichomyza. Lw. fusca.Mcq.

urinaria. R.-Desv. quinquepunctata. Gimm.

longipennis. $\mathrm{Mg}$.

Chloropinae.

367. Eurina. Mg. calva. Egg. pubescens, Mg. lurida. Mg. clypeata. $\mathrm{Mg}$. 368. Pla tyc e pha la. Fll. - Musca. F. - Oscinis. F. - Tetano$\operatorname{cer}^{2}$ a. Ltr. umbraculata. F. agrorun. Fll. planifrons. F. culmorum. Fll. nigra. Mg. 369. Homalura. Mg. tarsata. Mg. flava. Brullé. 370. Camarota. Mg. flavitarsis. $\mathrm{Mg}$. aurifrons. Hal.

371. Selachops. Whlbg. - Encoelocera. Lw. flarocincta. Whlbg. bicolor. Lw.

372. Meromyza. Mg. - Musca. L. F. Tephritis. E. - Oscinis. Fll. pratorum. Mg.

smaragdina. L. Duf. viridula. Hal. saltatrix. L. minuta. F. clavicrus. Schrk. variegata. Mg. saltatiix. Fll. pt. laeta. Mg. nigriventris. Mcq. femorata. Mcq. 373. Chlorops. Mg. Musca. F. - Oscinis. Fll. Ztt. cingulata. $\mathrm{Mg}$. Scholtzii. Egg. rufina. Ztt. gracilis. Mg. geminata. $\mathbf{M g}$. pulchra. Schin. didyma. Ztt. nasuta. Schrk.

lineata. FIl. pt. umbelliferarum.Schk. scalaris. Mg. laeta. Mg. fasciata. Mg. hypostigma. Mg. confluens. Mg. notata. Mg. glabra. Mg. circumdata. Mg. lineata. F.

pumilionis. $\mathrm{F}$. taeniopus. Mg. strigula. Mg. pt. strigula. F. brummipes. Ztt. lucida. Mg. pygmaea. Mg. simplex. Mg. geniculata. Mg. Cereris. Fll. scutellata. Pz. messoria, Fll. tarsata. Fll. albitarsis. Mg. gentilis. Mg. interrupta. Mg. calceata. Mg. speciosa. Mg. frontosa. Mg. cinctipes. $\mathrm{Mg}$. limbata. Mg. elongata. Mg. ornata. $\mathrm{Mg}$. lineola. Brullé. lateralis. Hal. scutellaris. Ztt. agnata. Hal. fulvifrons. Hal. erythrocephala. Ztt. ruficeps. Ztt. ol. brunnicormis. Meq. flavifrons. Meq. nigrimana. Mcq. rufiventris. Mcq. 
rufa. Mcq. taeniata. Meq. analis. Mcq.

nigrimana. Meq. (bis) albiseta. Mcq. lateralis. Mcq. annulipes. Mcq. trifasciata. Ztt:

lasta. Ztt. ol. lineola. Zit. longicornis. Ztt. obscurella. Ztt. parvula. $Z$ tt. currinelvis. Ztt. approximatonervis. Ztt. troglodytes. Ztt. citrinella. Zitt. figurata. $Z \mathrm{tt}$. puncticollis. Ztt. nigriventris. Meq. annulipes. Brullé.

37\%. Siphonella. Mcq. - Musca. Bjerkand.

- Madiza. Fll. pt.

- Chlorops. Mg. Oscinis. Lw. pt. pumilionis. Bjerk. flavella. Ztt. laevigata. Fll. oscinina. Ztt. palposa. Ztt. nucis. Perris. aprica. $\mathrm{Mg}$. oscinina. Fll. nitida. $\mathrm{Mg}$. tristis. Lw. trilineata $\mathrm{Mg}$. ruficornis. Meq. aenea. Meq. ruficeps. Mcq. ormatifrons. Lw. dasyprocta. Lw. obscurifrons. Lw. marginata. Lw. 375. Oscinis. Ltr. Musca. L. F. -

Chlorops. Mg: pt. albiseta. $\mathrm{Mg}$. maura. Fll. vindicata. Mg: nitidissima. $\mathbf{M g}$. ruficeps. MIg: flavitarsis. Mg. frit. $\mathrm{I}$.

hordei. Bjerk. pusilla. Mg. frit. Fll. $p^{\text {tt. }}$ longula. Mg. plumigera. Mg*. lineella. Fll. cincta. $\mathrm{Mg}$. frontella. Fll. pratensis. Mg. rutipes. Mg. vitripennis. $11 \mathrm{~g}$. cognata. Mg. albipalpis. Mg. sulcicollis. $\mathrm{Mg}$. atra, $M g$. fascipes. $\mathbf{M g}$. glaberrima. $M g$. vagans. Mg: hyalipennis. Mg: nigrita Mg. picta. $M g$. amoena. Mg. gilvipes. Lw. laevifrons. Lw. fumipennis. $\mathbf{M g}$. phaeoptera. $\mathrm{Mg}$. albiceps. Mg. fasciola. Mg: socia. $\mathrm{Mg}$. anthracina. Mg. lepida. Mfg. angustifrons. Mg. diadema. Mg. quadrivittata. Mg: varipes. Mg. annulata. Mg. annulipes. Meq. flava. Meq. flavofemorata. Meq. fuscipes. Meq. pallidirentris. Meq. bicolor. Mcq. bipunctata. Mcq. obliqua. Meq. fulviventris. $M g$. rufiventris. Meq. rufimana. Meq. brunnitarsis. Meq. tibialis. Mcq. flavimana. Meq. nigerrima: Heq. riridescens. Mcq. polita. Meq. rufitarsis. Mcq. dubia. Meq. atricornis. Ztt. atricilla. Ztt. nana. Ztt. basalis. Ztt. sulcella. Ztt. nigripes. Ztt. anmulifera. Ztt. cinctella. Ztt. sordidella. Ztt. fasciella. Ztt. brachyptera. Ztt. abdominalis. Ztt. ephippium. Ztt. maculipennis. Ztt: humeralis. Ztt. brevirostris. Lw. 
3\%6. Elachiptera. Meq.

- Oscinis. Fll. Chlorops. MIg. Crassiseta. v. Ros.

Lw. - Myrmemorpha. L. Duf.

brevipennis. $\mathbf{M g}$.

brachyptera. L. Duf. cornuta. Fll.

femoralis. $\mathrm{Mg}$.

fuscipes. V. Ros.

annulipes. v. Ros.

flaviventris. v. Ros.

megaspis. Lw.

377.G a m p ocera. Schin.

- Chlorops. Heeg.

numerata. Heeg.

378. Lipara. Mg.

Gymnopoda. Mcq.

lucens. $\mathbf{M g}$.

tomentosa. Mcq.

rufitarsis. Lw.

similis. Schin.

379. Mosillus. Ltr.

Syrphus u. Eristalis.

F. - Llidia. Meq.

Gymnopa. Mg.

aeneus. Fll.

arcuatus. Ltr.

niger. Mg.

aeneus. Mg.

subsulians. $\mathrm{F}$.

albipenuis. Lw.

$$
\text { Psilinae. }
$$

380. Psila. Mg. - Musca.

L. F. - Scatophaga.

Fll. Ztt. - Psilom yia.

Ltr. - Oblicia. R.-

Desv,

obscuritarsis. Lw. fimetaria. $\rfloor$.

flava $\mathrm{Pz}$.

testacea. R.-Desv.

rufa. Scop.

rufa. $M g$.

pallida. Fll.

abdominalis. Schum.

bicolor. Mg.

debilis. Egg.

pectoralis. $\mathrm{Mg}$.

humeralis. Ztt.

atrimana. $\mathrm{Mg}$.

fuscinervis. Ztt.

morio. Ztt.

gracilis. Mg.

rosae. F.

nigricornis. $\mathbf{M g}$.

villosula. $\mathrm{Mg}$.

atra. Mg.

nigra. Fll. pt.

nigra. Fll. Mg.

signata. Fll.

buccata. Fll.

latipalpis. Ztt.

ephippium. Ztt.

unilineata. Ztt.

limbatella. Ztt.

tarsella. Ztt.

intermedia. Mcq1.

381. Psilos oma. Ztt. -

Scatophaga. Ztt. ol. Audouini. Ztt.

Lefebvrei. Ztt.

dispar. Schumm.

382. Chyliza. Fll. Musca.Pz.-Sargus.

F. - Cordylura. Mg.

pt. - Dasyna. R.-

Desr.

atriseta. Mg.

annulipes. Meq.

fuscipennis. R.-Desv.

obscuripennis. LW. leptogaster. P'z.

scutellatus. F.

Peleterii. R.-Desr.

vittata. $\mathbf{M g}$.

analis. Mg.

varia. Mg.

pumila. Meq.

ustulata. Ztt.

gracilis. Lw.

383. Lox o era. Mg. -

Miesca. I. - Syrphus. Mulio. $\mathrm{r}$ : -

Nemotelus. Schrk.

elongata. $\mathbf{M g}$.

ichneumonea. Ztt.

fulviventris. Mg.

nigrifrons. Meq.

dorsalis. Lw.

ichneumonea. L.

aristata. $\mathrm{Pz}$.

albiseta. Schrk.

confusa. Ztt.

sylvatica. Mg.

381. Platystyla. Mg.

- Loxocera. Mg"ol.

Hoffmannseggii. Mig:

Tanypezinae.

385. Tetanura. Fll.

pallidiventris. Fll.

386. T'any peza. Fll.

longimana. Fll.

387. Micropeza. Mg. -

Musca. L. F' - Ca-

lobata. F. Itr. -

Phantasma. R.-Desv. corrigiolata. L.

filiformis. $\mathrm{F}$.

Iateralis. Mg.

thoracica. R.-Desr.

Kawallii. Gimmerth. 
388. Calobata. Mg. - 393. Piophila. Fll. Musca. L. Pr. - Musca. L. E - TeNerius. R.-Desv. pt. phritis. F. - Tyrocalceata. Fll. ephippium. F. inulue. R.-Desv. cibaria. L. cothurnata. Fll. solidaginis. R.-Desv. soror. R.-Dest. cothurnata. Pz.

nigricomis. Ztt. petronella. L. corrigiolata. Fll. trivialis. Lw. femoralis. MIg. palustris. Mg: sellata. $\mathrm{Mg}$.

\section{Sepsinae.}

Anomalae.

389. Lissa. Mg. - Ocy. ptera. F. - Chyliza. Ell.

loxocerina. Fll. dolium. F. rufipes. Gimmerth. 390. Rhy n chaea. Ztt. lonchaeoides. Ztt.

391. Madiza. Fll. Gymnopa. Mg. pt. glabra. Fll. rufitarsis. $\mathbf{M g}$. alunulitarsis. $\mathrm{Ztt}$. 392. Mycetaulus. Lw. - Opomyza. $\mathbf{M g}$. Geomyza.Fll. - Piophila. Ztt.

bipunctatus. Fll. Hoffmeisterix. Lw. phaga. Kirby. nigriceps. $\mathbf{M g}$. vulgaris. Fll. pt. foreolata. Mg. aftinis. Mg. pygmaea. Ztt. ol. vulgaris. Hll. pt. casei. I. atrata. F. vulgaris. Fll. pt. petasionis. L. Duf. pusilla. Mg. continis. Mg. varipes. $\mathbf{M g}$. vicina. Mg. latipes. $\mathrm{Mg}$. laevigata. Mg. flavitarsis. Mg. nigrimana. Mg. nigricornis. Mg*. distincta. Mg. ruficoxa. Mcq. viridis. Mcq. metallica. Brullé. nigra. Brullé. nitida. Brullé. pilosa. Staeg. lonchaeoides. Ztt. llavipes. Ztt. caerulescens. Ztt. punctipennis. Ztt. luteata. Hal. apii. Westw.

$$
\text { Sepsinae. }
$$

394. Saltella. R.-Desv. - Piophila. Fll. Nemopoda u. Anisophysa. Meq. - Pandova. Hal. scutellaris. Fll. ruficoxa. Meq. ferruginea. R.-Dest. scutellata. Meq. ? sellata. Hal. ? pectoralis. Ztt. nigripes. R.-Desv. basalis. Hal. albipennis. Mcq. 395. Themira. R.-Desv. - Musca. L. F. Tephritis. F. - Sepsis. Auct. - Cheligaster. Meq. - Themira u. Halidaya. Rond.

Cheligaster. Mcq.

putris. L.

fimeti. Schrk.

Leachii. Mg: coxarum. Ztt. ol. ciliata. Staeg. phantasma. R.-Desv.

Themira. Mcq.

minor. Hal.

lucida. Staeg.

Fallenii. Staeg. cylindrica. Fll.

Leachii. Ztt. ol. pilosa. R.-Dev. superba. Hal. pusilla. Ztt. albitarsis. Ztt. gracilis. Ztt. 396. Nemopoda. R.Desv. - Musca. F. - Calobata. F. Sepsis, Auct. cylindrica. F. nitidula. Fll. mutris. R.-Desv. 
stercoraria. R.-Des.

varipes. $\mathrm{Mg}$.

nigricormis. Mg. nigrilatera. Meq. tarsalis. Wlk.

fumipennis. Wlk.

viridis. Mreq.

397. Se pis. Fll. - Mresca. L. F. - Thephritis. F. - Micropeza. Ltr. - Enicita. Wstw. - Enicopus. Wlk.

\section{Enicita. Westw.}

amnulipes. Mg.

$$
\text { Sepsis. Fll. }
$$

punctum. F. stiyma. $\mathrm{Pz}$. cynipsea. R.-Desy. violacea. $\mathrm{Hg}$. pyymaea. R.-Desv. cynipsea. L.

fulgens. Mg. hilaris. $\mathrm{Mg}$. ruficornis. Mg. fulyida. R.-Desr. vivida. R.-Desv. flavimana. $\mathrm{Mg}$. rufipes. Mg. cornuta. Mg. nigripes. Mg. nitida. R.-Desv. barbipes. Mg. ornata. Mg. atripes. R.-Desv. pectoralis. Meq. maculipes. Wlk. concimna. Wlk. duplicata. Hal.
Cephalinae.

398. Cephalia. Mg. Myrmeromyia. R.Desv.

nigripes. $\mathrm{Mg}$. rufipes. Mg.

formicaria. R.-Desv. quadripunctata. Gimm.

\section{Trypetinae.}

$D$ acinae.

399. Dacus. Mg. Muscr. F. - Osnis. F. - Brachyopa. Mg. ol. oleae. F.

400. Ceratitis. Mac. Leay. - Tephritis. W. - Petalophora. Meq.

hispanica. Bréme.

? capitata. W.

$$
\text { Trypetinae. }
$$

401. Anomoia. Wlk. Trupeta. W. - Anomoea. Lw.

antica. IV.

Gaedii. Mg.

Zetterstedtii. Boh. 402. Euphranta. Lw。 - Musca. F. Dictya. F. - Ortalis. Fll. - Mosina. R.Desv. - Cordylura. Meq. - Tephritis. Heq. comnexa. F.

Zetterstedtii. Fll. dorsalis. Meq.
403. Platyparea. Lw. - Musca. F. - Ortalis. $\mathrm{Mg}$. pt. - $\mathrm{Te}-$ phritis. Fll. - Trypeta. Mg. - Urophora. Meq. - Aciura. Wlk. - Poeciloptera. Lw. ol.

discoidea. F.

lychnidis. Fll. Mg. caloptera. Lw. poeciloptera. Schrk. fulminans. Mg.

404. A ciura. R.-Desv. Trypeta u. Tephritis. Auct. - Urophora. Meq.

femoralis. R.-Desr. rotundiventris. $\mathrm{Mg}$. ral。

tibialis, R,-Desv. gagates. Lw. ol. rotundiventris. Fll. Wimnertzii. Ffld. 403. H e milea. Lw. Trypeta. Costa. dimidiata. Costa. 406. A cidia. R.-Desv. Musca. L. F. Scatophaga. F. Trupeta u. Tephritis. Auct. - Aciura u. Euleia. WVlk. - Urophora. Mcq. - Epidesmia und Myoleja. Rond.

heraclei. L. onopordinis. F. Mg: centariene. F. berberidis. Ell. lucida. Fll. cognata. $\mathrm{Mg}$. speciosa. Lw*. 
cognata. W.

Iychnidis. F.

rentaureae. Fll.

discoidea. $\mathbf{M g}$.

flarescens. F.

marmorea. $\mathrm{F}$.

407. Spilog a pha I,w. - Musca. L. F.

- Trupanea. Schrk.

- Trypeta u. Tephritis. Auct. - Zonosema u. Rluagdetis. I, w.

Spilographa. Lw.

Zoë. $11 \mathrm{~g}$.

apicalis. Ztt.

abrotani. $M \mathrm{Ls}$.

lamifera. Lw.

artemisiae. $\mathrm{F}$.

interrupta. Fll.

onopordi. R,-Desp.

intermissa. Mg.

Rhagolet $\{s . \mathbf{L w}$

cerasi. I

solstitialis. Sulz.

signata. MI.

liturata. R.-Desr.

Zonosema. Lw.

alternata. Fll.

continua. $M g$.

Meigenii. Lw.

alternata. Mg.

408. Hy p en idium. Lw. graecum. Lw.

409. Oedaspis. Lw. -

Timpeta. Lw. ol. multifasciata. $\mathrm{T}, \mathrm{w}$.

fissa. L,
410. Orellia. R.-D sr.| lappae, Cederiyj. - Trupeta u. Tephritis. Auct. - Oedaspis. Lw. pt.

Wiedemannii. $\mathbf{M g}$. flavicans. R.-Dest.

Schineri. Lw.

411. Trypeta. $\mathbf{~ I g . ~ - ~}$ Musca. L. Scop. F. - Scatophaya, Dacus. F. - Trupaneu. Schrk. - Tephritis. F11. Ztt. - Sitarea u. Terellia. R.-Desv. Carpomyia, Cerajocerat. Rond.

jaceae. R.-Desv.

dorsalis. R.-Desr.

pusilla. R.-Dest.

aretii. Meq.

punctate. Lw. ol. falcata. Scop.

lappae. $\mathrm{Mg}$. scorsonerae. R.-I)es. octopunctata. Meq. succinea. Costa. intermedia. Efld. lurida. I,w. stigmataspis. 11. onotrophes: Lw. solstitialis. Pz. arctii. Mg. cylindrica. R.-Desv. Lw.

dorsulis. Mcq. hexacheta. Lw. cornata. F. distans. Lw. tussilaginis. F. arctii. Deg. Lw. acanthi. Schrk. fuscicornis. Lw. tussilaginis. Wik. colon. Mg.

Wenizeri. $\mathrm{Mg}$. dispar. Ztt. abrotani. Meq. Alciphron. Newm. nigricoma. Lw nebulosa. Mcq. ruficauda. F.

Horescentiae. Mg: Lw. punctata. Fll.

Winthemi. Mg. acuticornis. Lw. longicauda. Mg. dentata. Lw. servatulae. $\mathrm{Mg}$. palpata. R.-Desv. pallens. Mcq. serratulae. L. pallens. Mg. luteola. R.-Desv. virens. L $\mathrm{Lw}$.

obscura. Brullé. tanaceti. Schrk. Hebe. Newm. plagiata. Dahlb. immaculata. Meq. 412. Ur o phora. R,-Dsv. - Musca. L. F. Dacus. F. - Trupanea. Schrk. Scatophaga Germ. Tephritis. Fll. Ztt. Trypeta. Mg. I.w. ol. stigma. Lw.

unimaculata. v. Ros. manra. Ffld. 
solstitialis. L.

dauci. F.

hastata. F.

leucacanthi. Schrk.

pugionata. Mg.

cuspidata. Meq.

macrura. Lw.

cuspidata. $\mathbf{M g}$.

terebrans. IAw.

stylata. F.

jacobeae. Pz.

irsii. Schrk.

atfinis. Ftld.

congrua. Lw.

cardui. L.

flexuosa. Germ. quadrifasciata. $\mathrm{Mg}$.

Dejeani. R.-Desf.

centaureae. R.-Dess.

eriolepidis. Lw.

aprica. Fll.

solstitialis. $\mathrm{F}$.

femoralis. R.-Desv. placida. Müll.

spoliata. Hal.

413. M y opites. Brébiss.

- Trypeta. Mg. -

Ensina. Mcq. -.. Sty-

lia. R.-Desv.

inulae. $\mathrm{V}$. Ros.

stylata. F.

Frauenfeldi. Schin.

Blotii. Ffld.

limbardàe. Schin.

Blotii. Brébiss.

longirostris. Lw.

tenella. Ffld.

maculata. R-Desv.

mentharum. R.-Desr.

jasoniae. L. Duf.
414. Ensina. R,-Desr. - Musca. I. - Trypeta. Mg. - Tephritis. $7 \mathrm{tt}$.

sonchi. L.

obsoleta. $\mathrm{MIg}$.

chrysanthemi. R.-Dsv.

herluarum. R.-Desv.

pratensis, $\mathrm{R},-\mathrm{Des}$.

linariae. R.-Desv.

scorzonerae. R.-Desr.

doronici. R--Desv.

asteris. Hal.

415. Rhacochlaena. Lw.

toxoneura. Lw.

\{16. T ep hr it is, Ltr. -

Musca. L. F. - Dacus. F. - Trupanea.

Schrk. - Trypeta.

Mg. - Acinia. Meq.

- Sphenella, Oxyna,

Tephritis u. Urellia.

R-Dest. u. Lw.

Splenella. Lw.

marginata. Fll.

inuriae. R.-Desv.

arcuata. Schrk.

Urellia. Lw.

eluta. Mg.

cometa. Lw.

radiata. Fll.

ramulosa. Lw.

stellata. Fuessli.

radiata. F.

terminata. Fll.

calcitrapae. R.-Desv.

parisiensis. R.-Desr.

amoena. Ffld.

gnaphalii. Lw.

discoidea. Ell.

aestiva. Mg.
Mamulae. Ffld.

teminata. $\mathrm{Mg}$.

filaginis. Lw.

Tephritis. Lw.

truncata. Lw.

formosa. Lw.

arnicae. L.

Havicauda. Mg.

Neesii. v. Ros.

arnicivora. Lw.

Egereri. Ffld.

ainicae. Lw. pt.

conura. Lw.

flavicauda. Ztt.

matricariae. Lw.

praecox. Lw.

ruralis. Jw.

fallax. Lw.

leontodontis. Fll.

vespertina. Lw.

cincta. Lw.

dioscurea. Lw.

leontodontis. Deg.

hyoscyami. Fll.

millefolii. R.-Desv.

dilacerata. Lw.

bardanae. Schrk.

confusa. Mg.

heraclei. TVlk.

plantaris. M,-Desv.

stictica. Lw.

segregata. Fild.

hyoscyami. L.

postica. Lw.

heraclei. F. Mg.

valida. Lw.

nigricauda. Lw.

angustipennis. Lw.

leontodontis, Ztt.

conjuncta. Lw.

pulclira. Lw.

megacephala. Iw. 
Zelleri. Lw. irrorata. Fll. guttata. Fll. gemmata. $\mathrm{Mg}$. Oxyna. Lw. pantherina. Fll. parietina. F. Mg. cinera. R.-Dest. flavipennis. Lw. flavescens. R.-Desv. laticauda. Wlk. pyrethri. R.-Desv. proboscidea. Lw. obesa. Lw. plantaginis. Hal. parrula. Lw. tessellata. Lw. argyrocephala. Lw. doronici. Lw. absinthii. F.

punctella. Fll. pt. stenoptera. Lw. producta. Lw. elongatula. Lw. tenera. Lw.

fenestrata. Ztt. laticauda. Mg. Theora. Newm. Alethe. Newm.

417. Ox y phora. R.-Dsv. - Musca. F. Schrk. - Trypeta. Mg. Tephritis. Fll. Ztt. Acinia, Xiphosia und Oxyphora. R. - Desv. - Forellia. Wlk. miliaria. Schrk. arcuata. F. Pz. sphaerocephali. Schk. arnicae. Fll. $\mathrm{Mg}$. cijsionum. R.-Desv. Hava. Is.
Sclineideri. Lw.

biflexa. Lw.

corniculata. Ztt.

biarcuata. Curtis.

Westermannii. $\mathbf{M g}$. cardui. R.-Desr.

Schefferi. Ffld.

418. Carphotricha.

Lw. - Trupanea. Schrk. - Trypeta u. Tephritis. Auct. Acinia.Meq. - Noeeta. R.-Desr. - Dithryca u. Oplocheta. Rond.

guttularis. Mg.

capitata. Fll.

flavipes. R.-Desv.

brunnicosa. R.-Desv. pupillata. Fll.

reticulata. Schrk. strigilata. Lw.

Sapromyzinae.

419. Pallo ptera. Fll. Musca. L. F. - Sapromyza. Mg. Suillia. R.-Desv. ustulata. Fll. umbellatarum. F. gangraenosa. Fll. usta. $\mathrm{Mg}$. scutellata. Meq. saltuum. I.

unicolor. $\mathrm{F}$.

marginella. Fll. arcuata. Fll. ambusta. Mg. quinquemaculata. Mcq. quinquepunctata. $\mathbf{M g}$. trimacula. Mg.

trimaculata. Ztt. ephippium. Zitt.
4.0. Peplomyza. Hal. - Sapromyza. Mg. - Opomyza. Mg. - Phyllomyza. Hal. ol.

Baumhaneri. Lw. litura. Mg: pt. discoidea. Mg.

Wiedemanni. Lw. litura. $\mathbf{M g}$. pt. 421. S a p r o m y za. Fll. Musca. I. F. - Tephritis. F. - Lauxania u. Sapromyza. Mg. - Minettia, Sylvia, Lycia, Terenia u. Herbina. R.-Desv.

-Toxoneura. Mcq.pt. longipennis. F.

lnctuosa. R.-Dess. nigripes. Mcq. basalis. Ztt, bivittata. Mg: Loewii. Schin. bicolor. Lw. albitarsis. Mg: lupulina. F. pallida. $\mathrm{Mg}$. dimidiata. Lw. subfasciata. 7tt. bicolor. Meq. grisea. $\mathbf{M g}$. palustris. R-Desv. flaviventris. Costa. fasciata. Fll. rivosa. $\mathrm{Mg}$. nemorosa: $\mathrm{R}_{4}$-Desv. subvittata. Lw. plumicornis. Fll. decempunctata. Fll. multipunetata. FH. 
notata. Fill.

duodecimpunctata. IIcq.

sexnotata. Ztt. notata. Mcq. novempunctata. Gimm. pallidiventris. Fll.

pallidicomis. Lw.

bipunctata. Mg. quadripunctata. F. tibialis. Mcq. senilis. Mg. sexpunctata. Mg. quadripunctata. Schrk.

tarsella. Ztt. obsoleta. Lw. obsoleta. Fll. apicalis. R.-Desv. anysodactyla. Lw. spinitarsis. Ztt. simplex. Lw. setiventris. Ztt. pallida. Mg. apicalis. Lw. obsoleta. Mg. pallida. Fll. illota. Lw. praeusta. Fll. pt. affinis. Ztt. decipiens. Lw. sordida. Hal.

laeta. Ztt. rorida. Fll. flava. K.-Desv. brumitarsis. Meq. platycephala. Lw. praeusta. Fll.

litus'ata. R.-Desv. obseuripemis. Lw.

dubia. Mcr. rubetra. R.-Desv. marginata. $\mathrm{Mg}$. inusta. Mg:. albiceps. Fll. albifrons. Mcq. placida. MIg. tenera. Lw. decaspila. Lw. modesta. Lw. nana. Lw. biumbrata. Lw. difformis. Lw. tubifer, MIg. nigrimana. $\mathrm{Mg}$. costata. Mg. Roberti. Mg. punctata. Mcq. suillorum. R.-Desv. grossipes. R.-Desv. fuscicornis. Meq. binotata. Mcq. dorsalis. Meq. hilarella. Ztt. obesa. Ztt. melanogaster. Ztt. chlorophthalma. Ztt. fuscotestacea. Ztt. quadrinotata. Ztt. melanura. Ztt. basalis. Ztt. flavipalpis. Lw. biseriata. Lw. septentrionalis. Lw. intonsa. Lw. longiseta. Lw. maculipemis. Lw. quadrivittata. Lw. citrina. $\mathrm{Mlg}$. dubia. Gimmerth. sexmaculata. Gimm.

Toxoneura. Mcq. fasciata. Nicq.
422. La uxa nia. Ltr. - Musca. F. - Dolichopus u. Sargus. F. - Calliope. Hal. pt.

cylindricornis. F. cle'ysoptera. Schrk rufitarsis. Ltr. Elisae. Mg. atrimana. $\mathbf{M g}$. aenea. Fll. nitens. Lw. atripes, Mg. frontalis. Lw. glabrata. F. geniculata. F. vitripennis. Mg. hyalinata.. Mg. scutellata. Mg. leucostoma. Ztt. amica, Hal.

423. P a ch y cerina. Meq. Lauxania. Mg. Fll. seticornis. Fll. signatipes Lw.

424. Cnemacantha. Mcq. - Heteroneura. Fil. - Agromyza. $\mathrm{Hg}$.

muscaria. Fll.

425. Lonchaea. Fll. Musca. F. - Lonchaea und Teremyia. Veq. - Lonchaea u. Earomyia. Ztt. Lasiops. Mg. pt. Chortophila. Meq. pt. hirticeps. Ztt. dasyops. Mg. lasiophthalma. Meq. aenescens. $\mathrm{Mg}$. fumosa. Egg. 
Deutschii. Ztt.

aenea. Mg.

vaginalis. Fll.

chorea. F.

palposa. Ztt.

tarsata. Fll.

parvicornis. Mg.

laticornis. Mg.

pusilla. Mig.

latifrons. $\mathbf{M g}$.

leucostoma. M $\mathrm{g}$.

pumila Mg.

nigra. $M \mathrm{~g}^{\circ}$.

viridana. $M g$.

nigrimana. $\mathrm{Mg}$.

ensifer. Mg.

crepidaria: Mg:

carbonaria. Mg.

nigella. $\mathbf{M g}$.

nigritarsis. Mcq.

crassinervis. Ztt.

parvula. Ztt.

flavidipenuis. Ztt. pallipennis. Ztt. hyalipennis. Ztt. albitarsis. Ztt. metallica. Ztt.

Earomyia. Ztt.

lonchaeoides. Ztt.

Ulidinae.

426. Chloria. Schin. -

Musca. F. - Tephritis. F. - Ulidia. Mg. - Chrysomyza. Fll.

demandata. F.

splenáida. Fll.

bicolor. Meq.

427. Ulidia. $\mathbf{M g}$.

erythrophthalma. Mg. nigripennis. Lw.

nitida. Mg.

nitens. Lw.

parallela. Lw.

428. Timia. W. - Ulidia.

Lw. pt.

apicalis. $\mathbf{M g}$.

erythrocephala. IV.

megacephala. Lw.

429. M y o din a. R.-Desr.

Musca. L. F. - Te-

phritis. F. - Sciopte-

ra.Kirby. - Ortalis.

Fll. Mg.

vibrans. $L$.

urticae. R.-Desv.

Platystominae.

430. Platystoma. Mg.

- Dictya. F. -

Muscu. F. - Tru-

panea. Schrk. -

Hesyquillia. R.-Desv.

seminationis. $F$.

tegularia. Lw.

umbrarum. F.

fulviventris. Gmel.

lugubis. R.-Desv.

transversa. Itr.

pubescens. Lw.

angustipennis. Lw.

rufipes. Mg:

bifasciata. Brullé.

subfasciata. Lw.

$$
\text { Ortalinae. }
$$

431. Ps a i o p te ra. Whlbg. - Homalo- 434. Myenuis. R.-Desr. cephala. Ztt.

albitarsis. $7 \mathrm{tt}$.

biumbrata. IVhlbg. angustata. Whlbg: apicalis. Ithlbg. bimaculata. Whlbg.

bipunctata. Lw.

432. Herina. R.-Desv.

- Musca. L. F. ...

Tephritis. F. - O

talis. Auct.

frondescentiae. L.

cerasi. $\mathrm{F}$. Mg.

mali. F.

morio. F.

uliginosa. Fll.

luctuosa. MIg.

moerens. Mg.

nigrina. $\mathrm{Mg}$.

limbata. R.-Desv.

liturata. R.-Desv.

afflicta. Mg.

lugens. Mg.

lugubris. Mg.

lacustris. $\mathrm{Mg}$.

paludum. Fll.

longicomis. R.-Desv.

palustris. Mg.

oscillans. Mg. Schin.

parva. Lw.

apicalis. Ztt.

rufitarsis. Meq.

scutellaris. R.-Desv.

gylans. Lw.

433. Rivellia. R.-Desr.

- Musia. F. - TC-

phritis. F. - Ortalis.

Auct.

syngenesiae. F.

juncorum. Ell.

urticae. Schrk.

herbarum. R.-Desv.

- Scatophaya. F.-

Tephritis, H. - Trypeta. $M g \cdot p t$ - $\mathrm{Or}^{\circ}$

talis. Mg. pt. 
fasciata. $\mathrm{F}$.

rivularis. $\mathrm{F}$.

435. Ceroxys. Meq. -

Musca. L. E. - Sca-

tophaga. F. - Orta-

lis. Auct. - Melieria

u. Meclielia. R.-Desv.

urticae. L.

cinifera. Lw.

acuticornis. Lw.

omissa. Mg.

ganyraenosa. Pz.

crassipennis. F.

gangraenosa. R.-Dsv.

albipennis. Lw.

hyalinata. Pz.

marmorea. F. Mg.

elegans. R.-Desr.

fraudulosa. Lw.

unicolor. Lw.

picta. $M g$.

flaroscutellata. Meq. pulicaria. R.-Desv. quinquemaculata. Meq.

quinquenotata. Mg. 436. Tetanops. Fll.

myopina. Hll.

impuncta. Lw.

flarescens. Meq.

psammophila. Lw.

437. Ortalis. Fll. -

Musca. Pz. - Dic-

tya, Scatophaya, Te-

phritis. F. - Oscinis.

Ltr. - Blainvillia.

R.-Desv.

centralis. F.

Friesii. Fll.

guttata. Mg.

nebulosa. Mg.

murina. Lw。 formosa. $\mathrm{Pz}$.

yangraenosa. $\mathrm{E}$. ornata. Mg.

gangraenosa. Mg.

grata. Lw.

ruficeps. F.

tristis. Mg.

latifrons. Lw.

angustata. Lw.

jucumda. R.-Desv.

maculipennis. Ltr.

rufipes. Meq.

bivittata. Mcq.

438. O tit es. Ltr. - Mus-

ca. F. - Scatophaya.

F. - Ortalis. Mg.

pt. - Sciomyza. Mg.

pt. - Heramyia. R.-

1)esv.

lamed. Schrk.

pulchella. F. Mg.

lobata. F.

bucephala. Mg.

Dorycerinae.

439. Dorycera. Mg. Musca. F. - Scatophaga. F. - Tetanocera. Ltr.

graminum. $\mathrm{F}$.

hybrida. Lw.

inornata. Lw.

440. Trigon o m etopus.

Meq. - Tetanocera.

Mg. ol. - Oxyrhina.

Mg:

frontalis. $\mathbf{M g}$.

441. Pyrgota. W. -

- Adapsilia. Waga.

coarctata. Waga.
Ayramyzinae.

4\{. Phytomyza. Fll. Chromatomyia. Hardy. - Napomyza. Hal. pt.

elegans. Mg.

festiva. $\mathrm{Mg}$.

Iateralis. Fll.

sonchi. R.-Desv.

anuulipes. Mg.

flara. Fll.

ranunculi. R.-Desy.

scolopendrii. R.-Dsv

analis. Ztt.

pallida. Mg.

terminalis. Mg.

nigripeunis. Fll.

albipennis. Fll.

obscurella. Fll.

flavicornis. Fll.

flaroscutellata. Fll.

scutellata. Mg.

incisa. Meq.

Zetterstedtii. Schin.

maculipes. Ztt.

praecox. Mg.

pullula. Ztt.

geniculata. Mcq.

horticola. Gour.

affinis. $\mathrm{Mg}$.

nigricomis. Mcr.

albiceps. Mg:

syngenesiae. Hardy.

rufipes. Mg.

sulphuripes. Mg.

nitidicollis. Mg.

heteroptera. Lw.

tridentata. I w

gymnostoma. Lw.

bipunetata. Isw.

acuticornis. Lw. 
xanthaspis. IN. oscinina. Fll. marginella. Fll. nigra. MIg.

sphondyli. R.-Desv. atra. Mg. agromyzina. Mg. notata. $M \mathrm{~g}$. minima. $11 \mathrm{~g}$. fasciata. Mg. temella. Mg. viduata. $\mathrm{Mg}$. vitripennis. $\mathrm{Mg}$. atricornis. $M \mathrm{~g}$. stylata. Mg. confinis. $\mathrm{Mg}$. nigritella. Ztt. morio. Ztt.

abdominalis. Ztt.

maryinella. Fll. pt. pygmaea. Ztt. ruficornis. Ztt. pallicornis. Ztt. flaviventris. $/ \mathrm{ttt}$. fuseula. Ztt. nigritula. Ztt. ol. atomaria. Ztt. zonata. Ztt. crassiseta. 'Ztt. flaviceps. Mcq. flavipes. Mcq. nigricans. Meq. fasciata. Heq. varipes. Meq. femoralis. Ztt. ol. geniculata. Brullé. Jiturata. Brullé. maculipes. Brullé. xylostei. R,-Desv. plantaginis. R.-Desv. lappina. R.-Dest. cinerella. R.-Desv. aquifolii. Gourrean. ancholiae. 1R.-Desv. aquilegiae. Hardy. cinereofrons. Hardy. populicola. WVlk. artemisiae. Kaltb. chaerophylli. KaItb. corni. Kaltb.

443. A grom yza. Fll. Iacteipennis. Fll. vagans. 1ll. pectinata. Mg. obscurella. Fll. infuscata. $\mathrm{Mg}$. lutea. $\mathbf{M g}$. flava. Mg. pusilla. $\mathrm{Mg}$. abiens. Ztt. flavifrons. Mg. capitata. Ztt. geniculate. $\mathrm{Mg}$. geniculata. Fll. reptans. Fll. mobilis. Mg. rufipes. $\mathrm{Mg}$. nigripes. Mg. ambigua. Ztt. ol. gyrans. Fll. errrans. Mg: carbonaria. Ztt. nicjra. Ztt. ol. posticata. Mg. aeneoventris. Fll. aenea. $\mathrm{Hg}$. M.-atrum. Mg. pulicaria. $\mathrm{Mg}$. cunctans. $\mathbf{M g}$. Schineri. Giraud. curvipalpis. Ztt. maura. Mg. pulicaria. Ztt. ol. morionella. Ztt. pinguis. Fll. sordida. Fll. scutellata. Fll.

flaveola. Fll. pt. gracilis. $\mathrm{Mg}$. nigritarsis. Mg. strigata. $\mathrm{Mg}$. amoena. Mg. hyalipemis. Mg. signata. Mg. pallida. Mg. imbuta. Mg. minuta. $\mathrm{Mg}$. basilaris. $\mathbf{M g}$. albicornis, Mg. pascuum. Mg. variegata. Mg. fasciola. Mg. occulta. Mg. vittata. Mg. lateralis. Mcq. rerbasci. Bouché. thapsi. Bouché. holosericen. Bouché. heraclei. Bouché. lappae. Lw. tibialis. Fll. flaveola. Fll. flaviceps. Fll. nana. Mg. ircos. R.-Dest. morosa. Mg. albipennis. Mg. albitarsis. Mg. bimaculata. Mg. anthracina. Mg. leucocephala. Mg. fulvipes. MIg. frontalis. Mg. ormata. $\mathrm{Mg}$. muscina. Mg: atra. $\mathrm{Mg}^{\circ}$. pulla. Mg. pygmaea. $\mathbf{M g}$. perpusilla. $\mathbf{M g}$. 
luctuosa. Mg. incisa. $\mathrm{Mg}$. exigua, $\mathrm{Mg}$. annulipes. Mg. pumila. Mg. exilis. Mg. orbona. $\mathrm{Mg}$. pusio. Mg. puella. Mg. blanda. Mg. calceata. Mg. bicolor. $\mathrm{Mg}$. atricornis. Mg. nigrimana. Mg. fuscipes. MIcq. varipes. Meq. pallitarsis. Meq. ruficornis. Heq. ruficeps. Mcq. obscuripennis. Mcq. cinerascens. Mcq. pallipes. Mcq. rufifrons. Meq. rufimana. Meq. thoracica. Meq. laterella. Ztt. lineella. Ztt. albitarsis. Ztt. flavicornis. Ztt. coxata. Ztt. niveipennis. $\mathrm{Ztt}$. aenescens. Ztt. albohyalinata. Zitt. breviseta. Ztt. M.-nigrum. Ztt. elegantula. Ztt. variceps. Ztt.

haemorrhoidalis. Ztt. cingulata. Ztt. limbatella. Ztt. superciliosa. Ztt. carbonella. Ztt. rittigera. Ztt. grossicornis. Ztt. xanthocephala. Ztt. tarsella. Ztt. flarimana. Ztt. flavoscutellaris. Ztt. scutellata. Fll. pt. minutissima. Ztt. atripes. Ztt. virgo. $Z$ tt. bilarella. Ztt. elongella. Ztt. pistaciae. Curtis. violae. Curtis. Macquarti. R.-Desv. flavonotata. Hal. arctii. Kaltb. latipes. $\mathrm{Mg}$.

44. C e r a t o m y za. Schin. - Ayromyza. Auct. - Chlorops. Fll. pt. - Odontocera. Meq.

flavicornis. Egg. denticornis. Pz.

Meigenii. Fll. acuticornis. Hg. continis. $\mathrm{Mg}$. femoralis, Mg. affinis. Fll. spinicornis. Meq. lateralis, Ztt. tarsata. $\mathrm{Mg}$. 445. Leiomyza. Mfcq. - Agromyza. Mg. Heteroneur. Fll. laevigata. $\mathrm{Mg}$. scatophagina. Ell.

Alavipes. Fll.

curvipennis. Ztt. ol. glabricula. $\mathbf{M g}$.
Milichinae.

\{́6. Phyllomyza. Fll. - Ayromiza und Opomyza. Mg. pt. securicornis. Fll.

? flavitarsis. Mg. flaritarsis. $\mathbf{M g}$.

447. Lobioptera. Whibg. - Milichia. Mg. pt. - Argyrites. Ltr. speciosa. Mg. palposa. Ztt. ludens. Whlbg.

448. Milichia. Mg. Odinia. R.-Desv. boletina. Ztt. maculata. Mg. trinotata. R.-Desv. formosa. Lw. pulchra. Ztt. ornata. Ztt. tarsata. Ztt. 449. Ca coxenus. Lw. indagator. Lw.

Ochthiphilinae.

450 Leucopis. Mg. Anthomyza. Fll. Agromyza. Ztt. ol. griseola. Fll. lusaria. Mg. punetella. Z $\mathrm{Zt}$. puncticornis. Mg. annulipes. Ztt. griseola. Mg. silesiaca. Egg。. nigricornis. Egg. argentata. Heeg. albipennis. Mg. nudiceps. Ztt. geniculata. $Z t t$. 
albipunctata. Ztt. argenticollis. Ztt. cinerella. Ztt. hyalipemis. Ztt. tibialis. Ztt. obscura. Hal. magnicornis. LW. 431. Ochthiphila. Fll.

- Chamaemyia. Pz.

- Estelia. R.-Desv. spectabilis. Lw. coronata. Lw. elegans. Pz. maritima. 7tt. polystigma. $\mathrm{Mg}$. geniculata. Ztt. juncorum. Fll.

herbarum. R.-Desv. midella. Fll. fasciata. Lw. nigrimana. lig. albiceps. Mg. flaripes. Mcq. nigripes. MIcq. geniculata. Hal. nigricornis. Perris.

4.5. A c rometopia. Schin. - Oxyrhina. Vtt.

Wahlbergi. Ztt.

Heteroneurinae.

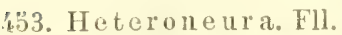
albimana. $\mathrm{Mg}$. pictipes. Ztt. alpina. Lw. ruficollis. Mg. laterella. Zitt. nubila. Mg. geomyzina. Fll. muficollis, Ztt. 4ơ. Clusia. Hal. - Heteroneura. $\mathrm{Mg}$. Lw. - Macrochira. Ztt. - Amplipogon. Whlbg. - Therina. Ztt. Clusia. Hal.

flava. Mg.

spurca. Hal. decora. I,w.

Amplipogon. Whlbg.

spectrum. Whlog.

flava. Ztt. ol.

Cordylurinae.

\&.3̆. I eptopa. Ztt. Cordylura, Fll. Hal. pt. - Delina. R.Desv.

filiformis. Ztt. pallida. Fll, pt. Haveola. Ztt. ol. flava. Hal.

456. Hy dromyza. Fll. - Musca. k. - Covdylu: ${ }^{\circ}$. Auctor. Nupharia. R.-Desv. Fallenii. Schin. hydromyzina. Fll. fraterna. $\mathbf{M g}$. livens. Fll.

Kunzei. 'Ztt. albitarsis. Ztt.

457. Cleigastra. Heq. - Coriylura. Auct. macrocera. Mg. apicalis. Mg. punctipes. $\mathrm{Mg}$. Havipes. Mg. Friesii. Ztt. flavipes. Fll. brevifions. Ztt. ustulata. Zit. anthrax. Schin. fasciata. Mig. pilosa. Ztt. tibiella. Ztt. haemorrhoidalis. Mg. erythrocephala. Mg. punctum. Mg. fulvipes. $\mathrm{Mg}$. bicolor. Meq. frontalis. Meq. albipenuis. Ztt. cuneiventris. Ztt. albipila. Ztt. nigripila. Ztt. breviseta. Ztt.

maynicornis. Ztt. nigripes. Ztt. proboscidea. Ztt. strigifrous. Ztt. nigrita. Fll. loxocerata. Ell. lonyifrons. Ztt. aricina. $/ \mathrm{tt}$. aricioides. Zut. nigrimana. Ztt. clavata. Ztt. lividipes. Ztt. olscurella. Ztt. niveipalpis. Ztt. pallipes. Ztt. melanura. Ztt. ol. lineata. Ztt. fulvipes. Ztt. pallicicauda. Ztt. planiceps. Ztt. leucostoma. Ztt. obscur:t. Fll. armillata. Ztt. cinerella. Ztt. immunda. Ztt. dorsata. Ztt. pectoralis. Ztt. ol. 
abdominalis. Ztt. latipalpis. Mg. tarsea. Fll.

nigriventris. Lw. glaucescens. Lw.

458. Pogonota. Ztt. Cordylura. Ztt. ol. caudata. Ztt. melanura. Ztt. barbata. Ztt. crassipes. Ztt. hircus. Ztt. sponsa. Ztt. dasyprocta. Lw. 459. Norellia. R.-Desv. - Cordylura. Auct. nervosa. Mg. armipes. $\mathrm{Mg}$. striolata. $\mathrm{Mg}$. alpestris. Schin. spinimana. $\mathrm{Mg}$. semiflava. $\mathrm{Pz}$. spinipes. MIg. pseudonarcissi.R.-Dv. liturata. $\mathrm{Mg}$. spiniger $a$. Ztt. flavicormis. Mg. flavicauda. Mg. trilineata. $\mathrm{Mg}$. ruficauda. Ztt. dentimana. Ztt. Zetterstedtii. Gimm. 460. Cordy lura. Fli. Musca. L. - Ocyptera. F. - Mosina. R.-Desv.

pubera. L. pudica. Mg. ciliata. Mg. tibialis. Ztt. incerta. Ztt. rufimana. Mg. lurida. Schin. albilabris. F. unicolor. Lw. albipes. Fll. filipes. R.-Desv. bilineata. Mg. picipes. $\mathrm{Mg}$. rufipes. Mg. pallida. Fll. geniculata. Ztt. atrata. Ztt. fuscipes. Ztt. unilineata. Ztt. scatomyzoides. Ztt. dispar. Ztt. incisa. $\mathrm{Mg}$. nigra. R.-Desv. Dejeani. R.-Desv. albofasciata. Gimm. fuscipennis. Gimmerth. marginipennis. Gimm. brevipennis. Curtis. biseta. Lw. opaca. Lw, femoralis. Lw. vittata. Mg. filiformis. Ztt.

2. Muscidae calypterae.

Anthomyzinae. 461. Sylle go ptera. Rond. - Anthomyia u. Coenosia. Mg. ocypterata. $\mathrm{Mg}$. 462. Coenosia. $\mathrm{Mg}$. Musca. Deg. F. Fll. - Aricia u. Anthomyza. Ztt. - Limosia, Palusia u. Caricaea. R. - Desr. Coenosia, Chelisia, Oplogaster u. Mycophaga. Rond. Schoenomyza. Hal. pt. fungorum. Deg.

alma. Mg.

spuria. Ztt. ol. tigrina. $\mathrm{F}$. ciliatocosta. Ztt. tigrina. Mg. exul. Ztt. pacifica. Mg. triangula. Fll.

pictipennis. Lw. mollicula. Fll.

nemoralis. $\mathrm{Mg}$. tricolor. Ztt. biocellata. Ztt. geniculata. Fll. sexnotata. Mg. sexmaculata. Mg. pumila. Fll.

albicornis. $\mathrm{Mg}$.

testacea. R.-Desr. albicornis. $\mathrm{Mg}$. intermedia. Fll. means. $\boldsymbol{M g}$. paludosa. R.-Desv. nigrimana. Mg. nigrifemur. Ztt. meditata. Fll. verna. F. monilis. $\mathrm{Mg}$. umbripennis. Ztt. remotella. Ztt. humilis. Mg. decipiens. Mg: rivularis. R.-Desv. nana. Ztt. pedella. Fll. vaccarum. Bouché. murina. Mg. nigra. Mg. luctuosa. Mg. 
ambulans. Mg. minima. $\mathrm{Mg}$. albipalpis. Mg. perpusilla. Mg. punctipes. $\mathrm{Mg}$. simplex. Mg. rufipalpis. Mg. atra. $\mathbf{M g}$.

peregrina. Mg. incompta. Mg. agromyzina. Fll. obtusipennis. Fll. tuberculiventris. Ztt. solitaria. Ztt. maculigera. Ztt. articulata. Ztt. albifrons. Ztt. Trollii. Ztt. albitarsis. Ztt. albimana. Ztt. troglodytes. Ztt. vilis. Ztt. albatella. Ztt. flavicornis. Ell. bilineella. Ztt. cillgulipes. Ztt. pygmaea. Ztt. lacteipennis. Ztt. lamellata. Ztt. trilincella. Ztt. fulvicornis. Ztt. flavimana. Ztt. octopunctata. 'Ztt. strigella. Ztt. ol. minutalis. Ztt. pulicaria. Ztt. antennata. Ztt. metallipennis. Ztt. graminum. Ztt. pallicornis. Ztt. rubricornis, Ztt. rufina. Fll.

ochreata. Ztt.ol. tarsella. Zitt. dealbata. Ztt. litoralis. Ztt. albimana. Lw. dubia. Meq . ammulata. Mcq. ruficormis. Mcq. basilaris. Mcq. testacea. Meq. rulgaris. R.-Desr. lappae. R.-Desv. erythrocera. R.-Desw. leucophaea. R.-Desv. hydrocotylis. R.-Desv. nigripes. R.-Desv. palustris. R.-Desv. menyanthides. R.-DsF. abdominalis. Gimm. testacea. Gimmerth.

Schoenomy 2 .

litorella. Fll. fasciata. Mg.

albiceps. Mg. facilis. $\mathrm{Mg}$.

463. A the r ig on a. Rond. - Anthomyia. Mg. raria. Mg.

464. L is pe. Ltr. - Lispa. Ltr. - Musca. Deg: crassiuscula. Lw. litorea. Fll. monacha. Schin. tentaculata. Deg. fluviatilis. R.-Desp. consanguinea. Lw. melaleuca. Lw. uliginosa. Fll. hydromyzina. Fll. pygmaea. Fll. longicollis. Mg. caesia. $\mathrm{Mg}$. nalla. Meq. quadrilineata. Mcq. tenuipalpis. Ztt. unicolor. Brullé. pulchella. Lw. flaricincta. Lw. simplicissima. Lw. scalaris. Lw. pilosa. Lw. superciliosa. Lw. 465. M y o pina. R.-Desr. - Musca. Fll. Aricia u. Anthomyza. Ztt. - Coenosia U. Anthomyia. $\mathrm{Mg}$. Cordylura Mg. pt. reflexa. R.-Desv. myopina. Fll. riparia. Fll. pulchriceps. Lw. denticanda. Ztt. longicauda. Ztt. scoparia. Ztt. genarum. Zitt. moriens. Ztt. adscita. Hal. 466. Dialyta. Mg. Nusca. Fll. erinacea. Fll. angulicornis. Ztt. rufifrons. Mg. atriceps. Lw. 467. H om a lom y i a. Bouché. - Mresca. L. F. Fll. Deg. - Anthomyia. Mg. - Aricia. Ztt. - Fannia, Philinta u. Aminta. R. - Desv. - Myantha. Rond. - Coeloniyia. Hal, pt. 
serena. Fll.

pretiosa. Schin.

cmuicularis. L.

domestica minor. Deg.

scalaris. F.

saltatrix. R.-Desr.

manicata. Mg:

armillata. Ztt. ol.

armata. Mg.

macrophthalma. Bouché.

lepida. W.

sociella. Ztt.

incisurata. Zit.

impura. Ztt.

floricola. Mg.

mutica. Ztt.

ornata. Mg.

tlara. Mcq.

subpellucens. Ztt.

canicularis. Ztt.

lugens. Ztt.

tuberculata. Ztt.

perpendicularis. $\mathrm{Ztt}$.

lugubrina. Ztt.

inermella. Ztt.

latifions. Ztt.

spathulata. Ztt.

aprica. Hal.

hamata. Meq.

molissima. Hal.

468. Anthomyia. Mg.

- Musca. L. F. -

Aricia u. Anthomyza.

Ztt. - Anthomyia,

Chortophila, Atomo-

yaster u. Pegomyia. Mcq. - Egeria, Nerina, Adia, Phorbia,

Leveophora, Deliu, Eyle u. Azelia. R.Desv. - Anthomyia, Achantiptera u. Chorsophila. Rond. pluvialis. L.

histrio. Zitt.

plurinotata. Brullé.

albicincta. FIl.

pratincola. Pz.

strigilata. Ztt.

setaria. Mg.

Sunderalli. Ztt. haemorrhoum. Ztt. conformis. Fll. calceolata. Ztt. deprimata. Ztt. flavipalpis. Ztt. mimula. Ztt. maculipes. Ztt. lunatifrons. Ztt. salicis. Ztt. longula. Fll. pullula. Ztt. longula. Mg. buccata. Fll. laminata. Ztt. scatophagina. Ztt. arenosa. Ztt. mistriata. Ztt. albula. Fll. cinerella. Fli. hilarella. Ztt. varicolor. Mg. latipennis. Ztt. albescens. Ztt.

buccata. Fll. pt.

albula. Mg. unilineata. Ztt. parvula. Fll.

sepia. $\mathbf{M g}$. nitidicauda. Ztt. sepiella. Ztt. pusilla. Mg. trapezina. Ztt. transversalis. Ztt. lepturoides. Ztt. pictiventris. Ztt. curvicauda. Ztt. Megerlei. Mg. odontogaster. Ztt. flaviceps. Ztt. geniculata. M[cq.

triquetra. Fll. ambulans, Schrk.

Staegeri. Ztt. triquetra. Ztt. ol. Macquarti. Ztt. triquetra. Meq. aterrima. $\mathrm{Mg}$. tetra. Mg. obscuripennis. Ztt. fuscinervis. Ztt. lanceolata. Ztt. ol. gibbera. Mg. nudipes. Ztt.

Winthemi. Mg. capucina. Ztt. transversa. Fll. invisa. $Z$ tt. consobrina. Ztt. silacea. $\mathbf{M g}$. vittigera. Ztt. ephippium. Ztt. pallido-scutellata. Ztt. tenera. Ztt. gilva. Ztt. flavoscutellata. Ztt. limbatella. Ztt. solenuis. Mg.

Haveola. MIg. inanis. Fll. fulgens. Mg. 
mitis. Mg.

rumicis. R.-Desv.

nigritarsis. Ztt.

hyoscyami. R.-Desv.

bicolor. W.

strigipes. Ztt.

calyptrata. Ztt.

latitarsis. Ztt.

exilis. MIg.

versicolor. Mg:

hyoscyami. Deg. Mg. atriplicis. Gour.

flavipes. Fll.

pulchripes. Lw.

flavipes. Mg.

rufipes. Fll.

viduata. $Z$ tt.

laticornis. Fll.

lactucae. Bouché. pratensis. Mg. antiqua. Mg.

cepparum. Mg.

schisticolor. Ztt.

ruficeps. Mg.

brassicae. Bouché.

fusciceps. Ztt.

spreta. Mg.

cardui. Mg.

brunnescens. Ztt.

gnava. $\mathrm{Mg}$.

platura. Mg.

criniventris. Ztt. (Ari(ia). Ztt.

furcata. Bouché.

fulviceps. Mcq. nigrifrons. Mcq.

basilaris. Mcq.

longipes. R.-Desr.

flarescens. R.-Desr.

myoidea. R.-Desr. thoracia. R.-Desp.

brassicae. R.-Desv.

caepicola. R.-Desv.

radicum. L.

brassicae. W.

napo-brassicae. Bjrk.

vuluaris. R.-Desv.

Friesiana. Bouché.

fugax. $\mathrm{Mg}$.

intersecta. $\mathrm{Mg}$.

brevicornis. Ztt.

floralis. Fll.

angustiventris. Ztt.

obelisca. Míg.

canescens. Ztt.

florilega. Ztt.

octoguttata. Ztt.

promissa. Mg.

striolata. Fll.

discreta. $\mathrm{Mg}$.

dissecta. Mg.

argyrocephala. Mg.

pilifera. Ztt.

piligera. Ztt.

brumnilinea. Ztt.

cinerosa. Zti.

punctiventris. Ztt. (Avicia) Zit.

lineariventris. Ztt. flavifrois. Ztt.

aestiva. $\mathrm{Mg}$.

muscaria. F.

humerella. Ztt.

frontella. Ztt.

aerea. Fll.

vetula. Ztt.

naso. Ztt.

rotundicornis. Ztt. melanura. IIg.

sponsa. Mg.

angustifrons. $\mathrm{Mg}$.

trilinea. $\mathrm{Mg}$.

infirma. $\mathrm{Mg}$.

marginalis. Fll.

depressiventris. Ztt.

ciliatocosta. Ztt.

brunnea. Ztt.

extensicornis. Ztt.

obscura. Meq.

stigmatella. Ztt.

opacula. Ztt.

morionella. Ztt.

albidella. Ztt.

subrittata. Brullé.

socculata. Ztt.

albipennis. Mg.

stigmatica. Mg.

liturata. $\mathrm{Mg}$. agrorum. Mg:

picipes. Mg.

cinerascens. Mg.

diadema. MIg.

rillipes. Ztt.

minuta. Mg.

tabida. Mg.

egens. Mg.

esuriens, MTg.

germana. $\mathbf{M g}$.

triplasia. Mg.

macra. $\mathrm{Mg}$.

memnonipes. Ztt.

frigida. Ztt.

rugulosa. Ztt.

interruptella. Ztt.

atritarsis. Ztt.

dissimilipes. Ztt.

palliceps. Ztt.

scapularis. Ztt.

pubipes. Ztt.

stupida. Ztt. 
Lurida. Ztt.

geniculata. Mcq.

Species incertae.

tibialis. Mg.

amnulipes. Meq.

cana. Mcq.

chorea. R.-Desr.

calthae. R.-Desv.

ludibunda. R.-Desv.

temperata. Mg.

pruinosa. Mg.

polystigma. Mg.

calceata. Mg.

socia. Fll.

inuncta. Ztt.

aeneiventris. Ztt.

nigriventris. Ztt.

argyrata. Ztt.

argyrocephala.Ztt.ol. platyptera. Ztt

maculifrons. Ztt.

lividiventris. Ztt.

squalens. Ztt.

cineren. Fll.

igmobilis. Zt.t.

sulciventris Ztt.

capucina. Ztt. (Aricia. Ztt.)

decrepita. Ztt. vitticollis. Ztt.

dorsata. Ztt.

quinquelineata. Ztt.

umbrisquama. Ztt.

scrupulosa. Ztt.

interruptilinea. Ztt.

gemmata. Ztt.

atricauda. Ztt.

articulata. Ztt.

moerens. Ztt.

cuneicornis. Ztt. anilis. Ztt. murilla. Ztt.

senilis. Ztt.

auctinervis. Ztt.

candens. Ztt.

alulata. Ztt.

trapezoides. Ztt.

barbiventris. Ztt.

lucidiventris. Ztt. rotundiventris. Ztt.

albinervis. Ztt.

lucidula. Ztt.

arrogans. Ztt.

melanogaster. Ztt.

seminitida. Ztt.

conifrons. Ztt.

obtusa. Ztt.

longicornis. Ztt.

glaucescens. Ztt.

lacteipennis. Ztt.

foveolata. Ztt.

veterima. Ztt.

macula. Ztt.

parvicornis. Ztt.

aërea. Fll.

globuliventris. Ztt.

melanaria. Ztt.

nigripennis. Ztt.

cordifrons. Ztt.

mucronatella. Ztt.

parva. R.-Desp.

confidella. Ztt.

dissitinervis. Ztt.

longisquama. Ztt.

alpica. Ztt.

atomaria. Ztt.

sulcella. Ztt.

ignobilis. Ztt.

flavicoxa. Ztt.

limeatipes, Ztt.

apicalis. Ztt.

cineraria. Ztt.

pallipalpis. Ztt. denudata. Ztt.

rerticella. Ztt.

pubiseta. Ztt.

fulviceps. Ztt.

zonata. Ztt.

ruficeps, Ztt. non MIg.

transgressa. Zitt.

guttifrons. Ztt.

tunicata. Ztt.

Holmgreni. Boh.

sordida. Brullé.

semiaenea. Brullé.

quadripunctata. Brullé.

trimacula. Bouché

geniculata. Bouché.

melania, L. Dut.

allecta. TV $1 \mathrm{k}$.

collina. Wlk.

debilis. Wlk.

decedens. Wlk.

defecta. WIK.

defixa. Wik.

depulsa. Wlk.

desulans. Wlk.

detracta. Wlk.

effecta. IVIK.

ellapsa. IVIk.

extrema. WVlk.

fixa. Wlk.

futilis. Wlk.

impulsa. Wlk.

inconfessa. IVlk.

infixa. WVlk.

interlatens. Wlk.

praeterita. WIK.

repulsa. Wlk.

stricta. IVlk.

subsecuta. IVlk.

subtracta. Wlk.

supera. Wlk.

tempta. IVlk.

transiens. WVlk. 
109. H y lem y ia. R.-Dst. - Musca. Fll. Anthomyia. Mg. Aricia U. Anthomyza. Ztt. - Hydrophoria. Neq. pt.

hilaris. Fll. tessellata. Mcq. pallida. Heq. festiva. Ztt. grisea. Fll. virginea. $\mathrm{Mg}$. puella. Mg. flavipemis. Ell. crassirostris. Mg.

variata. Fll. lasciva. Ztt. cinerella. Ng. paralleliventris. Ztt. coarctata. Fll.

leptoyaster. Ztt. ol. fuscula. Fll. strigosa. F.

conica. Fll. vayans. $\mathrm{Pz}$. nigrimana. $\mathrm{Mg}$. sylvicola. R.-Desv. regens. MIg. praepotens. W. linogrisea. Mg. conica. IV. monticola. Ztt. coronata. Ztt. dubitata. Fll. querceti. Bouché. caudata. Ztt. ambiyua. Ztt. ol. linuatocollis. Ztt. ol. munda. Mg. allotalla, $\mathrm{Mg}$. sociata. Mg. partita. Mg. enesia. Meq. (Hylemyia. Mcq.)

rustica. R.-Desv. agrestis. R.-Dest. potamogeti. R.-Desr. hyalipennis. Ztt. tenuiventris. Ztt. verticina. Ztt. frontata. Ztt. distincta. Mg. ruralis. $1 \mathrm{gg}$. villica. $\mathrm{Mg}$. lionesta. Mg. intermedia. $\mathrm{Mg}$. confinis. Mg. xanthopus. Mg. sericata. $\mathrm{Mg}$. amabilis. $\mathrm{Mg}$. mystica. $11 \mathrm{~g}$. conjuncta. Mg. innoxia. $\mathrm{Mg}$. inconspicua. Mg. flavipennis, R.-Dess. sagittariae. R.-Desv. cylindrica. R.-Desv. pallipes. R.-Desv. nigricans. $\mathrm{Mg}$. caesia. Mcq. (Hydrophoria. Meq.)

congenulata. Ztt. trivittata. Ztt. crinirentris. Ztt. (Anthomyza. Ztt.)

lativentris. Ztt. subtessellata. Ztt. immatura. Ztt. rittipes. Ztt. inquirenda. Ztt. lacteipalpis. Ztt. decorata. Ztt. gracilis. Ztt. flavogrisea. Ztt. . vetula. $/ 2 t t$. brunneifrons. Ztt. ferrugineovittata. Ztt. operosa. Mg. aequalis. $\mathrm{Mg}$. strenua. $\mathrm{Mg}$. Lienigii. Gimmerth. Bavescens. Gimmerth. 470. Eriphia. Mg. - Mresca. Fll. Aricia. Ztt. Billbergi. Ztt. aethiops. Ztt.

cinerea. $\mathrm{Mg}$. montana. Schin. sylvestris. Fll. 471. Limophora. R.Desv. - Mrusca. Fll. - Anthomyia. MIg. - Aricia u. Anthomyza.Ztt. - Helina, Limonia, Plullis u. Limnophora. R.-DsF. compuncta. W. didyma. Ztt. trigonifera. Ztt. triangulifera. Ztt. sororcula. Ztt. depressula. Ztt. nupta. Ztt. quadrimaculata. Fll. cornuta. F. litorea. FlI. consimilis . Fll. protuberans. Ztt. comsimilis. Ztt. ol. pertusa. Mg. euphemoides. R,-Dsv. atramentaria. $\mathrm{Mg}$. stygia. Mg. tristis. Mg. 
diaphana. W.

fava. R.-Desr.

gricina. Schin.

varians. Ztt.

leucosticta. Mg.

omissa. Mg.

anicula. Ztt.

brunneisquama. Ztt. contractifrons. Ztt. arctica. Ztt. ol. fumipennis. Ztt. vana. $Z t t$. trianguligera. Ztt. trigonota. Ztt. novemmaculata. Ztt. septemnotata. Ztt. didyma. Ztt. ol. humilis. Ztt. maculipennis. Ztt. surda. Ztt.

borealis. Ztt.

abbreviata. Ztt. cristata. Ztt.

meteorica. Fll. pt. discoidea. Meq fratercula. Ztt. binotata. Ztt. sororia. Ztt. consors. Ztt. obscuripes. Ztt. grandaeva. Ztt. deserta. Ztt. atricolor. Fll. tergina. Ztt. dorsalis. Ztt. niveipennis. Ztt. albibasalis. Ztt. punctiventris. Ztt. maculiventris. Ztt. impar. Ztt. hians. Ztt. mutata. Fll.

fumigata. Mg*.

172. Drymeia. Mg. Musca. Fll.

hamata. Fll. obscura. Mg.

473. Ophyra. R.-Desv. - Musca. Fll. Anthomyia. Mg. Aricia. Ztt.

leucostoma. WT. nitida. R.-Desv. pubescens. R.-Dess. anthrax. Mg. rutilans. R.-Desv. viridescens.- R.-Desy. albipuncta. Ztt. metatarsata. Ztt. spoliata. Ztt.

474. Hydrotaea. R.Desv, - Musca. L. F.Fll. - Anthomyia. Mg. - Aricia. Ztt. - Hydrotaea, Blainvillia und Peronia. R - Desv. - Onodontha. Rond. pt. occulta. MIg. ciliata. F. spinipes. Fll. rostrata. R.-Desv. sylvicola. Lw. dentipes. F. anxia. Ztt. bispinosa. Ztt. armipes. Fll. meteorica. L. vaccarum. Deg. velutina. R.-Desv. brevipennis. Lw. curvipes. Fll. dentimana. Mg. irritans. Fll.

glabricula. Fill. floralis. R.-Desr. bidens. Mg. incompta. Mg. militaris, Mg. blanda. Mg. palaestrica. Mg. nebulosa. R.-Desv. flavifacies. R.-Desv. palpata. R.-Desv. floccosa. Hcq. brunnipennis. Mcq. obscuripennis. Heq. scambus. Ztt. cyrtoneurina. Ztt. 470. Lasiops. Mg. Anthomyia. Mg. ol. - Aricia. Ztt. Thricops. Rond. pt. - Phaonia. R.-Dsr. cunctans. Mg. aculeipes. Ztt. hirsutula. Ztt. apicalis. Mg. simplex. W. postica. Ztt. villana. R.-Desv. depressiventris. $Z t t$. subfuscinervis. Ztt.

vicina. Ztt. orichalcea. Ztt. turpis. Ztt. inconspicua. Ztt. fulvisquama. Ztt. luteisquama. Ztt. pallidisqua ma. Ztt. flavisquama. Ztt. hirtula. Ztt. innocua. Ztt. separ. Ztt. variabilis. Ztt. ol. 
subrostrata. Ztt.

ləucogaster, Ztt. depressiuscula. Ztt. anthracina. Ztt. cinctinervis. Ztt. tristiola. Ztt. pubipes, Ztt. cinereiventris. Ztt. glacialis. Ztt.

hirticeps. Ztt. ol. semipellucida. Ztt. decolor. Fll.

innocons. Ztt.

hirticeps. FIl. eriophthalma. Ztt.

476. Spilogaster Meq. - Musca. Scop. F. Fll. - Anthomyia. Mg. - Aricia und Anthomyza. Ztt. Hylemyia. Mg. pt. - Fellaea, Mydaea, Rohrella u. Mydina. R.-Desr. - Hydrophoria. Meq. u. Rond.

semicinerea. W. pt.

hyalinata. Fll. posticata. $\mathbf{M g}$. quadrum. F.

subpuncta. Fll.

dispar. R.-Dess. anceps. Ztt.

quadrum. Fll.

meditata. Fll. pt.

tigrina. F. pt.

communis. R.-Dest. depuncta. Fll. duplicata. Mg. nigripes, R.-Desv. didyma. Mg. duplaris. Ztt. maculosa. Mg. notata. Fll.

carbonella. Ztt.

nigrinervis. Ztt.

vespertina. Fll.

nigritella. Ztt.

divisa. Mg.

ambigua. Ztt. pt. nigrita. Fll.

uliginosa. Fll.

punctata. R.-Desv.

fuscata. Fll.

trigonalis. Mg.

vittifera. Ztt.

clara. $\mathrm{Mg}$.

demigrans. Ztt。 tetrastigma. $\mathrm{Mg}$.

nigricolor. Fll.

impuncta. Fll.

separata, Mg.

tincta. Ztt.

pagana. $\mathrm{F}$.

scutellaris, R.-Desv.

angelicae. Scop.

deceptoria. Schrk.

urbana. $\mathrm{Mg}$.

fera. R.-Desv.

ambigua. Fll.

longicornis. Bouché.

abdominalis. Bouché.

asella. Mg.

fumosa. $M g$.

denigrata. $\mathrm{Mg}$.

bisignata. Ztt. sordidiventris. Ztt.

ancilla. Mg.

testacea. F.

farillacea. $\mathbf{M g}$.

comta. Mg.

suillorum. R.-Desv.

tibialis. R.-Desr.

maculata. Meq.

fuscipes. Ztt. electa. Ztt.

injusta. Ztt.

flavotibiella. Ztt.

humeralis. Ztt.

detrita. Ztt.

olivacea. Ztt.

graminicola. Ztt.

modesta. Mg.

romiturationis. R.-Dsr. 477. Aricia. R.-Desv.

- Musca. F. Fll. -

Anthomyia. Mg. -

Aricia u. Anthomyza.

Ztt. - Mucrosoma.

Phaonia, Iryllna,

Fellaea, Euphemia.

Trennia u. Rohrella.

R.-Dest.

lucorum. Fll.

laetifick. R.-Desv. dispar. Fll.

obscurata. Mg.

Sahlbergi. Ztt.

lardaria. F'.

marmorata. Ztt.

serva. Mg.

alpicola. Ztt.

ammosa. Ztt.

nivalis. Ztt.

lugubris. Mg.

consobrina. Ztt.

morio. Ztt.

albolineata. Fll.

incana. W.

nemorum. ElI.

nigripes. R.-Dest.

indecisa. IVIk.

plumbea. $\mathrm{Mg}$.

carbo. Schin.

longipes. Ztt.

variabilis. Fll.

umbratica. Mg. 
quadrinotata. Mg. laeta. Fll.

pratensis. R.-Dess. betuleti. Bouché. signata. Mg.

vagatoria. R.-Desy. erratica. Fll.

viarum. R.-Desr.

errans. Mg.

erratica. Fll. pt.

nigricornis. R.-Dest. vagans, Fll.

basalis. Ztt.

perdita. Mg.

exoleta. $\mathrm{Mg}$.

pallida. F.

testacea. Deg.

fragilis. R.-Desv.

rufa. $\mathrm{Mg}$.

populi. Ztt.

variegata. Mg.

scutellaris. Fll.

populi. Mg.

pallida. Fll.

denominata. Ztt.

testacea. L. Duf.

rufiseta. Ztt.

lasiophthalma. Meq.

scutellata. Ztt.

spinicosta. Ztt.

veterana. $Z$ tt.

basalis. Ztt. ol.

laxifrons. Ztt.

atra. Fll.

magnicornis. Ztt. erythrostoma. Ztt. abdominalis. Ztt.

cincta. Ztt.

nigriceps. Ztt.

rufipalpis. Mcq.

punctata. Mcc.

aricioides. $\mathrm{R}$-Dest.

albolineata. Gimmerth.
478. Cinochira. Ztt. atra. Ztt. pinguicula. Ztt.

\section{Muscinae.}

479. Myospila. Rond. - Musca. Auctor. meditabunda. F. 480. Cyrtoneura. Mcq.

- Curtoneura. Mcq.

- Mresca. Auct. Anthomyia. MIg. pt. - Morellia u. Muscina. R.-Desr.

Morellia. R.-Desv.

simplex. Lw.

importuna. Hal.

hortorum. Hal. ol. podagrica. Lw. hortorum. W. agilis. R.-Desr.

Muscina. R.-Dest pascuorum. Mg. stabulans. Fll.

cinerascens. W. pabulorum. Fall. caesia. Mg.

borealis. Ztt. ol. fungivora. R.-Desv. concolor. R.-Desv. nigripalpis. Mcq.

bimaculata. Meq. caerulescens. Mcq. curvipes. Mcq. valga. Ztt. assimilis. Fll. aperta. Meq.
481. Pyrellia. R.-Desr. - Musca. Auct. -

Lucilia. Meq. cadarerina. L. serena. $\mathrm{Mg}$. renea. Ztt. nitida. Mg. polita. Mg. ignita. R.-Desr. cuprea. R.-Desv. calida. R.-Desv. cyanicolor. Ztt.

violacea. Meq. R.Desv.

serena. Ztt. ol. purpureofasciata. Ztt. fasciata. Gimmerth.

482. Dasyphora. R.Dess. - Musca und Cyrtoneura. 1Ig. Lucilia. Mcq. versicolor. Mg. pratorum. $\mathbf{M g}$. cyanella. $\mathrm{Mg}$.

lasiophthalma. Meq. eriophthalma. Mcq. 483. Lucilia. R.-Dest. - Musca. Auct. Phormia.R.-Desr.pt.

Phormia.

regina. $\mathrm{Mg}$. caerulea. Mcq. fulrifacies. Meq. vittata. R.-Desv.

Lucilia. R.-Desv.

nobilis. $\mathrm{Mg}$. caesar. L. ruficeps. Mg. 
latifrons. Schin. sericata. Mg. ? caerulescens. Mcq. cornicina. Fll. caesarion. $\mathrm{Mg}$. sylvarum. Mg: splendida. Mg. illustris. Mig. albiceps. MIg. equestris. Mg. pubescems. R.-Desr. saphirea. R.-Dest. rostrellum. R.-Desv. pallipes. Mg. Haviceps. Meq. fuscipalpis. Meq. fulgida. Ztt. Meigenii. Schin. cornicina. $\mathrm{Mg}$. thalassina. Mg. regalis. Mg. puella. Mlg. parvula. Mg. albipennis. Mg. pruinosa. Mg. calens. R--Dest. fervida. R.-Desy. scutellata. Mcq. chloris. Hal. crerulea. Brullé. violacen. Gimmerth. 484. Musca. L. - Plaxemyia u. Byomyia. IR.-Desv. pt. domestica. L. corrina. $\mathrm{l}$. autumnalis. Deg. ludifica. F. niyripes. Pz. Tau. Schrk, allifions. L. pellucens. Mg. campestris. R.-Desv. aurifacies. R-Desv. bovina. R.-Desr. lateralis. Meq. cuprea. Mleq. riparia. R.-Desv. vitripenuis. $\mathrm{Mg}$. sugilatrix. R.-Desv. camifex. R.-Desv. tempestiva. Fll. nana. $\mathbf{M g}$. phasiaeformis. Mg: pubiceps. Ztt. consobrina. Curtis. 48:. Pollenia. R.-Dsv. - Musca. Auct. Nitellia. R.-Dusv. pt.

Pollenia. R.-Desv.

rudis. $\mathrm{F}$.

obscura. F. vespillo. Mg. cervina. Schrk. amentaria. Scop. mitens. Ztt.

prebescens. R.-Desv. ragrabunda. Mg. obscura. Mg'. fulvicomis, R.-Desv. bicolor. R.-DesF. fulvipalpis. Meq. vernalis. R. $-\mathrm{D}$ usv. vivida. R-Desv. pumila. R.-Desr. floralis. $R$-Desv. ruficeps. Mg. violacea. Meq. rufipalpis. Mg: caerulescens. Meq. recta. Meq. olivacea. Meq.
Nitellia. R.-Desv. atramentaria. Mg. lanio. Pzo varia. Mg. depressa. Mg. nigrina. Mg. ruficornis, Meq. vilesceus. Mcq. ıana. $\boldsymbol{R}_{\mathrm{a}}-\mathrm{Desv}$. intermedia. Mcq. pruinosa. Meq.

macellaria. Hal. degener. Hal. fulvicornis. Ztt. 486. C alliphora. R Desv.-Musca. Auc - Mufetia. R.-Ds pt.

vomitoria. L. carnaria. Scop. mortuorum. I'. fulvibarbis. Meq. carnivora. F'. erytlurocephala. Mg. vomitoria. Hll. Heq. azurea. Fll.

dispar. L. Duf. chrysorhoea. MIg. groenlandica. Ztt. violacea. $\mathrm{Mg}$. soldida. Ztt. affinis. Beq. anthracina. Mg* scutellata. Mcq. micans. Mg.

rufipalpis. Meq. autissiodorensis. R.-D fiavipalpis. $\mathbf{M g}$. 
48\%. Graphomyia. R.Desv. - MLusca. Auct. - Cyrtoneusa. Heq.

maculata. Scop.

vulpina. F.

minor. R.-Dess. picta. Ztt.

caerulescens. Whlbg. 488. M es embrina. Mg. - Musea. Auct. Syrphus. Pz. - Eristalis. F.

meridiana. L.

mystacea. L.

apiaria. F.

bombylius. Deg.

apiformis. $\mathrm{P} z$.

intermedia. $\mathrm{Ztt}$.

resplendens. Whilog.

489. Gymnostyliua.

Meq.

nitida. Hicq.

590. R by n ch omyia. R.-

Desv. - Mlusca.

Germ. - Idia. Lw.

columbina. Mg.

concinna. Germ.

ruficeps. F.

felina. R.-Dest.

speciosa. Lw.

cyanescens. Lw.

fulviceps. Egg.

491. Idia. Mg。 - Musca. F.

fasciata. Mg.

lunata. F.

cinerea. R.-Dest.

492. S tomoxys. Geoffr.

- Musca. Deg. -

Conops. L. - Hacmatobia. Meq. pt. -

Lyperosia. Rond. pt.
Stomoxys, R.-Dest.

calcitrans, L. pungens. Deg.

Haematobia. R.-Desv.

ferox. Meq. pungens. R.-Desp. geniculata.R.-Desr. irritans. L.

pungens, $\mathrm{F}$. serrata. R.-Desv. tibialis. R.-Dest. melanogaster. Mg. stimulans. Mg. irritans. $\mathbf{F}$.

Sarcophaginae.

493. Onesia. R. - Desv. - MLusca. Fll. Mig.

- Sarcophaga. Ztt.

- Lucilia. Mg. pt.

- Melinda. R.-Desv.

sepulcralis Mg: pt.

vespillo. Fll.

riparia. R.-Desv. floralis. R.-Desv. cognata. $M g^{*}$.

caerulea. Mg. ol.

cyanea. Ztt.

gentilis. Mg.

atriceps. Ztt.

genarum. Ztt.

laticornis. Ztt.

agilis. Mg:

pusilla. Mg.

claripennis, R.-Dest.

viarum. R.-Desv.

fulvipennis. Meq.

clausa. Meq.

nigripalpis. Meq. azurea. $\mathrm{Mg}$.

caerulea. Meq.

494. Cynomyia. R.Desv. - Musca. L. F. Ell. - Sarcoph $\alpha-$ ga. Auct.

mortuorum. L. vomitoria. $\mathrm{E}$. chrysocephala. Deg. alpina. Ztt. fuscipalpis. Ztt. 495. Sarcophaga. $\mathrm{Hg}$. - Musca. L. F. Fll. - Myophora, Phorellia u. Agria. R.Dess. - Llaesoxipha. Lw.

Blaesoxipha. Lw.

grylloctena. Lw. haematodes. Mg: haemorhoidalis, Fll. campestris. R.-Desv. hemisphaerica. R.Desv.

erythrocera. R.-Desv. riparia. $\mathrm{R}$-Desv. collinaris. R.-Desv.

Sarcophaga.

atropos. Mig. vagans. Mg. pumila. Mig. striata. F. carnaria. L. vivipara. Deg. varicgata. Scop. colosisus. Scop. cuerulescens. Ztt. albiceps. $1 / \mathrm{g}$. vulnerata. Schin. dalmatina. Schin. 
haemorrhoidalis. $\mathbf{M g}$. cruentata. Mg. ruficauda. Ztt. haemorrhoa. Mg. erythrura. Mg. dissimilis. Mg. offuscata. $\mathrm{Mg}$. nigriventris. Mg. depressifrons. Ztt. grisea. $\mathrm{Mg}$. lineata. Fll. affinis. Fll.

punctata, $\mathrm{R}_{0}$-Desv. melanura. Mg. albiceps. Ztt. sinuata. Mg. intricaria. Mg. arvorum. $\mathrm{Mg}$. clathrata. Mg. laticornis. $\mathrm{Mg}$. humilis. Mg. ruralis. Fll. pygmaea. Ztt. fuliginosa. R.-Desv. agricola. R.-Desv. laticrus. R.-Desv. squamigera. R.-Desv. nana. R.-Desv. arvensis. R.-Desv. florum. R.-Desv. morio. R.-Desv. brunnicans. R.-Desv. albifrons. R.-Desv. Iuteifrons. R.-Desv. campestris, R.-Desv. vicina. Mcq. floralis. Mcq. albida. Mcq. pusilla. Mcq. bipunctata. Mcq. albipennis. Gimmerth.
|496. Sarcophila. Rond.| tessellata. Egg. - Musca. F1l. - 502. Dexia. Mg. Sarcophaga. Mg. - Musea. F. Fll. Agria. Mcq. pt. Meigenii, Schin. ruralis. $\mathrm{Mg}$. magnifica. Schin. latifrons. Fll. maculata. Mg. obsoleta. Fll. tetripunctata. L. Duf. notata. Mcq. pallipalpis. Meq.

497. Theria. R.-Desv. - Sarcophaga. Mg. muscaria. Mg. palparis. R.-Desv.

\section{Doxinae.}

498. Micropthalma. Mcq. europaea. Egg. 499. Phorostoma. R.-

Desv, - Dexia. Mg. Ztt. Egg. - Omalogaster. Meq. - Billaea. R.-Desv. pt. pectinata. $\mathrm{Mg}$.

grisea. R.-Desr. lata. Egg. triangulifera. Ztt. subrotunda. R. - Desv. Schin.

microcera. R.-Desv, maritima. Mcq. 500. S y n t o m o c e r a. Schin. - Dexia. Mg. Egg.

erythraea. Egg. brevicornis. Egg. nigricornis. Egg. 501. Zeuxia. Mg. cinerea. Mg.
Dexilla. Westw. Myocera u. Catilia. R.-Dess. carinifrons. Fll. anthophila. R.-Desv. ferina. Fll.

longipes. R.-Desv. pellucens. Egg. canina. F. vacua. Fll. rustica. F. proletaria. Egg.

irrorata. Mg. marmorata. $\mathrm{Mg}$. atripes. $\mathrm{Mg}$. nigripes. Meq. hirsuta. Meq. nigra. Mcq. nitida. R.-Desv. interrupta. Mcq. testacea. Mcq. dolens. Zt puberula. Ztt. grisea. R.-Desv. curvipes. Ztt. fingens. Wlk. 503. Dinera. Mcq. - Musca. Fll. Dexia. Mg. - Estheria. R.-Desv. pt. - Aria. R.-Desv. grisescens. Fll. cristata. Mg. flavicornis. Mg. grisea. Me q. pygmaea. Mcq. cinerea. Mcq. fulvicrus. R.-Desv. 
804. Prosella. St. Farg.t508. Ny ctia. R. - Dest. et Serv. - Stomoxys. Auct.

siberita. F.

longipes. Gmel.

longirostris. Egg.

505. Mintho. R.-Desv. - Musca. F. Fll. Dexia. Mg. - Ocyptera. F. - Wiedemannia. Mg. - Sericocera. Mcq.

compressa. F.

lateralis. $\mathrm{Pz}$.

praeceps. Scop. rufiventris. Fll. lurida. Schrk. compressa. Mg. hystrix. Enc. méth.

506. Melania. Mg. Musca. F. Fll. Ocyptera. F. - Dexia. Mg. ol. - Sericocera. Mcq. - Phyllomyia und Phorophylla. R.-Desv. volvulus. F. cylindrica. Fll. bifasciata. Mg. palpata. R.-Desv.

507. Thel a ira.R.-Desv. - Musca. Pz. Fll.

- Dexia. Mg. Sericocera. Mcq.

leucozona. $\mathrm{Pz}$. nigrina. Fll. nigripes. F. lateralis. Fll. bifasciata. Mg. - Musca. Pz. F. Dexia. Mg. - $\mathbf{M e}-$ lanophora. Meq. Anthracia. Mg. pt. - Megerlea. R.-Desv.

halterata. $\mathrm{Pz}$. maura. F. carceli. R.-Desv. caminaria. Mg. Servillei. Meq. claripennis. R.-Desv. trifaria. R.-Desp. vernalis. Meq. trifasciata. Meq. 509. Melano phora. Mg. - Musca. L. F. Tachina. Mg. - Ocyptera. Fll. - Tephritis. F.

roralis, L.

grossificationis. L. atra. Meq. appendiculata. Meq. pygmaea. Mcq. rubescens. Mcq. helicivora. Gourreau. 510. Calobataemyia. Mcq. nigra. Meq.

511. Morinia. R.-Dest. - Musca. Fll. Dexia. Mg. - Melanophora. Meq. Melanomyia. Rond. nana. $\mathrm{Mg}$.

parva. R.-Desv. minima. Ztt. melanoptera. Fll. roralis. Schrk. anthracina. $\mathrm{Mg}$. trifasciata. Mcq. sarcophagina. Schin. tergestina. Schin. fimbriata. Mg。 obscura. Mg. pyg maea. Ztt. 512. Medoria. R.-Desv. - Tachina. Mg. Phania. Ztt. pt. luctuosa. Mg. digramma. Mg. melania. Mg. funesta. Mg. glabra. Mg. collaris. $\mathrm{Mg}$. corvina. Mg. acerba. $\mathrm{Mg}$. flavicalyptrata. Meq. pullula. Ztt. incisurata. Ztt. phasiaeformis. Mg.

Tachininae.

513. Phyto. R.-Desv. -

Tachina. Mg. - Rhinophora. Mg. - Ptilocera. Mcq. parvicornis. $\mathrm{Mg}$. melanocephalus. Mg. niger. R.-Desv. obscuripennis. Mg. lepidus. $\mathbf{M g}$. aenescens. Ztt. brunnicornis. Meq. cilipemis. Mcq. rectangularis. Mcq. bicinctus. $\mathrm{Mg}$. tomentosus. Mcq. trilineatus. Mg. celer. Mg. tonsus. Lw. genibarbis. Mg. 
barbatus. Mg. rubicosus. Mg. 31台. Rhinophora. R.- ruficornis. Meq. Desv. - Tachina u.517. L e u c ostom a. Dexia. Mg. - Ocyptera. Fll. - Ptilocera. Meq. - Stevenia. R.-Dsv.pt. - C'assidaemyia. Micq.

femoralis. Mg. simplicissima. Lw. inornata. Lw, atramentaria. Mg. melania. Mg. nitens. R.-Dest. obliqua. Meq. perpendicularis. Meq. umbratica. Fll.

nigripennis. R.-Dsr. lugubris. Ztt. hyalinata. Ztt. tessellata. R.-Desv. nigripennis. R.-Desv. submetallica. Meq. obscuripennis. Lw. subpellucida. Lw. deceptricula. Lw. deceptoria. Lw. nigrans. $\mathrm{Mg}$. fuscipennis. Meq.

515. Plesioneura. Mcq. incisuralis. Meq.

516. Plesina. Mg. Tachina. Mg. - Leucostoma. Ztt. Mcq. Ocyptera. Fll. nigrisquama. Ztt. flavicomis. Ztt. limbata. $\mathbf{M g}$. maculata. Fll. brevicornis. Ztt. liturata. Lw. umbratica. Mg. phalerata. Mg. nubilipennis. Lw. Mg. - Ocyptera. Fll. Taclina. Mg. - Psa. lida. Rond, - Clelia. R.-Desv. pt. simplex. Fll.

rapida. R.-Dest. analis. $\mathrm{Mg}$.

agilis. R.-Desv.

erythrocera. R.-Desv. tetraptera. $\mathrm{M} \mathrm{Ig}$. anthracina. $\mathrm{Mg}$. flavidipennis. Meq. minor. Meq. breripetiolata. Mcq. minor.R.-Dsv. (Clelia). phaeoptera. IIg.

518. Clista. $\mathrm{Mg} .-T_{a-}$ china. MIg. - Microcheilosia. Meq. foeda. Mg. muscina. Schin. moerens. Mg. provida. Mg. diversa. Mg: lentis. MIg. suffiusa. Mg. iners. Mg. lugubrina. Ztt. humeralis. Ztt. heteropalpis. Mcq. maura. Perr.

aberrans. Lw. renosa. $\mathrm{Mg}$. gagatina. $\mathrm{Mg}$.

\section{Microcheilosia.}

nitida. Mer.
319. S copolia.R-Desp. - Mrusca. Pz. Ocyptera. Fll. - Tachina. Mg. - Meianophora. Meq. - Wagneria. R.-Desv. pt. Brachystylum.Meq.pt. morio. Fll. ocypterina. Ztt. carbonaria. Pz. costata. Fll. latifrons. Ztt. cunctans. Mg. lugens. $\mathrm{Mg}$. succincta. $\mathrm{Mg}$. spathulata. Ell. ocypterata. Fll. borealis. Ztt. picta. Ztt. ovata. Meq. gravicornis. Lw. angusticornis. Lw. fulvicornis. Meq. rupestris. R-Desv. rufipes. R-Desv. gagatea. R-Desv. nudioculata. Mcq.

In rachystylum. Mcq. nigra. Meq. 520. Microsoma. Meq. nigra. Hcq. 521. Ageulocera. Neq. nigra. Meq. 52. Hypostena. Mg. - Clausicella. Rond. pt. - Tachina. Ng. procera. Mg.

cylindracea. Ztt. medorina. Schin. incisuralis. Meq. setiventris. Mcq. humeralis, Perr. maritima. Perr. 
couvexinervis. Meq. suturata. Rond.

523. Hyperecteina. Schin.

metopina. Schin.

524. Degeeria. Mg. Tachina. Auct. Metopia. Meq. Medina. R.-Desv. Elophorina. R-Desv. collaris. Fll.

cylindrica. R-Dest. cylindroidea. Mcq.

ornata. Nig. separata. Mg. blanda. Fll. seria. Mg. selecta. Mg. parallela. Mg. tragica. Mg. amica. $\mathrm{Mg}$. grandicornis, Ztt. albomaculata. Mco. fuscanipennis. Meq. caerulescens. Mcq. pygmaea. Mícq. minima. Mcq. fascinans. $\mathrm{Mg}$. muscaria. Fll. infuscata. Fll. strigata. Mg. turrita. Mg. pulchella. MIg: ambulatoria. $\mathrm{Mg}$. hystrix. Ztt. convexifrons. Ztt. semicincta. Ztt. abbreviata. Ztt. maculisquana. Ztt. decorata. Ztt. albicincta. Ztt. fasciata. Meq. incisuralis. Meq. flavisquamis. R.-Desv. Carcelli. R.-Desv. albifrontalis. Gimm. 525. Macquartia. R.Dess. - Tachina. Auct. - Nemoraca. und Erebia. Meq. Zophomyia. Mcq. Erebia, Amedea und Albinia. R-Desv. pt. atripes. $\mathrm{Mg}$.

lucida. Mg. diaphana. Fll. dispar. Fll. rufipes. Meq. rubripes. Meq. tibialis. Mcq. brachycera. R.-Desv. flavipes. MIg. monticola. Egg. chalconota. Mg: major. Schin. temebricosa. Mg. affinis. Schin. grisea. Fll. dispar. Fll. pt. eyens. Mg. atrata. Fll. corrusea. $\mathrm{Mg}$. nitida. Ztt. atrata. $\mathrm{Mg}$. tristis. $\mathrm{Mg}$. flavicalyptrata. Meq. chalybeata. Mg. nigrita. Fll. prolixa. Mg.

spinosa. Ztt. griseicollis. Mg. tricincta. Mg. Corimna. Mg. praefica. $\mathrm{Mg}$. trimaculata. Mg. haematodes, Mg. nigripennis. $\mathrm{Mg}$. ochropus. $\mathbf{M g}$. callida. Mg. germanica, R,-Desv. microcera, R.-Desy. scutellaris. R,-Desv. buccalis. R.-Desv. albinervis. Ztt. angulicornis. Zitt. ? grisea. Ztt. rotundicornis. Ztt. umbrosa. Ztt. fulvicornis. Ztt. crucigera. Ztt. ciliaris. $Z$ tt. rufipalpis. Meq. varipalpis. Mcq. albicalyptrata. Meq. flavipenmis. Mfcq. nudistylum. Mcq. perpendicularis. Mcg. clausa. MIcq. caelebs. Heq.

gymnophthalma. Mcq. nudioculata. Mcq. 326. Loewia. Egg. Tachina. Ztt. Macquartia. Rond. setibarba. Egg. puncticeps. Ztt. brevifrons. Rond. occlusa. Rond.

527. Petaguia. Rond. subpetiolata. Rond. 3̈28. Friraldsky i a. Schin. - Tachina. HIl. - Fallenia. Mg. 
longicornis. Fll. coracina. Mg.

529. Polidea. Mcq. Tachina. Auct. Macquartia. R.-Desv. - Zophomyia. Meq. Harrisia. Mg. nenea. Mg. vividescens. R.-Desv. simplicitarsis. Ztt. rebaptizata. Rond. conspersa. Mg. crassitarsis. Ztt. idiotica. Mg. pagana. Mg. 530. Tryphera. Mg. Tachina. MIg. succincta. Mg. umbrinervis. Ztt. fulvipes, Mg. lugubris. $\mathrm{Mg}$. flavida. Mg. tesellum. $\mathrm{Mg}$. interruptella. Ztt. amplicornis. Ztt. grossicornis, Ztt. Fischeri. Gimmerth. nigrifacies. Perr.

531. Clytia. R.-Desv. Musca. F. Pz. - Tachina. Mg. Fll. Eliozeta. Rond. pt. rotundiventris. Fll. continua. $\mathrm{Pz}$. tephra. Mg. helvola. Mg. hellvo. F. pellucens. Fll, dalmatica. R.-Desr. cylindrica. R.-Desv. vaga. R.-Desr. macrocera. Heq. auriceps. Micq. fumipennis. Ztt. punctiventris. Ztt. 532. Melia. R.-Desv. Tachina. Mg. - Actia. Mg. - Myobia u. Thryptocera. Meq. albipennis. R.-Desr. leucoptera. Mg. zonaria. Lw.

533. Phy tom yptera. Rond. nitidiventris. Rond. aberrans. Schin.

534. Siphona. Mg. Afusca. Deg. - Stomoxys. F. Fll. - Bucentes. Ltr,

cristata. F.

tachinaria. Mg. geniculata. Deg. minuta. F. cinerea. $\mathrm{F}$. Meigenii. Enc. méth. nigrovittata. Enc. $m$. pusilla. R.-Desv. quadrinotata. R.-Dsr. consimilis. R.-Dest. fuscicornis. R.-Desv, tristis. R.-Desv. analis. Enc. méth. flavifrous. Staeg. maculipennis. Mg. maculata. Ztt. anomala. Ztt. palpina. Ztt. clausa. R,-Desv. sylratica. R.-Desv. testacea. R.-Desv.

humeralis. R.-Desv. melanocera. R.-Desv.
535. Thryp tocera. Med. - Tachina. Mg. Fll. - Herbstia, Actia, Osmaea, Neaera und Ramburia. R.-Desv. - Bigonichaeta und Neaera. Rond.pt. Elfia, Vafrellia. u. Ceranthia. R.-Desv.

Neaera. R.-Desv.

laticornis. Mg: immaculata.R.-Desv. albicollis. MIg. atra. R.-Desv.

Bigonichaeta. Rond. setipennis. Fll. Mariettii. Rond.

Thryptocera. Heq.

frontalis. Mcq. pilipennis. R.-Desv. lamia. $\mathrm{Mg}$. obscurella. R.-Desv. cingulata. R.-Desv. vitripennis. Rond. silacea. $\mathrm{Mg}$. crassicornis, Mg. pilipennis. Fll. pt. pilipennis. Fll. flavisquamis. R.-Desv. latifrons. $\mathbf{M g}$. coguata. Schin. fracticornis. Mg. exoleta. Mg. tibialis. Rond. magica. $\mathrm{Mg}$. curvicornis. Mg. zonata. Mg. spinipennis. $\mathbf{M g}$. delecta. Mg. 
nigrina. Mg. Fersicolor. Fll. infantula. Ztt. zonella. Ztt. minutissima. Ztt. rufina. Ztt. ciliata. Mcq. llavipalpis. Meq. antennalis. Rond. nigripalpis. R.-Dest. humeralis. R.-Desr. grisea. R.-Dess. (Ceromyia.)

spathulata. R-Desr. fulvipes. R.-Desv. tibialis. R.-Desv. bicolor. Mg". microcera. R.-Desr. testacea. R.-Desr. rubifrons. R.-Desv. erythrocera. R.-Desv. grisea. R.-Desr. $\mathrm{CO}_{\mathrm{s}-}$ maea.)

ludibunda. R.-Desv. vivida. $R$.-Dest. 536. Roeselia. R.-Desv.

- Tashina. Fll. Mg.

- Myobia. Mg. ol.

- Eurigaster. Mcq. antiqua. Fll.

arvensis. R.-Desv. agrestis. R.-Desy. cylindrica. R.-Desr. sylvatica. R,-Desv. interrupta. R.-Desr. llarisquamis. R.-Desv. flavescens, R.-Desv. 333. Myobia. R.-Dest.

- Tachina. Mg. Fll. - Solieria u. Orillia. R.-Desv. pt. melaleuca. Mg. fenestrata. Mg. longipes. $\mathbf{M g}$. inanis. Fll. fragilis, R.-Desv. cinerascens. R.-Dest. ruficrus. R.-Desv. vicina. R,-Dess. modesta. R.-Desv. pacifica. Mg. aequa. Mg. hospes. $\mathbf{M g}$. femorata. Meq. lateralis. $\mathbf{M g}$. nitens. R.-Desv. nana. R.-Desv. flavida. Meq. longirostris. Meq. dubia. Meq. pumila. Neq. lestremensis. Mcq. nitidiventris. Mcq. frontalis. Meq. micans. Meq. fulvipalpis. R-Desv. sublutea. R.-Desr. raga. R.-Desv. testacea. R.-Desv. villana. R.-Desv. festiva. R.-Desv. binotata. R.-Desv. gagatea. R.-Dest. immaculata. R.-Desv. rustica. R.-Desf. elongata. R.-Desv. fuscana. R.-Dess. femoralis. R.-Desv. brunnicosa. R,-Desr. dimidiata. $R$-Desv. germana. R,-Dest. cincrea. R.-Desv. nigra. R.-Dest. rectinervis. R.-Desp. curvinervis. R,-Desv. pellucida. R,-Desv. fuscipalpis. Perr. latipennis. Perr. varipes. Perr. 538. Leskia. R. - Desv. - Tachina. Mg. Myobia. Mg. - Pyrosia. Rond. - $\mathrm{Fi}$ scheria. R.-Desv. pt. aurea. Fll.

flatesens. R.-1)est.

bicolor. R.-Dest.

539. Redtenbacheria. Schin. - Tachina. Mg.

phaniaeformis. Egg. insignis. Egg. biguttata. $\mathbf{M g}$. 540. Braueria. Schin. - Zelleria. Egg. longimana. Egg. 54. Haliday a. Egg, = argentea, Egg. aurea. Egg.

542. Phylloteles. Lw. pictipenuis. Lw.

533. A podacra. Hcq. Tachina. Ztt. seriemaculata. Meq. pulchra. Egg. elegantula. Ztt. 54. Miltog ram ma. Mg。 - Tachina. Fll. Sphixapata.Roud. pt.

Sphixapata. Rond. intricata. Mg. conica. Fll. maculosa. Rond. melanura. Mg:. 
grisea. $\mathbf{M g}$.

albifrons. Rond.

lineolata. Rond.

pygmaea. Rond.

Pelopei. Rond.

Piccioli. Rond.

imberbis. Ztt.

fasciolella. Ztt.

convexula. Ztt.

minuta. Fll.

puncticoruis. Ztt.

interrupta. Mcq.

Aliltogramma. Rond.

ruficornis. $\mathrm{Mg}$.

vutilans. Mg.

melitensis. Mcq.

murina. Mg.

pilitarsis. Rond.

oestrrcea. Fll.

tessellata. $\mathrm{Mg}$.

Germari. $\mathbf{M g}$.

punctata. Mg.

pilimana. Rond.

Contarinii. Rond.

fasciata. Mg.

taeniata. Mg.

arcuata. Ztt.

rotundiventris. Ztt.

griseola. Fll.

oculata. Ztt.

deprimata. Ztt.

tachinaria. Ztt.

immaculata. Mícq.

V-nigrum. Mleq.

nigricormis. Mcq.

amifrons. L. Duf.

parasita. R.-Desr.

Megerlei. Mg. incompta. $\mathrm{Mg}$. globularis. $\mathrm{Mg}$. sexpunctata. Mg.

tricuspis. $\mathrm{Mg}$.

strennua. Perr.

ruficornis. Gimmerth. brevipennis. Bigot.

5.5. Hilarella. Rond.

- Miltogramma. Ztt.

- Megaera. R.-Dsv.

siphonina. Ztt.

dira R.-Desv.

Zetterstedtii. Rond.

hilarella. Ztt.

italica. Roud.

plumicornis. Ztt.

atrox. R-Desv.

fera. R.-Desv.

inimica. R.-Desv.

incurra. Mcq.

nitida. Mcq.

nigra. Meq.

angustifrons. Meq.

546. He te ropterin a.

Meq. - Miltogramma.

Mg. - Taxigramma.

Meq. ol.

stictica. Mg.

heteroneura. $\mathbf{M g}$.

multipunctata. Rond.

pusilla. Ztt.

pipiens. Perr.

547. M с г 0 n c h i a.

Rond. - Tachina.

Fll. - Miltogramma.

Mg. -- Oodiyaster.

Mcq. pt. - Amobia.

R.-Desv.

agrestis. Fll.

conica. R.-Dest.

polyodon. Mg.

anomala. Ztt.

cylindrica. Fll.

cincta. $\mathrm{Mg}$.

signata. Mg.
548. Tinnertzia. Schin.

- Metopia. Lw.

mesomelaena. Lw.

549. Paragusia. Schin.

Frivaldskyi. Schin.

550. Metopia. Mg. Musca. F. Pz. - Tachina. Fll. - Araba u. Ophilia. R.-Desr. Steinii. Sclin.

fastuosa. Mg. leucocephala. Rossi.

labiata. F.

argyrocephala.Ztt.ol. campestris. Fll. argentata. Mcq. argyrocephala. Mg. amabilis. $\mathbf{M g}$. biseriata. Mcq. sinuata. Meq. rubricornis. MLcq. nasuta. Mg. perfida. Mg: tincta. $\mathrm{Mg}$. convexinervis. Meq. rubrifrons. Mcq. forficulae. Newport. cinerea. Perr. crassicornis. Perr. 5้อ1. Frontina. Mg. Tachina. Ztt. - Metopia u. Phorocera. Mcq. - Masicera. Mg.pt. - Latveillia. R.-Desv. pt. - Fabricia. $\mathrm{MIg} \cdot \mathrm{pt}$.

laeta. $\mathrm{Mg}$.

testacea. R.-Dsv.

laetabilis. Ztt. nigricans. Egg. tibialis. Meq. 
aprica. MIg. austera. Mg.

demissa. Mg。

Fabricia. $\mathrm{Ig}$.

pacta. Mg.

รั.2. Baum ha ueria. Mg.

- Musca. F. - Tachina. Fll. - Eurigaster. Mcq. pt. Phryxe. R.-Dest.pt. goniaeformis. $\mathrm{Mg}$.

grandis. Egg.

vertiginosa. Fll.

marmorata. F.

larvicola. R.-Desv. gracilis. "Egg.

albocingulata. Fll. scutellaris. Fll.

533. Phorocera. R.Desr. - Tachina. Mg. - Blondelia, Pales, Medina, Rhinomyia, Latreillia u. Phorocera. R.-Desr. - Metopia, Lydella. Meq. - Pericheta, Chetogenu, Leucanipa, Machaerea, Botria, Campylocheta u. Istocheta. Rond. Doria. Mig. pt. Chetogen a. Rond. caesifrous. Meq. assimilis. Rond. assimilis. Fll.

agilis. R.-Desv. grandis. Rond. filipalpis. Rond. segregata. Rond. gramma. Mg. acuminata. Roud. media. Rond. patellifera. Rond. Doria. IIg.

concinmata. $\mathrm{Mg}$. prorsae. R.-Dest.

Guerini. R.-Desv. pieridis. R.-Desv. serriventris. Rond. meditabunda. Mg. stupida. Mg. distincta. $\mathbf{N g}$. bellatrix. Ztt. trizonata. Ztt. nigripalpis. Rond. mobilis. Ztt. caeruleonigra. Perr. nigra. Perr.

Phorocera. R.-Desv.

obscura. Fll.

scutellata. R.-Desv. frontosa. Mg. cilipeda. Rond. pumicata. Heq. pumicata. $\mathrm{Mg}$. fiorea. R.-Desr.

Blondeli. R.-Desv. delecta. Mg. rufipalpis. Mg. pantherina. Ztt. elongata. Mcq. pullata. Mg. tessellata. Meq. minuta. Meq. raripalpis. Meq. leucomelas. Mg. angusta. Mcq. familiaris. Mg. albiceps. Mg. inepta. Mg. munda. Mg. obliquata. Mg.

lata. Zutt.

rufiscutellatris, $Z$ tt.

mundula. Ztt.

dalecarlica. Ztt.

squamosa. Ztt.

picipes. Rond.

polleniella. Rond.

grisella. Rond.

verecunda. Rolid.

strenua. R.-Desy.

aurulenta. Perr.

lata. Perr.

scutellata. Perr.

petrosa. R.-Dest.

viridescens. R-Desr.

vernalis. R.-Desv.

laevigata. R.-Uess.

ortalidis. R.-Desv.

orgyae. R.-Desv.

cuculliae. R.-Desv.

Bercei. R.-Desr.

pusilla. R.-Desr.

taeniata. Mg.

cinerea. Neq.

parida. Mg.

praecox. $\mathbf{M g}$.

aestuans. Mg.

unicolor. Fll.

frontosa. Rond. (Istocheta.)

schistacea. Mg.

Lamberti. R.-Desv. pascuorum. Rond. pudibunda. Fisch.

354. Eggeria. Schin.-

Tachina. Ztt. -

Spoggosia. Rond. -

Fallenia. Eg:日.

fasciata. Egg. ribrissata. Ztt. 
pectinata, Ztt.

clausa. Ztt.

occlusa. Rond.

๒55. Gaedia. Mg. -

Tachina. Mg. ol.

connexa. Mg.

distincta. Schin.

๖̋6. Masicera. Meq.

Tachina. Fll. Mg. -

Phryxe, Carcelia. Ly-

della. R.-1)esr. -

Blepharipa, Cero-

masia. Rond. pt.

proxima. Egg.

cgens. Egg.

rutila. Mg.

scutellata. R.-Desr.

obliqua. R.-Desv.

ciliata. Meף.

ferruginea. Mg:

micans. IIcq.

florum. Heq.

brevis. Meq.

lutescens. Meq.

fatua. Mg.

analis. Meq.

maculifacies. Mcq.

montium. Heq.

unicolor. Mícq.

nitida. Mcq.

multisetosa. Mcq.

Aarifrons. Meq.

rirescens. Mlcq.

buccata. Mg:

fuscipennis. Meq.

media. Mieq.

tessellata. Meq.

myodaea. R.-Desr.

angusta. Meq.

interipta. Mcq.

syiratica. Fll.

superba. R.-Desr.

tiphaecola. Mcq. major. Meq.

ruficornis. Meq.

cespitum. Mcq.

pulverulenta. Mcq.

distincta. R.-Desr.

socia. Meq.

cylindrica. Meq.

Bremii. Mleq.

flaridipennis. Meq.

atropirora. R.-Desv.

Roberti. Mcq.

acutangulata. Meq.

montana. Meq.

declivicornis. Meq.

pachystyla. Heq.

badensis. Meq.

hannoniensis. Meq.

pinetorum. M $\mathrm{ICq}$.

nitens. Meq.

innoxia. IIg.

consobrina. Mreq.

palustris. Meq.

aenescens. Heq.

flavescens. Mcq.

albocincta. Meq.

latipennis. Heq.

parrula. Meq.

parra. Meq.

cinerea. Mcq. non Fll. flavoscutellatí. Ztt.

pratensis. Mg.

cinerea. Fll.

pumila. Mg.

a urulenta. Mg".

bicinta. Mg:

melaleuca. $\mathrm{Mg}$.

rufitarsis. $\mathbf{M g}$.

senilis. Mg.

geniculata. $/ \mathrm{tt}$.

furibunda. Ztt.

usta. Ztt.

scutellata. Ztt. ralidicornis. Zte.

grisea. R.-Desy.

Blondeli. R.-Desv.

hemisphaerica. R.-

Desv.

luteipalpis. R.-Desr

ranessae. R.-Desv.

floricola. R.-Desr.

concolor. R.-Desy.

tibialis. R.-Desv.

evocata. Wlk.

intersecta. IVIk.

laticincta. Perr.

nova. Perr.

atropicida. Perr.

palpalis. Perr.

grisea. Perr.

minor. Perr.

cylindrica. Perr.

clausa. Perr.

exigua. Perx.

rubrifrons. Perr.

35\%. Tachina. Meig. - Musca. L. E. Oodigaster. Mcq. pt. - Voria u. Acemyia. R.-Desr. - Brachy. coma. Rond, pt.

polita. Mg.

mimula. Mg.

viduata. $\mathrm{Mg}$. marginella. Mg.

vidua. $\mathrm{Mg}$.

larrarum. L.

praepotens. Mg.

fasciato. Hll.

litoralis. R.-Desy.

flavescens. Mg:

scutellaris. R-Desv.

mescens. R.-Desr.

villica, R,-Desr.

macrocera. R.-Dest. 
erucarum. Rond. presilla. Meq. celer. Meq. Havicalyptrata. Meq. angustifasciata. Mcq. angusta. Meq.

rustica. Mg.

larvarum. Mg. simulans. Mg. ludibunda. Meq. rectinervis. Mcq. fiavifrors. Mcq. pumila. Mcq. albifrons. Mec. alacer. MLeq.

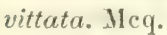
flavipalpis. Mec audens. Meq. angelicae. $\mathrm{Mg}$. acuticornis. $\mathrm{Mg}$. vagabunda. $\mathrm{Mg}$. grisescens. Mg. doris. Mg. bibens. Mg. agilis. IIg. morosa. $\mathrm{Ng}$. erratica. $11 \mathrm{~g}$. tibialis. Mg. aestiva. Mg. aestivalis. Meq. nigripes. Fll. bella. Mg: opaca. Mg. subfisciata. Mg. retusta. Mg. hortensis. $\mathrm{Mg}$. gratiosa. Mg. dorsalis. $\mathrm{Mg}$. parasita. Mg. inumbrata. Mg. taeniota. Mlg. breripennis. Mg.
Amasia. Mlg. pagana. $\mathrm{Mg}$. inconspicua. Mg. urbana. Mg. albisquama. Ztt. apicalis. Meq. auriceps. Mg. brevicornis. Heq. spectabilis. $\mathrm{Mg}$. lepida. Mg. fallax. Mg. illustris. Mg. aurifrons. Mg: stimulans. MIg. otlusca. Mg. nitidula. $\mathbf{M g}$. speculatrix. Mg. vallata. $\mathrm{Mg}$. stabulans. Mg. viatica. $11 \mathrm{~g}$. terminalis. Mg. incompta. Mg". funebris. Mg. incana. Fll. sybarita. Mg. occulta. $\mathrm{Mg}$. subrotunda. Heq. oblouga. Mcq. virginea, $\mathbf{H g}$. agnita. Mg. quadrinota. $\mathrm{Mg}$. flaviceps. Mcq. nitidiventris. Heq. velox. Mcq. rufifrolls. $\mathbf{M e q}$. rivida. Meq. levicula. Nicq. albiceps. Mcq. arcuata. Meq. caeruleifrons. Mcop. testaceo-lateralis. $\mathbf{v e r}$. bilineata. Neq. gracilistylum. Meq. inflexicoruis. Meq. distantipenuis. Meq. micans. Meq. caesifrons. Mcq. flaridipennis. Mcq. rapida. R.-Desv. fasciata. Mcq. tenax. Mcq. rubriscutellata. Meq. pallidipalpis. Mcq. macroglossae. $R$-Desr. nitidiventris. Ztt. samnio. $\mathrm{Ztt}$. vilis. $Z$ tt. futilis. Ztt. sordidisquama. Ztt. pictiventris. Ztt.

maculiventris. Boh. ludio. $\mathrm{Ztt}$. vicina. $Z$ tt. incurva. Ztt. scutelligera. Ztt. spathulaecornis. Ztt.

spathulaefornis. Ztt. nigrisquama. Ztt. circumflexa. Ztt. tachinoides. Fll. pubicornis. Ztt. cingulata. Ztt. macularia. W. festiva. R.-Desv. chrysalidarum. Rond. brucorum. Rond. civilis. Rond. glossatorum. Rond. nympharum. Rond. metopiellą. Rond. devia. Fll. policheta. Egg. nigricans. Egg. demotica. Egg. 
ignota. Perr.

nbscurella. Ztt.

rubritarsis. Ztt.

maculiventris. Ztt.

semizonata. Ztt.

halterata. Ztt.

petiolinervis. Ztt.

villicornis. Ztt.

nitens. Ztt.

cyrtoneurina. Ztt.

flaritarsella. Ztt.

pusilla. Ztt.

Lindemanni. Gimm.

Gimmerthali. Fischer.

accidens. Whk.

augens. Wlk.

caminaria. Wlk.

certans. Wlk.

collecta. WIk.

neglecta. Wlk.

commissa. Wlk.

comitata. Wlk.

comosa. WVlk.

computa. WIk.

conducta. Whk.

confecta. Wlk.

defecta. Wlk. 1.

conjuncta. Wlk.

constans. Wlk.

contempta. Wlk.

defecta. Wlk. 2.

delitescens. Wlk.

demissa. Wlk.

demota. Wlk.

denotans. Wlk.

discrepans. Wlk.

disjuncta. WIk.

dispecta. Wlk.

dispuncta. Wlk.

distenta. Wlk.

distermina. Wlk.

dirulsa. Wlk. domator. Wlk.

effecta. Wlk.

emissa. Wlk.

enodata. Wlk.

enotata. Wlk.

erecta. Wlk.

erogata. Wlk.

evidens. Wlk.

eroluta. Wlk.

exacta. Wlk.

exagens. Wlk.

excessa. Wlk.

exclusa. Wlk.

interclusa. Wlk. 1.

expedita. Wlk.

expleta. Wlk.

exscensa. Wlk.

exsecta. Wlk.

intersecta. WVlk. 1.

fissa. Wlk.

Hexa. Wlk.

immissa. Wlk.

infensans. Wlk.

infestans. Wlk.

infixa. Wlk.

inoperta. Wlk.

inquilina. Wlk.

insedata. Wlk.

insuscepta. Wlk.

intacta. Wlk.

intaminata. Wlk.

intercedens. Wlk.

intercepta. Wlk.

interclusa. Wlk. 2.

interlapsa. Wlk.

interlatens. WIk.

intermixta. Wlk.

interna. Wlk.

internexa. Wlk.

intersita. Wlk.

intersecta. Wlk. 2.

inroluta. Wlk.

mera. Wlk. motor. Wik.

multans. Wlk.

munita. WIk.

nana. Wlk.

nexa. Wlk.

nigrolineata. Wlk.

objecta. TVlk.

particeps. Wlk.

perpingens. Wlk.

pertinens. Wlk.

pertracta. Wlk.

quadricincta. Wlk.

reclusa. Wlk.

refecta. Wlk.

reformata. Wlk.

rejecta. Wlk.

retracta. Wlk.

reventa. WIk.

senta. Wlk.

separata. Wlk.

viridulans. Wik.

processionea. Rtzb.

iliaca. Rtzb.

ochracea. Rtzb.

similans. Rtzb.

janitrix. Rtzb.

pinivora. Rtzb.

larvincola. Rtzb.

monacha. Rtzb.

neustriae. Rtzb.

Hartigii. Rtzb. evonymella. Rtzb. gilba. Hrtg.

inclusa. Hrtg.

bimaculata. Hrtg.

piniariae. Hrtg.

erythrostoma. Hitg.

inflexa. Bouché.

558. M e igenia.R.-Dest.

- Tachina. Fll. Mg.

- Masicera. Mg. pt.

- Spylosia. Rond. 
majuscula. Rond. bisignata. $\mathrm{Mg}$. discolor. Ztt. aprica. Ztt. borealis. R. - Desv. cylindrica. R.-Dest. immaculata. R.-Dsv. favescens. R.-Dest. grisescens. R.-Dest. quadrimaculata. Meq. dumetorum. Mcq. limaculata. Mg. floralis. Fll.

minuta. Mreq. vernalis. R.-Desv. nitida. R.-Desv. bicolor. MIcq. mutabilis. Fll.

? floralis. Mg. errans. $\mathrm{Mg}$.

559. Exorista. Mg.

Tachina Fll. - Senometopia, Lydella u. Eurigaster. Mreq. Hubneria, Carcellia, Dorbinia, Phryxe, Phryno u. Zenillia. R. - Desv. - Winthemia. R.-Desv. pt. Lomachanta, Aporomyia. Rond. - Lypha. R.-Desv. vulgaris. Fll. distans. Meq. audax. Meq. scutellaris. Mcq. florida. Meq. magnicornis. Ztt. crinita. Rond. cincinna. Rond. capillata. Rond. confinis. Fll. ronata. R.-Desv. agnata. Rond. ancilla. $\mathbf{M g}$. barbatula. Rond. nemestrina. $\mathrm{MIg}$. tritaeniata. Rond. dubia. Fll. sylvatica. R.-Desv. agrestis. R.-Desv. nitida. R.-Desr. berberidis. $\mathrm{Mg}$. fimbriata. Mg. commixta. Ztt. arrensis. Mgg. nemea. $\mathbf{M g}$. vetula. MIg. fulvipes. Rond. basalis. Mg. canescens. Meq. longicornis. Perr. biserialis. Mcq. arguta. R.-Desv. comata. Rond. excisa. Fll.

flavicans. Meq.

rutila. Rond. excavata. Ztt. festinans. Mg. diluta. Mg. rufoscutellata. Mcq. properans. Rond. lucorum. $\mathrm{Mg}$. crassiseta. Rond. separata. Rond. gnava. Mg. bombylans. R.-Desv. proxima. Mg.

fulva. Fll. cheloniae. Rond. leucophaea. MIg. nigripes. R.-Desv. falenaria. Rond. polycheta. Meq. libatrix. $P_{Z}$. fauna. Mg.

Macquarti. R.-Desr. lota. Mg.

ruficauda. Ztt. apicalis. Mg. saltuum. Mg. alacris. MIg. affinis. Fll. arvicola. Mg. hortulana. Mg. jucunda. Mg. diadema. Mg. angustipennis. $\mathrm{Mg}$. glauca. Mg. heraclei. Mg. mitis. MIg. festiva. Mg.

Bohemani. Ztt. albicans. Fll. perturbans. Ztt. dolosa. Mg. ferina. Mg. prominens. Mg. floralis. Mg.

Westermanni. Ztt. flavicalyptrata. Mcq. sanguinolenta. Meq. vinulae, R.-Desv.

rasa. Micq. meditata. Mg. nobilis. R.-Desy. platycera. Mcq. pertinax. Mreq. aemula. $\mathbf{M g}$. laticella. Mcq. pabulina. Mg. arrogans. MIeq. sepium. Meq. notata. MIeq. pusilla. Meq. cauta. Mcq. puparum. R.-Desr. vernalis. R,-Desv. 
rufiventris, Meq. gibbicornis. Mcq. major. Meq. appendiculata. Meq. uticola. Mcq. rapida. $\mathbf{M g}$. rivida. Meq. minuta. Mcq. inclinata. Meq. consanguinea. Mcq. ardens. Mcq. brevis. Mcq. levis. Mcq. dumetorum. Mleq. imparida. Mcq. insinuans. Meq. terminalis. Meq. singularis. Meq. caerulescens. Meq. concava. Meq. griseifrons. Meq. agoilis. R.-Desv. flovida. R,-Desr. athalia. R.-Dess. punctata. R.-Dess. Havifrons. Mcq. fraterna. Mcq. nitida. Mícq. pallipes. Fll. agilis, R.-Desv. brunnea. R.-Desf. auvulenta. R.-Desv. angelicae. Mcq. levicola. Mcq. acronyctarum. Mcq. modesta. $\mathrm{Mg}$. brunnicornis. Meq. elliptica. Mcq. aenescens. Mcq. curiensis. Mcq. parvula. Meq. aeris. Meq. longicornis. Mcq crassistylum. M[eq. cita. Meq. diffusa. Meq. habilis, Mcq. rolatica. Mcq. ambulans. Mg. aratoria. $\mathrm{Mg}$. nectarea. $\mathbf{M g}$. popularis. $\mathbf{M g}$. puella. $M \mathrm{~g}$. lepida. R.-Desv. rernalis. R.-Desr. orgyae. R.-Dest. acronita. R.-Desv. cuculliae. R-Desv. eruceti. R,-Desv. Bercei. R.-Dest. Arion. R.-Desv. amoena. R.-Desv. bombycivora. R-Desr. aurifrons. R.-Desv. nigripes. R-Desr. relox. $R$-Desv. rustica. R.-Desr. flavipalpis. R.-Desv. parra. Meq. grandis. Ztt. paroniae. Ztt. maculipennis. Ztt. demens. Ztt. obesa. Ztt. stulta. Ztt. porcula. Ztt. rufotibialis. Ztt. ferrugineotibialis. Ztt. linearicornis. Ztt. Bonstorffi. Ztt. cornuta. Ztt. spernenda. Ztt. diligens. Ztt. fulvifrons. Ztt. praetervisa. Ztt. bicingulata. Ztt. bizonata. Ztt. genarum. Ztt. ornaticornis. Ztt. rotundaticornis. Ztt. straminifrons. Ztt. duplinervis, $Z t t$. villica. $Z$ tt. subpruinosa. Ztt. aretica. Ztt. hyalinata, Ztt. fractiseta. Rond. noctuicida. Rond. fugax. Rond. sussurans. Rond. glixina. Rond. confundens, Rond. caudata. Rond. acanthina. Rond. cognata. Rond. parens. Rond. aberrans. Rond. aristella. Rond. parra. Rond. temera. $M g$. pabulina. $\mathbf{M g}$. 560. Epicampocera. Mcq. - Táchina.Mg. - Evorista. Mg. Heq.

succincta. MIg. curvicornis. Mcq.

561. Nemoraea. R.Desv. - Musca. F. - Tuchina. Fll. Mg. - Emestia, Fausta, Mericia, Erigone, Panzeria, Meriania, Pherbellia $u$. Winthemia. R.-Desr. Platychira, Chetolyga, Chetina, Nemorilla. Rond. 
glabrata. MIg. pellucida. Mg. neglecta. Mg. bombylans. R.-Desv. affinis, R.-Desr. fulva. R.-Desr. conjuncta. Rond. rubica. $\mathrm{Mg}$. nupta. Rond. puparum. F. tricincta. FIl. sylvatica. R.-Desr. borealis. R.-Desv. microcera. Meq. breviseta. Ztt. argentifera. $\mathrm{Mg}$. argentigera. Ztt. latifrons. Rond. strenua. Mg. vagauls. $\mathrm{Mg}$. picea. $\mathrm{Mg}$. vivida. $Z$ tt.

lurida. Fll. pt. setosa. Mcq. tessellans. Egg. consobrina. Rond. rudis. Fll.

lateralis. R.-Desv. consobrina. $\mathrm{Mg}$. tessellans. Meq. radicum. E.

anthophila. R.-Desv. lurida. R.-Desv. scutellaris. R.-Desv. dubia. R.-Desv. minor. Heq. tessellans. R.-Desv. viridulans. R-Desv. cresia. Fll.

Cetolyga. Rond. pt. xanthogastra. Rond. speciosa. Egg. amoena. Mg.

analis. Meq. nigrithorax. Egg. quadripustulata. F. aestuans, Fll.

cinerea. R.-Desv. erythrura. Mg. variegata. Mg. catocalae. R.-Desv. cruentata. Rond. cilicrura. Rond. cilitibia. Rond. pilifera. Rond. separata. Rond. anrulenta. R.-Desv. crassicornis. R.-Desv. flarescens. R.-Desv. nigripalpis. R.-Desv. viarum. R.-Desv.

Nemorilla. Rond.

maculosa. $\mathrm{Mg}$. floralis. Fll. pt. notabilis. Mg. floralis. Rond. aristalis. Rond. amica. Rond. pumila. Rond. soror. Rond.

Thrycolyga. Rond.

minor. Rond. major. Rond. nova. Rond.

Chetina. Rond.

palpalis. Rond.

nemorum. Mg. laevigata. $\mathrm{Mlg}$. renusta. Mg.

protuberans. Zit. recta. $\mathrm{Mg}$.

varia. Mg. ragabunda. Mg. floricola. Mg. appendiculata. Mcq. soror. Meq. nigroscutellata. MIeq. varicornis. Meq. lateralis. Mcq. acutangularis. Mcq. unicolor. Meq. Bremii. Mcq. ignobilis. $\mathrm{Mg}$. histrio. Mg. flaripennis. R.-Desr. erigonea. R.-Desv. floren. R.-Desv. nigra. R.-Dest. scutellaris. R.-Desv. abdominalis. R.-Desv. viridescens. R,-Desv. nerrosa. R.-Desv. brunnicornis. R.-Desv. obliqua. R.-Dest. scutellata. R.-Desv. rubricornis. Ztt. connivens. Ztt. vulnerata. Ztt. conjugata. Ztt. congenita. Ztt. piliceps. Ztt. strigiceps. Ztt. vulneraticornis. Ztt. intermedia. Ztt. fumata. Ztt. hyalipennis. Ztt. tricingulata. Ztt. strigifrons. Ztt. truncata. $Z$ tt. corylana. Gimmerth. xylosteana. Gimmerth. 
neglecta. Wlk.

contracta. Wlk.

dispartita. Wlk.

detracta. Wlk.

bijuncta. WVlk.

intracta. Wlk.

miyophoroidea.R.-Desv. 562. Trixa. $\mathrm{Hg}$. - Cra-

meria. R.-Desv.

alpina. Mg.

caerulescens. Mg.

oestroidea. R.-Desr.

Imhoffi. Ncq.

Amsteini. Mcq.

dorsalis. Mg.

grisea. $\mathrm{Mg}$.

ferruginea. $\mathrm{Mg}$.

variegata. Mg.

limbata. Ztt.

lapponica. Ztt.

obscura. Ztt.

scutellata. Newm.

563. Cnephalia. Rond.

- Tachina. Mg.

bucephala. Mg.

fuscisquama. Rond.

albisquama. Rond.

multisetosa. Rond.

ö64. Go nia. Mg. - Musca. Deg. - Tachina.

Fll. - Reaumuria,

Isomera, Rhedia u.

Spallanzania. R.Desv.

GOnia.

atra. Mg:

bicincta. Mg.

Foersteri. Mg.

fasciata. $\mathrm{Mg}$.

Desvoidyi. R.-Desr.

ruficeps. Wlk.

dirisa. $\mathbf{M g}$. trifaria. Zllr. capitata. Mg.

vittata. $\mathrm{Mg}$.

bombylans. Mcq.

vacua. Mg.

capitata. Deg.

vernalis. R.-Desr.

fulva. R.-Desv.

testacea. R.-Desv.

diversa. R.-Desv.

ornata. Mg.

lateralis. Zllr.

maculipennis. Egg.

nerrosa. Mg.

simplex. Zlir.

puncticornis. Mg.

auriceps. $\mathbf{M g}$.

lusitanica. MIg.

nudifacies. Mcq.

vicina. R.-Desv.

melanura. R.-Dest.

Blondeli. R.-Desv.

parisiaca. R.-Desv.

fasciventris. Mcq.

riridescens. Guér.

maritima. Perris.

Spallanzania.

hebes. Fll.

gallica. R.-Dest.

tessellata. Mcq.

picea. R. - Desv.

Haviceps. Ztt.

cilipeda. Rond.

cinerascens. Rond.

cognata. Rond.

565. Germaria. R.-Dest.

- Tachina. Fll. -

Gonia. Mg. Zt.t. -

Illigera. Mg. - Pis-

semyia. R.-Desv.

ruficeps. Fll.

latifrons. R.-Dest. angustata. Ztt.

goniaeoides. Ztt.

566. P a c h y st y l u u.

Meq.

Bremii. Mcq.

rugosum. Mik.

arcuatum. Mik.

5067. Peteina. Mg. -

Musca. F. - Ta-

clina. Fll. - Seri-

cocera. Mcq.

erinacea. F.

5̋68. Plagia. Mg. -

Tachina. Fll. Mg. -

Cyrtophlooba, Blepha-

rigena. Rond. pt.

ruricola. $\mathrm{Mg}$.

nigripalpis. Rond.

ruricola. Ztt.

elata. Mg:

marginata. Mg.

cuneicornis. Ztt.

trepida. $\boldsymbol{M g}$.

? subcincta. Ztt.

subcincta. Ztt.

curvinervis. Ztt.

ambigua. Mg.

ruficornis, Ztt.

ruralis. Fll.

verticalis. $\mathbf{M g}$.

transversa. Mcq.

ambigua. Fll.

interrupta. Ztt.

369. La bidigaster.

Meq. - Tachina. Mg.

- Dionaea u. Clelia.

R.-Desr. - Cassi-

demiyia. Meq. pt.

forcipata. Mg.

aurulans. R.-Desr.

agilis. R.-Desv.

intermedia. Mcq. 
370. Zophomyia. Mcq.

- Musca. Scop. F.

- Tachina. MIg. -

Macquartia.R.-Desr. pt. - Minella. R.- $3 \%$. Rhamphina. Mcq.

Desv. - Erebia. Mg.

- Avernia. Rond.

temula. Scop.

tremula. F.

nitida. R.-Desr.

nitens. Mcq.

vicina. Meq.

flavipalpis. Mcq.

571. Olirieria. R.-Desr.

- Musca. F. Deg.

- Ocyptera. F. Fll.

- Tachina. Mg. -

Sericocera. Mcq. -

Panzeria. Mg.

argyreata. $\mathbf{M g}$.

lateralis. $\mathbf{F}$.

rufomaculata. Deg.

tachinaria. Fll.

sanguinea. $\mathrm{Mg}$.

572. Demoticus. Mcr.

- Tachina. Fll. Mg.

- Myobia, Solieria.

R.-Desr. pt.

plebejus. Fll.

soror. Ztt.

frontatus. Boh.

cognatus. Egg.

spretus. Mg.

pruinosus. $\mathrm{Mg}$.

arcuatus. Mcq.

pulverulentus. R.-Dest.

573. Aphria. R. - Desv.

- Rlamphina und

Rhynchosia. Meq. -

Olivieria. Mg. - Ceromyia. R-Dest. pt.

- Thryptocera. Mcq. pt. longirostris. Mg.

abdominalis. R.-Dest.

Serrillei. R.-Desr.

suavissima. Lw.

- Stomoxys. Mg. Allertia. Rond.

pedemontana. $\mathbf{M g}$.

575. G y m n o che ta.

R.-Desv, - Tachina.

Fll. Mg. - Chrysosoma. Meq.

aurata. Fll.

viridis. Fll.

rufipes. $\mathrm{Mcq}$.

576. Schineria. Rond.

tergestiua. Rond.

577. M icropalpu s. Meq.

- Tachina. Fll. Mg.

- Linnemyia, Bone-

tia,Bonellia.R.-Desy.

rulpinus. Fll.

sylvestris, R.-Dest.

fulgens. Mg.

heraclei. R.-Desr.

analis. R.-Desf.

borealis. R.-Desv.

aestivalis. R--Desv.

comptus. Rond.

haemorrhoidalis. Fll.

pudicus. Rond.

impudicus. Rond.

nubilus. MIg.

rapidus. $\mathbf{M g}$.

analis. Meq.

comptus. Fll.

marmoratus. Mg.

pictus. $\mathrm{Mg}$.

intricatus. Mg.

Sophia. R.-Desr. oenanthi. R.-Desr.

longipes. R,-Desv. tessellans. R,-Desv.

rubiginosus. Meq.

lateralis. R.-Dest.

vicinus. Meq.

consobrinus. Meq.

longirostris. Meq.

lithosiophagus. Rond.

larvarum. Passerini.

fiater. Rond.

578. Cuphocera. Mreq.

- Micropalpus. Mg.

pt. - Palpibraca u.

Cyphocera. Rond.

ruficormis. Meq.

haemorrhoidata. Rond.

pyrogaster. Rond.

579. Echinomyia. Dumér. - Musca. L. F. - Taclina. Fll. Mg. - Servillia, Fabricia, Taurella und Peleteria. R.-Desr.

Fabricia. R.-Dest.

ferox. $M g$.

Echinomyia.

tessellata. F.

nigricornis. Mg. R.-

Desr.

ferina. Ztt.

grossa. L.

Marklini. Ztt.

regalis. Rond.

fera. $L$.

virgo. $\mathrm{Mg}$.

vubricornis. Mcq.

intermedia. R.-Desv.

errans. R.-Desr.

vernalis. R.-Desv.

testacea. R,-Desr. 
ruficornis. Mg.

fulviceps. Mg.

magnicornis. Ztt.

praeceps. Mg*

pusilla. Mcq.

prompta. Mg.

rubecens. R-Dest.

argentifrons. Meq.

argentifera. $\mathbf{M g}$.

Lefebrrei. R.-Desv.

punctata. R.-Desv.

tessellata. Meq. non F.

sphiricera. Mcq.

fuscanipennis. Mcq.

ruficeps. Mcq.

Prolilli. Costa.

alpina. Ztt.

aenea. Ztt.

tetramera. Ztt.

casta. Rond.

meridionalis. R.-Desv. pedemontana. Meq. abdominalis. R.-Desv.

Servillia. R.-Dest.

Iurida. F.

leucocoma. $\mathrm{Mg}$.

cuculliae. R-Desv.

lateralis. R.-Desv. ursina. Mg.

pilosa. R.-Desv.

echinata. Mg.

subpilosa. R.-Desv.

\section{Planinae.}

̈380. Uromyia. Mg. Tachina. Fll. - Phania. $\mathrm{Mg}$. ol. - Weberia. R.-Desv. pt. curvicauda. Fll.

appendiculata. R.-

Desp. flaripalpis. Meq.

thoracica. $\mathbf{M g}$.

381. Gymnopeza. Ztt. denudata. Ztt.

albipennis. Ztt.

582. M i c r a. Ztt.

trixina. Ztt.

383. Besseria. R--Dest.

- Tachina. Mg. -

Ocyptera. Fill. -

Gymnosoma. Mg. pt.

- Wahlbergia. Ztt.

melanura. Mg.

lateralis. Fll.

reflexa. R,-Desv.

dimidiata. Ztt.

584. Phania. IIg.

vittata. $M g$.

obscuripennis, $\mathbf{M g}$.

lateritia. $\mathrm{Mg}$.

\section{Ocypterinae.}

585. Clairvillia. R.Desv.

ocypterina. Schin. pusilla. R.-Desr.

586. O cyptera. Ltr. Musca. L. F. - Syrphus. Pz. - Besseria. Parthenia u. Ocyptera. R. - Desv. Ocypterula u. Exogaster. Rond. pt. rufipes. MIg. morio. Brullé. nigrita. $\mathrm{Mg}$. rufifrons. Lw. rubida. Lw, bicolor. Enc. méth. coccinea. $\mathrm{Mg}$. pentritomae. R.-Desv. interrupta. Mg. cylindrice. Fll. setulosa. Lw. excisa. Lw. pilipes. Lw. xylotima. Egg. brevicornis. Lw. brassicaria. F. cylindrica. Deg. segnis. $\mathrm{Pz}$. auriceps. $\mathbf{M g}$. intermedia. Mg. cylindrica. F. scalaris. Lw. coaretata. Lw. dimidiata. Enc. méth. cassidae. L. Duf. carimata. I,w. crassa. Lw scapularis. Lw. Boscii. Mcq.

pusilla. Mg. gracilis. Lw.

fascipennis. Lw. 587. Lophosia. Mg. fasciata. Mg.

Gumnosominae. 588. Cistogaster. Ltr. - Syrphus. $\mathrm{E}$-. Tachina. F. Mg. Gymnosoma. Fll. Mg. - Pallasia. R.-Desv. globosus, k? dispar. Fll. ovatus. Mcq. aurantiacus, Mg. anicula. MIg. dimidiatus. $\mathbf{M g}$. obsoletus. Mg. 
589. Gymnosoma. Mg. Musca. L. F. - Tachina. Mg. F. - Ocyptera. Ltr. rotundata. L. costata. Pz. nitens. $\mathrm{Mg}$.

\section{Phasinae.}

590. Syntomogaster. Schin. Phasia. Ztt. pt. - Tryphera. Mg. pt. - ? Campogaster. Rond. - ? Strongygaster. Meq.

viduus. Egg.

singularis. Egg.

ruficeps. $Z t t$.

opacus. Ztt.

convexus. Ztt.

globulus, $\mathrm{Hg}$.

delicata. Mg.

exiguus. $\mathrm{Mg}$.

591. Xysta. Mg. - Thereva. F. - Pliasia. MIg. ol.

holosericea. F. cana. $\mathrm{Mg}$. semicana. Egg. grandis. Egg.

cilipes. Mg. striginerris. Ztt. ? grisea. Ztt. 592. Ananta. Mrg. Elomyia. R. - Desr. - Phasia. Mg. ol. nebulosa. $\mathrm{Pz}$. lateralis. Mg. niyra. Mleq. claripennis. R.-Desv. albovillosa. Mcq. alliseta. Mcq. ol. aurulans. Micq.

flaviventris. Meq.

abdominalis. Meq.

ornata. Mg.

punctata. Mg.

593. Phasia. Ltr.

Syrphus. F, Pz.Mlusea. Pz. - Thereva. $\mathrm{F}$.

analis. F.

dimidiata. Pz.

ancora. Mg.

axillaris. $\mathrm{Mg}$.

basalis. Mg.

brachyptera. Pz.

taeniuta. Pz.

diluta. $\mathrm{Mg}$.

flaviventris. Mg.

dispar. Rond.

dissimilis. Rond.

crassipennis. F.

discoidea. Mg.

varia. $\mathrm{Mg}$.

arvensis. R.-Desv.

vagans. Mg.

rostrata. Egg.

oblonga. Mcq.

nigra. Mcq.

discoidalis. Meq.

Rothii. Ztt.

ว94. A lo ph or a.R.-Desv.

- Conops. L. -

Syrphus. F. Pz. -

Thereva. F. - Phasia. 11 g. - Hyalomyia. R.-Dest. pt. hemiptera. F. subcoleoptrata. F. affinis. F.

obscuripennis. Mg. hemiptera. $\mathbf{M} \mathbf{c q}$. aurigera. Ëgg.

subcoleoptrata. L.

aurulans. Mg.

hamata. Mg.

muscaria. Fll.

cinerea. $\mathrm{F}$.

obesa. F.

speciosa. Curtis. umbrata. Ztt.

nubilipennis. $\mathrm{Mg}$.

bucephala. Mg.

umbripennis. Mg.

violacea. Mg.

atropurpurea. $\mathbf{M g}$.

nebulosa. $\mathrm{Mg}$.

nuteculosa. $\mathrm{Mg}$.

nev'osa. Mg.

hyalipennis. Fil.

semicinerea. Mg.

pusilla. $\mathrm{Mg}$.

grisea. Ztt.

albipennis, Mg.

nana. FU.

pygmaea. Fll.

fuscipennis. Meq.

nebulosa. R.-Desr.

basalis. R.-Desv.

carbonaria. R. - Desv.

Corimma. R,-Desv.

fasciata. Meq.

flavipennis. Ztt.

zonella. Ztt.

fasciola. Ztt.

dispar. L. Duf.

vitripenuis. Ztt.

XXVI, 0 e stridae.

595. Gastrophilus.

Leach. - Oestrus.

Auctor. - Gastrus.

Mig. 
equi. $\mathrm{F}$.

bovi. L.

intestinalis. Deg.

haemorrhoidalis. Gml.

bengalensis. Meq. antilopes. Pallas.

inermis. Brauer.

ferruginatus. Brauer ol.

pecorum. F.

vituli. F.

jubarum. $\mathrm{Mg}$.

Selysii. Cart.

ferruginatus. Ztt.

flavipes. Oliv.

lativentris. Brauer.

haemorrhoidalis. L.

nigricornis. Lw.

nasalis. L.

veterinus. Clk.

salutiferus. Clk.

Clarkii. Leach.

salutaris. Mg.

jumentorum. IIg.

duodenalis. Schwb. nigritus. Ztt.

596. Hypoderma. Ltr.

- Oestrus. Auct. -

Oedemagena. Ltr. pt.

Hypoderma.

Silenus. Brauer.

Diana. Brauer.

Actaeon. Brauer.

? elaphi. Schrk.

lineatus. Kellner.

lineata. Vill.

haemorrhoidalis. Clk.

pt.

ericetorum. Leach.

vernalis. Clk.

bovis. $\mathrm{Mg}$. pt. bovis. Deg. subcutaneus. Greve. bovinus. Schwab.

Bellieri. Bigot.

Oedemagena.

tarandi. L.

rangiferinus. L.

597. 0 e s t r o m y i a.

Brauer. - Hypo-

derma. Braver ol. -

Oestrus. Pallas.

Satyrus. Brauer.

598. Oestrus. L. - Cephalomyia. Ltr. oris. L.

sinus fiontis. L. ovinus. Schwab.

purpureus. Brauer. 599. Cep ha lom y ia. Ltr.

- Oestrus. Schin.pt. maculata. IV.

titillator. Clk.

libycus. Joly.

600. Pharyngom yia.

Schin. - Oestrus.

Auct. - Cepheno-

myia. Brauer ol.

picta. Mg:

cervi. Clk.

601. Cephenomyia. I.tr. - Oestrus. Auct. rufibarbis. Mg.

auribarbis. Mg.

trompe. Kelluer.

Ulrichii. Brauer.

trompe. Modeer.

nasalis. L. pt.

tarandi. Clk.

stimulator.

microcephalus. Clk.

trompe. Clk.

biangulatus. Cooke.

\section{b. Polyneura.}

XXYII. Platypezidae.

602. Callomyia. Mg. Dolichopus. F. Heteroneura. Ell. amoena. Mg. Fallenii. Mg. Meyerlei. Mg. leptiformis. Fll.

Baumhaueri. Mg. antenuata. Ztt. cinerea. Ztt.

Hoffmannseggii. $\mathbf{M g}$. elegans. Mg.

speciosa. Mg.

Fabricii. Schin.

elegans. F.

boreella. Ztt.

Dahlbomi. Ztt.

elegantula. Ztt.

dives. Ztt.

elegantula. Fll.

scutellaris. Ztt.

viduella. $Z$ tt.

Zetterstedtii. Ztt.

603. Platy peza. Mg. -

Dolichopus. F.

holosericea. $\mathbf{M g}$.

boletina. Fll.

fasciata. F.

subfasciata. Mg.

atra. Fll.

aterrima. Wlk.

dorsalis. Mg.

infumata. Hal.

vittata. Ztt.

ornata. Mg.

furcata. Fll.

rufa. $\mathrm{Mg}$.

rufiventris. Meq. 
consobrina. Ztt. media. Mcq. modesta. Ztt. picta. $\mathrm{Mg}$.

fasciata. Fll.

fumipennis. Ztt.

fumipennis. WVlk.

connexa. Boh.

604. Platycuema. Ztt.

- Empis. Fll. -

Cyrtoma. Ztt. ol. Atelestus. WIk.

pulicaria. Fll.

sylvicola. Wik.

tibiella. Ztt.

605. Opetia. Mg.

nigra. Mg.

lonchopteroides. Curt.

\section{XXVHI. Pi puneulidac.}

606. Cha larus. TVlk. -

Pipunculus. Mg. Ztt.

- Cephalops. Fll. -

Ateleneura. Mcq.

spurius. Fll.

velutinus. Mcq.

obscurus. Ztt.

holosericeus. $\mathrm{Mg}$. exiguns. Hal.

607. Pipunculus. Ltr.

- Microcera. Mg. ol.

- Cephalops. Fll.

furcatus. Schin.

auctus. Fll.

modestus. Hal.

sylvaticus. Mg.

nigritulus. Ztt.

elegans. Schin.

campestris. Ltr.

spinipes. Mg.

cephalotes. Bosc.

dentipes. Mg. rufipes. $\mathbf{M g}$.

ruralis. $\mathrm{Mg}$.

pratorum. Fll.

ater. Mg.

flavipes. $\mathrm{Mg}$.

fuscipes. Ztt.

fusculus. Zitt.

geniculatus. $\mathbf{1 l g}$.

unicolor. Ztt.

varipes. $\mathrm{MIg}$.

zonatus. Ztt.

halteratus. $\mathrm{Mg}$.

albitarsis. Ztt.

fascipes. Ztt.

fulvipes. Mcq.

haemorrhoidalis. Ztt.

maculatus. Wlk.

lateralis. Meq.

obtusinerris. Ztt.

opacus. Fll.

pilosus. Ztt.

rittipes. Ztt.

annulipes. Ztt. pt.

608. Nephrocerus. Ztt.

- Pipunculus. Mg. II cq.

flavicornis. Ztt. seutellatus. $\mathbf{H c q}$. lapponicus. Ztt.

\section{Syrphidae.}

Syrphinae.

609. Bacha. F. - Syiphus. F, ol. - Musca. Gmel.

elongata. F.

tabida. $\mathbf{M g}$.

sphegina. Mg.

vitripennis. $\mathrm{Hg}$.

abbreviata. Mg.

scutellata. Mg.

nigricornis. Schumm nigripennis. Mg. obscuripennis. Mg.

Klugii. Mg.

610. Sphegina. Mg. Syrphus. Pz. - Milesia. Fll.

clunipes. Fll. podagricus. Pz. nigra. Mg.

Loewii. Zllr. elegans. Schumm. Hava. Meq. nigricornis. Meq. Zetterstedtii. Schin. nigricornis. Ztt.

611. Ascia. Mg. - Syr. phus. F. Pz. - M Yilesia. Pz. Fll. - Merodon. $\mathrm{F}$.

lanceolata. $\mathrm{Mg}$. podagrica. F. dispar. Mg. floralis. $\mathrm{Mg}$.

bifasciata. Ztt.

dispar. Ztt. quadripunctata. Mg: interrupta. Mg. nitidula. Mg. aenea. Mg. hastata. MIg. geniculata. Mg. maculata. Meq.

612. Doros. $\mathrm{Mg}$. - Syrphus. Pz. Mg. ol. Milesia. F.pt. - Eristalis. F. pt. - Seasva. Fll, - Bucha.

Enc. méth. conopseus. F. coarctatus. Pz. 
613. Tanthogramma. Schin. - Musca. Deg. - Syrphus. F. Mg.

- Evistalis. Ztt. -

Scaeva. Fll. - Doros.613. P elecocera. Mg. Mg. pt.

citrofasciata. Deg.

festiva. Fll. Mg.

ornata. $\mathrm{MIg}$.

festiva. Fll. pt.

dives. Rond.

marginalis. Lw.

614. Melithreptus. Lw.

- Musca. L. - Syrphus. Mg. - Scaeva.

F. Fll. - Sphaero-

phoria. St. Earg. u.

Serv. - Conops. Scop. strigatus. Staeg.

scriptus. L.

gemmatus. Scop.

dispar. Jw.

nigricoxa. Ztt.

pictus. Mg.

incisus. Lw.

menthastri. L.

formosus. $\mathrm{Egg}^{\circ}$.

Loewii. Ztt.

nitidicollis. Staeg.

taeniatus. II'lk.

melissae. Ztt. $\mathrm{p}^{\mathrm{t}}$.

taeniatus. $\mathbf{M g}$.

hieroglyphicus. Mg.

melissae. $\mathbf{M g}$.

philanthus. Mg.

larandulae. Meq.

analis. Mcq.

sinuatus. Meq.

origani. Meq.

limbatus. Heq.

multipunctatus. Ztt.

flavicauda. Ztt.

abbreviatus. Ztt. dubius. Staeg.

serpylli. Rond.

oleandri. Rond.

insignitus. Ztt.

-. Brachyopa. MIg.

pt. - Rlingia. Ell. pt.

scaevoides. Fll.

tricincta. $\mathrm{Mg}$.

flavicornis. $\mathrm{Mg}$.

latifrons. $\mathrm{Lw}$.

flavicomis. Schin. ol. lugubris. Perris.

616. Didea. Meq. - Syrphus. Mg. - Scaeva. Fll. - Enica. Mg.

alneti. Fll.

glaucius. $\mathrm{Pz}$.

pellucidus. $\mathrm{Mg}$.

fasciata. Meq.

Foersteri. Mg.

intermedia. Lw.

617. Syrplius. F. Musca. L. - Scaeva.

Fll. Ztt. - Lasiophticus. Rond. pt.

pyrastri. L.

transfugus. F.

Gemellarii. Rond.

seleniticus. Mg.

laternarius. Miller.

glaucius. Mg.

mutatus. Ztt.

glaucius. L.

nobilis. Mg.

leucozonius. Ahrens.

tarsatus. Ztt.

hilaris. Ztt.

venustus. $\mathrm{Mg}$.

berberidis. Lw.

macularis. Ztt.

implicatus. $\mathbf{H g}$. solitarius. Ztt.
Lunulatus. $\mathbf{~}$ fg.

tricinctus, Fll.

Eggeri. Schin.

albostriatus. Fll.

confusus. Egg.

laetus. F.

novus. Rond.

topiarius, Mg.

ribesii. WIk.

ammulipes. Z $\mathrm{tt}$.

obscurus. Ztt.

Bellardii. Rond.

coronatus. Rond.

dignotus. Rond.

leiophthalmus. Schin. Eg.g.

arcuatus. Fll.

lapponicus. Ztt.

corollae. F.

pyrorum. Sclirk.

olitoria. Fll.

lacerus. Mg.

luniger, $\mathbf{M g}$.

Braueri. Egg:

guttatus. Fil.

nitidulus. Ztt.

lasiophthalmus. Ztt.

grossulariae. Mg.

ribesii. Fll. pt.

diaphanus. Ztt.

ochrostoma. Ztt.

nitidicollis. $\mathbf{M g}$.

ribesii. L.

vitripemis. Mg.

confinis. Ztt.

melanostoma. Ztt.

excisus. Ztt.

affinis. Lw.

abbreviatus. Ztt.

modestus. MIg.

auricollis. Mg.

crenatus. Mcq. 
nigrifemoratus. Meq. flaviventris. Mcq. fulrifrons. Meq. macilentus. $\mathrm{Mg}$. latifasciatus. Mreq. nigritarsis. Ztt. relictus. Ztt. sexmaculatus. Ztt. unifasciatus. Ztt. flaviceps. Rond. bucculatus. Rond. curvipes. Boh. nigritibius. Rond. annulatus. Ztt. umbellatarum. F. maculicornis. Ztt. triangulifer. Ztt. decorus. $\mathbf{M g}$. baltcatus. Deg. alternatus. Schrk. nectareus. $\mathrm{Pz}$. canabinus. Scop. bifasciatus. F. interruptus. Gmel. lineola. Ztt. vittiger. Ztt. cinctellus. Ztt. cinctus. Fil. placidus. $\mathrm{Mg}$. sexguttatus. Mg. sexnotatus. Mg: albifrons. v. Ros. pusillus. v. Ros. bimaculatus. F. Ros. concinnus. Mg. cinctipes. Ztt.

hyperboreus. Staeg:

618. M ela nos toma. Schin. - Musca. L. - Syrphus. F. $\mathbf{M g}$. - Scaeva. Fill. Ztt. hyalinata. FH. dubia. Ztt. barbifrons. Fll. cingulata. Egg. ambigua. Fll. mellina. L. mellaria. $\mathrm{Mg}$. melliturga. Mg. scalaris. F.

Iris. Mg. gracilis. Mg. stictica. Mg. laevigata. Mg. pumicata. Mg. unicolor. Meq. octomaculata. $\mathrm{v}$. Ros. transfuga. Ztt. maculosa. Mg. minuta. Mcq. concolor. Wlk.

619. Platy cheirus. St.

Farg. u. Serv.

Scaeva. F. Fll.

Syrphus. Mg. F. ciliger. Lw. fasciculatus. Lw. melanopsis. Lw. manicatus. Mg. alpicola. Schumm. parmatus. Rond. albimanus. F. cyaners. Miller. peltatus. Mg. scutatus. Mg. podagratus. Ztt. fulviventris. Mcq. ferrugineus. Micq. clypeatus. $\mathrm{Mg}$. immarginatus. Ztt. ferrugineus. Ztt. podagratus. Ztt. pt. quadratus. Meq. rostratus. Ztt. latimanus. Whlbg.

dilatatus. Meq.

scambus. Staeg.

clypeatus. Ztt. pt.

spatulatus. Rond.

620.Py o ph a e n a.Schin.

-.- Syrphus u. Scaeva.

Auct. - Cheilosia.

$\mathrm{Pz}$. - Platycheirus. Staeg.

ocymi. F.

lobatus. Mg.

granditarsus. Foerst. Irlk.

rosarum. T.

notilucus. Pz.

621. S patiga ster. Rond.

- Spazigaster. Rond.

- Milesia. F. - Syrphus, Mg. Lw. F.

ambulans. $\mathrm{F}$.

dispar. Lw.

Apenini. Rond.

lugubris. Rond.

692. Chleilosia, Mg. Musca. L. - Syrplus. F. Fll. Mg. Eristalis. Ztt. - Milesia. F.

oestracea. L. rupestris. Pz. intonsa. Lw. fraterna. Ztt. pigra. Lw. barbata. Lw. vulnerata. Pz. frontalis. Lw. decidua. Eggg. variabilis. $\mathrm{Pz}$.

ater. $\mathrm{F}$.

nigrita. $\mathrm{E}$. nigrina. $\mathrm{Mg}$. 
rostrata. Ztt.

coracina, Ztt.

melanopa. Ztt.

personata. Lw.

derasa. Lw.

gagatea. Lw.

venosa. Lw.

laeviventris. Lw.

rhyuchops. Egg.

sparsa. Lw.

antiqua. $\mathrm{Mg}$.

Schmidtii. Ztt.

nigripes. Mg.

vicina. Ztt.

cocmeteriorum. Ztt.

tropica. $\mathrm{Mg}$.

anthraciformis. Mg.

pubera. Ztt.

maculata. Fll.

insignis. Lw.

crassiseta. Lw.

caerulescens. Mg.

soror. Ztt.

fulvicornis. Lw.

curialis. Mg.

scutellata. Fll.

plumulifera. Lw.

signata. Eg*g.

means. F.

Hercyniae. Lw.

pulchripes. Lw.

means. Ztt.

urbanus. Mg.

paganus. Mg.

carbonaria. Egg.

cynocephala. Lw.

impressa. Lw.

vernalis. Mg.

coemeterionum. Fll.

rulpina. $\mathrm{Mg}$.

pallidicornis. $\mathbf{M g}$.

proxima. Ztt. albitarsis. Mg.

flavimana. Ztt. Mg. vidua. Meq.

morio. Ztt.

lineata. Whlbg.

luteicornis. Ztt.

mutabilis. Fll.

pyomaea. Ztt.

funeralis. $\mathrm{Mg}$.

ruralis. Mg.

pratensis. IIg.

fasciata. Schin. u. Egg.

canicularis. $\mathrm{Pz}$.

aurata. Rond. F.

montana. Egg.

himantopus. Ztt.

fraterna. $\mathbf{M g}$.

pictipennis, Fgg.

flavicornis. F.

flavipes. Fll. pt. Pz.

gilvipes. Ztt.

flavipes. Fll. pt.

fulvipes. $\mathrm{Ltt}$.

chrysocoma. Mg.

grossa. Fll.

phantoma. Ztt.

c̀bloris. Mg.

brachysoma. Egg.

modesta. Egg.

vernalis. Fll.

abscur $\alpha$. Lw.

praecox. Ztt.

auripila. $\mathrm{Mg}$.

albipila. $\mathrm{Mg}$.

luctrosa. Mg.

velutina. Lw.

griseiventris. Lw.

dimidiata. Ztt.

gigantea. Ztt.

olivacea. Ztt.

alpina. Ztt.

rufitarsis. Ztt.

inmupta. Ztt. glírina. Rond.

testacicornis. Rond.

pusilla. Rond.

nigricornis. Meq.

nitida. $\mathrm{Mg}$.

limbata. Heq.

Schineri. Egg:

rufitibia. Egg.

fulvicornis. Mg.

means. $\mathrm{Mg}$.

viduata. F.

vidua. $\mathrm{Mg}$.

chalybeata. Mg.

albiseta. Mg.

placida. $\mathrm{Mg}$.

geniculata. $\nabla$. Ros.

geniculata. Ztt.

latifrons. Ztt.

laticornis. Roud.

longula. Ztt.

lugubris. Ztt.

rufipes. Mcq.

pedemontana. Roud.

subalpina. Rond.

tarsata. Mcq.

fulvipes. Mg.

aerea. L. Duf.

ahenea. y. Ros.

linearis. $\mathrm{r}$. Ros.

atra. Gimmerth.

523. Leucozona. Schin.

- Musca. L. - Syr-

phus. F. Mg. - Eri-

stalis. F. - Cheilosia.

Wlk. - Conops. Scop.

lucorum. L.

praecincta. Scop.

asiliformis. F.

624. Eriozona. Förster.

- Syrphus. Mg. -

Scavva. Fll, Ztt. 
syrphoides. 111.

oestriformis. Mg.

Folucellinae.

625. Brachyopa. Mg. - Musca. Pz. Rhingia. Fll. ferruginea. Fill. conica. P'z.

testacea. Fll, pt. dorsata. Ztt. arcuata Pr. bicolor. Fll. cinerea. Whlbg: testacea. Fll. vittata. Ztt. scutellaris. R.-Dest. 626. Rhingia. Scop. Conops. I. - Volucella. Geoffr.

rostrata. I.

campestris. Mg. rostrata. Deg. austriaca. $\mathrm{Mg}$.

627. Volucella. Geoffr. - Musca. L. - Conops. Scop. - Synphus. F. Fll.

bombylans. L. yocopyges. Poda. tricolor. Poda. plumata. Deg: mystacea. Pz. L. pennata. Scop. proxima. Rond. apiaria. Schrk. haemorhoidalis. Ztt. pellucens. $L$. dryaphila. Scop. inflata. F. dryaphila. Schrk. Hockhrthi. Gimm. dryophila. Rond. zonaria. Poda. inanis. F. fasciata. Herr.-Schff. bifasciata. Scop. inanis. $\mathbf{L}$.

apivora. Deg. trifasciata. Scop. micans. F. liquida. Erichs. analis. Meq. vulpina. Mg. elegans. Lw.

Sericomyinae. 628. Sericomyia. Mg. - Musca. L. - Syrphus. F. Fll. borealis. Fll.

laxponum. Ltr. pt. lappona. Deg. pt. lappona. L.

629. Arctophila. Schin. - Musca. Gmel. Syrphus. F. Fll. Sericomyia. Mg. pt. bombyformis. Fll. mussitans. F.

superbiens. Miller.

Eristalinae.

630. Eristalis. Ltr. Musca. L. - Conops. Scop. - Syrphus. Ztt. - Elophilus.Ltr. sepulcralis. L. pt. tristis. F. aeneus. Scop. stygius. Newm. flavicornis. Rossi. apiformis. Ell. cryptarum. F. nubilipennis. Curtis. tenax. L. vulgaris. Scop. fuscus. Scop. campestris. $\mathrm{Mg}$. hortorum. $\mathrm{Mg}$. porcinus. Deg. arbustorum. Schrk. vulpinus. $\mathrm{Mg}$. intricarius. L.

leucorhoeus. Scop. aureus. $\mathrm{Pz}$. bombyliformis, $\mathrm{Pz}$. arbustorum. L. jugorum. Egg. rupium. F.

piceus. FIl. fossarum. MIg. pratorum. $\mathrm{Mg}$. nigroantennatus. Schumm. pascrorum. Rond. pertinax. Scop. similis. Fll. sylvarum. Mg. nemorum. L. MIg. lucorum. Mg. alpinus. $\mathrm{Pz}$. horticola. Deg. flavocinctus. F. fraterculus. Ztt. anthophorinus. Fll. nitidiventris. Ztt. taeniops. $W^{T}$. pulchriceps. $\mathrm{Mg}$. fasciatus. $\mathbf{L w}$. quinquelineatus. E. fasciatus. Mg. ridens. WVIk. saltuum. Rond. 
nigrifrontis. Brullé. nigritarsis. Meq. taphicus. W.

631. Platynochoetus. IV. - Surphus. F. - Eristalis. E.

setosus. F.

Macquarti. Lw.

632. Helophilus. Mg. - Musca. L. - Conops. Scop. - Syrphrs. Pz. Fll. - Eirstalis. F. $\mathrm{MIg}$. - Elophilus. Ltr. - Rhingia. F. pt. - Myathropa. Rond. pt.

Myathrop a. Rond.

floreus. 1 .

atropos. Schrk.

nigrotarsatus. Schin.

Hclophilus.

hybridus. Lw.

trivittatus. Mg. trivittatus. F. pendulus. L. peregrinus. Lw。 frutetorum. F.

femoralis. Fll. versicolor. $\mathrm{F}$. lumulatus. Mg. groenlandicus. F.

arcticus. Ztt. pt. lapponicus. Whllog.

areticus. Ztt. pt. affinis. Whlbg.

arcticus. Ztt. pt. bottnicus. Whibg. borealis. Staeg. solitarius. Rond.
Lejops. Rond.

vittatus. Mg.

Ruddii. Curtis.

A $x a \operatorname{sim} y i a$.

transfugus. L.

lineatus. F.

muscarius. Pz.

femoratus. Pz.

camporum. JIg.

633. Mallota. Mg. Syrphus. F. Fll.

Eristalis. F. Itr. -

Zetterstedtia. Rond.

- Imatisma. Mcq. pt.

Mallote.

fuciformis. $\mathrm{F}$.

megilliformis. Fll.

cristalloides. I w w.

cimbiciformis. Ztt.

$I m$ atis $m x$.

posticata. F.

cimbicijormis. Fll.

634. ITerodon. Iig. Syrphus. F. - Milesia. Ltr. F. pt. equestris. F.

fuciformis. Schellenb. nobilis. Mg.

transversalis. Mig. constans. Mig. nrreissi. F.

ferrugineus. F.

tlavicans. F.

tuberculatus. Rond.

bulborum. Rond. validus. $\mathrm{Mg}$. claripes. F.

gravipes. Rossí. curvipes. Gmel.

filvus. Mleq. sicanus. Rond. aber'ans. Egg'. avidus. Rossi.

pruni. Rossi.

rufitibius. Rond. nigritarsis. Rond. spinipes. F. viaticus. $\mathrm{F}$. armipes. Rond. albifrons. Mg. ruficornis. Mg. cinereus. $\mathbf{F}$.

posticatus. $\mathrm{Mg}$. analis. Mg: funestus Rond. osmioides. Perris. rufus. $\mathrm{Mg}$. aeneus. $\mathbf{M g}$. aureus. F. annulatus. $\mathrm{F}$. melancholicus. $\mathrm{F}$. natans. $\mathrm{F}$. italicus. Rond. graecus. WVlk. senilis. Mg. varius, Rond. serrulatus. Mg: parietum. Mg: moenium. $\mathrm{Mg}$. montanus. Rond. subfasciatus. Rond. pudicus. Rond. submetallicus. Rond. aerarius, Rond. mucronatus. Rond. chalybeus. $M g^{*}$. inermis. Meq. graecus. I,w. 
anrifer. Lw.

planiceps. Lw.

635. Tropidia. $\mathrm{Mg}$.

Eristalis. Fll.

fasciata. Mg:

milesiformis. Fll.

rufomaculata. Curt.

dorsalis. Meq.

Marsanii. Perris.

\section{Milesina $\theta$.}

636. Milesia. Ltr Syrphus. F. Rossi. Eristalis. F. - Splizaea. Rond.

crabroniformis. F. givas. Rossi. splendida. Rossi. fulminan: $\mathrm{F}$.

637. S pilomy ia. Mig.Musca. L - Syrphus. F. - Eristalis. F. Fll. - Milesia. Auct. pt. - Temnostoma. St. Farg. und Serv. - Caliprobola. liond. pt.

reminostoma.

saltuum. F. diophthalma. Mg. diophthalma. L. saltuum. Nig. respiformis. L. apiformis. P'z. Wague. Gorski. apiformis. F. Ztt. bombylans. F.

Zetterstedtii. F11. Caliprobola. speciosa. Kossi.
63.38. Xylota. MIg. - 6.10. Brachypalpus. Musca. L. - Syrplus. F. Pz. - Nilesia. Ltr. - Helophilus. Mg. ol. Eumeros. Mg. ol. Micraptoma. Westw. segnis. L.

triangularis. Ztt. pt. confinis. Ztt.

pigra. F.

crassipes. Whlbg.

lenta. $\mathrm{Mg}$.

pigra. Pz. Fll.

ignava. Pz.

femorata. L. volvulus. F. Mg. curvipes. Lw. nemorum. $\mathrm{F}$. ignava. Fll. floon. Zatt. lifarciata. Mg. nigripes. Ztt.

florum. F. $\mathrm{Mg}$. nemorum. Zitt. abiens. Mg. caeruleiventris. Ztt. triangularis. Ztt. sylvarum. L. impiger. $\mathrm{Pz}$. fulvirentris. Bigot.

tarda. Mg. 639. Syritta. St. Farg. u. Serv. - Musca. L. - Conops. Scop. - Syrphus. Pz. Fll.

- Milesia. Ltr. F. fir. Plocota. St. Farg. - Xylota. Mg. Mestw. - Coprina. Ztt. ol.

pipiens. 1. Plocota. St. Farg.
u. Serr. - Musce.
Schrk. - Milesia.
Mg. Criothina. Mg.
- Dasymyir. Egg. Plocota. St. Farg.
u. Serr. - Musce.
Schrk. - Milesia.
Mg. Criothina. Mg.
- Dasymyir. Egg. Plocota. St. Farg.
u. Serr. - Musce.
Schrk. - Milesia.
Mg. Criothina. Mg.
- Dasymyir. Egg. Plocota. St. Farg.
u. Serr. - Musce.
Schrk. - Milesia.
Mg. Criothina. Mg.
- Dasymyir. Egg.

Mcq. - Syophus. l'z. Fll. - Milesia. F. Meradon. F. - Xylota. $\mathrm{Hg} . \mathrm{Ztt} . \quad-$ Criorhina. MIg. angustus. Egg. Meigenii. Schin. femoratus. Mg: valgus. I'z. lapleriiformis. FlI. olivacers. Micq. tuberculatus. Heq. bimaculatus. Mcq. chrysites. Egg". refipilus. Egg: ol. 641. Criorhina. Meq. Musca. Deg. - Eristalis. F. Ltr. - Syspius. Fll. - Milesia u. Aylota. Mlg. ol. pachymera. Eggg. ruficauda. Deg. haemorhoidalis. Alg. ranunculi. $\mathrm{Pz}$. fallax. L. seminufa. $\mathrm{F}$. berberina. $\mathrm{F}$. asilica. Fll. rufipila. $\mathrm{IIg}$. oxyacanthae. Hy. floccosa. Mg. regula. Fll. Brebissonii. Mcq. flavicauda. Meq. bombiformis. Perris. apiformis. Meq. spinigera. Jw. 
apiformis. Schrk.

apicata. $\mathrm{Mg}$.

643. Myolepta. Newm. - Mrusca. Gmel. Thereva. F. - Eristalis. Fll. - Xylota. Mg. pt. - Xyloteja. Rond. - Criorhina. Mg. - Brachypalpus. Meq. pt.

Iuteola. Gmel.

dubia. F.

lateralis. Fll. ruficornis. Ztt. vara. $\mathrm{Pz}$.

644. Eumerus. Mg. Syiphus. F. Pz. Eristalis. F. - Milesia. Ltr.

oratus. Isw.

tricolor. Lw. ol.

licolor. Rond.

annulatus. $\mathrm{Pz}$.

varius. $\mathrm{Ig}$.

lateralis. Ztt.

grandis. $\mathrm{Mg}$. tarsalis. Lw. sinuatus. Lw. tricolor F. mixtus. Pz. Mg. sabulonum. Fil. strigatus. Mg. Selene. Mg. rubriventris. MIeq. longicornis. Lw. elegans. Schin. Egg. cilitarsis. Lw. ornatus, $\mathrm{Mg}$. rnficornis. Mg.

barbiventris. Rond. lunulatus. MIg. planifrons. Mg. funeralis. Mg. grandicornis. $\mathrm{MIg}$. strigatus. Fll. aeneus. Mcq. Selene. Lw. ol. litoralis. Curtis.

latevalis. Schin. micans. F. barbarus. Coqueb. australis. $\mathrm{Mg}$. Hlavitarsis. Ztt. fulvicornis. Meq. ruficomis. Meq. ol. immarginatus. Mcr. olivaceus. Lw. nudus. Lw.

Iris. L,w. emarginatus. Lw. cavitibius. Rond. clavitibius. Rond. basalis. Lw, angusticornis. Rond. pulchellus. Lw. delicatae. Rond. amoenus. Lw. pusillus. Lw. argyropus. Lw. exilipes. Rond. uncipes. Rond. Truquii. Rond. tuberculatus. Rond. lasiops. Rond. lejops. Rond. melanopus. Rond. alpinus. Rond.

645. Chrysochlamys. Rond. - Conops. Scop. - Syrphrs. F. Pz. - Elophilus. Ltr. - Eristalis.Fll Ztt.Cheilosia, Mcq.Mg. Ferdinandea. Rind. ol. cuprea. Scop.

ruficonnis. Pz. Mg. ruficornis. F. aurea. Rond.

Chrusotoxinae.

646. Or thoneura. Mcq. - Eristalis. Fll. -Chrysogaster. Mg. Camponeura. Rond. pt.

elegans. Mg. genieulata. Mg. fuscipennis. Ztt. ol. nobilis. Fll.

nigricollis. Mg. brericornis. Lw. plumbago. Lw. frontalis. Lw. venusta. Rond.

647. Chrysogaster. Mg. - Musca. L. Syrphus, F. Pz. Eristalis. Fll. splendidus, Mg. tarsatus, Mg. rufitarsis. Lw. coenotaphii. $\mathrm{Mg}$. metallinus. F. metallicus. $\mathrm{F}$. dissicornis. $\mathrm{Mg}$. grandicornis. Mg. riduatus. L. mudus. Meq. Macquarti. Lw. aerosus. $\mathrm{Lw}$. aeneus. Mg. splendens. Mg. violaceus. $\mathrm{Mg}$. coemeteriorum. I. solstitialis. Ztt. chalybeatus. Mg. 
virescens. Lw.

basalis. Lw.

inornatus. Lw.

simplex. Lw.

incisus, $L w$.

hirtellus. Lw.

nubilis. Rond.

bicolor. Meq.

caerulescens. Mcq.

amethystinus. Mcq.

fumipennis. Steph.

insignis. Lw.

longicomis. Lw.

648. Psilota. Mg. -

Pipiza. Fll.

anthracina. $\mathrm{Mg}$.

conjugata. Rond.

inupta. Rond.

nigra. Ztt

atra. Fll.

649. Triglyphus. Lw. primus. Lw.

650. Pipiza. Fll. - Musca. L. - Syrphus. Pz. - Eristalis. F. - Mulio. F. - Milesia. Ltr.

fasciata. Mg. quadrimaculata. Pz. festiva. $\mathrm{Mg}$. ornata. $\mathrm{Mg}$. lunata. Mg. artemis. $\mathrm{Mg}$. noctiluca. L. rosarum. Pz. luteitarsis. Ztt. signata. Mg. geniculata. Mg. bimaculata. Mg. notata. Mg.

flavitarsis. $\mathrm{Mg}$. vitrea. $\mathrm{Mg}$. Ratzeburgi. Ztt. guttata. Mg. fenestrata. Mg. lugubris. F. moesta. Gmel. austriaca. Mg. funebris. Mg. lugubris. Mg. vitripennis. Mg. chalybeata. Mg. caerulescens. Micq. anthracina. $\mathrm{Mg}$.

? ruficornis. Mg. acuminata. Lw. carbonaria. $\mathrm{Mg}$. leucozoncs. Ztt. geniculata. Zitt. leucopeza. Mg. calceata. $\mathrm{Mg}$. obscuripennis. $\mathrm{Mg}$. lucida. Mg. binotata. Ztt. biguttula. Ztt. pt. hyalipennis. Ztt. obscura. Meq. obsoleta. Zitt. quadriguttata. Meq. fulvimana. Ztt. morionella. Ztt. vana. Ztt. nigripes. Mcq. fulvitarsis. Mcq. albipila. $\mathrm{Mg}$. albitarsis. Mg. luctuosa. Meq. rufithorax. Mg. tristis. Mg. stigmatica. Ztt.
65้1. Pipizella. Rond. - Mfulio. F. - Pipiza. Fil. Mllg. Heringia. Rond. pt. virens. $\mathrm{F}$.

campestris. Fll. maculipennis. Mg. geniculata. Curtis. varians. Rond. annulata. Meq. morosa. Lw. melancholica. $\mathbf{M g}$. interrupta. Hal.

Heringia. Rond.

Heringi. Ztt. Zetterstedti Rond.

652. Paragus. Ltr. Mulio. F. - Syrphus. Pz. - Pipiza. Fll.

tibialis. Fll.

tarsatus. Rond. haemorrhous. Mg. trianguliferus. Ztt. sigillatus. Curtis. aeneus. $\mathrm{Mg}$. obscurus. Mg. femoratus. $\mathrm{Hg}$. disya.: Schumm. niyritis. Gimmerth. albipes. Gimmerth. coadunatus. Rond. cinctus. Schin. u. Egg. albifrons. FIl.

thymiastri. Ltr. ater. $\mathrm{Mg}$.

majoranae. Rond. quadrifasciatus. $\mathrm{Mg}$.

bifasciatus. Mcq. productus. Schin. lacerus. $\mathrm{I} w \mathrm{w}$. 
bimaculatus. IV. strigatus. $\mathrm{Mg}$. bicolor. F. metannclirysa. Gmel. zonatus. Mg. testacers. $\mathrm{Mg}$. arcuatus. IIg. taeniatus. $\mathrm{Mg}$. prenctulatus. Ztt. ruficauda. Ztt.

Pecchiolii. Rond. ebracteatus. Rond. sexarcuatus. Bigot.

633. Chrys ot ox um. MIg. - Musea I. - Conops. Scop. - Mulio. F. Fll. - Syrphus. Pz. - Milesia. E.

fasciolatum. Deg. marginatum. $\mathrm{Mg}$. costale. $\mathrm{Mg}$. arcuatum. L. fasciolatum. Fll. hortense. Mg. sylvarum. Mg. fasciolatum. $\mathrm{Mg}$. arcuatum. Wlk. scutellatum. Hcq. vespiforme. $\mathrm{F}$. cisalpinum. Rond. intermedium. Mg. scutellatum. Lw, ol. monticola. Schumm. graecum. WIk. italicum. Rond. bicinctum. L. tricinctum. Rond. lineare. 7 tt. festivum. L.

arcuatum. Fll. Mg. veruale. Lw. octomaculatum. Curtis. devius. L. intermedium. Ztt. arcuatum. Fll. pt. chrysopolita. Rond. elegans. Lw.

intermedium. Wlk. arcuatum. Schrk. parmense. Rond. impudicum. Lw. collinum. Rond. lunulatum. Brullé. 60'́ Psarus. Ltr.

Syrphus. F. abdominalis. F.

655. Sp h e com yia. Ltr. - Chrysotorum. W. - Psarus. IV. Tyzenhausia. Gorski. respiformis. Gorski.

630. Callicera. Pz. Bibio. F. - Syrphus. Rossi.

aenea. F.

aurata. Rossi.

rufa. Schumm.

Spinolae. Rond.

Roserii. Rond.

Nacquartii. Rond.

Porrii. Rond.

Bertolonii. Rond.

Fagresii. Guér.

Microdoninae.

657. M icrodon. Mg. Musca. L. - Mulio. F. - Stratiomys. Pz. - Aphritis. Ltr. mutabilis. I. apiformis. Deg. apiarize. F. nours. Schrk. auropubescens. Ltr. scutellatus. Schumm. piger. Schrk.

micans. Mg.

anthinus. $\mathrm{Mg}$.

apiformis. Curtis.

conicus. Pz.

plebejus. Schrk.

latifrons. Lw.

brevicornis. Egg.

Cerinae.

658. Ceria. F. - Musea.

L. - Conops. Schurk.

- Syrplues. Pz. -

Sphiximorpha. Rond. pt.

conopsoides. L. conopsea. Pz. clavicornis. $\mathbf{F}$. vaginicornis. Schrk. subsessilis. Ltr. clavicomis. MIg. ol. vespiformis. Ltr. scutellata. Mcq.. intricata. Saund. Petronilla. Rond. Garibaldii. Rond.

XXX. Conopidae. Myopinae.

6.59. Dalmannia. R.Dest. - Myopa. F. Mg. - Stomoxys. F. - Stachynia. Meq.

- Conops. L.

aculeata. L.

australis. Mcq. meridionalis. R.-Dsv. gemina. Mg. desponsata. Rond. punctata. F. virens. Fll. 
Harescens. Mg.

marginata. $\mathrm{Mg}$. marginella. Ztt. parvula. Rond. flavipes. R.-Dest.

dorsata. Mg. dorsalis. F. scutellaris. Enc. méth. 662. Glossigo on a. Rond. flavipes. Meq vernalis. R.-Desv. 660. Муора. F,- Conops. L. - Phorosia, Myopella, Purpurella, Haustellia, Myopina, Fairmairia, Lonchopalpus, Sicus u. Pictina. R.Desr. - Gonis/lynchus. Rond, pt. stigma. Mg. scutellaris. R.-Desv. variegata. $\mathrm{Mg}$. nitidula. F. picta. Pz.

buccata. L. fulvipalpis. R.-Desv. puncticeps. R.-Desv. florea. R. - Desv. maryinalis. R.-Desv. punctigera. R.-Dest. testacea. L.

buccata. Gmel. Scop. ferruginea. $\mathrm{Pz}$. polystigma. Rond. pictipennis. R.-Desv. occulta. Mg. dispar. Rond. fasciata. $\mathrm{Mg}$. ephippium. E. dorsalis. F.

testacea. F. grandis. $\mathbf{M g}$. nobilis. R.-Desv. morio. II 0 .

puella. Rond.

maculata. Meq.

annulata. F. Auct.

ferrugineus. L.

-- Myopa. Auct. Melanosoma. R.Desv.

bicolor. $\mathrm{Mg}$.

nigra. Mg.

nupta. Rond.

distincta. R.-Desv. nigripes. R.-Desr. brunnipes. R.-Dest. Zetterstertii. R.-Desv. pallipes. Mg.

663. Occemyia. R.Desv. - Myopa. distincta. $\mathrm{Mg}$. atra. F.

cinerascens. $\mathrm{Mg}$. maculata. Mg. micans. Mg. femorata. F. femoralis. R.-Desv. nitidula. R.-Desv. fulvifrons. R.-Desv. Meigenii. R.-Desv. pallipes. R.-Desv. Dufouri. R.-Dest. grisea. R.-Desv.

Lamarckii. R.-Desr. Macquarti. R.-Desr. Guerini. R.-Desv. Bigoti. R.-Desv. brunnipes. R.-Desv. Lucasi. R.-Desv. pusilla. R.-Desv. nana. R.-Desv.
661. Sicus. Scop. - Conops. L. - Myopa. Auct, - Thecophora. 665. Conops. L. - Bra-
Sunderalli. Ztt. atra. Fll. pt. pusilla. Mg. nana. Meq. fulvipes. Mcq. melanopa. Rond. 664. Zodion. Ltr. Муори. Е. Fl. cinereum. F. tibiale. F. conopsoides. Ltr. fuliginosum. R.-Dsv. fulvipes. R.-Desv. fulvicorne. R.-Desv. Carcelii. R.-Desv. pediciliatum, R.-Dsv. notatum. $\mathrm{Mg}$. irroiatum. F. tessellatum. F. frontale. F.

Conopinae. chyglossum, Leopoldius, Conopoides, Conopaejus, Conopilla u. Sphixosoma. Rond. signatus. $M g$.

brevirostris. Germar. erostratus. Rond.

capitatus. Lw. coronatus. Rond.

diadematus. L.w. diadematus. Rond. scutellatus, Mg*. silaceus. Mg. vesicularis. I.

cylindricus. $\mathrm{MIg}$. ol. claviconis. Fourcroy. macrocephalus. L. quadrifasciatus. Deg. aculeatus. F. vitellinus. I,w. mueronatus. Rond. 
insignis. Lw.

flavifrons. Mg. ceriaeformis. $\mathbf{M g}$. acuticornis. $\mathrm{Lw}$. strigatus. Mg.

trifasciatus. $\mathrm{Mg}$. auricinetus. Lw.

flavipes. L.

trifasciatus. Deg. melanocephalus. $\mathrm{Mg}$. bicinctus. Mg. ferrugineus. MIcq. calceatus. Rond. aureifasciatus. Rond. terminatus. Mcq. elegans. $\mathrm{Mg}$. maculatus. Heq.

666. Ph y s o e p hala. Schin. - Conops. Auctor.

nigra. Deg. macrocephala. F. rufipes. F. lacera. Mg.

annulipes. $\mathrm{Mg}$. pusilla. Mg.

tener. Lw. truncata. Lw. chrysorrhoea. Mg. Pallasii. Mg. variegata. Mg. vittata. F.

chrysorrhoea. Zell. soleaformis. Gimm. semiatra. Costa. fraterna. Lw, dorsalis. Mg. meridionalis. Meq. laticincta. Brullé. pumila. Mcq.

\section{B. Eproboscidea.}

XXXI. Hi poboscidae.

Hip poboscinae.

667. Melophagus. Ltr. - Hippobosca. Ltr. F. $\mathrm{Pz}$.

ovinus. L.

668. Lip op ten a.Nitzsch.

- Pediculus. L. Melophagus u. Ornithobia. Mg. - Leptotena. Meq. - Haemobora. Curtis. - Alcephagus. Gimmerth. cervi. L.

pallida. Mg. pallipes. Curtis.

669. Ornith o m y ia. Ltr. - Hippobosca. Auct. avicularia. L. viridis. Leach. vividula. Mg. fringillina. Curtis. metallica. Schin. tenella. Rogenh. ptenoletis. Lw. turdi. Ltr.

6\%. Leptopteryx. Ztt. nivalis. Ztt.

671. St e 11 o ptery $\mathrm{x}$. Leach. - Hippobosca. Auct. - Ornithomyia. Ltr. - Craterina. Curtis.

hirundinis. L.

672. Oxy terum. Leach. - Hippobosca. Pz. - Anapera. $\mathrm{Mg}$. pallidum. Leach.

hivundinis. $\mathrm{Pz}$.

Kirbyanum. Leach. sibiricum. Gimmerth.

673. Olfersia. W. -

Feronia. Leach.

ardeae. Mcq.

metallica. Schumm.

Courtilleri. Fairm.

674. Hippobosca. L.

- Nirmomyia. Nitzsch.

equina. L.

uralensis. Enc, méth. camelina. Leach.

Francillioni. Leach.

Braulinae.

675. Braula. Nitzsch. Entomobia. Costa. caeca. Nitzsch. apum. Costa.

XXXII. Nycteribidat. 676. Nycteribia. Ltr. - Acarus. L. Hippobosca. Voigt. - Phthirium. Herm.

- Celeripes. Montaga.

Listropodia. Kolen.

Blasii. Kol.

Schmidlii. Schin.

Latreillii. Leach. vespertilionis. Ltr. pedicularia. Westw. Nattereri. Kol.

Acrocholidia. Kolen.

vexata. Westw.

Montagui. Kolen. vespertilionis. Herm. Bechsteinii. Kolen. 
Sty lidict kiolen.

Hermanni. Leach.

biarticulata. Westw.

Herm.

vespertitionis. Mon- XXXIII. Louchopterida e. tagui.

Penicilidia. Kolen.

Westwoodii, Kol.

Dufouri. Westw. vespertitionis. Duf. Frauenfeldi. Kol.

Leachii. Kol. yespertilionis. L.

pedicularia. Ltr.

Incertae sedis.

677. Louchoptera. Mg. - Dipsa. Fll. lutea. $\mathrm{Pz}$. furcata. $\mathrm{Fll}$. rivalis. Mg. trilineata. Ztt. punctum. Mg. flavicauda. $M \mathrm{~g}$. lacustris. $\mathbf{M g}$. tristis. $\mathrm{Mg}$. riparia. Mg. thoracica. Mg. impicta. Ztt. cinerella. Ztt. fuscipennis. Boh. nigrimana. Mg. palustris. Mg: cinculata. Gimm. grisea. Gimmerth. 






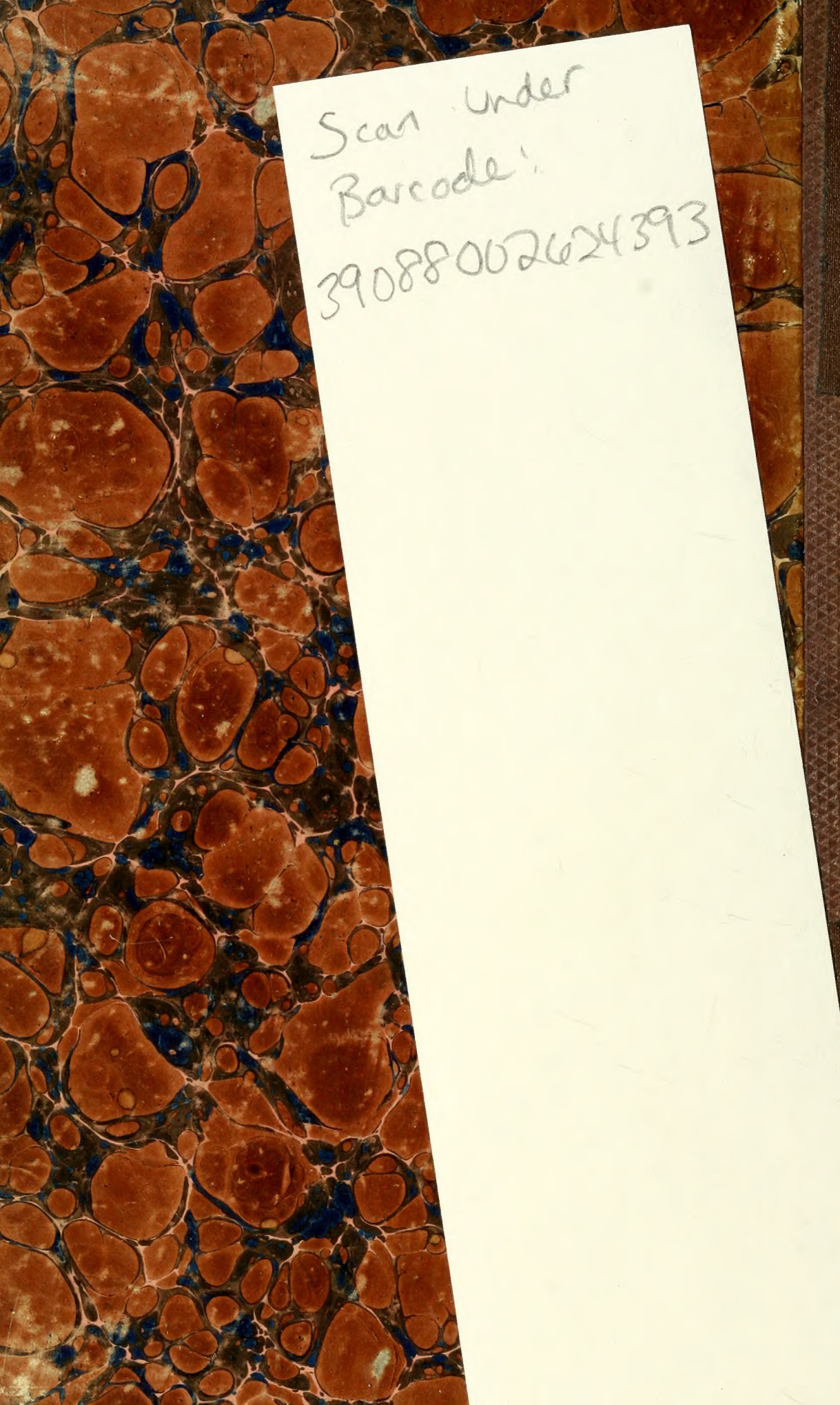

